\title{
ABUNDANCES OF THE HEAVY ELEMENTS IN THE MAGELLANIC CLOUDS
}

\author{
Stephen Charles Russell
}

\begin{abstract}
A thesis submitted for the degree of Doctor of Philosophy
of The Australian National University
\end{abstract}

June 1989

Mount Stromlo and Siding Spring Observatories

The Australian National University

Canberra 
In memory of my father 
This thesis consists of a series of three papers that have been or will be submitted to the Astrophysical Journal. The work described herein is the work of the candidate alone, except where described below or acknowledged in the text.

Paper 1 The observations were carried out by the candidate with the help of Dr. M. S. Bessell, Dr. J. Norris, Dr. M. Ashley, R. J. V. Brissenden, S. Wayte, G. Meurer, M. Winsall, and T. Bruce. The analysis, and the discussion is the work of the candidate alone, but with many helpful comments and suggestions from Dr. M. S. Bessell.

Paper 2 The observations were carried out by the candidate with the help of Dr. M. A. Dopita. The analysis was carried out by the candidate alone, and the discussion is mainly the work of the candidate but with some important insights derived from Dr. M. A. Dopita.

Paper 3 The modelling program discussed in this paper was developed by Dr. M. A. Dopita alone. Both the candidate and Dr. M. A. Dopita contributed equally to the discussion of the modelling program. The modelling, itself, and the description of the results is due to the candidate alone. Equally,the description of the abundance results is due to the candidate alone. Both the candidate and Dr. M. A. Dopita contributed equally to the discussion and conclusions.

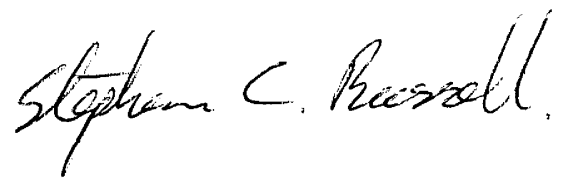

Stephen C. Russell 


\section{ACKNOWLEDGEMENTS}

I would like to express my gratitude, first and foremost, to my principle supervisors Michael Dopita, and Michael Bessell. Their patience, support and encouragement were invaluable. Thanks also to Drs. P. R. Wood and G. H. Smith for their helpful comments and discussions, and to all the staff and fellow students at this observatory for their friendship and support.

Thanks especially to the former director of the Mount Stromlo and Siding Spring Observatories (MSSSO) Donald Mathewson, and the present director Alex Rodgers, for their constant support, particularly at times of my great personal distress due to the untimely loss of family members.

I would like also to thank the Time Assignment Committee of MSSSO, and the Australian Time Allocation Committee of the Anglo Australian Observatory, for granting me the telescope time necessary for completing my program of observations.

I am grateful to the financial support I received from an Australian Commonwealth Postgraduate Research Award for the term of this thesis.

I am indebted to the support, encouragement and good spirits of my wife Anita. These long years of study would not have been a tenth as much fun without her. Thanks also to my children Damien (now deceased),Kevin, andSonja, who have provided a great source of entertainment, especially during all those nights when I had nothing better to do than sleep. A special thanks to my father, Dr. John Russell, who helped so much with fostering the love I now have of the quest for knowledge. Thanks to my mother Marjorie, for her devotion and financial support, especially in these final months. 
'Just the place for a Snark!' the Bellman cried, As he landed his crew with care; Supporting each man on the top of the tide By a finger entwined in his hair.

'Just the place for a Snark! I have said it twice:

That alone should encourage the crew. Just the place for a Snark! I have said it thrice: What I tell you three times is true.' 


\section{ABSTRACT}

This thesis presents the first consistent detailed abundance analysis of the range of elements available for study in the Large and the Small Magellanic Clouds (LMC and SMC respectively). Reliable abundances have been obtained for $\mathrm{C}, \mathrm{Mg}$, $\mathrm{Ca}$ and heavier elements, from fine analysis of high dispersion spectra of 8 lower luminosity F-supergiants in each of the Clouds. The lighter elemental abundances, in particular for $\mathrm{He}, \mathrm{O}, \mathrm{N}, \mathrm{Ne}, \mathrm{S}$ and $\mathrm{Ar}$, have been obtained by modelling the emission spectra of a sample of bright, compact $H \Pi$ regions in both of the Clouds. Overlap between the two, otherwise disparate abundance scales, has been obtained by modelling the emission spectra of supernova remnants in each Cloud. These provide the abundances of the same elements observed in $\mathrm{H}$ II regions, as well as the metals $\mathrm{Ca}, \mathrm{Cr}, \mathrm{Fe}$ and $\mathrm{Ni}$. The latter four elements were measured differentially with respect to a sample of Galactic Herbig-Haro objects which are assumed to be of solar composition.

The observations provide the first high dispersion spectra of a group of $F$ supergiants in both Clouds that are of sufficiently low luminosity for their physical parameters to be determined with a high degree of confidence. Observations of the $\mathrm{H} I I$ regions include spectra taken in the near-infrared wavelength region from $7500 \AA$ to beyond $1 \mu \mathrm{m}$. This extends the optical spectra, quoted in the literature for these objects, to include several potentially valuable emission lines. The supernova remnants (SNRs) observed included the hitherto unobserved object SNR 0104-723 in the SMC, bringing the number of SNRs with measured spectra in that galaxy to a grand total of two. The spectra of the SNRs were also extended to beyond $1 \mu \mathrm{m}$, hence including a valuable array of heavy metal lines otherwise unattainable.

It is shown that the Fe abundances in the SMC and LMC are respectively -0.65 dex and -0.30 dex relative to the Sun. In addition, it is shown that there is no detectable zero point error between the abundance scales derived from the supergiants and the $\mathrm{H}$ II regions. Carbon abundances, traditionally thought to be 
severely depleted in the Clouds from UV observations of $\mathrm{H} I I$ regions, are shown to be undepleted relative to $\mathrm{Fe}$ in the atmospheres of F-supergiants. This is believed to be a true indication of the $\mathrm{C}$ abundance of the interstellar medium (ISM) in the Clouds, while the $\mathrm{H} I I$ regions are believed to be depleted in $\mathrm{C}$ due, perhaps, to the presence of graphite dust grains.

The overall abundance patterns of the Clouds show two particular deviations from strict proportionality with Fe relative to the Sun. The first is a dropping off in the relative abundances of the lighter elements with decreasing atomic number, which is however, seen to disappear when the abundances are plotted relative to the local Galactic ISM. This is interpreted to indicate that the present local ISM is different to the ISM that produced the Sun, the latter having long since fallen further in towards the Galactic center. The second deviation from proportionality with iron occurs for the very heaviest elements. These are shown to have a distribution in abundances beyond $\mathrm{Zr}(\mathrm{Z}=40)$ that is little different from that of the Galactic halo, which is in turn, thought to be a result of r-processing only, with no contribution from the s-process. This is interpreted to indicate that the efficiency of the intermediate mass stars in producing the heavier s-process elements is severely reduced for the elements heavier than $\mathrm{Zr}$ in the SMC, and heavier than $\mathrm{Ba}$ in the LMC. Conversely, the high mass stars are interpreted as being more efficient producers of the r-process elements in the Clouds than in the Galaxy since the initial mass function (IMF) is steeper in the Clouds.

The chemical and physical properties of the Magellanic Clouds were successfully modelled assuming bimodal star formation and gas infall. The end points for the models were constrained by the ratio of the gas mass to the total mass, the age of the system, the peak in the star formation rate, and the oxygen to iron ratio. The models indicate that the star formation peak in a galaxy broadens, and is further delayed in time, as the galaxy mass decreases. There is also some support for the conjecture that star formation efficiency is relatively constant for galaxies above some critical mass, but the efficiency falls off below this mass. 


\section{TABLE OF CONTENTS}

ABSTRACT

TABLE OF CONTENTS …........................................... viii

INTRODUCTION

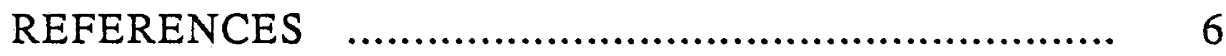

Abundances of the Heavy Elements in the Magellanic Clouds.

I. Metal Abundances of F-type Supergiants ....................... 8

ABSTRACT

I. INTRODUCTION …............................................. 10

II. PHOTOMETRY …........................................... 18

a) The Observations .............................................. 18

b) Reddenings .................................................... 23

c) Photometric Derivation of the Stellar Physical Parameters .. 27

III. SPECTROSCOPY ............................................... 32

a) The Observations …............................................. 32

b) The Analysis Technique ...................................... 41

c) The Absolute Abundance Analysis of Canopus ............. 60

d) The Differential Analysis ...................................... 62

e) Estimation of the Physical Parameters for the Program Stars 62

f) The Accuracy in the Determination of the Abundances ..... 71

IV. ELEMENTAL ABUNDANCES .............................. 75

a) The Overall Results ............................................ 75

b) The Individual Elements .......................................... 83

i) Carbon ................................................. 83

ii) Sodium …................................................ 87

iii) Silicon and the $\alpha$-Elements .............................. 87 
iv) Vanadium ......................................... 88

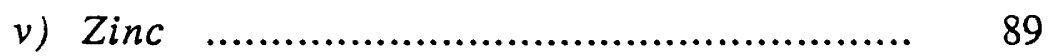

vi) Strontium …........................................... 89

vii) The Heavy Neutron-Capture Elements .............. 89

V. THE SMC STAR AV 369 ....................................... 91

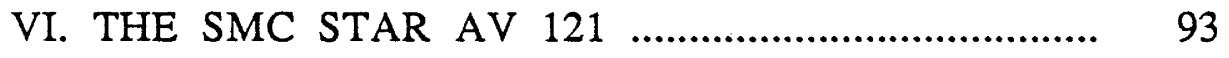

VII. SUMMARY ….................................................. 96

REFERENCES _............................................... 98

Abundances of the Heavy Elements in the Magellanic Clouds.

II. H II Regions and Supernova Remnants ....................... 106

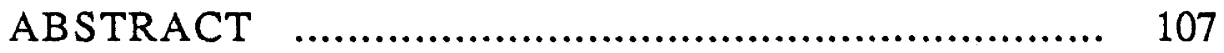

I. INTRODUCTION ............................................... 108

II. OBSERVATIONS AND REDUCTIONS ....................... 109

a) Data Acquisition ................................................. 109

b) Reddening ................................................ 115

c) Line Strengths ................................................ 120

III. THE PHYSICAL PARAMETERS - HII REGIONS ......... 140

IV. THE DETAILED MODELING - H II REGIONS ............ 144

V. RESULTS - H II REGIONS ……............................ 151

a) Helium ....................................................... 151

b) Nitrogen ..................................................... 151

c) Oxygen ..................................................... 152

d) Neon ....................................................... 153

e) Magnesium ..................................................... 154

f) Sulphur .................................................... 155

g) Chlorine ..................................................... 155

h) Argon ................................................. 155

VI. THE DETAILED MODELING OF THE SUPERNOVA

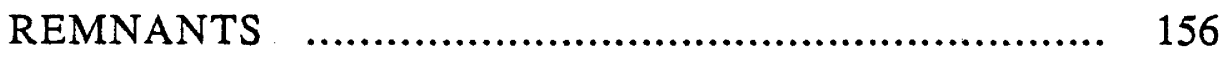


a) The optical spectra ............................................ 156

b) The UV spectra ............................................ 165

VII. THE HEAVY METALS ...................................... 170

VIII. OVERALL RESULTS …................................... 180

REFERENCES _................................................ 190

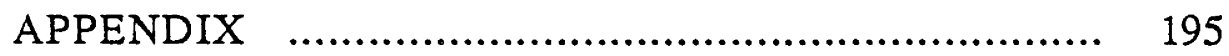

Abundances of the Heavy Elements in the Magellanic Clouds.

III. Interpretation of Results ..................................... 200

ABSTRACT ….............................................. 201

I. INTRODUCTION …......................................... 203

II. STAR FORMATION MODELS ……......................... 204

a) The Structural Evolution ......................................... 204

b) The Nucleosynthesis Prescriptions .......................... 210

c) The chemical evolution ...................................... 213

III. THE RELATIVE ABUNDANCE DISTRIBUTION OF THE ELEMENTS …............................................ 215

a) Carbon, nitrogen, and oxygen ................................ 215

b) The $\alpha$-elements ................................................ 220

c) Neon and Argon ............................................... 223

d) The odd elements ................................................ 225

e) The even Fe-peak elements ..................................... 226

f) The neutron-capture elements ................................. 229

g) The overall abundance pattern ............................... 237

IV. DISCUSSION ............................................... 243

a) Star formation .................................................. 243

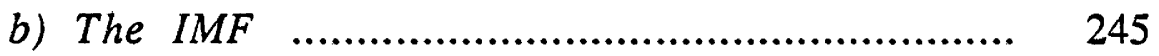

c) Radial Inflow in the Galaxy .................................. 245

V. SUMMARY AND FUTURE WORK ............................ 248

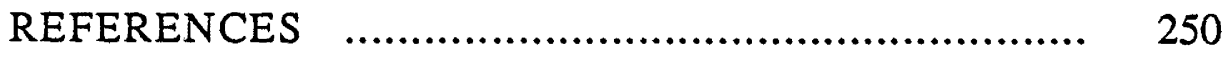




\section{INTRODUCTION}

The Magellanic Clouds are the two closest galaxies to our own, the Small Cloud (SMC) being just $70 \mathrm{kpc}$ distant, and the Large Cloud (LMC) being even closer at a distance of $50 \mathrm{kpc}$. They are more than ten times closer to us than M31, the nearest spiral galaxy, and as such, are the only galaxies available for detailed studies that can provide a link between the Milky Way Galaxy and all the more distant galaxies. By very good luck, these two galaxies are also far out of the plane of the Galaxy and are thus easily observable with little dust obscuration.

Perhaps the most fundamental question one can ask of a galaxy is, how did it evolve to the state in which we now see it? This is the question we ask of the Magellanic Clouds. We attempt to answer the question by determining the presentday detailed elemental abundance patterns in the Clouds, and discussing the star formation that must have occurred in the past in order for these elements to have been synthesized in the ratios we now see. This will vastly improve our understanding of how galaxies differ one to another, as it will extend the present data-base of one galaxy (the Milky Way) for which abundance details are known, by a factor of three, spread over a range of age, metallicity, systemic mass, and galactic type. In short, much more accurate galactic evolutionary models will become possible for all galaxies in general, by a firm knowledge of the detailed abundances in these three systems.

Critical to this investigation is the finding of Pagel et al. (1978) that both of the Magellanic Clouds appear to be well mixed systems (probably due to the rotation of the central bars). So that a measure of the elemental abundances at any one point in the Clouds should reflect the global abundances of the whole Cloud. This enables the total set of abundances to be investigated through measuring only a modest number of objects. 
Even at the distance of the Magellanic Clouds, the types of object potentially capable of providing detailed abundance information are severely limited, but these are reduced still further by the necessity of limiting the study to that of the present day interstellar medium (ISM). This restriction is imposed by the light elements $\mathrm{He}, \mathrm{N}, \mathrm{O}, \mathrm{Ne}$ and $\mathrm{Ar}$, since these are only observable in young objects. Three types of objects were therefore chosen for study, all of which are so young that they effectively provide information on the present day elemental abundances of the ISM in each of the Clouds.

In order to measure the heavier elements (as well as several of the lighter elements, particularly $\mathrm{C}$ and $\mathrm{Mg}$ ), a sample $\mathrm{F}$-supergiants was chosen for study in each of the Clouds. True supergiants are very young objects, since by definition they have zero-age main-sequence (ZAMS) masses greater than $10-12 M_{\odot}$. In the past the detectors used for recording spectra were far less sensitive than they are today, and only the brightest stars, the A supergiants, were available for study. This resulted in a poor determination of their physical parameters and a correspondingly wide divergence in estimates of their abundances by different investigators (see for instance Przybylski 1972, and Wolf 1973). With the larger telescopes and the latest generation of sensitive detectors, cooler and less luminous supergiants could be studied in detail than was the case in the pioneering studies.

In the first paper of this thesis, a sample of $8 \mathrm{~F}$-supergiants were chosen for study in each of the Magellanic Clouds. Although the luminosity class Ib was preferred, several brighter stars were included to extend the sample, since many of the Ib stars were too faint for adequate signal-to-noise data to be obtained in the time available. The spectral class $F$ was chosen by preference because; these stars typically display a large range of weak and strong metal absorption lines in the region of the spectrum over which the detectors are most sensitive, the temperatures are more readily determined, and the continuum could still be placed accurately. 
Observations included broad-band $B V R I$, and medium waveband Strömgren photometry, as well as high dispersion spectroscopy. The photometry was used to determine the physical parameters of the stars, and fine analyses of the spectra were performed to determine the elemental abundances.

In the second paper of this thesis, a sample of small bright circular $\mathrm{H} \mathrm{II}$ regions was chosen for observation in each of the Clouds, in order to obtain the abundances of the lighter elements. Many of these regions have been studied previously in the literature (Pagel et al. 1978 and references therein, Dennefeld and Stasinska 1983), thus valuable comparisons could be made. Few of the studies, however, were extended beyond the optical limit of around $7500 \AA$. Using a CCD detector, observations were made beyond this limit to around $10,000 \AA$, thereby including several potentially valuable lines, otherwise unattainable.

Peimbert and Costero (1969) first developed a method for deriving abundances in $\mathrm{H} \mathrm{II}$ regions through the calculation of semiempirical ionization correction factors in order to predict the contributions to the abundances from unseen ionization species. This method has been used with some success to derive abundances in a number of galaxies (Peimbert and Torres-Peimbert 1974, 1976; Dufour 1975, 1977; Smith 1975; Pagel et al. 1978; Lequeux et al. 1979). More recently, theoretical ionization models have improved sufficiently to have been employed successfully in the direct determination of abundances in individual $\mathrm{H}$ II regions (Shields and Searle 1978; Dufour et al. 1980; Dufour, Shields, and Talbot 1982).

In this study, the general purpose modelling code MAPPINGS (Binette, Dopita, and Tuohy 1985, Evans and Dopita 1985), expressly written to model the global properties of photoionized and shocked regions of gas, was used to analyze the data. The global properties are modelled in this code since for most galaxies the objects available for study are unresolved. In order to integrate over resolvable objects, the telescope was continuously scanned across the object during an 
exposure. Photoionized regions are assumed to be spherically symmetric in the modelling code, hence a selection criterion for the $\mathrm{H} I I$ regions was that they be circular in shape. Bright H II regions were chosen for study to obtain the highest signal-to-noise data possible in the limited amount of telescope time available.

Until now there has been no way of determining the zero-point difference between the absolute abundance scales provided by the supergiants and the $\mathrm{H} \mathrm{II}$ regions, since the two types of object have no observable elements in common. This difficulty was overcome by chosing for study a sample of mature supernova remnants in each of the Clouds. Once a supernova blast wave has swept up a greater mass of gas than that ejected from the star itself, the spectra from the recombining gas should reflect only the abundances of the ISM with little contamination from the material ejected. The collisional excitation mechanism of the gas results in a somewhat different spectrum to that derived from photoionized nebulae, and significantly, includes many forbidden $\mathrm{Fe}$ II lines, as well as several forbidden $\mathrm{Fe}$ III, Ca II, $\mathrm{Cr}$ II and Ni II lines. The potential for connecting the disparate abundance sets derived from H II regions and supergiants is therefore available from sufficiently mature supernova remnants (SNRs).

The main problem with determining abundances from shock excited nebulae is the complexity of the physics in the post shock region. The effects of metallicity, shock conditions and non-equilibrium conditions on the optical spectra must be derived through detailed modelling. The first major step taken in this direction was the classic work of Cox (1972), and since then great advances have been made in the sophistication of the models (see for example Dopita 1977, Raymond 1979, Shull and McKee 1979, Butler and Raymond 1980, and Dopita et al. 1984)

In order to model the SNRs in this study, use was again made of the MAPPINGS code. This ensured consistency between the modelling methods employed in analysis of the $\mathrm{H} I I$ regions and the SNRs. The heavy metals, 
however, were not included specifically in the modelling code, and for these it was necessary to perform a differential abundance analysis with a sample of Galactic Herbig-Haro $(\mathrm{H}-\mathrm{H})$ objects. These objects are also thought to be shocked gaseous objects (Schwartz 1975), and since they are relatively close to the Sun, they have the added advantage that they should have abundances not far removed from solar abundance (see Brugel, Böhm and Mannery 1981 for confirmation of this). In this way the full range of abundances were analyzed in a consistent manner.

The third and final paper describes chemical evolutionary models for the Magellanic Clouds and compares them with a similar type of model for the Galaxy. The abundances derived from the previous two papers are examined for any sign of correlations existing between different abundance ratios, and are discussed in relation to the present day understanding of chemical evolution and the models developed to describe the Clouds. This paper is basically a conclusion to the previous two papers, and as such acts on its own as a conclusion to this thesis. 


\section{REFERENCES}

Binette, L., Dopita, M. A., and Tuohy, I. R. 1985, Ap. J., 297, 476.

Brugel, E. W., Böhm, K. H., and Mannery, E. 1981, Ap. J. Suppl., 47, 117.

Butler, S. E., and Raymond, J. C. 1980, Ap. J., 240, 680.

Cox, D. P., 1972, Ap. J., 178, 143.

Dennefeld, M., and Stasinska, G. 1983, Astr. Ap., 118, 234.

Dopita, M. A. 1977, Ap. J. Suppl., 33, 437.

Dopita, M. A., Binette, L., D'Odorico, S, and Benvenuti, P. 1984, Ap. J., 276, 653.

Dufour, R. J. 1975, Ap. J., 195, 315. . 1977, Ap. J., 216, 706.

Dufour, R. J., Shields, G. A., and Talbot, R. J. 1982, Ap. J., 252, 461.

Dufour, R. J., Talbot, R. J., Jensen, E. B., and Shields, G. A. 1980, Ap. J., 236, 119.

Evans, I. N., and Dopita, M. A. 1985, Ap. J. Suppl., 58, 125.

Lequeux, J., Peimbert, M., Rayo, J. F., Serrano, A., and Torres Peimbert, S. 1979, Astr. Ap., 80, 155.

Pagel, B. E. J., Edmunds, M. G., Fosbury, R. A. E., and Webster, B. L. 1978, M.N.R.A.S., 184, 569.

Peimbert, M., and Costero, R. 1969, Bol. Obs. Tonantzintla y Tacubaya, 5, 3.

Peimbert, M., and Torres-Peimbert, S. 1974, Ap. J., 193, 327. 1976, Ap. J., 203, 581.

Przybylski, A 1972, M.N.R.A.S., 159, 155.

Raymond, J. C. 1979, Ap. J. Suppl., 39, 1.

Schwartz, R. D. 1975, Ap. J., 195, 631.

Shields, G. A., and Searle, L. 1978, Ap. J., 222, 821.

Shull, J. M., and McKee, C. F. 1979, Ap. J., 227, 131. 
Smith, H. E. 1975, Ap. J., 199, 591.

Wolf, B. 1973, Astr. Ap., 28, 335. 
Abundances of the Heavy Elements in the Magellanic Clouds . I. Metal Abundances of F-type Supergiants.

\author{
Stephen C. Russell \\ and
}

Michael S. Bessell

Mount Stromlo and Siding Spring Observatories, Institute of Advanced Studies, The Australian National University.

Received: 1988 September 16

Accepted: 1988 December 1

To appear in Astrophysical Journal, 1989, August. 


\begin{abstract}
We present an analysis of high-resolution spectroscopy for eight F-type supergiants in each of the Magellanic Clouds together with new Strömgren $u v b y$ and Cousins BVRI photometry for these stars. Most of the stars are less luminous than any studied previously in the literature, thus ensuring more reliable measures of their physical parameters. The following conclusions were reached:

1. The mean iron abundance for the $\mathrm{SMC}$ is $[\mathrm{Fe} / \mathrm{H}]=-0.65 \pm 0.2 \mathrm{dex}$.

2. The mean iron abundance for the $\mathrm{LMC}$ is $[\mathrm{Fe} / \mathrm{H}]=-0.30 \pm 0.2 \mathrm{dex}$.

3. The abundances of the stars in both Clouds appear relatively uniform, although there is a suggestion of a scatter in abundances of perhaps $\sim 0.2$ dex in addition to the observational uncertainties.

4. Overall, the abundances of the elements studied in this paper, relative to iron, are very similar in the Magellanic Clouds and in Canopus.

5. The carbon-to-iron abundance, in particular, is the same in both Clouds as in Canopus. This is highly significant since previous results, from UV spectra of $\mathrm{H}$ II regions, have indicated that Carbon is highly underabundant in both Clouds.

6. Vanadium is somewhat overabundant in the LMC.

7. Heavy neutron-capture elements, $\mathrm{Nd}$ and $\mathrm{Sm}$, are overabundant in both Clouds, thus supporting the trends found by Spite, Spite and François (1988) for three stars in the SMC.
\end{abstract}




\section{INTRODUCTION}

The detailed study of the abundances of elements in neighboring galaxies is essential for our understanding of star formation in galaxies. It is the accumulation of all previous stellar debris that determines the exact abundance pattern of heavy elements in the inter-stellar medium (ISM) of a particular galaxy. Some stars are capable of producing more of a certain element than are other stars. Therefore working backwards from a measured abundance pattern, one is able to put constraints on the numbers of each type of star.

Most galaxies are too far away for us to make any attempt at measuring the detailed abundances of their constituent stars. For these galaxies then, we must depend upon measurements of the integrated light of many stars in order to make any comment on their elemental abundances. This is far from ideal since such a random selection of stars is likely to have a large range in age as well as in physical type. Clearly, in order to understand what we are in fact observing in these more distant galaxies, we first need to analyze individual stars in as many neighboring galaxies as we can.

The Magellanic Clouds are ideal in this respect. They are the closest galaxies to our own; close enough for high-dispersion spectra to be obtained of the brighter stars, and for photometry to be obtained well down the main sequence. In addition, they are far out of the plane of the Galaxy and thus little affected by reddening, and their distances are by now well determined.

This is the first of three papers that present the results of a consistent survey of heavy element abundances in the Magellanic Clouds. The survey is consistent in the sense that the whole range of elements, from carbon, nitrogen and oxygen to some of the heaviest elements observed in stellar spectra, is analyzed in a consistent manner. The broad aim of the project is to address the two problems mentioned above: 
1. To make an attempt at unraveling the star formation history of the Magellanic Clouds.

2. To provide a basis for comparative abundance analyses of more distant galaxies.

In this paper we shall concentrate on the abundance analysis of F-type supergiants. Stellar spectra of F- and G-type supergiants have in the past provided information only on the higher mass elements and have had no overlap with those elements derived from $\mathrm{H}$ II regions, the other well-known abundance indicators. The latter provide information only on the lightest elements. In order to bridge the gap between the two methods and thus provide a consistent abundance scale, we shall, in Paper II, present results on the abundance analyses of supernova remnants in the Magellanic Clouds (as pioneered by Dopita 1977 and Dopita et al. 1984), in addition to more precise studies of $\mathrm{H}$ II regions.

Supergiant stars are, by definition, massive objects. We take them to be stars with a zero-age main-sequence (ZAMS) mass greater than about $10 M_{\odot}$. They are therefore very young objects, and, as such, they must reflect the current abundance distribution of the local ISM. Fortunately, both Clouds are stirred by the rotation of their central bars, so that the abundances of the local ISM in any particular place in either Cloud should reflect the abundances in the ISM of the Cloud as a whole (see Pagel et al. 1978). This concept has been challenged in the past by the discovery of a star in the field of the SMC by Russell, Bessell, and Dopita (1988a, b), that appeared to have an abundance far below the norm. We show here, in a more complete study of that star, that although the abundances of this star are indeed low, they are not not significantly different from those of other stars studied herein or in the literature. Another piece of evidence against the concept of complete mixing of the global ISM in the Clouds comes from a study by Spite et al. (1986) of a red supergiant in the very young SMC cluster, NGC 330 (see Carney, Janes, and Flower, 1985). If Spite et al. (1986) are correct, then their star (A7) is nearly four 
times less metal-rich than the average for the field of the SMC. Recently, however, Reitermann et al. (1988) have studied a near-main-sequence B star in NGC 330, and have found it to have normal or enhanced abundances for the SMC. It seems hardly credible that two stars in the same cluster could have such wildly different abundances. We shall leave further discussion of this to Paper III, where we shall combine the results of Papers I and II into a holistic view of the two Clouds and draw our conclusions.

Previous analyses of supergiants in the Magellanic Clouds (see Tables 1 and 2) have, until this decade, been highly unreliable. Iron abundances, even for the same star measured by independent investigators, have differed by up to 0.7 dex. This was largely due to the stars chosen for measurement being the brightest, most extreme stars in each galaxy. For such stars, many of the classical assumptions (for example: local thermal equilibrium [LTE], hydrostatic equilibrium, plane-parallel atmosphere) made in modeling their atmospheres break down. In addition, there are no Galactic counterparts to these stars with which comparisons could be made. Recent advances in instrumentation now allow the fine analysis of less extreme stars. 
TABLE 1

SMC Abundances from the Literature

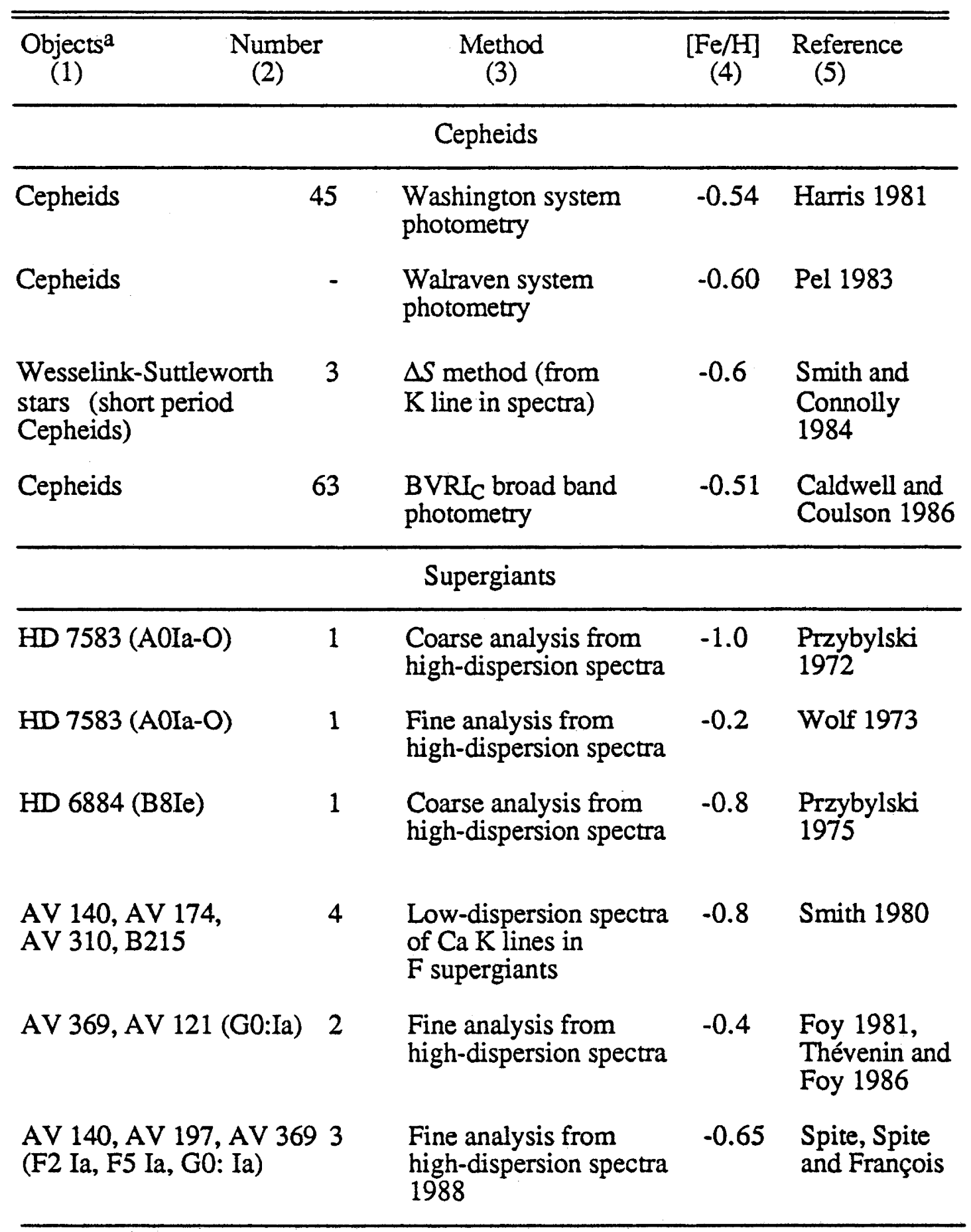

a AV = Azzopardi and Vigneau 1975; B = Basinski, Bok and Bok 1967 
TABLE 2

LMC Abundances from the Literature

\begin{tabular}{|c|c|c|c|c|}
\hline $\begin{array}{l}\text { Objectsa }^{a} \\
\text { (1) }\end{array}$ & $\begin{array}{l}\text { Number } \\
\text { (2) }\end{array}$ & $\begin{array}{l}\text { Method } \\
\text { (3) }\end{array}$ & $\begin{array}{c}{[\mathrm{Fe} / \mathrm{H}]} \\
(4)\end{array}$ & $\begin{array}{l}\text { Reference } \\
\text { (5) }\end{array}$ \\
\hline \multicolumn{5}{|c|}{ Cepheids } \\
\hline Cepheids & - & $\begin{array}{l}\text { Walraven system } \\
\text { photometry }\end{array}$ & -0.20 & Pel 1983 \\
\hline Cepheids & 73 & $\begin{array}{l}B V R I_{C} \text { broad-band } \\
\text { photometry }\end{array}$ & -0.18 & $\begin{array}{l}\text { Caldwell and } \\
\text { Coulson } 1986\end{array}$ \\
\hline \multicolumn{5}{|c|}{ Supergiants } \\
\hline $\begin{array}{l}\text { HD } 33579 \\
\text { (A3 Ia-O) }\end{array}$ & 1 & $\begin{array}{l}\text { Coarse analysis from } \\
\text { high-dispersion spectra }\end{array}$ & -0.2 & Przybylski 1968 \\
\hline $\begin{array}{l}\mathrm{HD} 33579 \\
(\mathrm{~A} 3 \mathrm{Ia}-\mathrm{O})\end{array}$ & 1 & $\begin{array}{l}\text { Coarse analysis from } \\
\text { medium-dispersion } \\
\text { spectra }\end{array}$ & 0.0 & $\begin{array}{l}\text { Wares, Ross and } \\
\text { Aller } 1968\end{array}$ \\
\hline $\begin{array}{l}\text { HD } 33579 \\
(\text { A3 Ia-O) }\end{array}$ & 1 & $\begin{array}{l}\text { Fine analysis from } \\
\text { high-dispersion spectra }\end{array}$ & -0.2 & Wolf 1972 \\
\hline $\mathrm{HD} 32034$ (B9 Ia) & 1 & $\begin{array}{l}\text { Coarse analysis from } \\
\text { high-dispersion spectra }\end{array}$ & -0.8 & Przybylski 1971 \\
\hline $\begin{array}{l}\mathrm{HD} 269781 \\
\text { (A0 Iae) }\end{array}$ & 1 & $\begin{array}{l}\text { Coarse analysis from } \\
\text { high-dispersion spectra }\end{array}$ & -0.7 & Przybylski 1975 \\
\hline HD 271182 (F8 Ia) & ) 1 & $\begin{array}{l}\text { Coarse analysis from } \\
\text { high-dispersion spectra }\end{array}$ & +0.2 & Przybylski 1979 \\
\hline HD 268759 (G4 Ia) & ) 1 & $\begin{array}{l}\text { Spectrum synthesis from } \\
\text { high-dispersion spectra }\end{array}$ & -0.2 & $\begin{array}{l}\text { Fry and Aller } \\
1975\end{array}$ \\
\hline $\begin{array}{l}\text { G47, G221, G247, } \\
\text { G317, G396, G403 }\end{array}$ & 6 & $\begin{array}{l}\text { Low-dispersion spectra } \\
\text { of } \mathrm{Ca} \mathrm{K} \text { lines in }\end{array}$ & -0.3 & $\begin{array}{l}\text { Smith } 1980 \\
\text { F supergiants }\end{array}$ \\
\hline $\begin{array}{l}\text { F- and G-type } \\
\text { supergiants }\end{array}$ & 47 & $\begin{array}{l}\text { Walraven system } \\
\text { photometry }\end{array}$ & -0.18 & $\begin{array}{l}\text { van Genderen, } \\
\text { Driel, Greidanus } \\
1986\end{array}$ \\
\hline
\end{tabular}

a $G=$ Fehrenbach and Duflot 1970, "Liste G." 
Foy (1981) and Thévenin and Foy (1986) each studied a different G0: Ia star in the SMC, and although these two quite similar stars are about a magnitude fainter than any previous star analyzed, their physical parameters, and therefore their abundances, are still only poorly determined (we shall discuss these stars in more detail later on). Recently, however, Spite, Spite, and François (1988) have reappraised the star studied by Foy (1981) (see Fig. 1 where the two estimates of luminosity and temperature for the same star are shown connected by a dotted line) plus two F Ia supergiants nearly a full magnitude less luminous still. The latter two stars are moderate enough for their physical parameters to be reasonably well determined and they indicate an iron deficiency of $[\mathrm{Fe} / \mathrm{H}]=-0.64$ for the SMC (where the generally accepted convention $[\mathrm{Fe} / \mathrm{H}]=\log (\mathrm{Fe} / \mathrm{H})_{s t a r}-\log (\mathrm{Fe} / \mathrm{H})_{\odot}$ is used here). This value is in good accord with that derived from studies of Cepheids (see Table 1) and a preliminary analysis of our own results (Russell, Bessell, and Dopita 1988a, b).

Unfortunately, no similar work on more moderate stars in the LMC, besides our own preliminary analysis (Russell, Bessell, and Dopita 1988a, b), has been carried out to our knowledge. The evidence from the literature, however (see Table 2), points toward an iron deficiency of $[\mathrm{Fe} / \mathrm{H}]$ between -0.1 and -0.3 .

For this survey we chose for analysis the faintest $F$ supergiants possible, while still achieving spectra with reasonable signal-to-noise ratios (see Fig. 2). Figure 1 shows our initial selection of stars on a graph of bolometric magnitude versus effective temperature, with our Galactic standards and several stars studied in the literature for comparison. The magnitudes of our stars range down from the faintest stars studied by Spite, Spite, and François (1988) to stars 1.8 mag fainter. This selection ensures that the physical parameters of these stars can be derived with some accuracy. The F spectral types were chosen to ensure that there was a plentiful 
Figure 1 Bolometric magnitude against effective temperature for stars from this work, plus several from the literature (Spite et al. refers to Spite, Spite, and François 1988, and F and TF refer to Foy [1981] and Thévenin and Foy [1986] respectively). Included on this diagram are evolutionary tracks for 9,15 , and $30 M_{\odot}$ taken from case B of Maeder 1981. Also included are the approximate lines (dashed) of constant gravity: $\log g=0,1$, and 2 . The dotted line joins the lower temperature estimate of AV 369 by Foy (1981) to the higher temperature estimate by Spite, Spite, and François (1988). 
鄯号

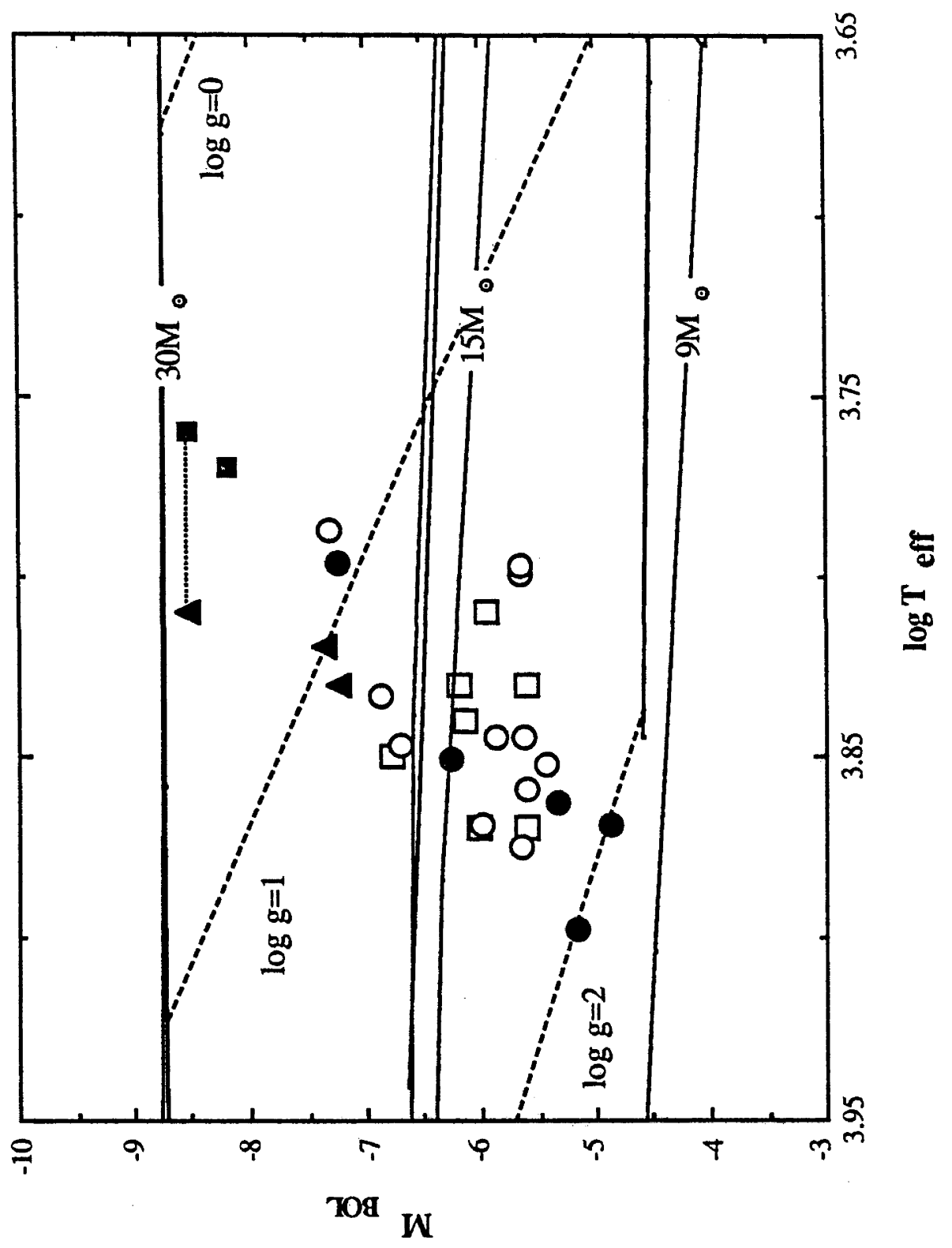


Figure 2 (a) A part of a typical spectrum (in this case, AV 401) in the region of Ba II line at $4554.0 \AA$. (b) The same part of the spectrum as in (a), but for the low abundance star AV 79. 

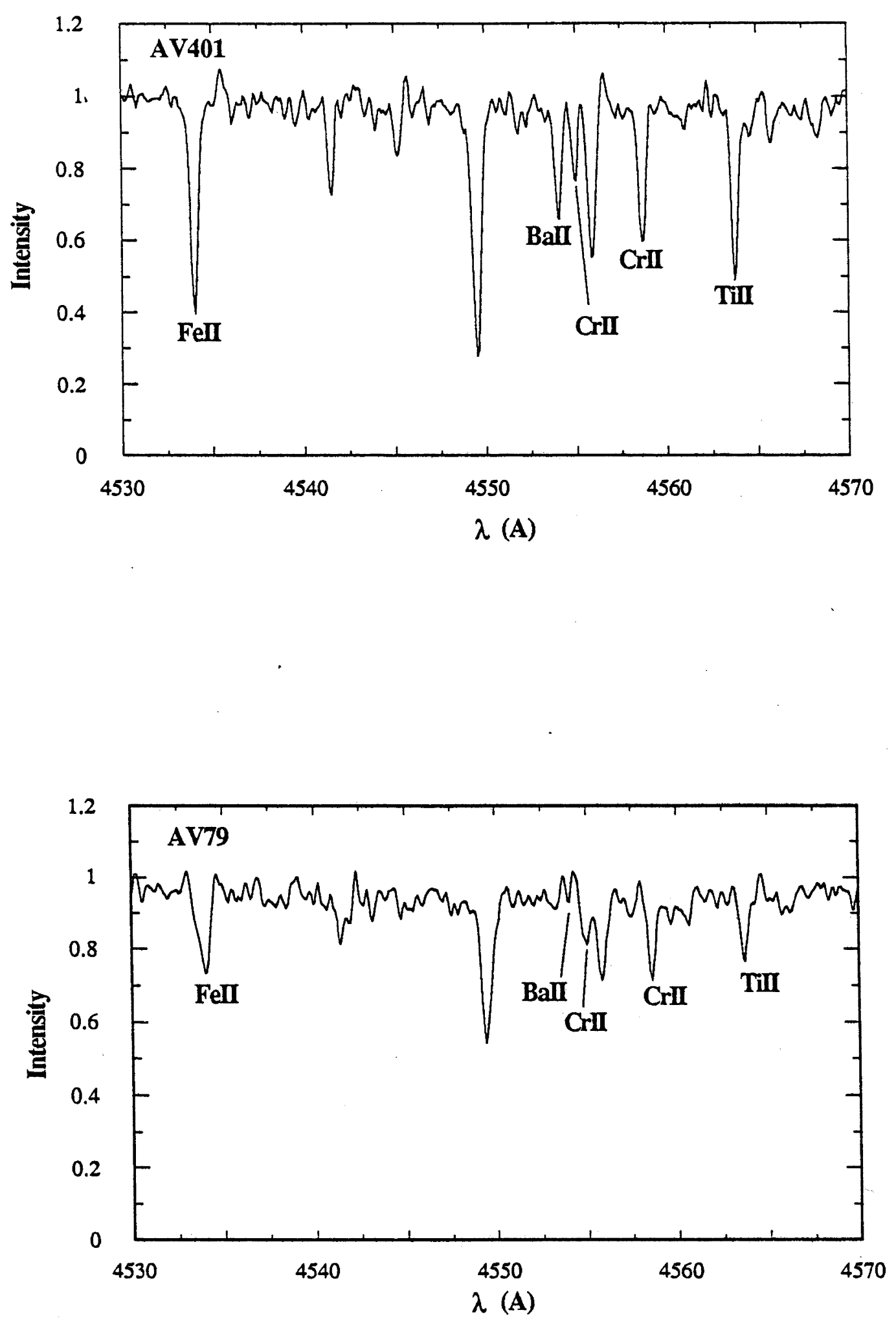
supply of metal lines in the blue part of the spectrum where we wished to work (due to the response of the detectors), while still allowing adequate placement of the continuum. Cooler stars than these would have suffered from line crowding but, more importantly, from the effects of molecular opacity which were not allowed for in the Kurucz (1979) atmospheric models we were using.

In the next section we shall present the results of our photometry and use these to make a coarse estimate of the physical parameters of our program stars. Section III will present our spectrographic observations, and § IV, the overall results of our abundance analysis. We shall follow this in $\S \S \mathrm{V}$ and VI with a discussion of the rather luminous SMC stars AV 369 and AV 121 respectively. Finally we shall summarize our conclusions in § VII.

\section{PHOTOMETRY}

\section{a) The Observations}

The stars we chose for our observing program (see Table 3) were taken from the list of Azzopardi and Vigneau (1975) for the SMC and mostly from the list of Ardeberg et al. (1972) for the LMC. In the latter case, all of the stars chosen for observation in the LMC had been given a number in "Liste G" of Fehrenbach and Duflot (1970), and for consistency, it is these numbers we shall use throughout this series of papers. The detection of variability (see col. [7] of Table 3) for these stars depended upon the survey of Grieve and Madore $(1986 a, b)$. This only includes a small number of stars from our own survey, and therefore our program should include several more variable stars that have not yet been identified as such. This 
TABLE 3

The Program Starsa

\begin{tabular}{|c|c|c|c|c|c|c|}
\hline $\begin{array}{l}S \\
\text { Star } \\
(1)\end{array}$ & $\begin{array}{l}\text { Tpectral } \\
\text { Typeb } \\
(2)\end{array}$ & $\begin{array}{l}V \\
(3)\end{array}$ & $\begin{array}{l}\text { R.A. (1950) } \\
(4)\end{array}$ & $\begin{array}{l}\text { 0) Decl. D } \\
(5)\end{array}$ & $\begin{array}{l}\text { Other } \\
\text { Designations } \\
\text { (6) }\end{array}$ & $\begin{array}{c}\text { Notes }^{\mathrm{C}} \\
\text { (7) }\end{array}$ \\
\hline \multicolumn{7}{|c|}{ SMC } \\
\hline AV 79 & F2 & 13.71 & $00^{\mathrm{h}} 48^{\mathrm{m}} 54^{\mathrm{s}}$ & $-73006 ' 22^{\prime \prime}$ & & Faint companion \\
\hline AV 107 & F5 & 13.63 & 005000 & -725213 & & Faint companion \\
\hline AV 121 & G0 & 11.31 & 005038 & -725557 & Dac 1-11 & Faint companion \\
\hline AV 127 & F2 & 12.52 & 005048 & -732953 & Flo 256 & Variable \\
\hline AV 198 & F5 & 13.41 & 005620 & -723542 & & Variable \\
\hline AV 305 & F5 & 12.86 & 010056 & -723126 & Flo 439 & Variable \\
\hline AV 323 & F2 & 13.24 & 010124 & -724620 & & \\
\hline AV 401 & F2 & 13.20 & 010442 & -725133 & & \\
\hline \multicolumn{7}{|c|}{ LMC } \\
\hline G39 & F8 I & 13.22 & 045158 & -685806 & Ard 25 & \\
\hline G95 & F0 I & 13.08 & 045615 & -694809 & & \\
\hline G104 & F2 I & 13.13 & 045632 & -665706 & Ard 68 & \\
\hline G131 & F0 I & 13.30 & 045823 & -670400 & Ard 86 & \\
\hline G144 & F0 I & 12.77 & 045910 & -693000 & Ard 91 & \\
\hline G231 & F0 I & 12.69 & 051128 & -682706 & Ard 164 & \\
\hline G274 & F6: Ia & 12.26 & 052220 & -665746 & Ard 207 & \\
\hline G317 & F5 Ia & 11.45 & 052841 & -690205 & Ard 246 & \\
\hline G439 & F6 Ia & 11.86 & 054224 & -682848 & Ard 361 & \\
\hline G440 & F0:I & 13.17 & 054225 & -702648 & Ard 362 & \\
\hline G470 & FOI & 13.00 & 054703 & -684726 & & \\
\hline
\end{tabular}

a Ard = Ardeberg et al. 1972; AV = Azzopardi and Vigneau 1975; Dac = Dachs 1970; Flo = Florsch 1972; G = Fehrenbach and Duflot 1970, "Liste G";

b Spectral types and magnitudes for the SMC are from Azzopardi and Vigneau 1975; Spectral types and magnitudes for the LMC are from Rousseau et al. 1977. c Variability, if indicated, was detected by Grieve and Madore (1986b). 
variability is, however, only small ( $0.05-0.1$ magnitudes) and should not affect the physical parameters of the stars (see Osmer 1972).

The primary photometry we used for our program stars was in the mediumbandwidth uvby Strömgren system (see Russell, Bessell, and Dopita 1987 for a fuller discussion of this and for a presentation of preliminary results). This was chosen because it supplies more information than the broad-band $U B V$ system and the results are easier to compare with theoretical calculations. Unfortunately, there is as yet no calibration to determine the reddening for yellow supergiants with this system. Thus additional broad-band Cousins BVRI photometry was undertaken to establish a reliable measure of the reddening.

All photometric observations were carried out on the $2.3 \mathrm{~m}$ telescope at Siding Spring (in Australia) using the TCC (two channel chopper; see Bessell, in preparation) and two gallium arsenide detectors (or at times an extended S20, when one of the other detectors was not available). The observations were made during the 1985,1986 and 1987 observing seasons and were reduced to the standard system using stars drawn from the catalogs of Graham and Slettebak (1973), Philip and Philip (1973), Kilkenny and Hill (1975), Kilkenny, Hill, and Brown (1977), and Olsen (1983). Reduction was carried out using the photometric reduction package SPIDER developed at Mount Stromlo Observatory by A. Collier and B. Green (adapted to Strömgren photometry by S. Russell and B. Green).

The results of the photometry are given in Table 4, where the Strömgren indices are given in columns (2)-(4), and the broad-band indices in columns (7)(10). Columns (5) and (11) give the standard deviations from the mean of the Strömgren and broad-band indices, respectively, in units of $0.001 \mathrm{mag}$, for each of the columns to their left. Column (6) gives the number of times the star was observed with the Strömgren filter set, and, as can be seen, at least four measures 
TABLE 4

Photometery

\begin{tabular}{|c|c|c|c|c|c|c|c|c|c|}
\hline $\begin{array}{c}\text { Star } \\
\text { (1) }\end{array}$ & $\begin{array}{l}b-y \\
(2)\end{array}$ & $\begin{array}{l}m_{1} \\
(3)\end{array}$ & $\begin{array}{l}c_{1} \\
(4)\end{array}$ & $\begin{array}{c}\Delta \\
(5)\end{array}$ & $\begin{array}{l}n B-V \\
(6) \quad(7)\end{array}$ & $\begin{array}{r}V-R \\
(8)\end{array}$ & $\begin{array}{l}V-I \\
(9)\end{array}$ & $\begin{array}{l}R-I \\
(10)\end{array}$ & $\begin{array}{c}\Delta \\
(11)\end{array}$ \\
\hline \multicolumn{10}{|c|}{ Galactic Standards } \\
\hline v Car & 0.197 & 0.074 & 1.403 & & 20.28 & 0.192 & 0.415 & 0.223 & \\
\hline$\alpha \operatorname{Car}$ & 0.110 & 0.128 & 1.512 & & $\begin{array}{ll}4 & 0.16\end{array}$ & 0.125 & 0.230 & 0.105 & \\
\hline$\alpha$ Lep & 0.139 & 0.148 & 1.504 & & $14 \quad 0.21$ & 0.154 & 0.321 & 0.167 & \\
\hline HR3496 & 0.394 & 0.054 & 1.534 & & 20.56 & 0.366 & 0.757 & 0.391 & \\
\hline$\delta \mathrm{CMa}$ & 0.375 & 0.322 & 0.929 & & $\begin{array}{ll}6 & 0.67\end{array}$ & 0.362 & 0.671 & 0.309 & \\
\hline \multicolumn{10}{|c|}{ SMC } \\
\hline AV 79 & 0.199 & 0.115 & 1.365 & $17,30,30$ & 60.301 & 0.226 & 0.530 & 0.295 & $38,09,16,11$ \\
\hline AV 107 & 0.229 & 0.140 & 1.435 & $09,13,19$ & 60.361 & 0.235 & 0.567 & 0.324 & $75,18,41,23$ \\
\hline AV 121 & 0.444 & 0.337 & 0.952 & $06,28,35$ & 40.746 & 0.398 & 0.786 & 0.382 & $40,05,13,07$ \\
\hline AV 127 & 0.204 & 0.114 & 1.579 & $23,19,45$ & 110.257 & 0.168 & 0.372 & 0.197 & $31,26,30,06$ \\
\hline AV 198 & 0.292 & 0.139 & 1.362 & $18,25,38$ & 120.426 & 0.284 & 0.603 & 0.313 & $42,23,78,54$ \\
\hline AV 305 & 0.178 & 0.138 & 1.495 & $15,06,27$ & 60.265 & 0.189 & 0.314 & 0.197 & $36,14,20,10$ \\
\hline AV 323 & 0.217 & 0.179 & 1.435 & $13,13,25$ & 60.285 & 0.198 & 0.457 & 0.245 & $15,18,24,05$ \\
\hline AV 401 & 0.152 & 0.119 & 1.638 & $17,31,28$ & 120.218 & 0.139 & 0.328 & 0.181 & $07,11,25,14$ \\
\hline \multicolumn{10}{|c|}{ LMC } \\
\hline G39 & 0.354 & 0.227 & 1.087 & $07,30,27$ & 230.535 & 0.331 & 0.642 & 0.308 & $12,11,60,50$ \\
\hline G95 & 0.184 & 0.149 & 1.439 & $08,12,20$ & 50.256 & 0.176 & 0.378 & 0.197 & $02,08,57,49$ \\
\hline G104 & 0.210 & 0.177 & 1.347 & $18,28,43$ & 120.298 & 0.210 & 0.449 & 0.236 & $35,08,15,10$ \\
\hline G131 & 0.169 & 0.165 & 1.468 & $22,21,28$ & 100.236 & 0.151 & 0.325 & 0.171 & $11,15,53,38$ \\
\hline G144 & 0.207 & 0.129 & 1.560 & $09,21,32$ & 140.268 & 0.189 & 0.395 & 0.199 & $16,11,25,14$ \\
\hline G231 & 0.157 & 0.162 & 1.501 & $08,11,36$ & 140.357 & 0.229 & 0.457 & 0.222 & $06,04,13,17$ \\
\hline G274 & 0.316 & 0.229 & 0.914 & $14,24,13$ & 150.475 & 0.304 & 0.596 & 0.286 & $05,09,21,29$ \\
\hline G317 & 0.234 & 0.179 & 1.429 & $07,03,27$ & 60.357 & 0.216 & 0.450 & 0.227 & $11,02,42,40$ \\
\hline G439 & 0.323 & 0.275 & 1.090 & $09,20,24$ & 120.537 & 0.288 & 0.577 & 0.283 & $23,08,28,21$ \\
\hline G440 & 0.218 & 0.127 & 1.578 & $18,23,40$ & 160.280 & 0.175 & 0.374 & 0.193 & $15,11,42,54$ \\
\hline G470 & 0.131 & 0.125 & 1.527 & $15,28,31$ & 50.171 & 0.114 & 0.278 & 0.160 & $16,06,25,22$ \\
\hline
\end{tabular}

Note.- Standards are as follows: Strömgren ( $\mathrm{C}$ Car, HR3496), Grønbech and Olsen 1976; Strömgren (others), Crawford,Barnes, and Golson 1970; B-V, Blanco et al. 1970; V-R and V-I (HR3496), Fernie 1983 (corrected to Cousins system); V-R and V-I (others), Cousins 1980. 
were made for each star. Any measures with poor internal repeatability have been discarded. Comparing our average errors with those of Osmer (1973), the only other survey of the SMC in the Strömgren system (there are no other surveys in this system for the LMC), we find ours to be at least the same accuracy, and for $b-y$ (the most critical index), of slightly better accuracy. The only star that we have in common with Osmer, is AV 369. Although both $b-y$ and $m_{1}$ agree satisfactorily (to better than $0.02 \mathrm{mag}$ ), our measure of $c_{1}$ is far too low. The definition of $c_{1}$ is

$$
c_{1}=(u-v)-(v-b)
$$

while $m_{1}$ is

$$
m_{1}=(v-b)-(b-y)
$$

Clearly then, the error is due to contamination in the $u$ band ( $3500 \AA$ ) by the close blue companion to AV 369 (as noted in Table 3). In fact, no adequate broad-band photometry was obtained for this star due to this problem, and these colors quoted in Table 4 have been taken directly from Grieve and Madore (1986a). As it turns out, we have reason to believe that the photometry of Osmer is also somewhat affected by the presence of the companion, but this shall be discussed further on.

The errors in the broad-band photometry are comparable to those in the Strömgren photometry due to the far fewer observations made with this filter set (always two or more, however, except in the cases of AV 79 and G104 for which only one self-consistent measurement was made in each case, and the quoted errors are then just a measure of the internal consistency). In comparing the broad-band colors we obtained with those obtained by Grieve and Madore (1986a) for the six stars we shared in common, we obtained the following results:

$$
\Delta(B-V)=0.008 \pm 0.013
$$




$$
\begin{aligned}
& \Delta(V-R)=0.001 \pm 0.025, \\
& \Delta(V-I)=0.003 \pm 0.050
\end{aligned}
$$

(where, in the last case, one discrepant value, AV 107, has been discarded)

In addition to the program stars listed in Table 4, we include the Galactic standard stars used in our abundance analysis, with their photometric indices derived solely from the literature. The references for these are listed at the bottom of the table.

\section{b) Reddenings}

Although the reddenings of the Cloud stars have generally been found to be quite small $(E[B-V] \lesssim 0.1 \mathrm{mag}$; see Caldwell and Coulson 1985), adoption of a mean reddening for each Cloud as a whole has been shown, most recently by Grieve and Madore (1986a) and Shobbrook (1986), to lead to substantial error for individual supergiants. Thus it was decided to measure reddening for each star in our program using the methods of Grieve and Madore (1986a), which, for our purposes, seemed far superior to any other discussed in the literature.

The reddenings of colors can be determined from the adoption of an intrinsic line in a color-color or a spectral type-color diagram. Previous authors (for example Brunet 1975, Isserstedt 1975,1977) have relied upon the intrinsic lines determined from supergiants in our own Galaxy. This necessarily depends on the estimate of a "blue-most envelope" to the supergiants; however, since there are only very few such stars with little or no reddening in our galaxy, the line remains very uncertain. Thus Grieve and Madore (1986a) chose to disregard the Galactic sequences and determine the intrinsic colors from the Cloud supergiants directly. This had the added advantage of making no assumptions as to whether the colors of Cloud supergiants are the same as those in the Galaxy. 
The total reddening for a star in the Magellanic Clouds consists of a component from our own galaxy and one intrinsic to the particular Cloud in question. McNamara and Feltz (1980) determined the average Galactic reddening across the face of each Cloud to be

$$
\begin{aligned}
& E(b-y)_{S M C}=0.013 \pm 0.003 \mathrm{mag}, \\
& E(b-y)_{L M C}=0.024 \pm 0.003 \mathrm{mag} .
\end{aligned}
$$

With these values assumed to be constant, then from the distribution of colors on a color-color or spectral type-color diagram, one can define a bluemost envelope corresponding to those stars having no reddening intrinsic to the Cloud itself. This was done, by Grieve and Madore, for their 81 supergiants in the LMC for the colors $B-V, V-R, V-I$, and $R-I$. The eye fits to the envelopes were also used to define the envelopes for the SMC (with the appropriate foreground reddening corrections), since the fewer number of stars measured in that galaxy made it inappropriate to try and fit its own envelopes. The error that this would introduce was thought by Grieve and Madore to be negligible, since they could find no systematic difference between reddenings derived using the $R$ and $I$ bands (for which any metallicity effect would be very small) and those derived from $B-V$. This point shall be considered in relation to our own data a little further on.

The reddenings for each of the program stars were calculated using the two methods described by Grieve and Madore, and the results are given in Table 5. With the $Q$ method one plots the reddening-free parameters defined by Grieve (1983): 


\section{TABLE 5}

Reddening in Terms of $E(b-y)$

\begin{tabular}{|c|c|c|c|c|c|c|c|c|c|c|}
\hline $\begin{array}{l}\text { Star } \\
(1)\end{array}$ & $\begin{array}{l}\text { Q1 } \\
(2)\end{array}$ & $\begin{array}{l}\mathrm{Q} 2 \\
(3)\end{array}$ & $\begin{array}{l}\text { Q3 } \\
(4)\end{array}$ & $\begin{array}{c}\text { Av. } \\
Q \\
(5)\end{array}$ & $\begin{array}{c}\mathrm{Sp} \\
(B-V) \\
(6)\end{array}$ & $\begin{array}{c}S p_{p} \\
(V-R) \\
(7)\end{array}$ & $\begin{array}{c}\text { Sp } \\
(V-I) \\
(8)\end{array}$ & $\begin{array}{r}\mathrm{Sp} \\
(R-I) \\
(9)\end{array}$ & $\begin{array}{l}\text { Av. Ad } \\
\text { Sp } \\
(10)\end{array}$ & $\begin{array}{l}\text { dopted } \\
\text { (11) }\end{array}$ \\
\hline \multicolumn{11}{|c|}{ SMC } \\
\hline $\mathrm{AV} 79$ & $0.250:$ & $0.236:$ & $0.224:$ & 0.237 & 0.045 & 0.089 & 0.128 & 0.141 & 0.087 & $0.09^{a}$ \\
\hline AV 107 & 0.208 & $0.354:$ & $0.330:$ & 0.297 & 0.004 & 0.029 & 0.088 & 0.130 & 0.063 & $0.06^{a}$ \\
\hline AV 121 & 0.103 & 0.117 & 0.106 & 0.109 & 0.289 & 0.220 & 0.210 & 0.190 & 0.227 & $0.11^{b}$ \\
\hline AV 127 & 0.220 & 0.143 & 0.144 & 0.169 & 0.013 & 0.021 & 0.040 & 0.039 & 0.028 & 0.08 \\
\hline AV 198 & 0.298 & 0.287 & 0.332 & 0.306 & 0.052 & 0.087 & 0.108 & 0.118 & 0.091 & $0.09^{a}$ \\
\hline AV 305 & 0.180 & 0.160 & 0.144 & 0.161 & -0.067 & -0.025 & -0.009 & -0.003 & -0.026 & 0.08 \\
\hline AV 323 & 0.186 & 0.233 & 0.188 & 0.202 & 0.033 & 0.056 & 0.088 & 0.089 & 0.067 & $0.08^{c}$ \\
\hline AV 401 & 0.071 & 0.136 & 0.125 & 0.111 & -0.016 & -0.013 & 0.016 & 0.022 & 0.002 & 0.06 \\
\hline \multicolumn{11}{|c|}{ LMC } \\
\hline G39 & 0.153 & 0.105 & 0.079 & 0.112 & -0.004 & 0.060 & 0.057 & 0.061 & 0.059 & 0.09 \\
\hline G95 & 0.139 & 0.168 & 0.148 & 0.152 & 0.064 & 0.066 & 0.071 & 0.060 & 0.065 & $0.07^{a}$ \\
\hline G104 & 0.194 & 0.220 & 0.184 & 0.199 & 0.043 & 0.071 & 0.083 & 0.079 & 0.069 & $0.07^{a}$ \\
\hline G131 & 0.073 & 0.111 & 0.116 & 0.100 & 0.049 & 0.037 & 0.042 & 0.032 & 0.040 & 0.07 \\
\hline G144 & 0.163 & 0.184 & 0.148 & 0.165 & 0.073 & 0.081 & 0.081 & 0.062 & 0.074 & $0.07^{a}$ \\
\hline G231 & 0.137 & 0.114 & 0.036 & 0.096 & 0.138 & 0.128 & 0.115 & 0.086 & 0.117 & 0.11 \\
\hline G274 & 0.188 & 0.125 & 0.073 & 0.129 & 0.048 & 0.087 & 0.081 & 0.079 & 0.074 & $0.03^{d}$ \\
\hline G317 & 0.092 & 0.101 & 0.046 & 0.080 & 0.064 & 0.054 & 0.064 & 0.054 & 0.059 & 0.07 \\
\hline G439 & 0.025 & 0.044 & 0.040 & 0.036 & 0.094 & 0.069 & 0.071 & 0.076 & 0.078 & 0.06 \\
\hline G440 & 0.082 & 0.105 & 0.126 & 0.104 & 0.081 & 0.065 & 0.069 & 0.055 & 0.068 & 0.09 \\
\hline G470 & 0.051 & $0.157:$ & $0.093:$ & 0.100 & 0.001 & -0.007 & 0.016 & 0.021 & 0.008 & 0.05 \\
\hline
\end{tabular}

Note.- Cols. (2)-(5) are derived from the ( $B-V$ ) Q-method of Grieve and Madore 1986a. Cols. (6)-(10) are derived from the spectral type method of Grieve and Madore 1986a (hence the prefix Sp). A colon after a number implies that the blue most envelope derived by Grieve and Madore 1986a has been extapolated in order to accomodate the data.

a Reddening derived from the $\mathrm{Q}$ method results in an unrealistic log $\mathrm{g}$ for the star, and is therefore discarded in favour of the spectral type method.

$\mathrm{b}$ Reddening derived from the spectral type method results in an unrealistic log $\mathrm{g}$ for the star, and is therefore discarded in favour of the $\mathrm{Q}$ method.

c The reddening is derived only from columns (7)-(9), since the other measures are deemed unreliable.

d Reddening derived from both methods appears too high. Therefore the Galactic reddening is adopted. 


$$
\begin{aligned}
& Q_{1}=(V-R)-0.631(B-V)-0.104(B-V)^{2}, \\
& Q_{2}=(B-V)-0.754(V-I)+0.103(V-I)^{2}, \\
& Q_{3}=(R-I)-0.709(B-V)-0.048(B-V)^{2},
\end{aligned}
$$

and the blue-most envelopes, on a $Q$-color diagram (we used only $(B-V)$ for our color in this case). The reddening is then simply the difference between the $B-V$ of the star and that of the blue-most envelope, holding the $Q$ value constant. The derived $E(B-V)$ reddenings were then converted to $E(b-y)$ using the relation from Crawford (1975) :

$$
E(b-y)=0.74 E(B-V) .
$$

These are given in columns (2),(3), and (4) of Table 5, with their averages in column (5).

In the second method one plots the stars and the blue-most envelope, on a color-spectral type diagram, and derives the reddening for each color in the same manner as above, but holding the spectral type constant. These are then converted to an equivalent $E(B-V)$ using reddening ratios derived from the definitions of the $Q$ parameters above, and thence to $E(b-y)$. The results we obtained using this method are given in columns (6)-(9) of Table 5 , with their averages given in column (10).

The finally accepted values are listed in column (11) of Table 5. It may be noted that there does seem to be a systematic difference between reddenings derived from colors containing the $R$ and $I$ filters and those derived from $B-V$ for our sample of SMC supergiants. This may be due to the fact that they are, on average, slightly later than the stars studied by Grieve and Madore. Whatever the reason, this makes the use of the bluemost envelopes derived from the LMC more suspect and probably accounts for the unrealistic reddenings we obtain for several of our SMC stars using the $Q$ method. Some of the reddenings derived from the $Q$ method for stars in the 
LMC have also been discarded, but the errors are much less severe and are probably due simply to poor photometry.

Table 6 gives the dereddened Strömgren indices in columns (5)-(7), where we have used the reddening ratios from Crawford (1975):

$$
\begin{aligned}
& E\left(c_{1}\right)=0.19 E(b-y), \\
& E\left(m_{1}\right)=-0.33 E(b-y) .
\end{aligned}
$$

This table also gives the derived physical parameters of the stars: $T_{\text {eff }}$ (effective Temperature) in column (8), and $\log g$ (where $g$ corresponds to surface gravity) in column (9). The calculation of these values is discussed in the next section.

\section{c) Photometric Derivation of the Stellar Physical Parameters}

The initial estimates for the physical parameters of our program stars, derived wholly from photometry, are, as mentioned above, given in Table 5. These are based on the theoretical photometric indices for the Strömgren system calculated by Lester, Gray, and Kurucz (1986), which in turn are based on the unpublished grids of Kurucz. These model colors are a substantial improvement over the ones used previously by Russell, Bessell, and Dopita (1987), which were based on the analysis of Relyea and Kurucz (1978). This is due to the use, in Lester, Gray and Kurucz, of a series of secondary photometric standards for calibration, rather than the single primary standard ( $\alpha$ Lyr) used by Relyea and Kurucz. Unfortunately, the calibration for $m_{1}$ versus $(b-y)$ is still very poor for $(b-y)>0.1$, the region of most interest to us.

Figures $3 a$ and $3 b$ show our intrinsic values (see Table 6) for $c_{1}$ plotted against those for $(b-y)$ for the LMC and the SMC, respectively. On these diagrams 
TABLE 6

Absolute Photometry

\begin{tabular}{|c|c|c|c|c|c|c|c|c|}
\hline $\begin{array}{l}\text { Star } \\
\text { (1) }\end{array}$ & $\begin{array}{l}\text { Spectral } \\
\text { Type } \\
\text { (2) }\end{array}$ & $\begin{array}{l}V \\
(3)\end{array}$ & $\begin{array}{c}E(b-y) \\
(4)\end{array}$ & $\begin{array}{c}(b-y)_{0} \\
(5)\end{array}$ & $\begin{array}{c}\left(m_{1}\right)_{0} \\
(6)\end{array}$ & $\begin{array}{l}\left(c_{1}\right)_{0} \\
(7)\end{array}$ & $\begin{array}{c}T_{\text {eff }} \\
(\mathrm{K}) \\
(8)\end{array}$ & $\begin{array}{c}\log g \\
\text { (phot.) } \\
\text { (9) }\end{array}$ \\
\hline \multicolumn{9}{|c|}{ Galactic } \\
\hline$v \mathrm{Car}^{\mathrm{a}}$ & $\mathrm{A} 8 \mathrm{Ib}$ & 3.15 & 0.157 & 0.04 & 0.126 & 1.373 & 8000 & 1.83 \\
\hline$\alpha \operatorname{Car}$ & F0 Ib-II & -0.73 & 0.000 & 0.110 & 0.128 & 1.512 & 7260 & 1.83 \\
\hline$\alpha$ Lep & F0 Ib & 2.59 & 0.036 & 0.103 & 0.160 & 1.497 & 7330 & 1.97 \\
\hline HR3496 & F2 Iab & 5.74 & 0.261 & 0.133 & 0.140 & 1.484 & 7050 & 1.51 \\
\hline$\delta \mathrm{CMa}$ & F8 Ia & 1.84 & 0.099 & 0.276 & 0.355 & 0.910 & 6250 & 2.23 \\
\hline \multicolumn{9}{|c|}{ SMC } \\
\hline AV 79 & F2 & 13.71 & 0.087 & 0.112 & 0.144 & 1.348 & 7350 & 2.44: \\
\hline AV 107 & F5 & 13.63 & 0.063 & 0.166 & 0.161 & 1.423 & 6770 & 1.50 \\
\hline AV 121 & G0 & 11.31 & 0.110 & 0.334 & 0.373 & 0.931 & 5780 & 1.35 \\
\hline AV 127 & F2 & 12.52 & 0.080 & 0.124 & 0.140 & 1.564 & 7000 & 1.50 \\
\hline AV 198 & F5 & 13.41 & 0.090 & 0.202 & 0.169 & 1.345 & 6520 & 1.32 \\
\hline AV 305 & F5 & 12.86 & 0.014 & 0.164 & 0.143 & 1.492 & 6725 & 1.22 \\
\hline AV 323 & F2 & 13.24 & 0.070 & 0.147 & 0.202 & 1.422 & 6930 & 1.74 \\
\hline AV 369 & G0 & 11.09 & 0.140 & 0.225 & 0.263 & 0.739 & 6680 & 3.41: \\
\hline AV 401 & F2 & 13.20 & 0.065 & 0.087 & 0.140 & 1.626 & 7340 & 1.58 \\
\hline \multicolumn{9}{|c|}{ LMC } \\
\hline G39 & F8 I & 13.22 & 0.090 & 0.264 & 0.257 & 1.070 & 6300 & 1.69 \\
\hline G95 & F0 I & 13.08 & 0.065 & 0.119 & 0.170 & 1.427 & 7230 & 2.08 \\
\hline G104 & F2 I & 13.13 & 0.070 & 0.140 & 0.200 & 1.334 & 7120 & 2.20 \\
\hline G131 & F0 I & 13.30 & 0.070 & 0.099 & 0.188 & 1.455 & 7390 & 2.18 \\
\hline G144 & F0 I & 12.77 & 0.074 & 0.133 & 0.153 & 1.546 & 7000 & 1.50 \\
\hline G231 & F0 I & 12.69 & 0.025 & 0.132 & 0.170 & 1.496 & 7030 & 1.66 \\
\hline G274 & F6: Ia & 12.26 & 0.025 & 0.291 & 0.237 & 0.909 & 6120 & 2.00 \\
\hline G317 & F5 Ia & 11.45 & 0.070 & 0.164 & 0.202 & 1.416 & 6800 & 1.58 \\
\hline G439 & F6 Ia & 11.86 & 0.060 & 0.263 & 0.295 & 1.079 & 6270 & 1.67 \\
\hline G440 & F0: I & 13.17 & 0.090 & 0.128 & 0.157 & 1.561 & 7000 & 1.45 \\
\hline G470 & F0 I & 13.00 & 0.050 & 0.081 & 0.142 & 1.518 & 7500 & 2.11 \\
\hline
\end{tabular}

a The spectral-type was used to determine $T_{\text {eff }}$ and $\log g$ (using the calibration of Johnson 1966), since the published photometry includes a fainter blue companion. 
Figure 3 Plots of the intrinsic $c_{1}$ versus the intrinsic $b-y$ Strömgren indices, overlaid by a model grid of $T_{\text {eff }}$ and $\log g$ (see text) for (a) the SMC, (b) the LMC, and (c) the Galactic stars. 

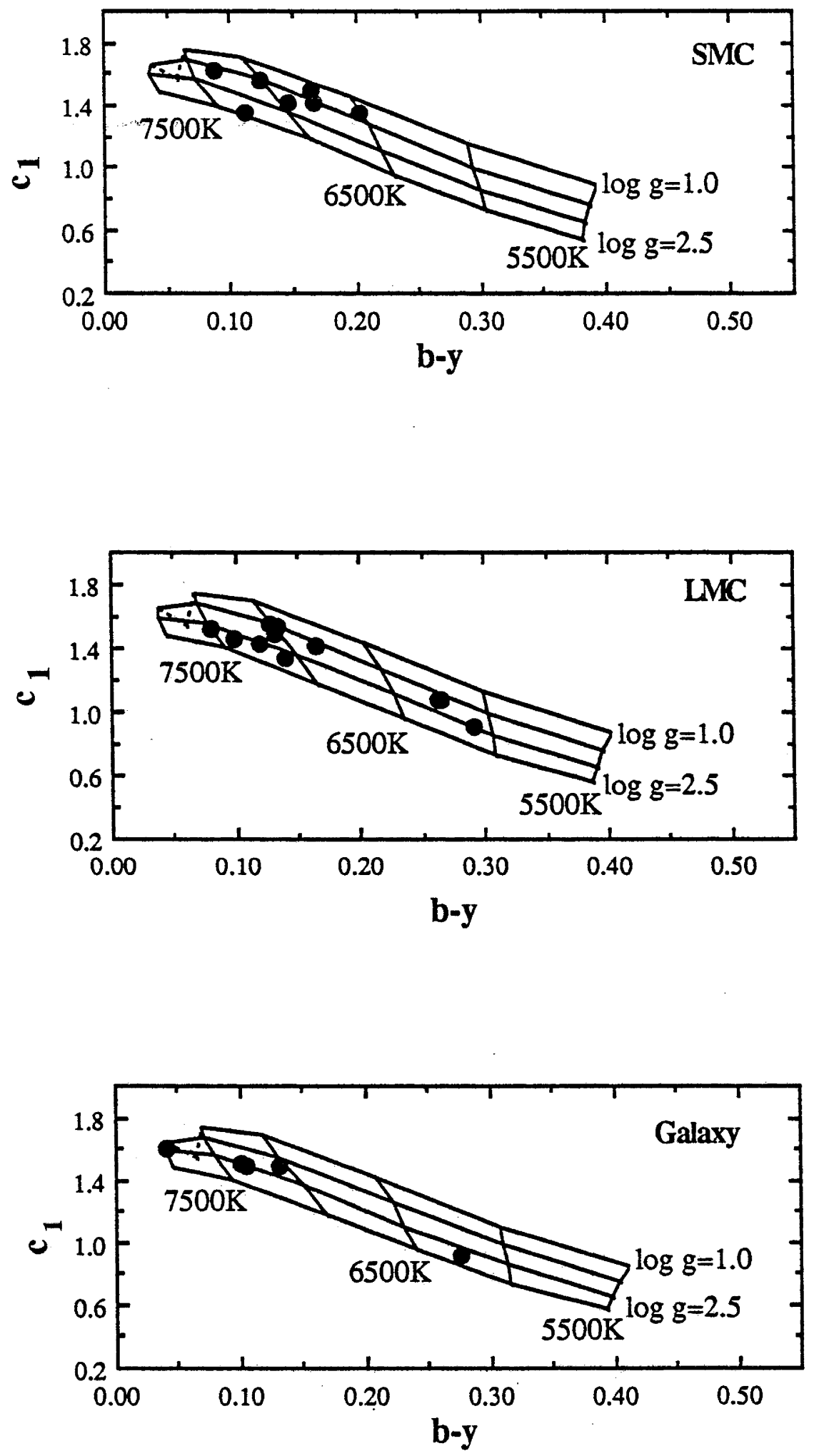
we have overlaid the appropriate model grids interpolated from those of Lester, Gray, and Kurucz (1986). The temperature grid points are spaced at intervals of 500 $\mathrm{K}$, while those of $\log g$ are in steps of 0.5 dex. In addition, we have included for comparison the Galactic standards we used in our abundance analysis, again with the appropriate model of grid points overlaid. We know from an evolutionary point of view (see Fig. 1) the approximate parameter values to expect for each of our stars. As can be seen by comparing these with the results displayed in Figure 3, except for one star (AV 369 as already mentioned) the two sets of parameters appear to agree. Most important, we see that the majority of the Galactic stars are well predicted by the model grid. This means that the calibration of the grids, carried out using less luminous stars, still holds for the types of stars we are considering. The only large error seen for the Galactic stars, is in the gravity of the F8 Ia supergiant $\delta$ CMa. The evolutionary gravity and our spectroscopic analysis of the star both indicate that its gravity is close to $\log g=1.0$ (Luck and Lambert [1981] derive an even lower gravity). The model, however, indicates the same effective temperature for the star as that derived by Luck and Lambert (1981). Thus, with a fine adjustment to the gravity (where necessary) from the spectroscopic analysis of a star, we expect to obtain good measures of both $\log g$ and $T_{\text {eff for our program }}$ stars in the Magellanic Clouds.

Figure 4 illustrates how the calibration for $m_{1}$ versus $b-y$ is still particularly poor for Galactic stars having $b-y>0.15$. When the metallicity is lowered the situation is even worse. As pointed out first by Russell, Bessell, and Dopita (1987), the $m_{1}$ index has ceased to be a good indicator of metallicity for this class of supergiants, and the models in no way match the observations. This is probably due to the effects of the large microturbulent velocities associated with these supergiants, which tend to strengthen the absorption lines and thereby increase the blanketing. 
Figure 4 All the stars in this work plotted on a graph of the intrinsic $m_{1}$ versus the intrinsic $b-y$ Strömgren indices, overlaid by model grids of $T_{\text {eff }}$ and $\log g$ at two different metallicities. 


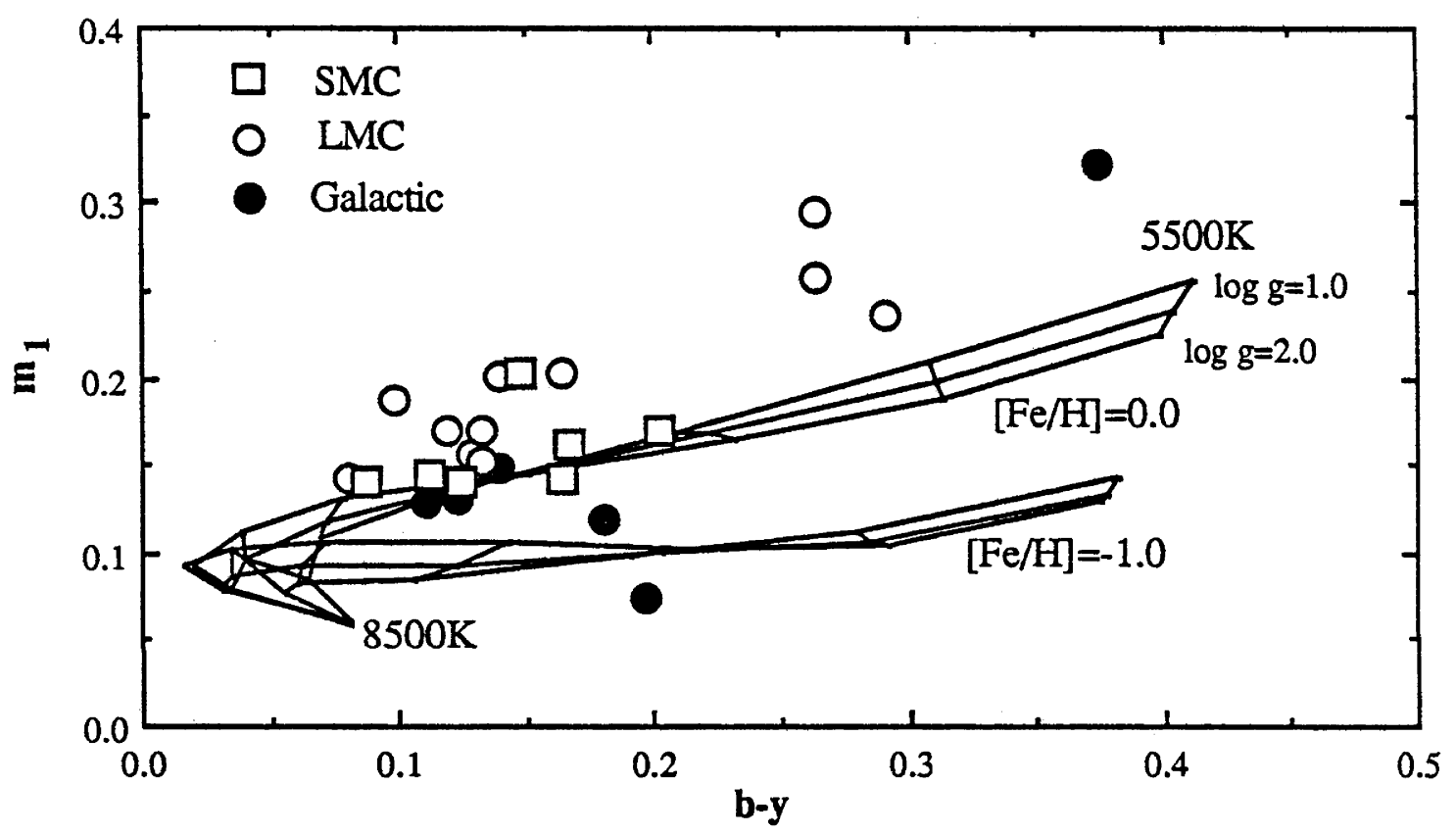




\section{SPECTROSCOPY}

\section{a) The Observations}

Spectroscopic observations were made of all the stars listed in Table 4 except for AV 369 in the SMC and G131, G440, and G470 in the LMC. The observing log for the program stars in the Magellanic Clouds is shown in Table 7. Our aim was to observe as many luminosity class Ib stars as possible (of the order of six stars), using the $3.9 \mathrm{~m}$ Anglo-Australian Telescope (AAT) at Siding Spring in Australia, over a relatively small spectral range (150 - $300 \AA)$ using the IPCS (image photon counting system; see Boksenberg and Burgess 1972) on the RGO spectrograph (with the $82 \mathrm{~cm}$ camera). To supplement this, we planned to observe a few ( perhaps two) somewhat brighter stars (luminosity class Iab - Ia) over a much larger spectral range $(\sim 1000 \mathrm{~A})$ using the echelle spectrograph on the $2.3 \mathrm{~m}$ telescope at Siding Spring, with the PCA (photon counting array (see Stapinski, Rodgers, and Ellis 1981).

As can be seen from Table 7, this plan was successfully carried out in the case of the SMC, where 5 stars were observed on the AAT, and 3 on the $2.3 \mathrm{~m}$. One of the latter (AV 121) was somewhat more luminous than we would have liked for reliable results, but was observed for comparison with the work of Thévenin and Foy (1986). For the LMC, the planned observation of six faint stars on the AAT was defeated by four nights of bad weather. Instead, only three stars were observed on the AAT, but five stars were observed on the $2.3 \mathrm{~m}$ telescope, two of which were of relatively low luminosity. This was possible due to the LMC being 0.5 magnitudes closer to our own galaxy than the SMC.

The majority of stars were observed within the spectral range 4140-5170 $\AA$, as can be seen from Table 7. Only the relatively late star, AV 121 (G0 Ia), and the 
TABLE 7

Spectral Log

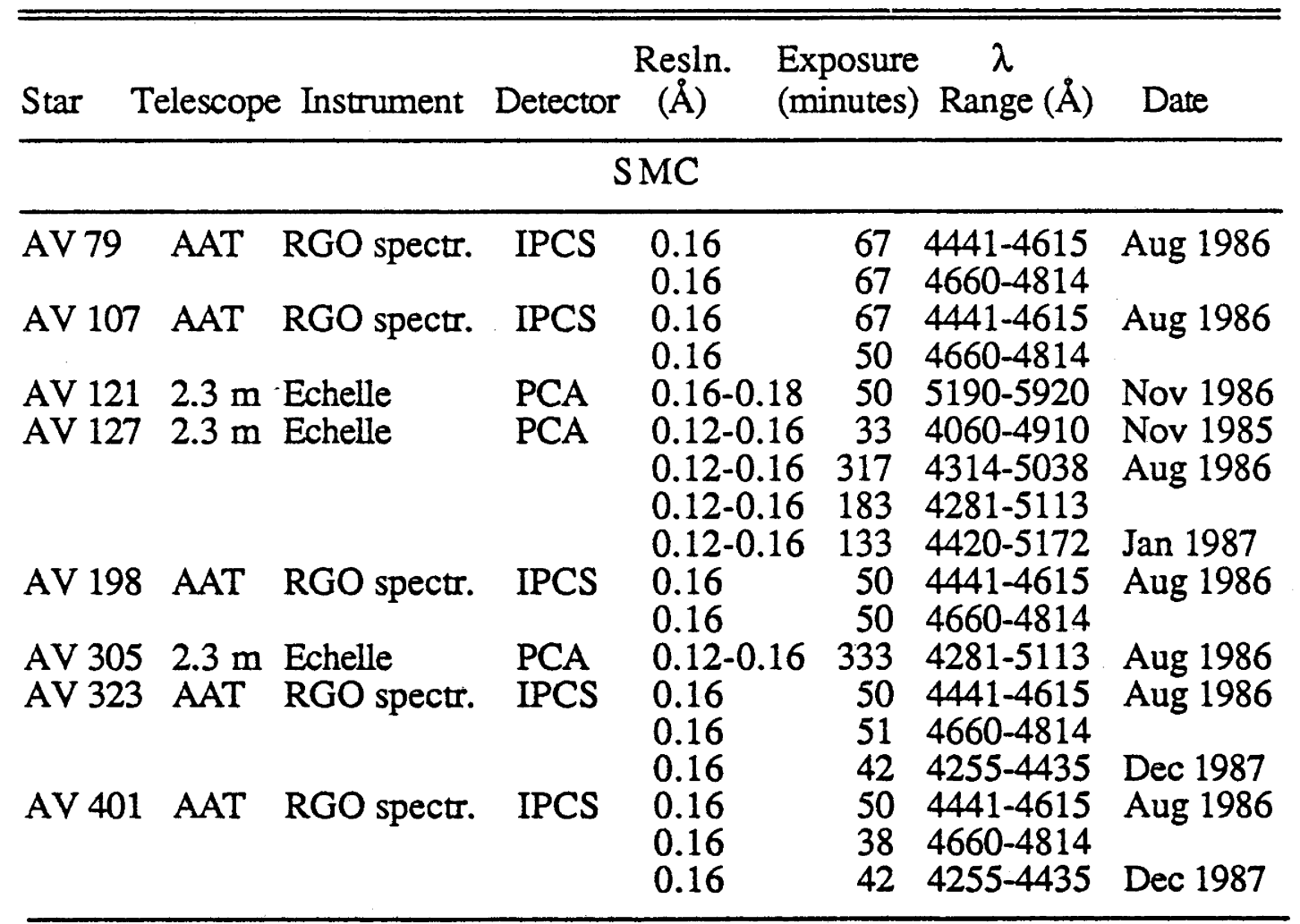

\section{LMC}

\begin{tabular}{|c|c|c|c|c|c|c|c|}
\hline $\begin{array}{l}\text { G39 } \\
\text { G95 }\end{array}$ & $\begin{array}{l}\text { AAT } \\
\text { AAT }\end{array}$ & $\begin{array}{l}\text { RGO spectr. } \\
\text { RGO spectr. }\end{array}$ & $\begin{array}{l}\text { IPCS } \\
\text { IPCS }\end{array}$ & $\begin{array}{l}0.16 \\
0.16 \\
0.16\end{array}$ & $\begin{array}{r}133 \\
32 \\
33\end{array}$ & $\begin{array}{l}4441-4615 \\
4441-4615 \\
4255-4435\end{array}$ & $\begin{array}{l}\text { Oct } 1986 \\
\text { Dec } 1987\end{array}$ \\
\hline G104 & AAT & RGO spectr. & IPCS & & $\begin{array}{l}33 \\
42\end{array}$ & $\begin{array}{l}4441-4615 \\
4255-4435\end{array}$ & Dec 1987 \\
\hline G144 & $2.3 \mathrm{~m}$ & Echelle & PCA & $\begin{array}{l}0.12-0.16 \\
0.12-0.16 \\
0.12-0.16\end{array}$ & $\begin{array}{l}100 \\
100 \\
150\end{array}$ & $\begin{array}{l}4058-4904 \\
4210-4897 \\
4420-5172\end{array}$ & $\begin{array}{l}\text { Jan } 1986 \\
\text { Nov } 1986 \\
\text { Jan } 1987\end{array}$ \\
\hline G231 & $2.3 \mathrm{~m}$ & Echelle & PCA & $\begin{array}{l}-0.16 \\
-0.16 \\
-0.16\end{array}$ & $\begin{array}{r}275 \\
83 \\
100\end{array}$ & $\begin{array}{l}4058-4904 \\
4210-4897 \\
4420-5172\end{array}$ & $\begin{array}{l}\text { Jan } 1986 \\
\text { Nov } 1986 \\
\text { Jan } 1987\end{array}$ \\
\hline G274 & $2.3 \mathrm{~m}$ & Echelle & PCA & 0.16 & $\begin{array}{r}327 \\
50\end{array}$ & $\begin{array}{l}4058-4904 \\
4210-4897\end{array}$ & $\begin{array}{l}\text { Jan } 1986 \\
\text { Nov } 1986\end{array}$ \\
\hline G317 & $2.3 \mathrm{~m}$ & Echelle & PCA & $\begin{array}{r}-0.16 \\
-0.16 \\
-0.16\end{array}$ & $\begin{array}{r}100 \\
50 \\
143\end{array}$ & $\begin{array}{l}4058-4904 \\
4210-4897 \\
4180-4950\end{array}$ & $\begin{array}{l}\text { Jan } 1986 \\
\text { Nov } 1986 \\
\text { Jan } 1987\end{array}$ \\
\hline G439 & $2.3 \mathrm{~m}$ & Echelle & PCA & $\begin{array}{r}-0.16 \\
-0.16 \\
-0.16\end{array}$ & $\begin{array}{r}150 \\
39 \\
75\end{array}$ & $\begin{array}{l}4058-4904 \\
4210-4897 \\
4420-5172\end{array}$ & $\begin{array}{l}\text { Jan } 1986 \\
\text { Nov } 1986 \\
\text { Jan } 1987\end{array}$ \\
\hline
\end{tabular}


standard, $\delta \mathrm{CMa}$, were observed at longer wavelengths (5190-5920 $\AA$ ). The signalto-noise $(\mathrm{S} / \mathrm{N})$ ratios we achieved were extremely variable, from $S / N=15$ to 45 (anything below $\mathrm{S} / \mathrm{N}=15$ was discarded). The dispersions of both the RGO spectrograph at the Cassegrain focus of the AAT and the echelle on the $2.3 \mathrm{~m}$ lay mostly in the range of $4-5 \AA / \mathrm{mm}(0.12-0.16 \AA$ resolution) depending on the precise set-up and the wavelength, but the echelle dispersion was always somewhat higher. Each night of observations included at least one of a Galactic standard to provide a check on the response of the detector. In addition, each of the standards was observed at a very high signal-to-noise ratio $(-150)$ on the 74 inch $(1.88 \mathrm{~m})$ telescope at Mount Stromlo in Canberra, Australia. This is normally difficult for photon counting detectors because of their saturation at count rates greater than $1 / 3$ Hz. It was made possible by smearing the light across many rows of the detector by a fast rotating, hexagonal prism (see Rodgers et al. 1988), placed in front of the slit. The same types of PCA detector and echelle grating were used for the $1.88 \mathrm{~m}$ observations of standards as were used on the $2.3 \mathrm{~m}$ observations.

During the reduction procedure, each star image was divided through by the normalized 2D flatfield taken the night of that observation, then filtered using a three point Hanning window to reduce up-down noise. The sky could then be subtracted in the case of data collected on the RGO spectrograph, but in the case of the echelle data, the orders required rotation to the horizontal, so that they lay parallel to the row direction, before sky (and any interorder light) subtraction could proceed. The data was then placed on a linear wavelength scale by using the Fe lines in the galactic standards, observed at the same setting, for calibration. Finally, the rows containing the stellar image were combined into a one dimensional spectrum, and mildly filtered using a Gaussian filter. After continuum fitting (selecting the line that bisects the noise in regions with no absorption features), the equivalent widths could then be measured using a Gaussian profile fitting routine developed at Mount Stromlo 
Observatory (MSO). The accuracy of the equivalent widths measured in this way was checked using two methods:

a) Comparing the equivalent widths with measurements made using an $\mathrm{Ott}$ planimeter, and

b) Comparing our equivalent widths of the digitized atlas of Procyon (Griffin and Griffin 1979), with those measured by Kato and Sadakane (1982), and by Steffen (1985).

The mean differences between these sets of measurements were as follows:

This work minus planimeter $=-0.1 \pm 8 \mathrm{~m} \AA$,

This work minus Kato and Sadakane $=+3.9 \pm 4 \mathrm{~m} \AA$,

This work minus Steffen $=-0.05 \pm 4 \mathrm{~m} \AA$.

Thus we were confident that the Gaussian fitting routine gave reliable results.

It was found however, that the equivalent widths obtained of standards on the $1.88 \mathrm{~m}$ were somewhat weaker than those measured by Castley and Watson (1980) for the same stars. Figure $5 a$, for instance, shows a comparison between our results for Canopus and those obtained by Castley and Watson. The correlation is, however, very good (see Fig. $5 b$ ), and the slope is repeatable from star to star. The transformation required to achieve agreement between our own data and that of Castley and Watson was simply a constant in the log of $0.17 \pm 0.01$ dex.

Measurements made of the standards Canopus and $\alpha$ Lep, on the $2.3 \mathrm{~m}$ telescope revealed a similar, though slightly smaller, sloped relation in a comparison with Castley and Watson's measurements. This slope was the same as that measured for the star Procyon (F5 IV-V) using the $2.3 \mathrm{~m}$, and compares favourably with measurements made of the digitized atlas of the star (Griffin and Griffin 1979) degraded to the same resolution (see Fig. 6). The repeatability of the relation 
Figure 5 (a) Equivalent widths (in $\mathrm{m} \AA$ ) from this work (RB) obtained on the $1.88 \mathrm{~m}$ telescope with the echelle and the PCA detector, plotted against those reported by Castley and Watson (1980) (CW), for Canopus. The dashed line represents the $45^{\circ}$ line, while the solid line represents the least-squares fit to the distribution.

(b) A comparison between the equivalent widths of Hearnshaw and Desikachary (1982) (HD) with those of Castley and Watson. 

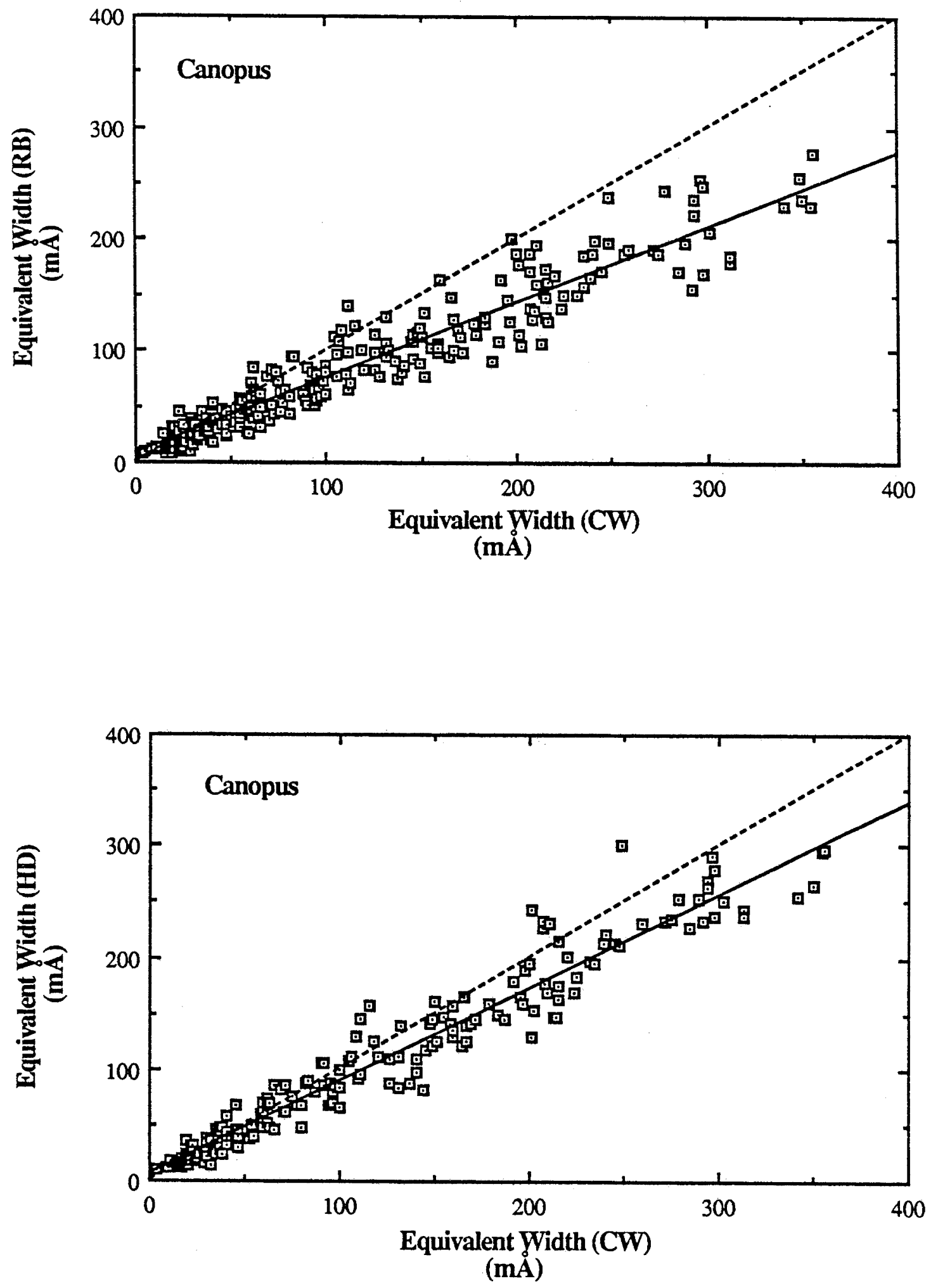
Figure 6 A comparison between equivalent widths of Procyon obtained on the $2.3 \mathrm{~m}$ telescope with the echelle and PCA detector and those derived from a digitized atlas of the same star. The dashed line represents the $45^{\circ}$ line, while the solid line represents the least squares fit to the distribution. 


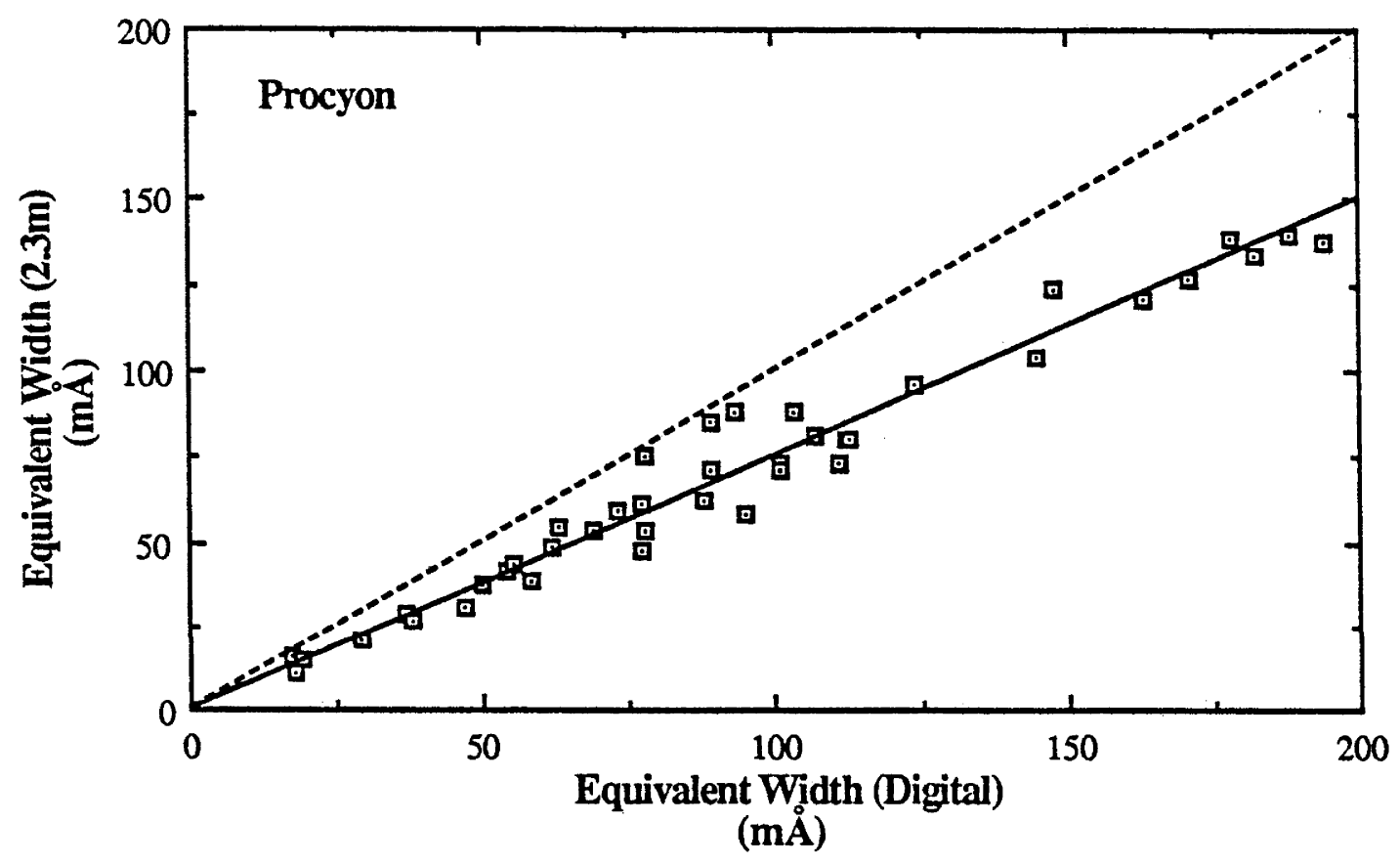


between our results and those of Castley and Watson was also very good from night to night and from star to star, requiring a constant increase in the log of $0.13 \pm 0.02$ dex to achieve agreement.

It may be noted from Figure $5 b$, that Hearnshaw and Desikachary (1982) also measured weaker equivalent widths than Castley and Watson, thus calling into question whether Castley and Watson measured their lines systematically too strong. In addition to our previously mentioned observations of Procyon, the star $\mathfrak{l}$ Car was measured on the $1.88 \mathrm{~m}$ with a $\mathrm{CCD}$ detector and a plane grating (of somewhat lower resolution than the echelle). These results are shown in Figure 7, and it is clear that in this case we had very good agreement with Castley and Watson. We were therefore satisfied that the measurements of Castley and Watson were accurate and, together with the digitized atlas of Procyon, could be used as a satisfactory template for correction of our own results.

Several further tests were made on the PCA-echelle combination on the 1.88 $m$ in an attempt to discover the exact causes for the weak measures of line widths. We concluded that they could not be due to any reasonable form of saturation in the detector, nor could they be due to incomplete subtraction of scattered light (the Balmer lines in Canopus, for instance, were not filled in at the core). Further work to establish the cause of this important problem is still required.

Observations we made using the IPCS on the AAT to observe Procyon agreed very well with the results we obtained from the digitized atlas (see Fig. 8). Therefore, no corrections were required for any observations made using the AAT.

The corrected equivalent widths for the program stars in the Magellanic Clouds and Galactic standards are given in Table 8 in milliangstroms. In this table, any obviously discrepant lines, or those within $12 \AA$ of a hydrogen line, have been discarded. The equivalent widths for lines seen to be slightly blended were 
Figure 7 A comparison between equivalent widths obtained for $\downarrow \mathrm{Car}$ on the $1.88 \mathrm{~m}$ with a plane grating and a CCD detector, and those reported by Castley and Watson (CW) for the same star. The dashed line represents the $45^{\circ}$ line. 


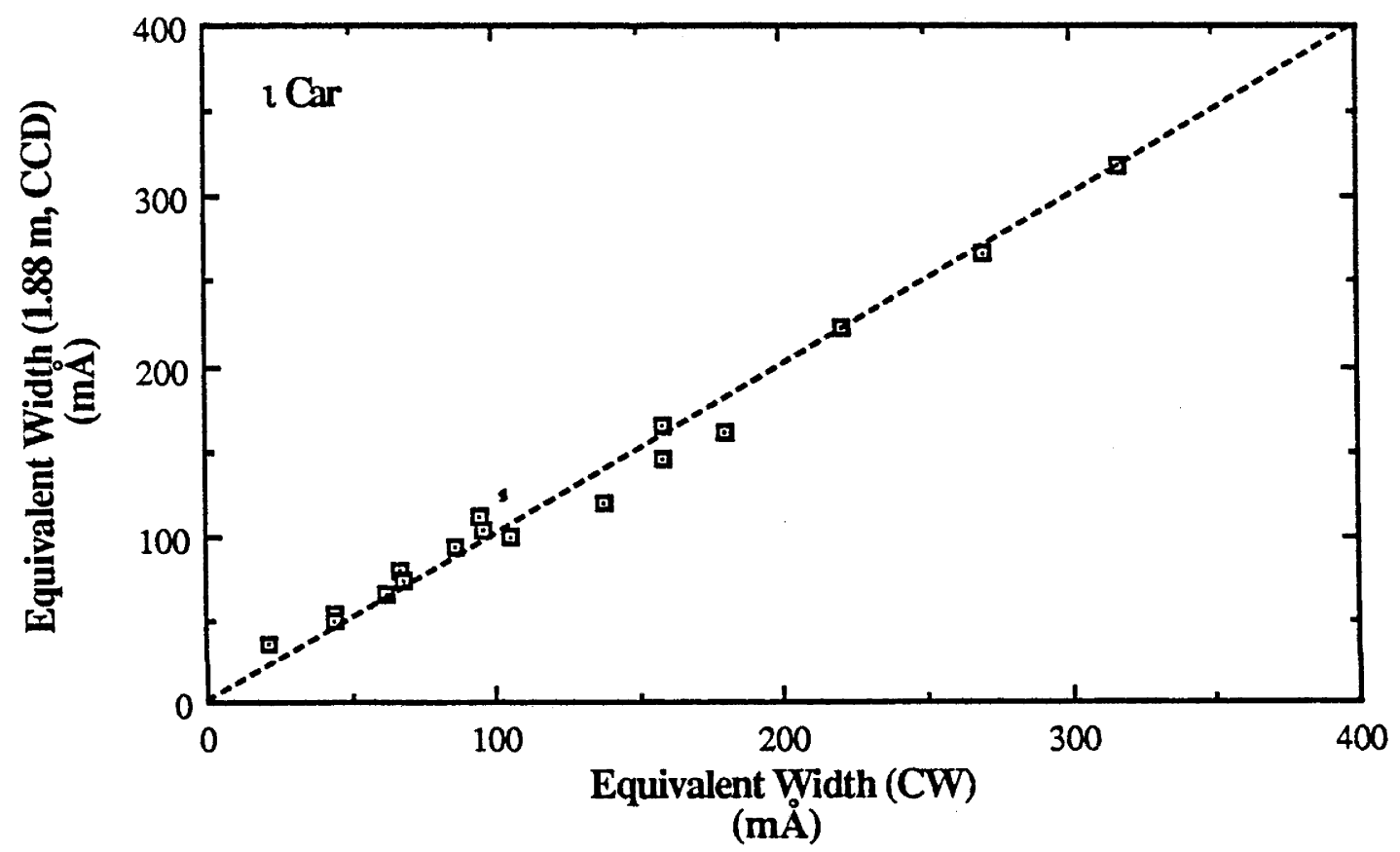


Figure 8 A comparison between equivalent widths obtained with the IPCS on the AAT for Procyon, against those obtained from a digitized atlas (Griffin and Griffin 1979) of the same star. The dashed line represents the $45^{\circ}$ line. 


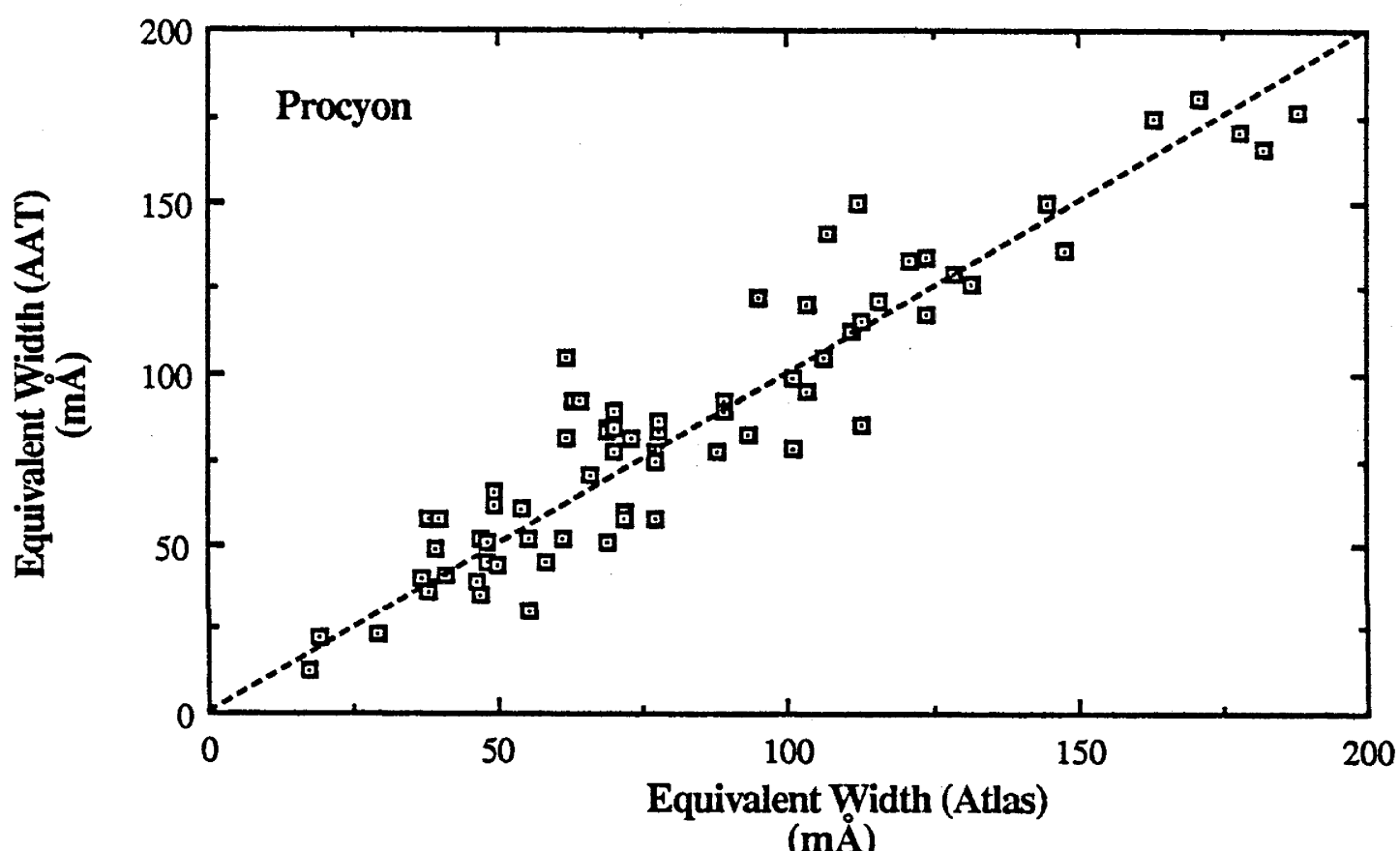


determined empirically for each spectrum individually using the measured relation between the residual depth of the unblended lines and their equivalent widths. The noise level of our observations did not warrant reconstruction of blended line profiles using convolved Gaussians. Although all lines tabulated here have been used in determining the microturbulent velocities $\left(\xi_{T}\right)$, those that were too strong to be used in the abundance analysis have been indicated by italics. Our criterion for deciding if a line was too strong or not was whether an error of $\pm 0.5 \mathrm{~km} \mathrm{~s}^{-1}$ in $\xi_{T}$ (assumed constant with depth in the atmosphere) resulted in an error of 0.1 dex or more in the abundance. Strict observance of these cutoffs, however, was relaxed in cases where it was felt that greater errors would result from discarding a line than from including it. The cutoff for a particular star was determined from Figure 9, which shows our calculations of the change in abundance $(\log \varepsilon)$, for a given equivalent width, due to the indicated $1 \mathrm{~km} \mathrm{~s}^{-1}$ changes in $\xi_{T}$. Interestingly, these relations are almost entirely independent of the effective temperature and gravity over the range of parameters we are interested in. Clearly, for the stars with a higher $\xi_{T}$, the error in abundance resulting from an error in $\xi_{T}$, is much smaller.

\section{b) The Analysis Technique}

In columns (2) and (3) of Table 8, we give both the relative and the absolute oscillator strengths $(\log g f)$ that we used in our abundance analysis, with the references for the absolute strengths given in column (4). We felt that the best method for measuring the abundances for F supergiants in the Magellanic Clouds, was to compare them directly with a well-studied Galactic F supergiant rather than using absolute oscillator strengths or those derived from the solar spectrum. Solar $g f$-values have been used in many studies reported in the literature for stars far removed from the spectral type or metallicity of the Sun (for example; Dickens and 


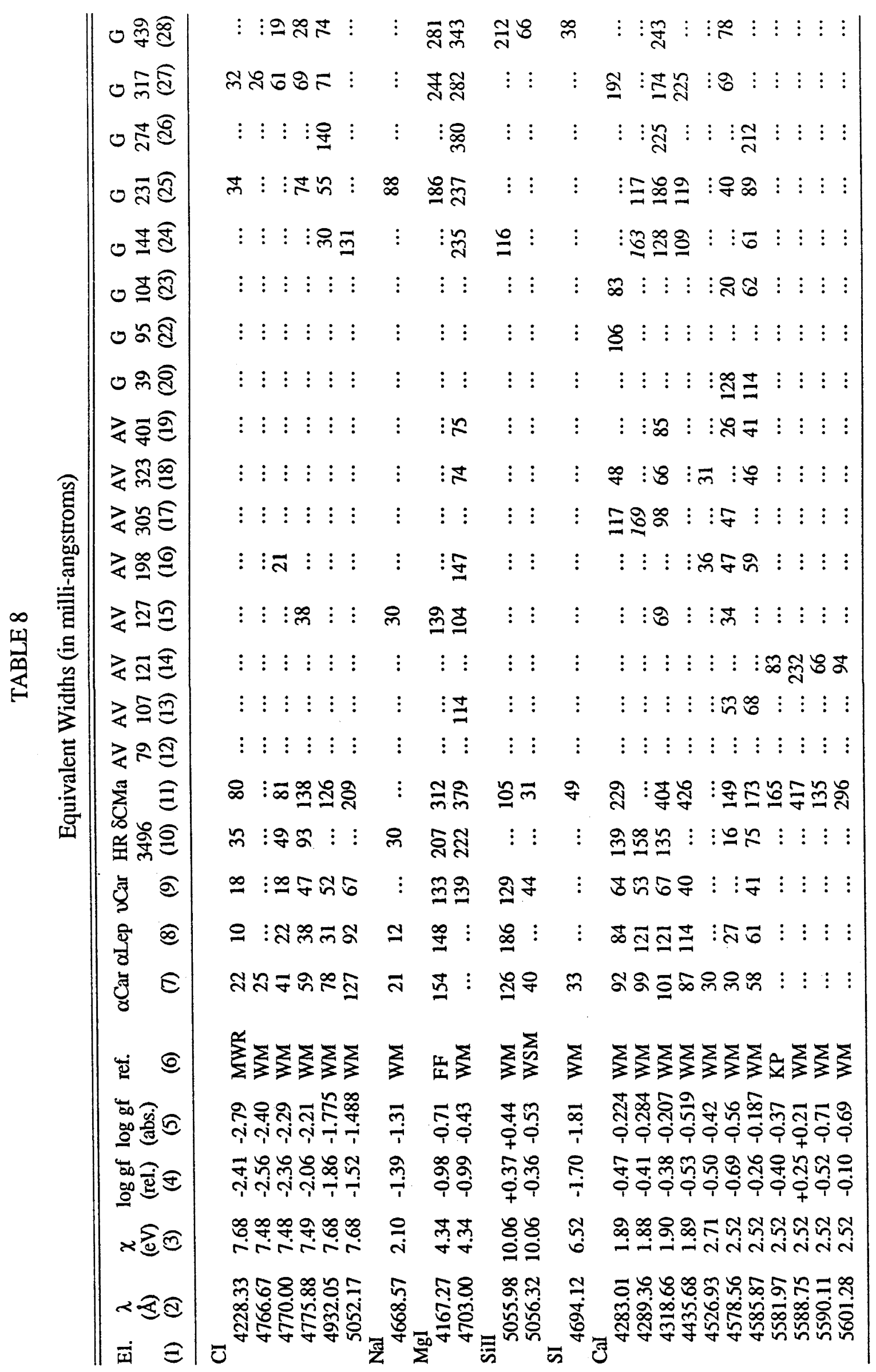




\begin{tabular}{|c|c|c|c|c|}
\hline ৩ & $\vdots$ & 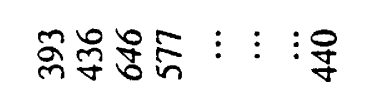 & สี & : : : \\
\hline $0 \stackrel{\bar{m}}{\approx}$ & $\vdots$ & 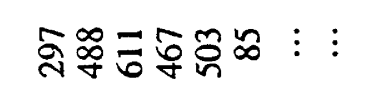 & 2 & @ : \\
\hline ○ 苂氖 & $\vdots$ & 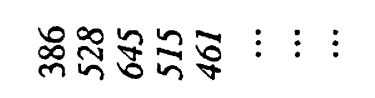 & 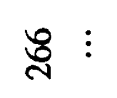 & $\vdots \vdots$ : \\
\hline ৩ & $\vdots$ & ¿: & ถก & 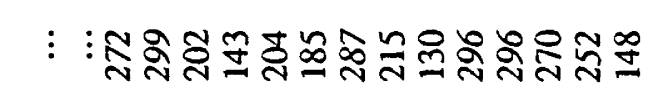 \\
\hline ט 㠻危 & $\vdots$ & 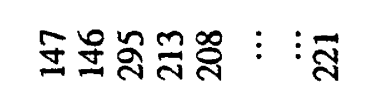 & $89 \vec{q}$ & 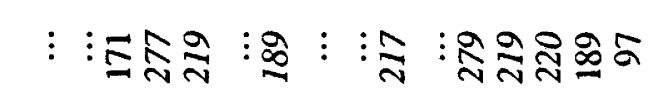 \\
\hline ৩ ఫ을 & $\vdots$ & 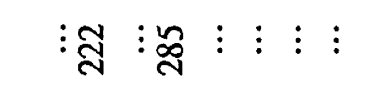 & $\vdots \vdots$ & 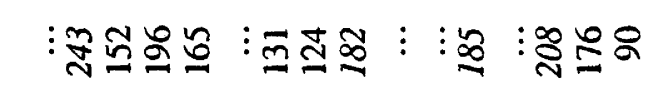 \\
\hline ৩ ๙ส & $\vdots$ & \&萬 $: \stackrel{d}{d}: \vdots \vdots \vdots$ & $\vdots \vdots$ & 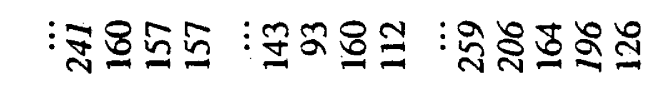 \\
\hline o mిई & $\vdots$ & $\vdots: \vdots: \vdots: \vdots: \vdots: \vdots$ & 芯 & 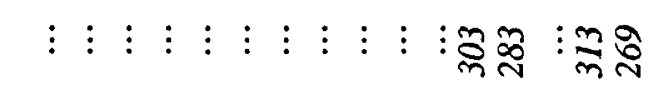 \\
\hline$\gtrless \vec{q}$ & $\vdots$ & 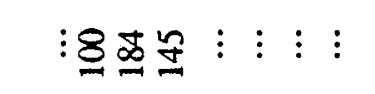 & $\vdots \vdots$ & 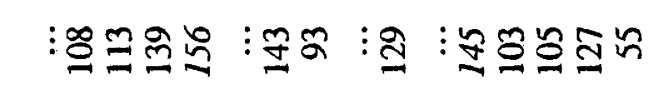 \\
\hline 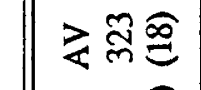 & $\vdots$ & రః్తి $:$ ఫ్సి $\vdots: \vdots \vdots$ & $\stackrel{\infty}{q}:$ & 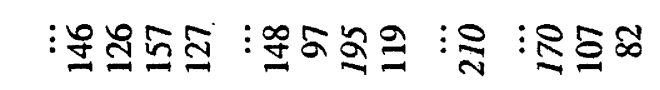 \\
\hline 峞点 & $\vdots$ & 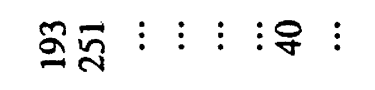 & $\vdots \vdots$ & 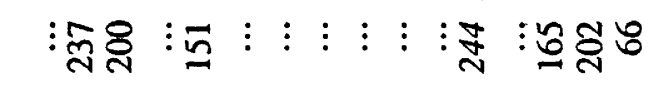 \\
\hline$\gtrless \stackrel{\varrho}{g}$ & $\vdots$ & 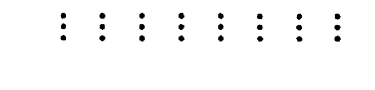 & F⿻ & 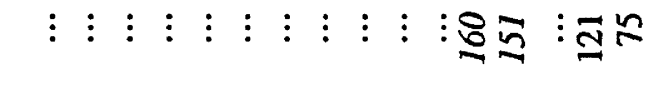 \\
\hline 文氜气 & $\infty$ & 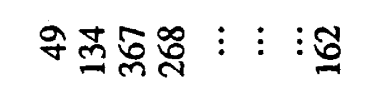 & $\vdots \vdots$ & : \\
\hline$\gtrless \bar{\Xi} \widehat{\Xi}$ & $\vdots$ & $\vdots \quad \vdots \quad \vdots \quad \vdots \quad \vdots \quad \vdots \quad \vdots \quad \vdots$ & $\vdots \vdots$ & 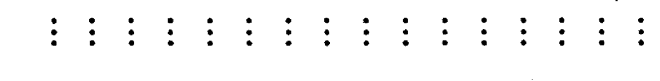 \\
\hline$\gtrless \hat{\theta} \hat{\theta}$ & $\vdots$ & 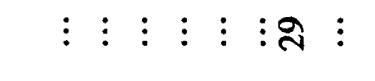 & $F:$ & 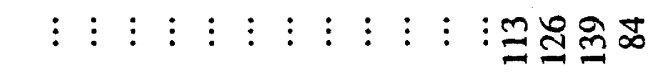 \\
\hline$\gtrless \Omega \widehat{\Xi}$ & $\vdots$ & $\vdots \quad \vdots \quad \vdots \quad \vdots \quad \vdots \quad \vdots \quad \vdots \quad \vdots$ & $\vdots \vdots$ & $\vdots: \vdots \vdots \vdots \vdots \vdots: \vdots \vdots \vdots \vdots \approx 00$ \\
\hline$\sum_{\circlearrowright}^{\pi} \cong$ & $\vdots$ & 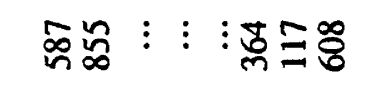 & $\stackrel{\mathscr{N}}{:}:$ & 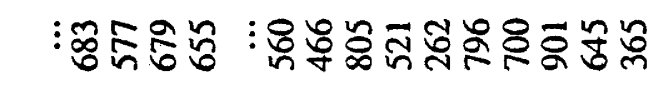 \\
\hline 必㐫司 & $\vdots$ & 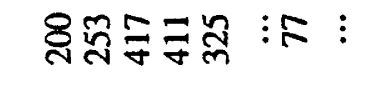 & 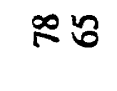 & 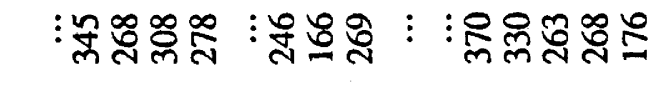 \\
\hline ฮูँ & $\vec{m}$ & 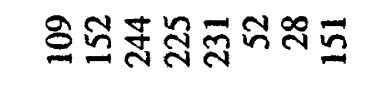 & $\vdots \grave{\text { ก }}$ & 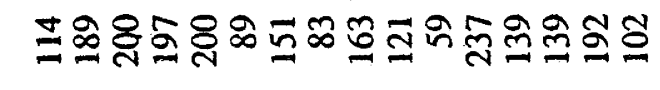 \\
\hline क्षे & $F$ & 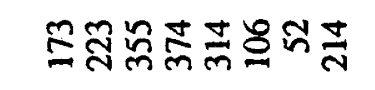 & t & 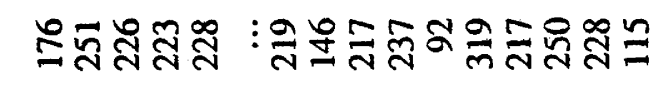 \\
\hline 政 & 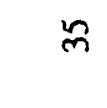 & 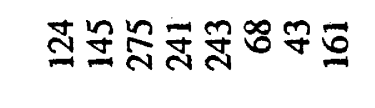 & 50 & 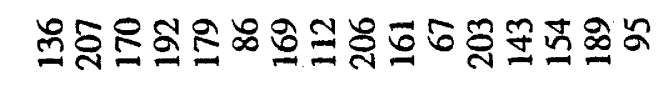 \\
\hline$\dot{\Phi}$ & 운. & 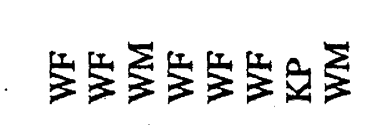 & $\sum_{n=1}^{\infty}$ & $3 \sum_{3}$ \\
\hline 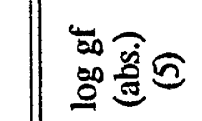 & $\stackrel{1}{\pi}$ & 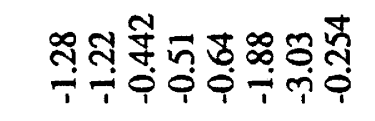 & 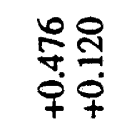 & 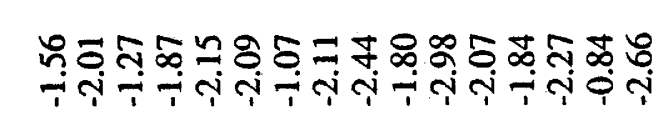 \\
\hline 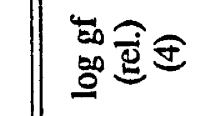 & $\hat{\grave{\varphi}}$ & 꾸ำ & ำ่ำ & 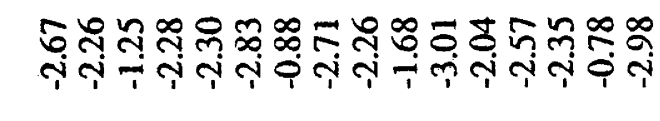 \\
\hline ×矛同 & $\stackrel{n}{n}$ & 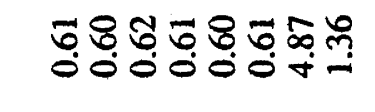 & 象象 & 車承 \\
\hline 7 & ت & 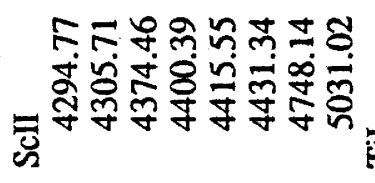 & กิธี丶 & 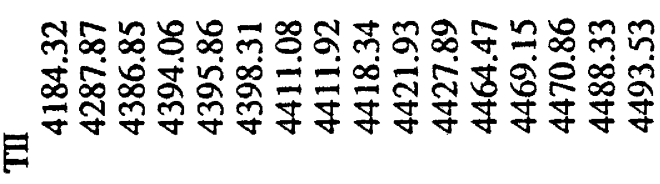 \\
\hline
\end{tabular}




\begin{tabular}{|c|c|c|c|c|}
\hline ৩ $\stackrel{\vec{\gamma}}{\vec{d}}$ & 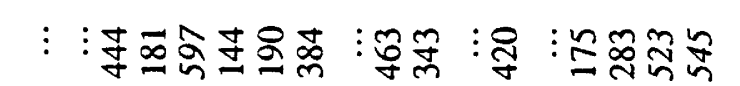 & $: m$ & $\frac{\sigma}{m}:$ & :ले \\
\hline 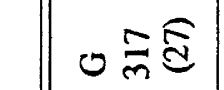 & 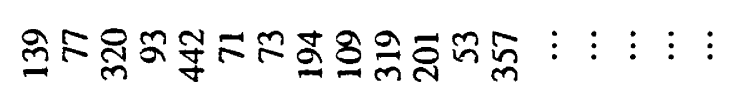 & $\vdots \vdots \vdots$ & 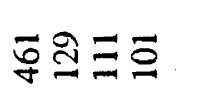 & $\sqrt[n]{n} \infty$ \\
\hline ৩ $\underset{ }{\overparen{d}}$ & 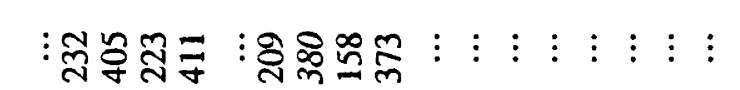 & $\vdots \vdots \vdots$ & $\vdots \underset{N}{2} \stackrel{2}{2}$ & $\vdots \vdots \stackrel{\text { ก }}{\Upsilon}$ \\
\hline ৩ & 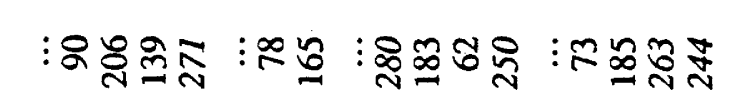 & $\vdots \check{m} \bar{n}$ & $\vdots \widehat{6}_{\infty}^{\infty} \vdots$ & $\vdots: \mathbb{Z}$ \\
\hline & 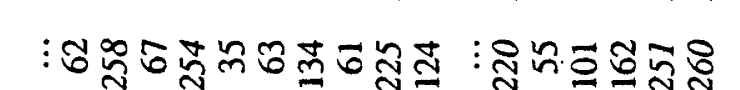 & $n \vdots$ & $\vdots \vdots \vdots \vdots$ & : \\
\hline o 节 & 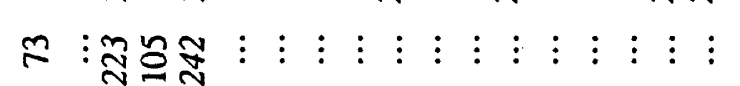 & $\vdots \vdots \vdots$ & $\vdots \vdots \quad \vdots \quad \vdots$ & $\vdots \vdots$ in \\
\hline $0 \curvearrowleft$ & $\vdots$ ด̊ษฎ $\vdots \vdots \vdots \vdots: \vdots \vdots \vdots \vdots \vdots \vdots \vdots \vdots$ & $\vdots \vdots \vdots$ & $\vdots \vdots \vdots \vdots$ & $\vdots \vdots$ \\
\hline o & 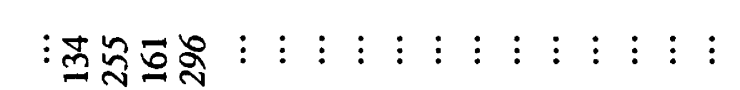 & $\vdots \vdots \vdots$ & $\vdots \vdots \vdots \vdots$ & $\vdots \vdots$ : \\
\hline$\gtrless \bar{\sigma}$ & 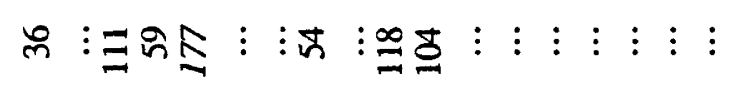 & $\vdots \quad \vdots \quad \vdots$ & $\vdots: \vdots: \vdots$ & $\vdots:$ : \\
\hline$\gtrless \tilde{n}^{2}$ & 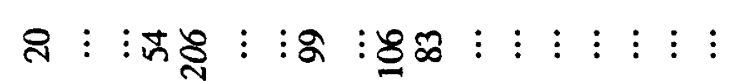 & $\bar{\sim}: \vdots$ & $\vdots \vdots \vdots \vdots$ & $\vdots \vdots \curvearrowleft$ \\
\hline ये & 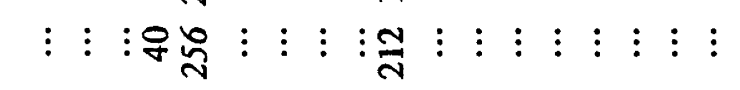 & $\vdots \vdots \vdots$ & $\vdots: \vdots \vdots$ & 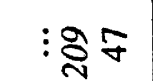 \\
\hline 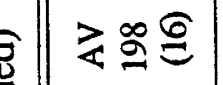 & 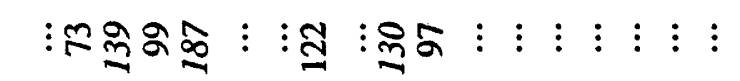 & $\vdots \vdots \overline{\mathrm{N}}$ & $\vdots: \vdots \vdots$ & $\vdots: \infty$ \\
\hline$\gtrless \bar{\Xi}$ & $\vdots$ :qす) & $\vdots \vdots \vdots$ & $\vdots: \vdots \vdots$ & : శ্ণু \\
\hline$\gtrless \bar{\Xi}$ & 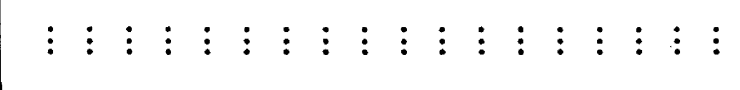 & $\vdots \vdots \vdots$ & $\vdots: \vdots \vdots$ & $\vdots:$ \\
\hline$\gtrless \hat{g} \hat{g}$ & 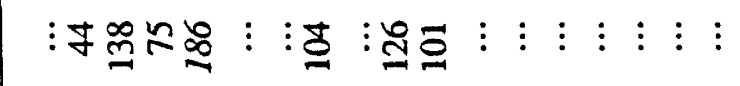 & $\vdots \vdots \cong$ & $\vdots \vdots \vdots \vdots$ & $\vdots: \overline{7}$ \\
\hline 玄ล气 & 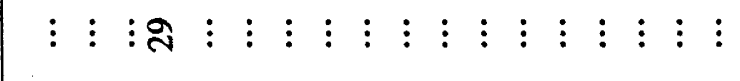 & $\vdots \vdots \vdots$ & $\vdots \vdots \vdots \vdots$ & $\vdots$ \\
\hline$\sum_{0}^{\pi} \cong$ & 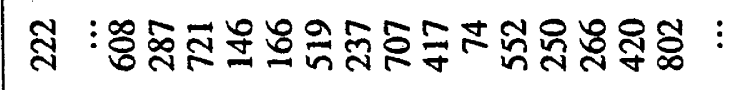 & 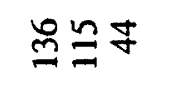 & $\vdots: \cong !$ & $\vdots:$ 芦 \\
\hline 茫高总 & 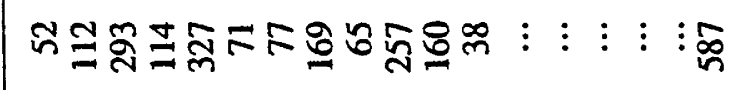 & $\vdots$ ก & ) & ! ஜ্রুঃ \\
\hline ఫ્ર & 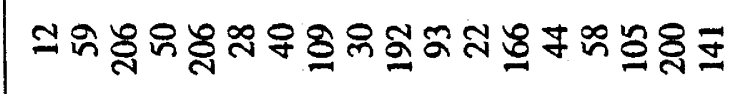 & $\vdots \vdots \vdots$ & กี่ะ & $\exists \mathcal{Z}$ \\
\hline$\widehat{0}$ & 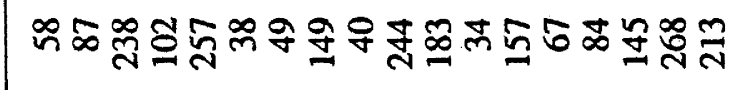 & 프 & :\&\& & ㅇํㅇ \\
\hline$E$ & 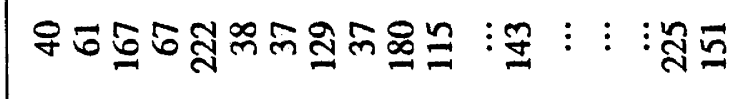 & $\infty$ & ฐరరా & mミڤ \\
\hline$\ddot{\Xi} \theta$ & 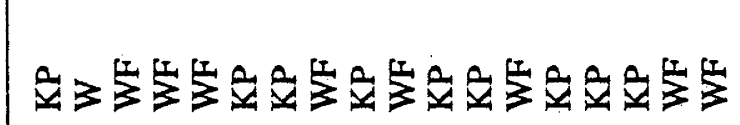 & 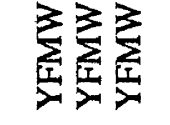 & 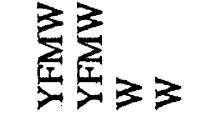 & 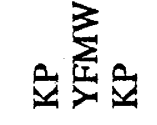 \\
\hline 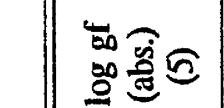 & 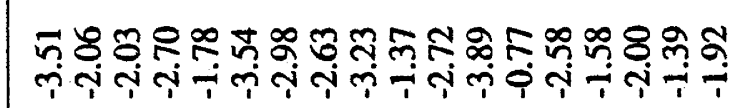 & 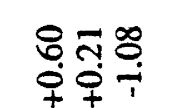 & ตุ่ง & \\
\hline & 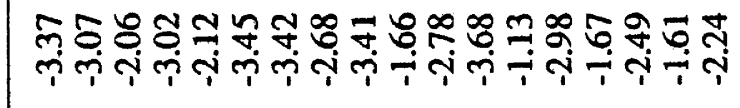 & 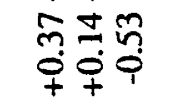 & 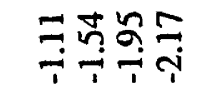 & 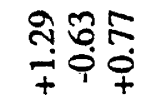 \\
\hline & 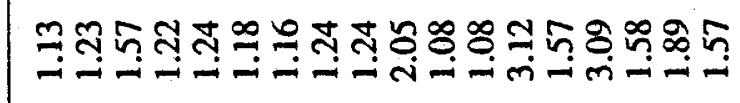 & ్ㅠㅇㅛ & ำดำ & $\therefore 8:$ \\
\hline & $\begin{array}{l} \pm \\
8 \\
8\end{array}$ & 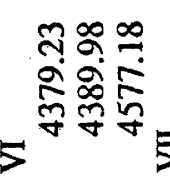 & तิં & ô \\
\hline
\end{tabular}




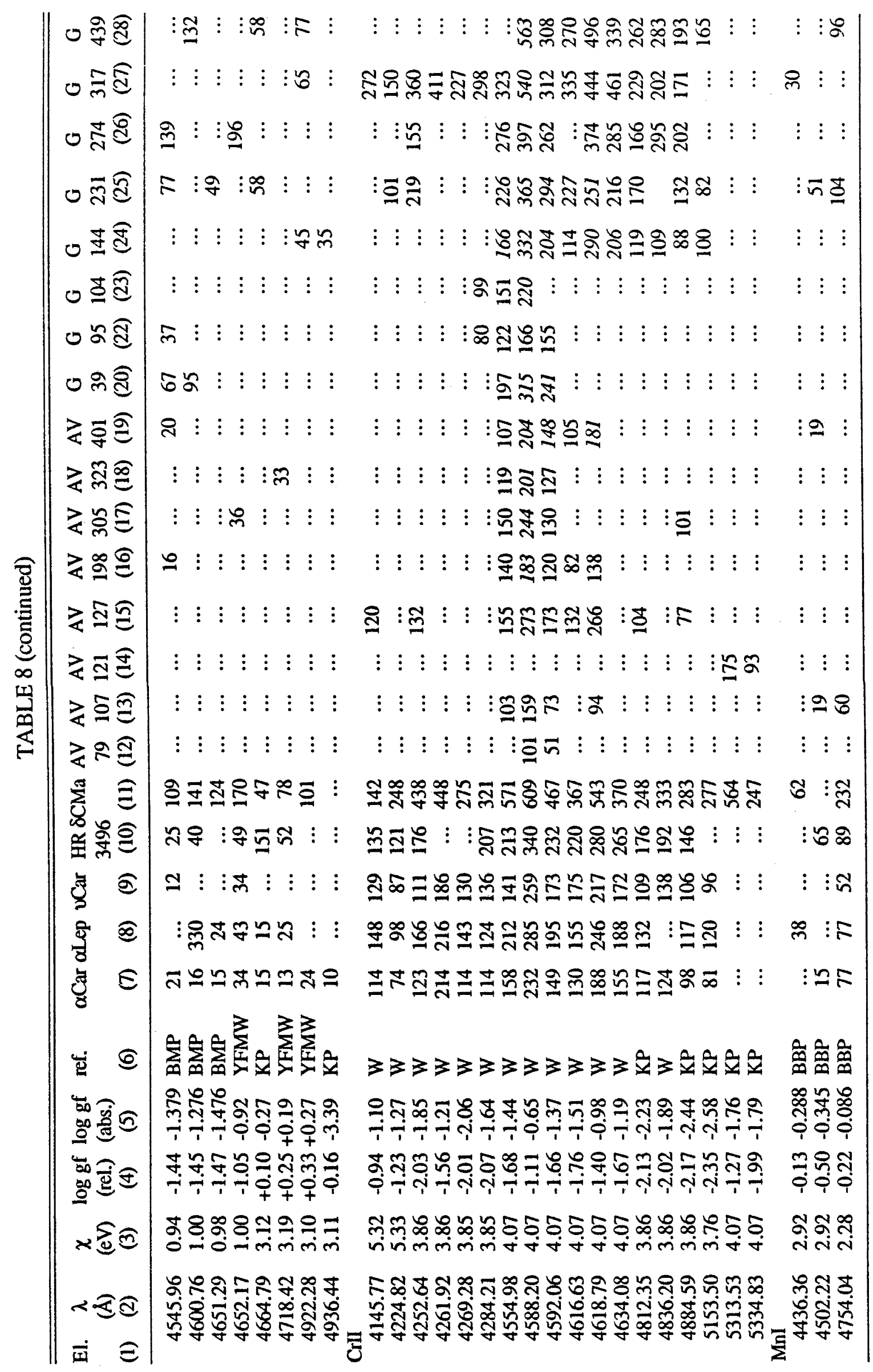




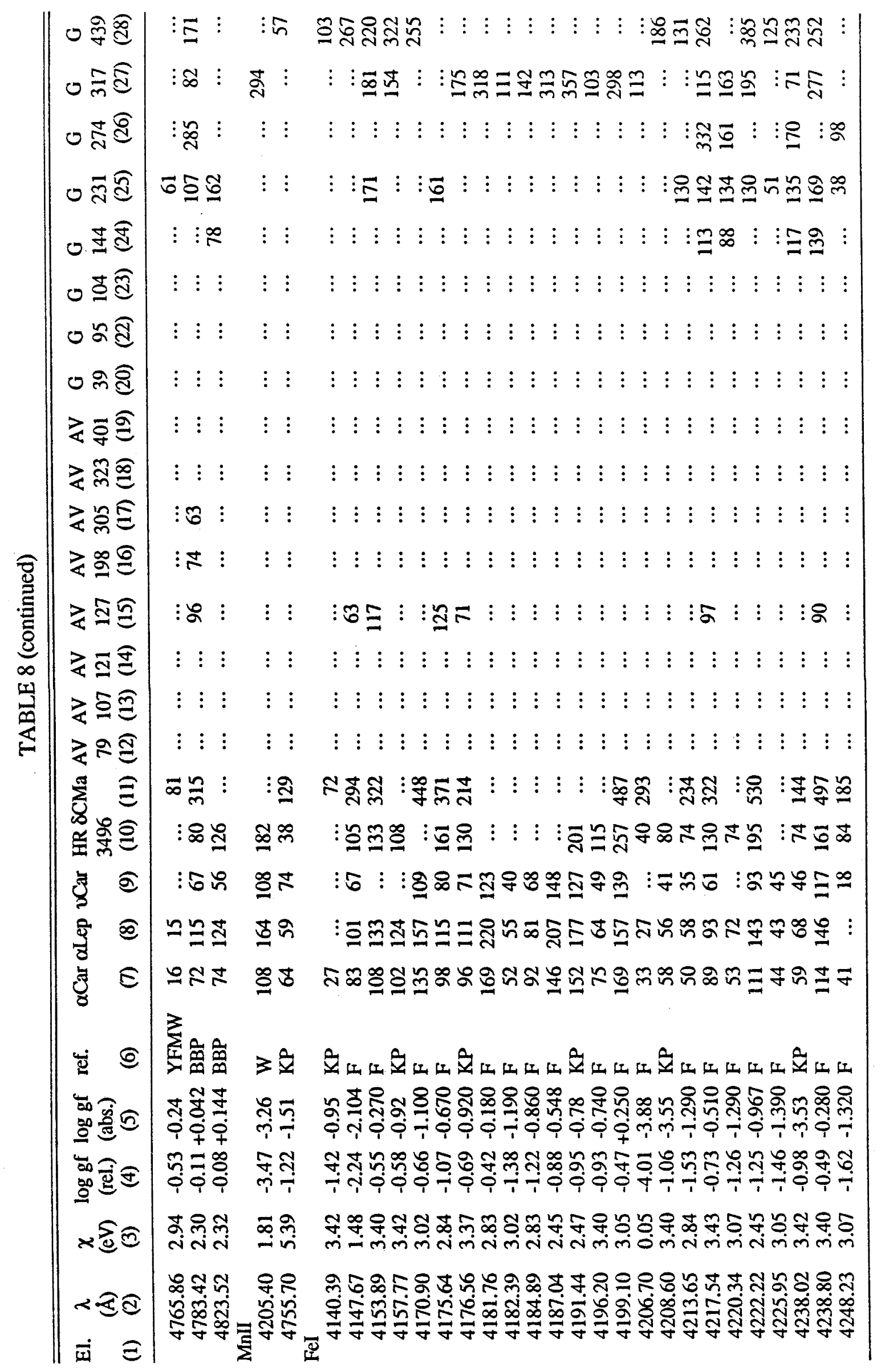




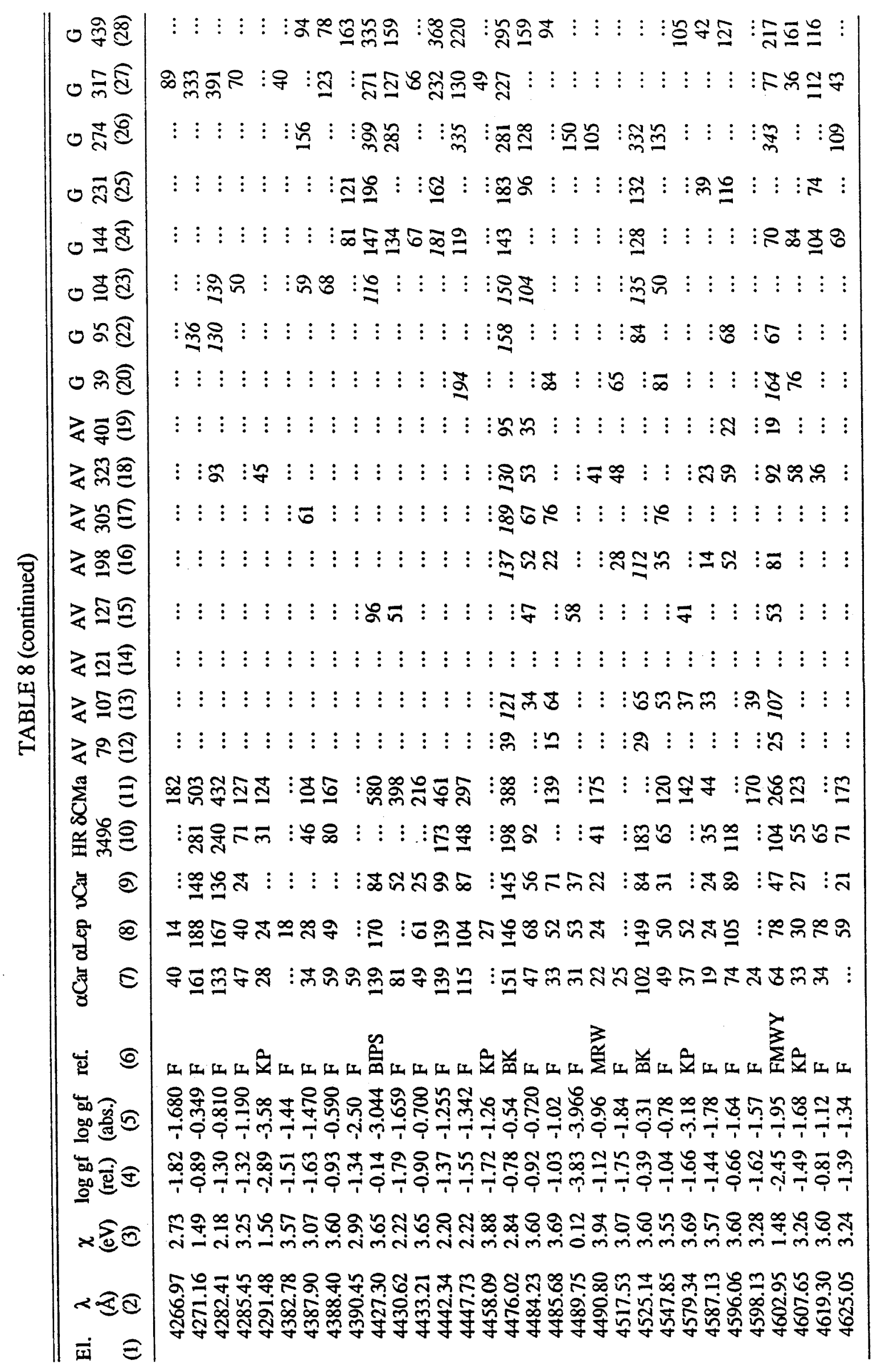




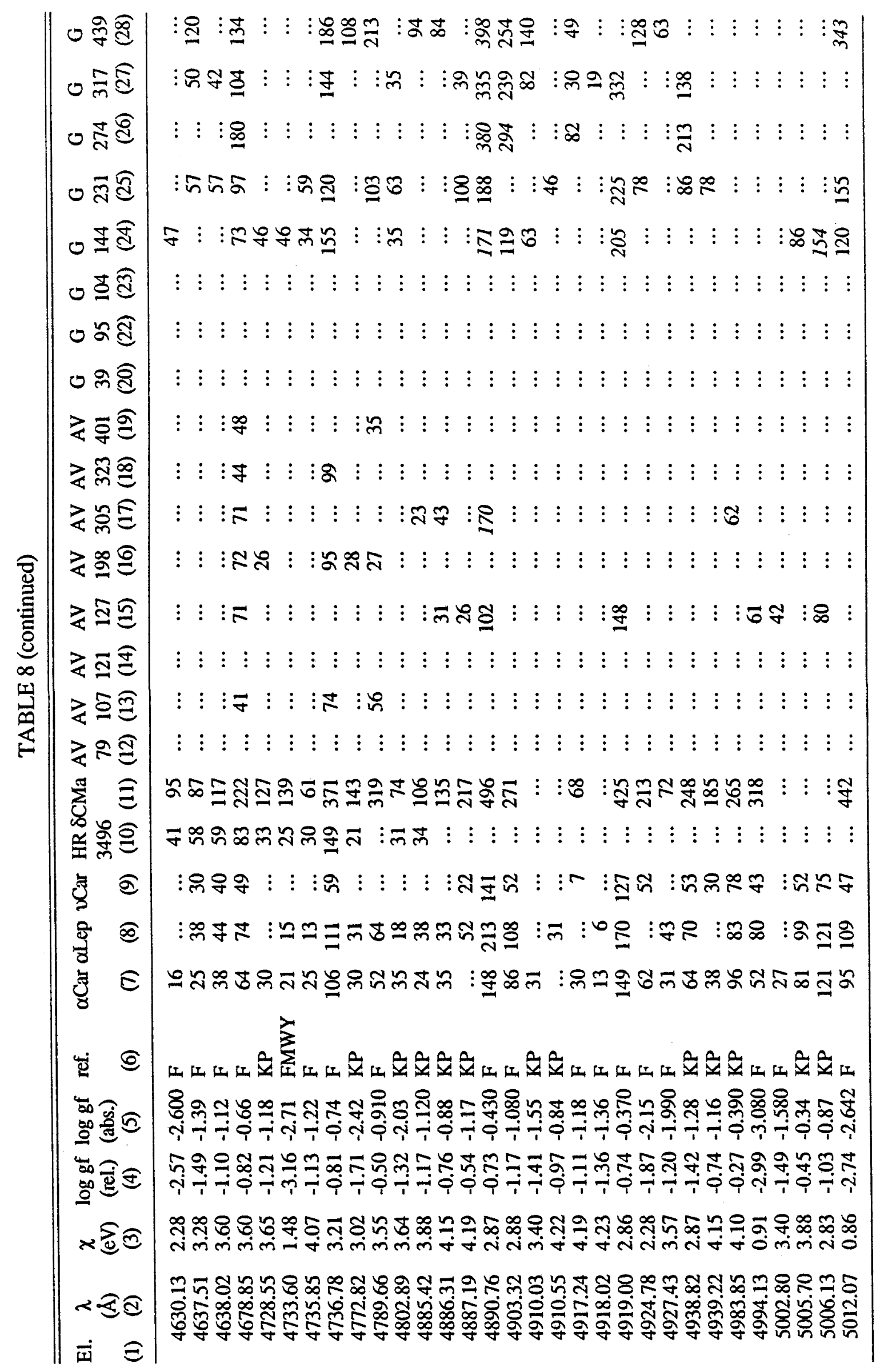




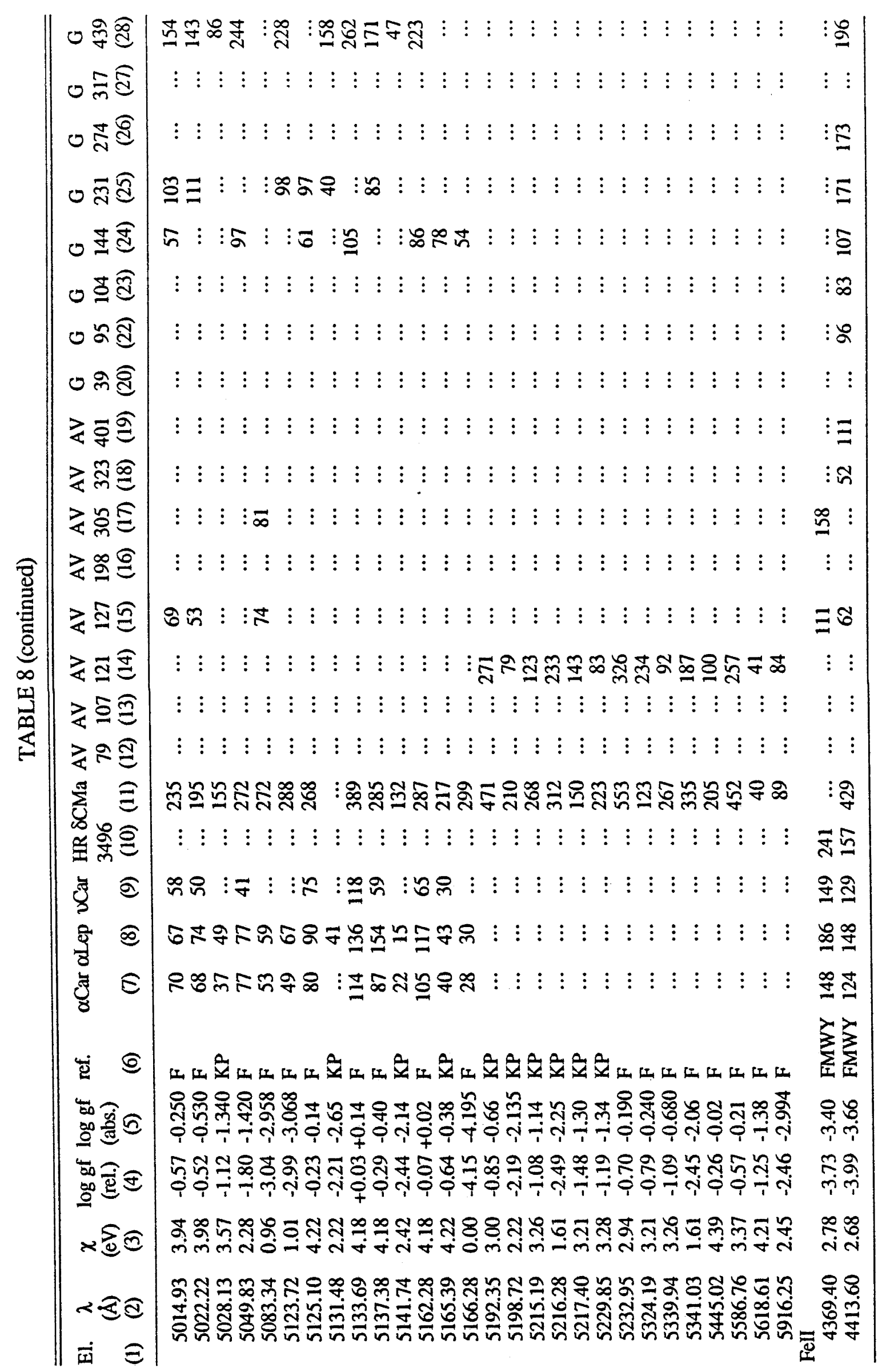




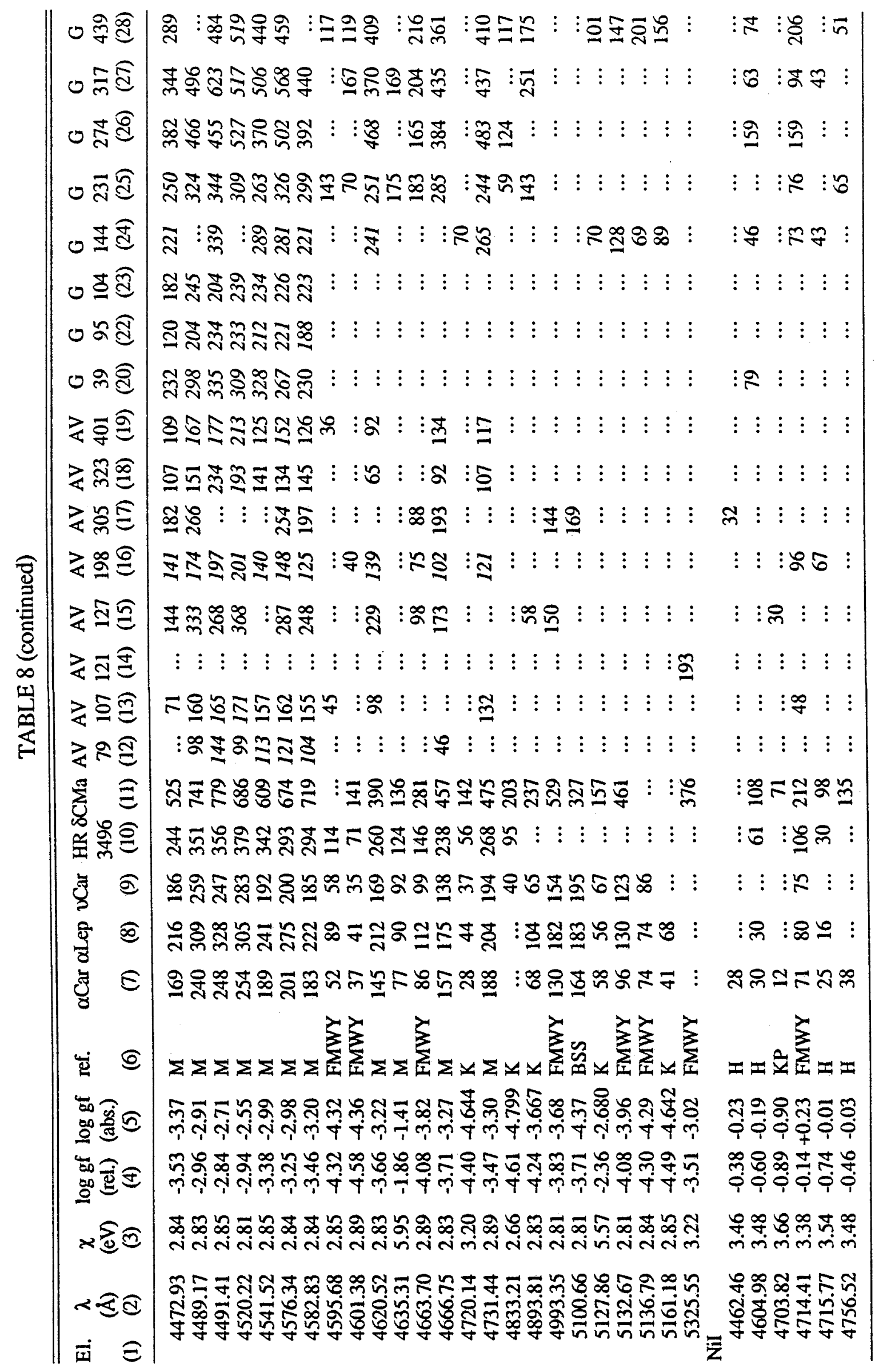




\begin{tabular}{|c|c|c|c|c|c|c|c|}
\hline 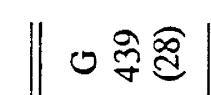 & 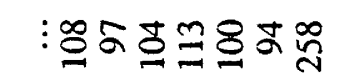 & $\vdots$ & & 6 & 2 & & 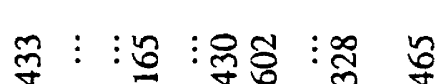 \\
\hline o $) \widehat{m}$ & $\mathcal{F} \bar{n}: \vdots: \vdots \vdots \vdots$ & a & $\vdots$ & $\vdots$ & ลे & & 总 $:$ : \\
\hline 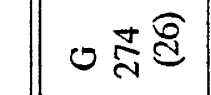 & $\vdots: \nsubseteq:: \vdots:: \vdots:$ & $\cong$ & $\vdots$ & $\vdots:$ & ః & $\vdots$ & 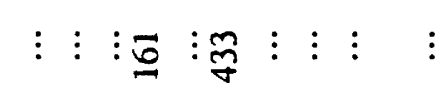 \\
\hline 0 & $\vdots \vdots: \vdots: \mathbb{N}:$ & $\vdots$ & $\vdots$ & $\bar{a}$ & 莴 & & సి \\
\hline & 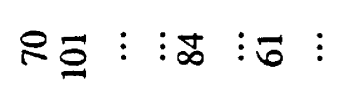 & $\vdots$ & $\vdots$ & $\vdots 8$ & $\$$ & & $\mathscr{\infty}::::::_{\infty}^{\infty} \underset{Q}{\infty}:{ }^{\infty}$ \\
\hline & : $: \vdots: \vdots: \vdots: \vdots: \vdots$ & $\vdots$ & $\vdots$ & $::$ & $\vdots$ & \pm & $\infty$ \\
\hline & $\vdots: \vdots: \vdots: \vdots: \quad: \quad: \quad \vdots$ & $\vdots$ & $\vdots$ & $::$ & $:$ & $\vdots$ & 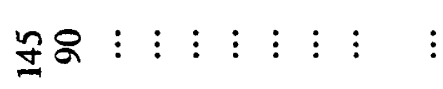 \\
\hline o & $: \vdots: \quad: \quad: \quad \vdots \quad: \quad: \quad \vdots$ & $\vdots$ & $\vdots$ & $::$ & $\vdots$ & कू & 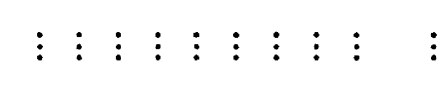 \\
\hline & $:: \vdots: \vdots: \vdots: \vdots: \vdots$ & $\vdots$ & $\vdots$ & $: \vdots$ & $\vdots$ & F & 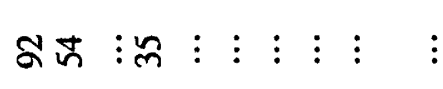 \\
\hline & $:: \vdots: \vdots: \vdots: \vdots: \vdots$ & $\vdots$ & $\vdots$ & $\vdots:$ & $\vdots$ & $\vdots$ & Oํ \\
\hline$r$ & $\vdots: \vdots: \vdots: \vdots \quad \vdots \quad: \quad \vdots$ & $\vdots$ & $\bar{\lambda}$ & $: \vdots$ & $\vdots$ & $\overline{6}$ & 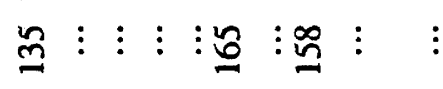 \\
\hline 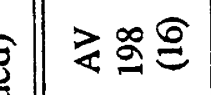 & $:::::::::: \vdots$ & $\vdots$ & $\vdots$ & $::$ & $\vdots$ & i & $:: \vdots:: \vdots: \vdots: \vdots: \vdots$ \\
\hline$<$ & 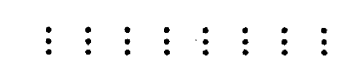 & 8 & g & $\vdots: \vdots$ & 芦 & $\vdots$ & 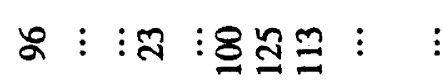 \\
\hline $\mathbb{Q} \bar{\Xi}$ & 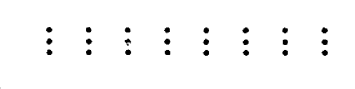 & $\vdots$ & $\vdots$ & $::$ & $\vdots$ & $\vdots$ & $:: \vdots:: \vdots:: \vdots: \vdots: \vdots:$ \\
\hline$<$ & : : : : : : : : : : : & $\vdots$ & $\vdots$ & $: \vdots$ & $\vdots$ & in & : : : : : : : : $: \vdots$ \\
\hline & 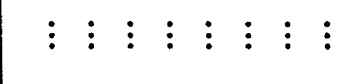 & $\vdots$ & $\vdots$ & $: \vdots$ & $\vdots$ & $\vdots$ & $::: \vdots: \vdots$ \\
\hline & 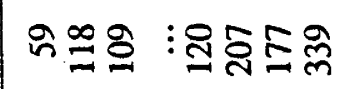 & $\cong$ & $\stackrel{8}{\circ}$ & ะะ్ & $\vdots$ & $\infty$ & 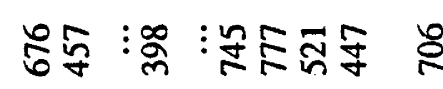 \\
\hline & F\& & f & $\vdots$ & $\infty$ & 鿄 & $\vdots$ & 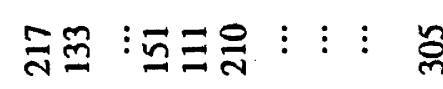 \\
\hline ఫ్ & 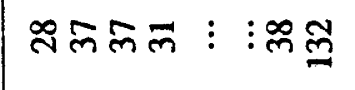 & $\stackrel{\sim}{\infty}$ & $\vdots$ & $\vdots れ$ & 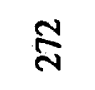 & $\vdots$ & 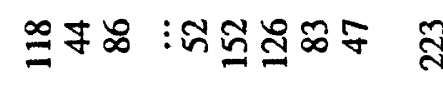 \\
\hline$\widehat{\Xi}$ & 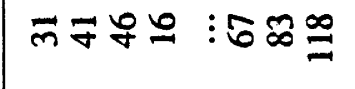 & F & $\vdots$ & $\approx \mathcal{7}$ & $\stackrel{t}{n}$ & $\vdots$ & 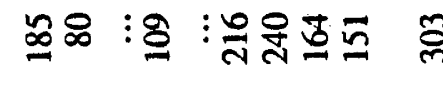 \\
\hline 灵 $\varepsilon$ & 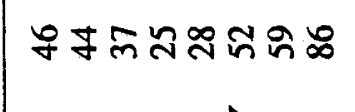 & $\approx$ & $\vdots$ & 要 & $\underset{\infty}{\infty}$ & \& & 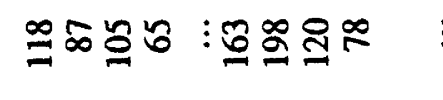 \\
\hline 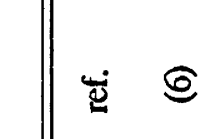 & 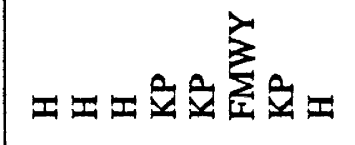 & 3 & $\sum$ & $\sum_{3}^{2}$ & $\sum$ & $\hat{y}$ & 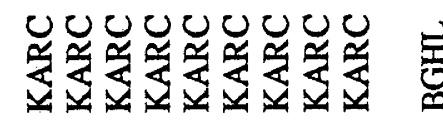 \\
\hline & 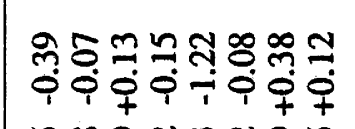 & ڤ્ণ & $\overline{5}$ & 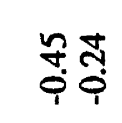 & $\frac{8}{9}$ & $\hat{n}$ & 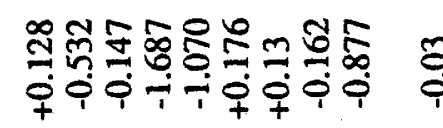 \\
\hline & 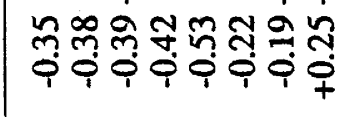 & ָூ & $\stackrel{8}{n}$ & 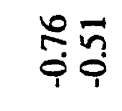 & 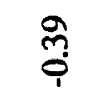 & J & 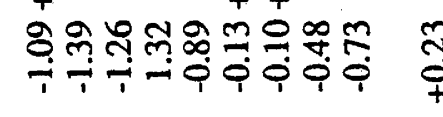 \\
\hline & 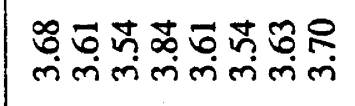 & $\stackrel{\Re}{\stackrel{\leftrightarrow}{+}}$ & $\stackrel{a}{g}$ & 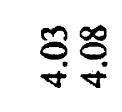 & 8 & $\infty$ & 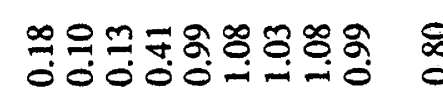 \\
\hline & 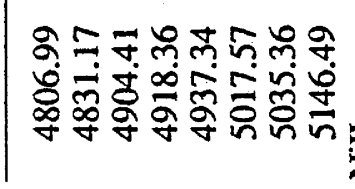 & 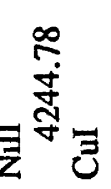 & & $\underline{x}$ & & & 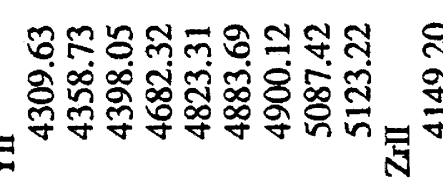 \\
\hline
\end{tabular}




\begin{tabular}{|c|c|c|c|c|c|}
\hline ৩ ঞ্ণি & 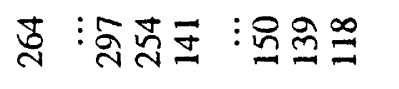 & 80 & a & ల్ల & - \\
\hline o & 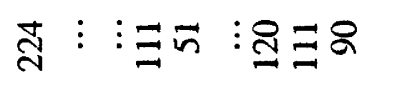 & 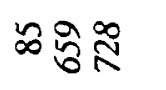 & $:: \vdots: 2$ & 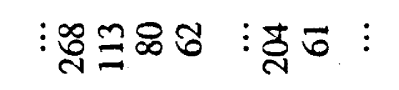 & $\vdots:$ \\
\hline ৩ 少鸟 & : : : : & $: 8 \%$ & 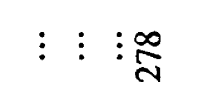 & $:::_{\infty}^{\infty} \underset{\infty}{\infty}:$ : & $\vdots \vdots$ \\
\hline o $\bar{\pi}$ & 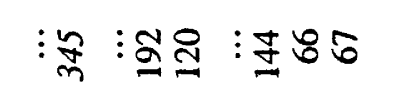 & : & :இ :@ & : & \\
\hline & $\vdots: \vdots: \curvearrowleft: \wp: \vdots$ & $: \infty \infty$ & $:::: \vdots$ & $:: \vdots: \vdots: \varrho$ & $: q$ \\
\hline & $\vdots: \vdots: \vdots: \vdots: \infty \vdots:$ & $\vdots \stackrel{\mathrm{d}}{\vdots} \vdots$ & $\approx:: \vdots$ & : : : স & \\
\hline 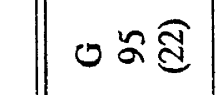 & 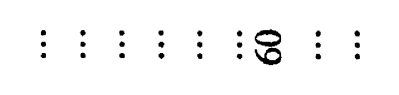 & $\vdots \stackrel{\infty}{\sim}$ & $\vdots: \vdots: \vdots$ & $\vdots:: \vdots: \vdots$ : & : \\
\hline o कి ఫ్రీ & $\vdots: \vdots: \vdots: \quad: \quad \vdots: \Xi$ & : & $\vdots: \vdots: \vdots$ & $:::: 8 \Xi \tilde{\Xi}$ & $8:$ \\
\hline & 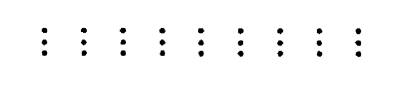 & : : & $\vdots: \vdots: \vdots$ & $\vdots: \vdots: \vdots: \infty \infty$ & $\vdots:$ \\
\hline & $\vdots: \vdots: \vdots$ : & : & $\vdots: \vdots: \vdots$ & $\vdots: \vdots: \vdots \checkmark)$ & $::$ \\
\hline$<$ & $\vdots: \vdots: \vdots: \quad: \propto \quad \vdots \quad \vdots$ & $\vdots \frac{m}{m}:$ & $\vdots: \simeq \quad \vdots$ & $\vdots: \vdots: 0: \vdots: n$ & $\vdots:$ \\
\hline & $\vdots: \vdots: \vdots: \vdots: \vdots: \vdots \bar{\pi}$ & : & $\vdots: \vdots \vdots:$ & $:::: \vdots$ 出尺 $: \vdots$ & $\vdots \vdots$ \\
\hline & 응: : : : : & : $\stackrel{0}{\Omega}:$ & $\vdots:: \infty$ & $::: 18: 8$ & $\vdots \pi$ \\
\hline 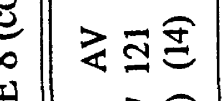 & $: \vdots:: \vdots:: \vdots: \vdots: \vdots:$ & $\vdots:$ & $\vdots: \vdots: \vdots$ & $\vdots: \vdots: \vdots: \vdots \quad \vdots$ & $\begin{array}{l}\vdots \\
\vdots\end{array}$ \\
\hline 『S & $\vdots: \vdots:: \vdots: \vdots: \vdots: q$ & స్ ิ & $\vdots: \vdots: \vdots$ & $\vdots \vdots \vdots: \vdots \infty_{\infty}^{+} \bar{m}:$ & $\vdots:$ \\
\hline 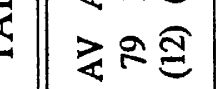 & $\vdots: \vdots: \vdots \vdots \vdots \vdots$ & 2 & $\vdots: \vdots \vdots:$ & 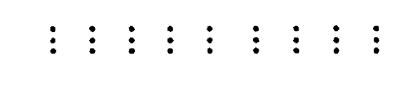 & $\vdots \vdots$ \\
\hline & 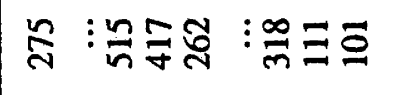 & \pm 2 & 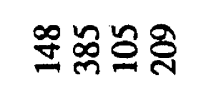 & 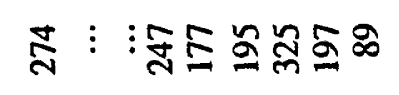 & $\S \Xi$ \\
\hline & 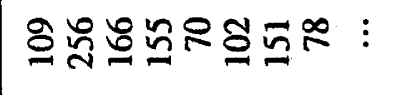 & in & $::: \vdots:$ & 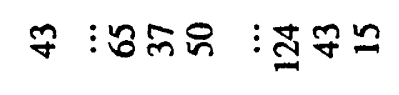 & $: \infty$ \\
\hline ఫ & 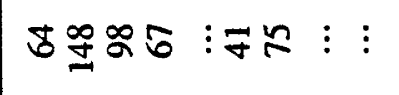 & :ฐ8 & $\vdots: \vdots \vdots \vdots$ & 뉴: $: \vdots:: \vDash: \vdots$ & $::$ \\
\hline & 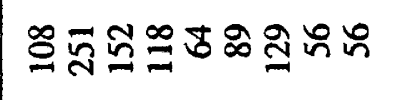 & 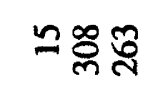 & $::: \vdots:$ & 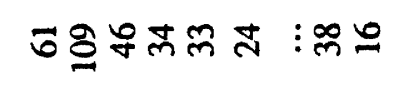 & $::$ \\
\hline ఫ్ $E$ & 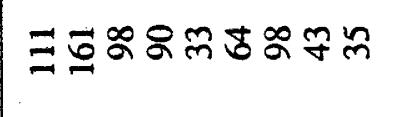 & ํํำ సิ & $\vdots: \vdots \lesssim$ & テஎ & 오 \\
\hline & 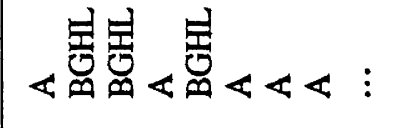 & $\sum_{3} \sum_{3}$ & 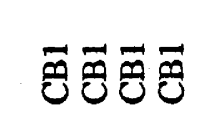 & 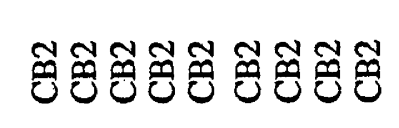 & $\sum \tilde{E}$ \\
\hline & 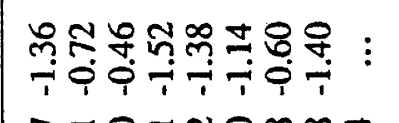 & 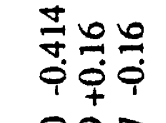 & สิธี่ & 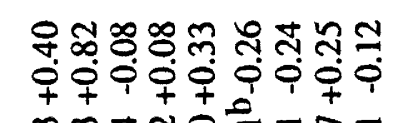 & (2) \\
\hline & 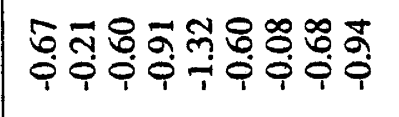 & & 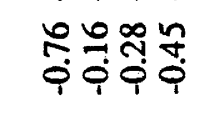 & 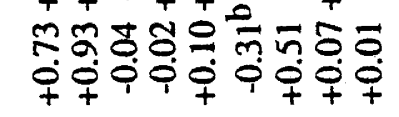 & 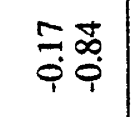 \\
\hline & 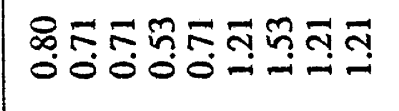 & సั. & 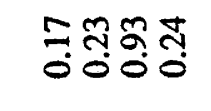 & 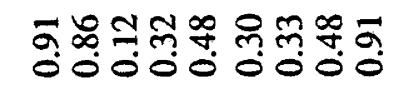 & no \\
\hline & & & & & 2 \\
\hline
\end{tabular}




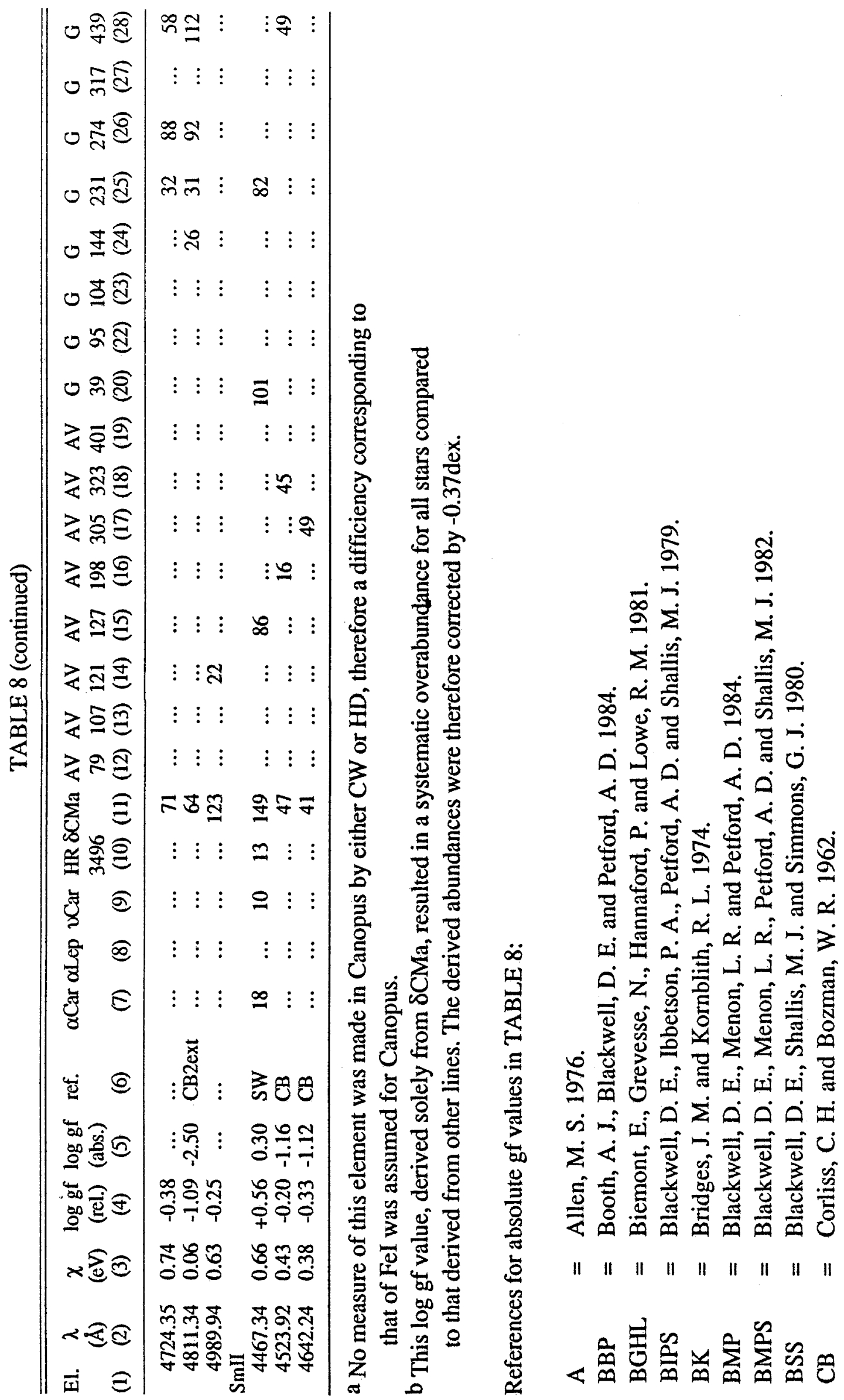




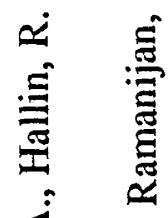

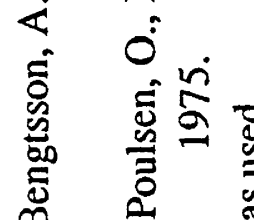

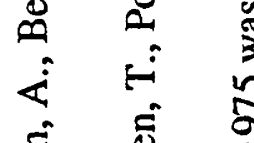

离离

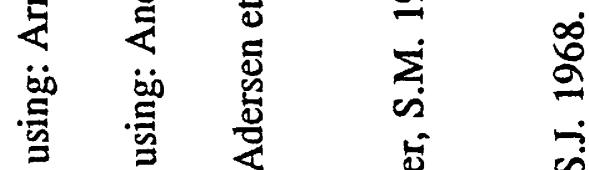

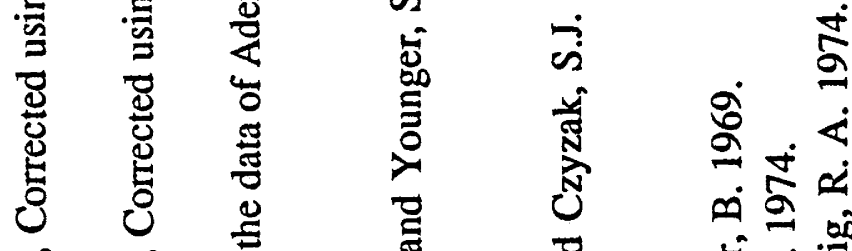

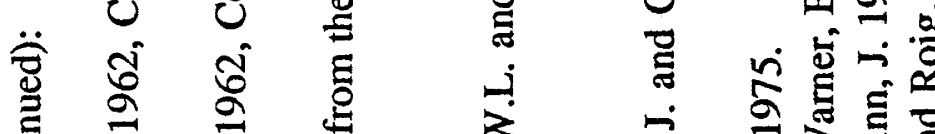

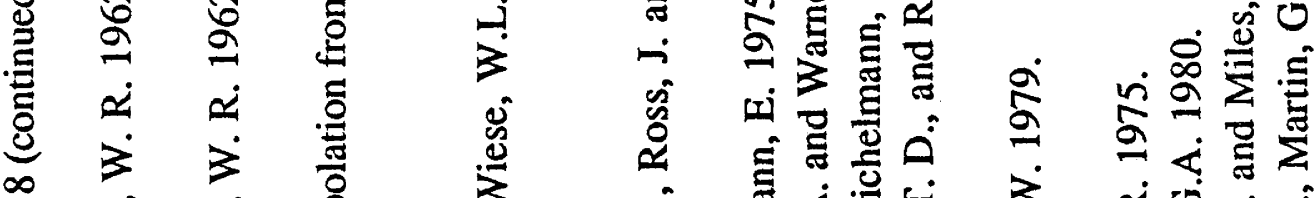

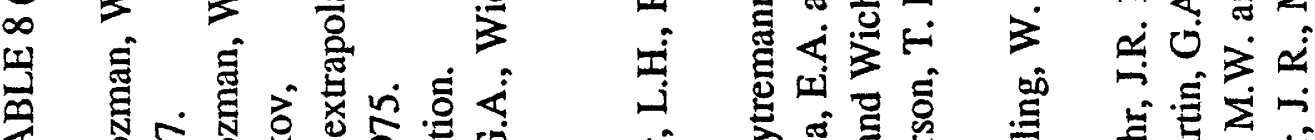

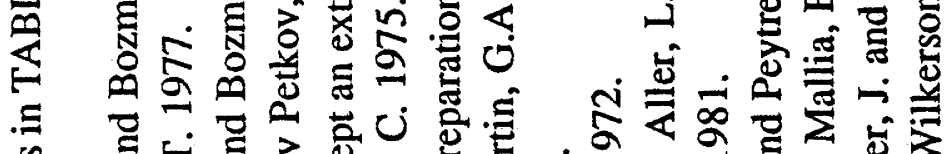

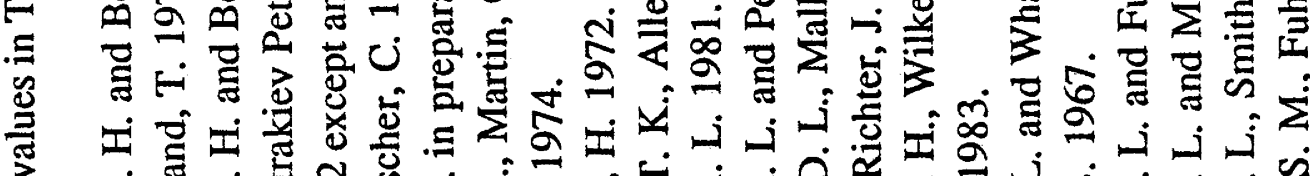
ن एँ

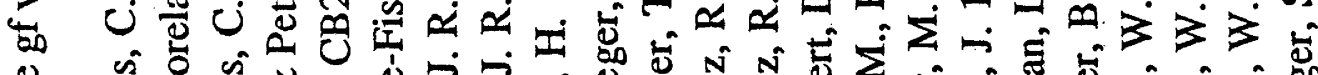

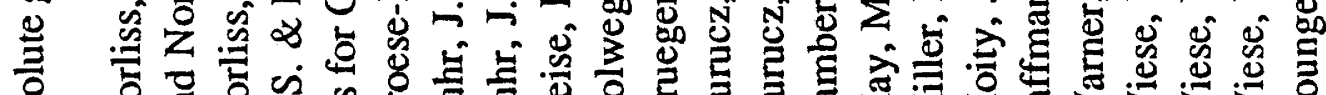

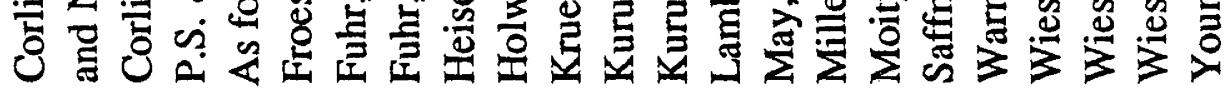

" | | || || || || || || || || || || || || || || || || || || ||

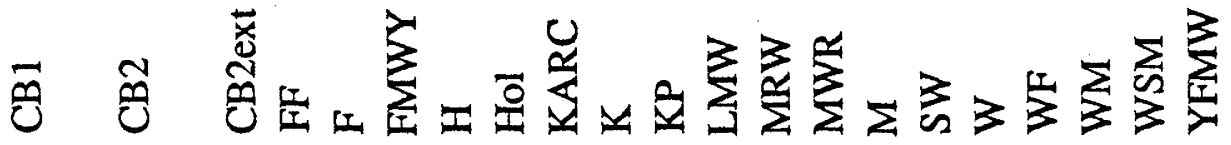


Figure 9 Graph showing the change in abundance $(\log \varepsilon)$ for any equivalent width, resulting from a $1 \mathrm{~km} \mathrm{~s}^{-1}$ change in microturbulent velocity from $4.5 \mathrm{~km}$ $\mathrm{s}^{-1}$ up to $9-10 \mathrm{~km} \mathrm{~s}^{-1}$. These curves seem insensitive to both the temperature and gravity of a star, but have only been tested over the range of physical parameters of interest here (i.e. F Ib-Ia supergiants). 


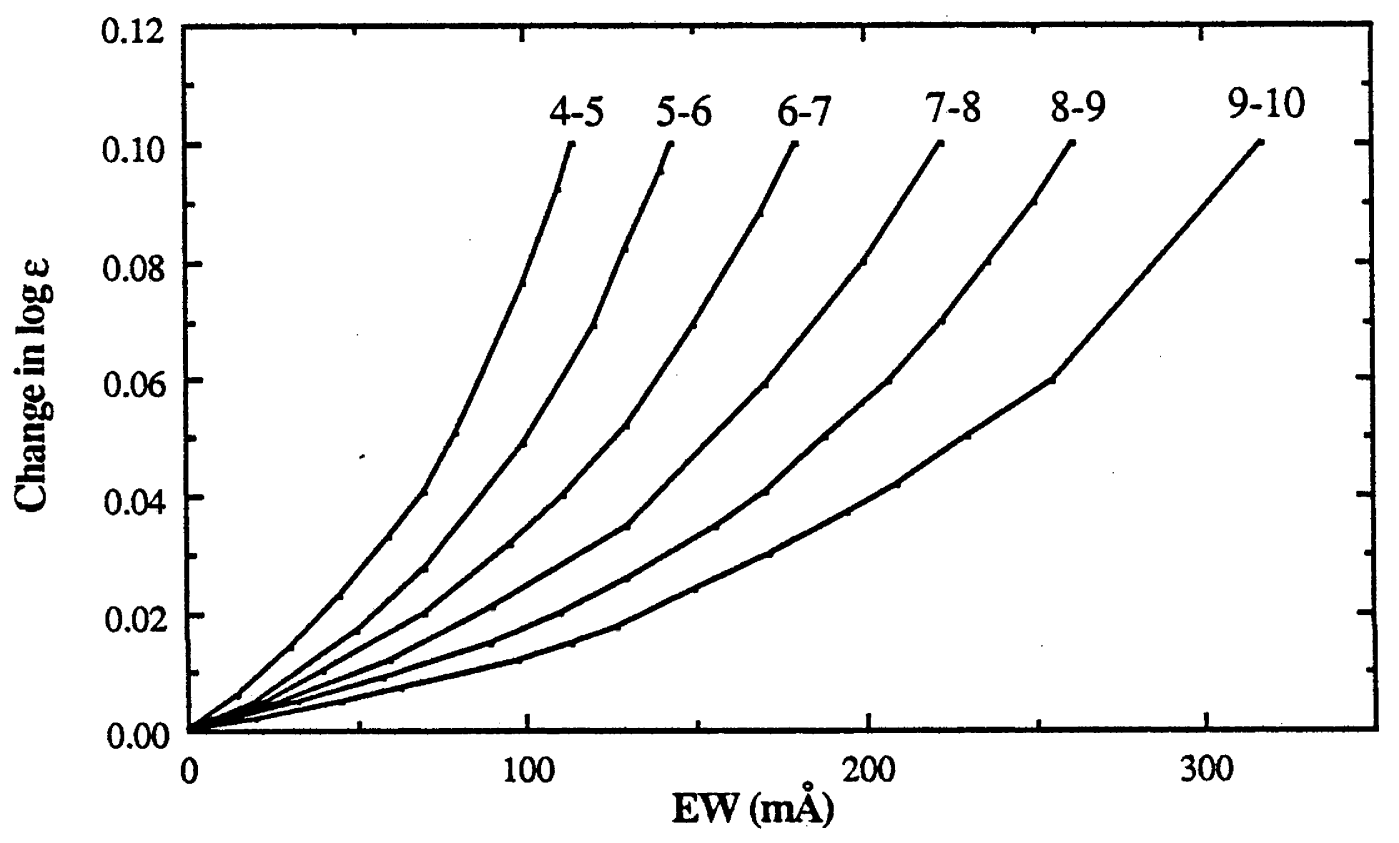


Powell 1973, Luck 1979, Pilachowski, Wallerstein, and Leep 1980, Peterson 1981, Sneden and Parthasarathy 1983, François 1986, Gratton and Sneden 1987). This can lead to large systematic errors when there are serious differences between the stellar equivalent widths and those of the Sun. The main causes for these errors are, according to Magain (1985), a poor choice of damping constants in the solar analysis and line crowding resulting in severe blending and poor continuum placement in the blue part of the solar spectrum. However, errors in $\xi_{T}$, solar equivalent widths, and non-LTE effects will also contribute to the overall error. These errors can be reduced by using relative oscillator strengths derived from stars of similar type.

Unrecognized blending of lines, even in the less crowded spectrum of an F supergiant, will still contribute a large "error" to the measurement of equivalent widths that will still remain, even if absolute oscillator strengths are used, but will largely cancel out in a differential analysis with a star of similar type. In addition, the existing absolute $g f$-values suffer badly from lack of precise experimental determinations. Both of these effects result in the sometimes large differences between the absolute and differential $g f$-values listed in Table 8.

We chose for our comparison, the F Ib-II star Canopus, which is well placed for observation in the southern hemisphere. This star has been studied in great detail recently, at a dispersion of $2 \AA \mathrm{mm}^{-1}$, both by Castley and Watson (1980) and by Hearnshaw and Desikachary (1982). We chose for our main comparison the work of Castley and Watson since they also observed several other stars that we chose as Galactic standards. The work of Hearnshaw and Desikachary was used as a backup for abundances of elements not measured by Castley and Watson and for checking the accuracy of their results. The lines we chose for analysis were derived from consideration of our own observations of Canopus and from the solar atlas prepared by Beckers, Bridges, Gilliam (1976), plus the line lists for the Sun by Moore, 
Minnaert, and Houtgast (1966), for Canopus by Hearnshaw and Desikachary (1982), and for $\alpha \operatorname{Per}$ (F5 Ib) by Dunham (1929).

We derived the abundances on the VAX 785 computer at Mount Stromlo Observatory (MSO), using the fine analysis program WIDTH6, a derivative of Kurucz's ATLAS5 code (see Kurucz 1970), and using the classical assumptions of LTE, hydrostatic equilibrium, and a plane-parallel atmosphere (the latter of which has been shown by Schmid-Burgk and Scholz [1975], Lyubimkov and Boyarchuk [1982], and Boyarchuk and Lyubimkov [1984] to hold for the types of stars we are considering). The program requires as input, the equivalent widths, the oscillator strengths, the upper and lower energy levels for each transition. The approximation we used for van der Waals damping was three times that used by Unsöld (1955), but the exact value is of little importance for us since it was shown by Boyarchuk and Lyubimkov (1982), that the contribution of the van der Waals damping to the total damping for F supergiants, is negligible for the line strengths we are interested in. Indeed, the change in abundance due to an order of magnitude error in the total damping for Canopus, only reaches 0.1 dex for $310 \mathrm{~m} \AA$ lines, as compared to the much more stringent cutoff at $120-150 \mathrm{~m} \AA$ from errors in $\xi_{T}$.

The program calculates the line opacity coefficient for 35 different depths in the chosen model atmosphere, to determine the total line absorption for an assumed elemental abundance. The calculated width is compared with the observed width, and the difference between them serves as the basis for a better guess at the abundance. The whole process is iterated until satisfactory agreement is achieved. The model atmospheres we chose were taken from the grid of constant flux, LTE, solar metallicity, atomic-line-blanketed models calculated by Kurucz (1979). One makes a series of passes through the program varying the effective temperature, gravity and microturbulent velocity, in an effort to reduce to a minimum any variation in abundance with excitation potential, equivalent width and ionization 
state. This was best done with those elements having a large number of lines covering a wide range in excitation potential and equivalent width, in both ionization states. Therefore the physical parameters of a star were determined first, using only the iron, titanium, and in some cases the chromium lines. Only then were the abundances of the other elements derived.

For $\mathrm{Mg}$, the odd elements, and the rare earths, a rough correction for hfs (hyperfine splitting) was made, where necessary, by artificially increasing the turbulent velocity. The exact amount we used was determined empirically from inspection of the Liège solar atlas (Delbouille, Neven, and Roland 1973) and from a calibration based on the work of Gratton (1982), shown in Figure 10. Here we have plotted the extra microturbulent velocities due to hfs ( $\xi_{\text {hfs }}$ ) that were derived by Gratton, against the fractional increase in the FWHM (full width at half maximum) of the lines measured in the solar atlas, compared to the FWHM of lines having the same equivalent width (as determined from the compilation of Moore, Minnaert and Houtgast 1966), but negligible hfs (e.g., Fe and Ti lines). As can be readily seen, the correlation between these two quantities is very good, and this encouraged us to use this simple method, rather than the more laborious method of actually modeling an unaffected line at a series of microturbulences, until the profile matched in some way the observed line. The extra work involved in using the latter method was certainly not warranted, given the wide differences in $\xi_{\text {hfs }}$ determined by two different authors (Gratton 1982, Cohen 1978) using this method. Further, the effects of hfs are minimized in our case due to the weakness of the majority of the lines (thus making them insensitive to $\xi_{T}$ ), and since ours is a relative abundance analysis, any errors in $\xi_{\text {hfs }}$ are largely counteracted.

For most elements, there were enough lines for us to determine the abundance without recourse to the use of resonance lines. However for two elements, $\mathrm{Sr}$ and $\mathrm{Ba}$, resonant lines were almost the only lines available for observation in our part of 
Figure 10 The increase in turbulent velocity derived by Gratton (1982) (Gratton $V t)$ in $\mathrm{km} \mathrm{s}^{-1}$, required to compensate for the fractional increase in full width at half-maximum of a spectral line, due to hyperfine splitting, over that of an unaffected line. 


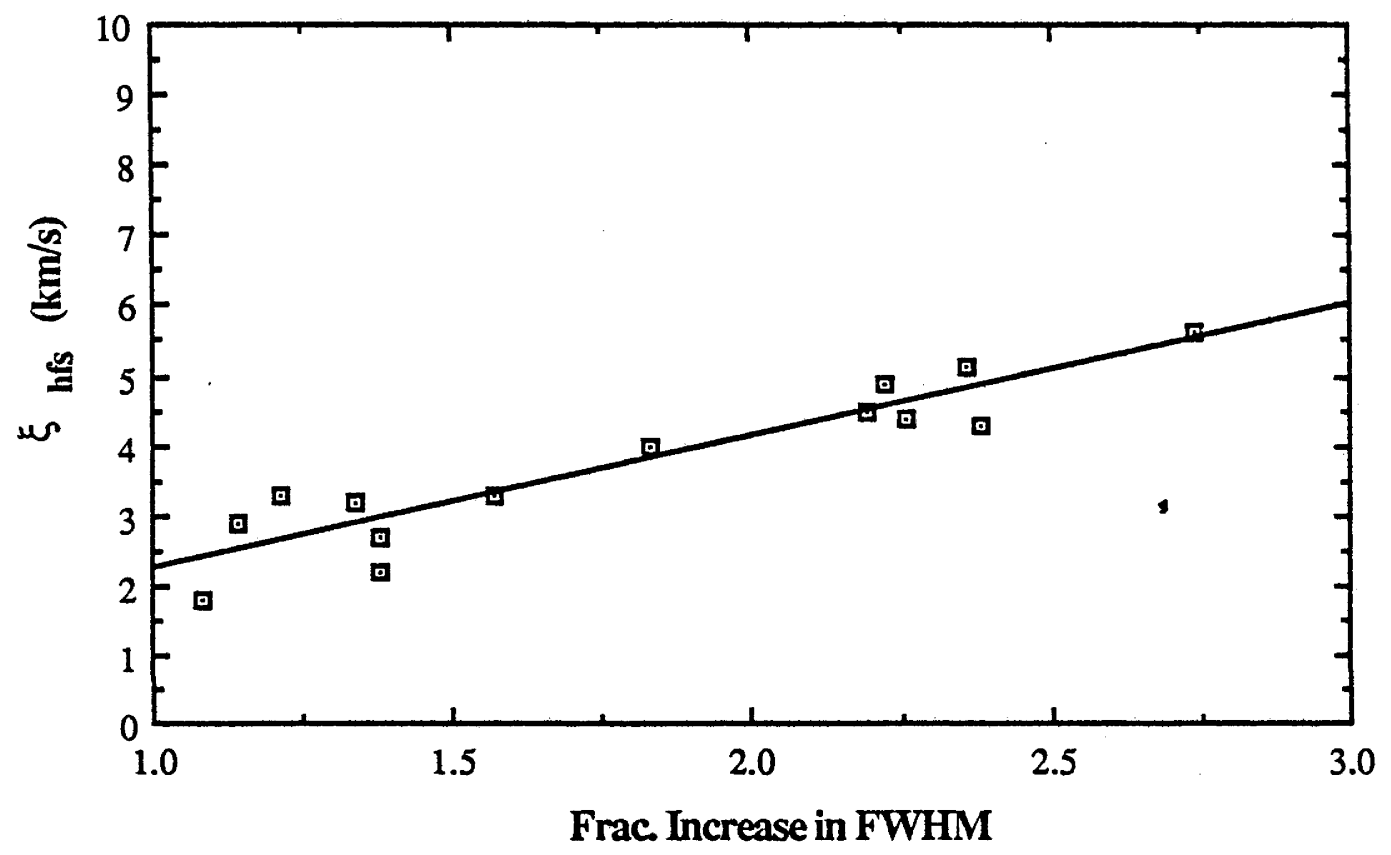


the spectrum. The case for determination of abundances from resonance lines has been discussed in some detail by Lyubimkov and Boyarchuk (1982) in relation to Canopus. They were unable to make the absolute abundances from the $\mathrm{Sr}$ and $\mathrm{Ba}$ lines agree with those derived from the nonresonant lines, even after assuming the source function for these lines was derived from pure coherent scattering. They concluded that the error was due to the effects of non-LTE, which had not been taken into account in their analysis. This means that the derived abundances depend critically upon the exact temperature structure of the star. However, since ours is a differential analysis between stars with very similar physical properties, it is hoped that this problem will be minimal.

\section{c) The Absolute Abundance Analysis of Canopus}

In order to form a strong foundation for our comparative analysis with Canopus, we first derived the absolute abundances of Canopus using the oscillator strengths (gf-values) derived from the literature (see Table 8) and the physical parameters for the star derived from the analysis of Castley and Watson (1980) by Boyarchuk and Lyubimkov (1982). The results are shown in Figures $11 a$ and $11 b$, which show the difference between our determination of the abundances in Canopus and those of Lyubimkov and Boyarchuk (1982), and between ours and those of Desikachary and Hearnshaw (1982), respectively. Our abundance measures agree very well with those of both groups of investigators and, if anything, perhaps a little better with Lyubimkov and Boyarchuk. Figure $11 c$ shows the difference between the abundance measures of Desikachary and Hearnshaw and Lyubimkov and Boyarchuk. Clearly the scatter between our measures and those of the other observers (who were using more than twice our resolution) is of the same order as the scatter between the measures of the other observers themselves. Thus we can be 
Figure 11 (a) The difference between the absolute abundances for Canopus obtained by us (RB) and those from Lyubimkov and Boyarchuk (1982) (LB) (which are derived the data of Castley and Watson [1980]), plotted against the atomic number $Z$. The open squares without error bars refer to elements represented in our spectra by less than three lines.(b) The difference between RB and Desikachary and Hearnshaw (1982) (DH). (c) The difference between DH and LB. 

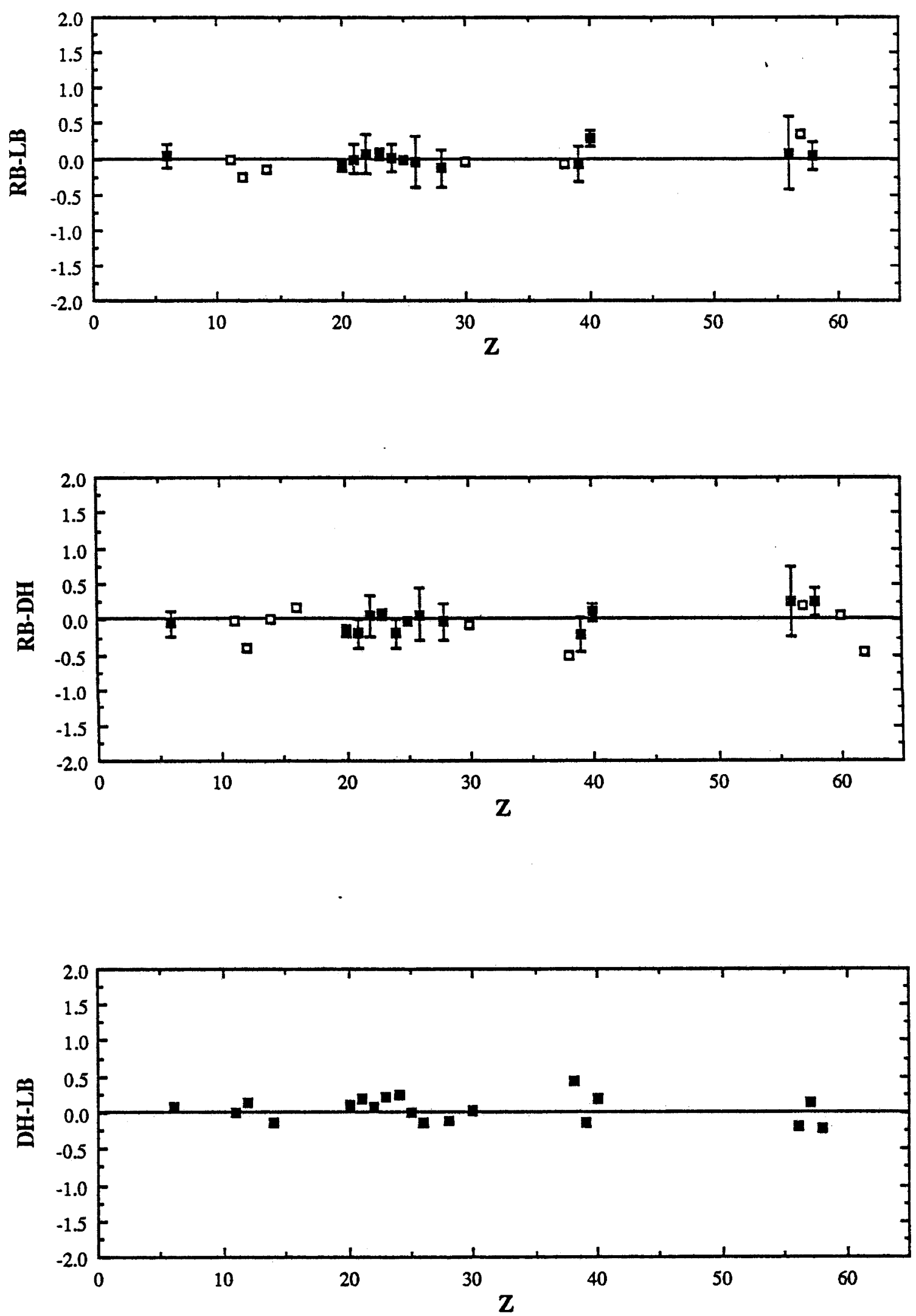
confident that our methods of analysis are sound and we may proceed to the differential analysis.

\section{d) The Differential Analysis}

Our first step was to adjust the $g f$-values so that all of the lines for a particular element give an identical abundance, in this case the abundances of the elements derived from Lyubimkov and Boyarchuk (and, where necessary, Desikachary and Hearnshaw) for Canopus. This set of $g f$-values was then applied to the other Galactic standards listed in Table 4. In all cases the scatter of abundances about the mean was sharply reduced. Part of the remaining scatter in abundances of these standards must have been derived from errors in the measurement of their own line strengths, but a part must also have been derived from errors in the measurement of line strengths for Canopus. Thus the $g f$-values were altered again so that the scatter about the mean abundance for each element was the same in both Canopus and at least one other star. It is important to note here that the spectroscopic standards we chose cover the complete range of program stars (except for AV 121), as can be seen from Figure 1, and the $g f$-values we finally chose (listed in Table 8) were just as effective in reducing the scatter about the mean abundance for each element in the F8 Ia star $\delta \mathrm{CMa}$ as in the hotter, less luminous standards. The results for all the standard stars are presented in Figures $12 a-12 e$, while the average for all the standards is given in Figure 13. As expected from the literature, the supergiants have, on average, the solar distribution of elements.

\section{e) Estimation of the Physical Parameters for the Program Stars}

The differential set of $g$-values was then used to analyze the program stars in the Magellanic Clouds. This resulted in a decrease in the scatter about the mean abundance which was dramatic in most cases. For example, Figures $14 a-14 c$ show, for the star G144, the scatter about the mean abundance of Fe I as a function 
Figure 12 (a) Measured abundances for Canopus relative to the Sun plotted against the atomic number $Z$. The open squares without error bars refer to elements represented in our spectra by less than three lines. $(b)$ Relative abundances for $\alpha$ Lep plotted against Z. (c) Relative abundances for $v$ Car plotted against Z. (d) Relative abundances for HR 3496 plotted against Z. (e) Relative abundances for $\delta$ CMa plotted against $Z$. 

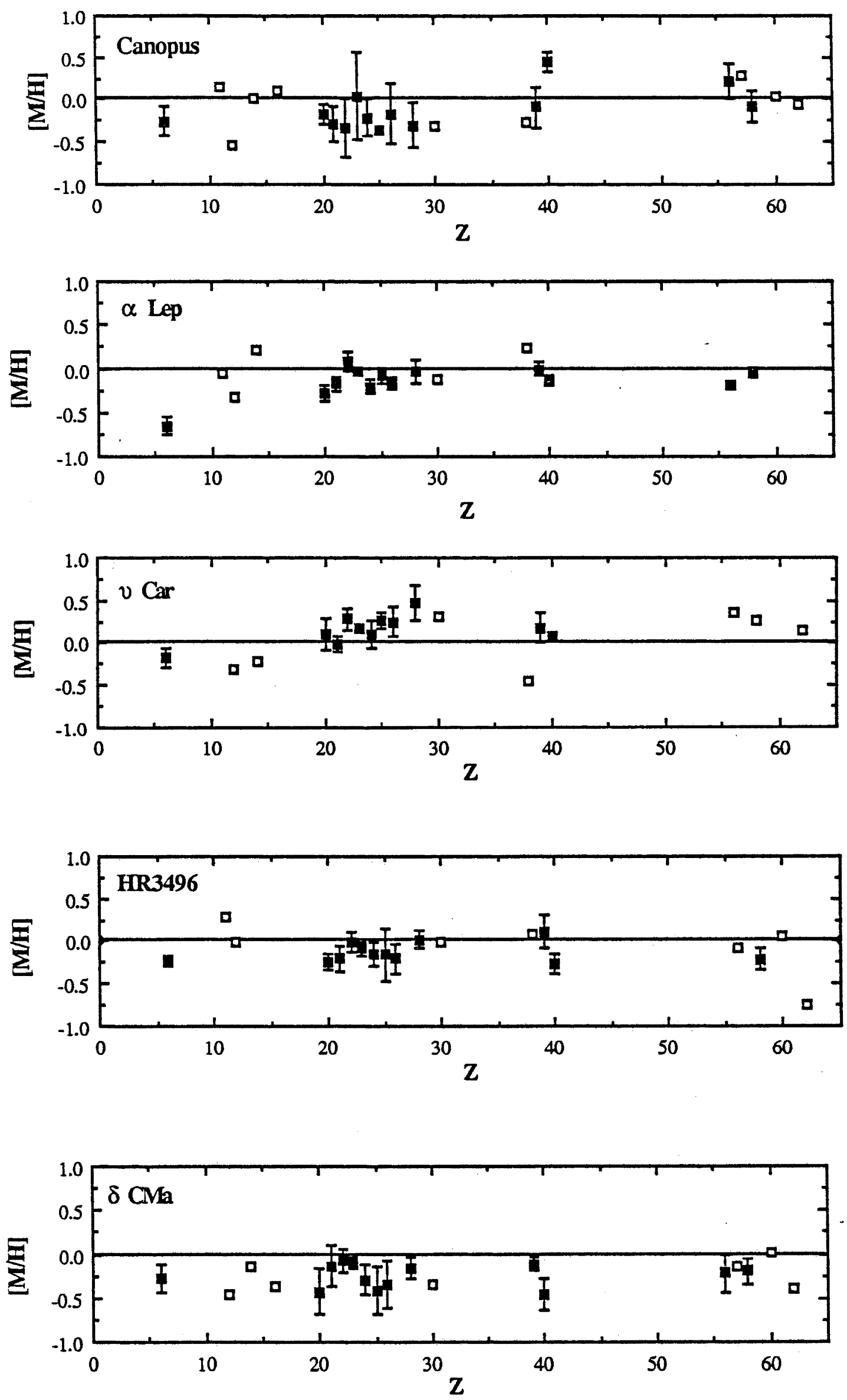
Figure 13 The average abundances of all five standards studied in this work, relative to iron. 


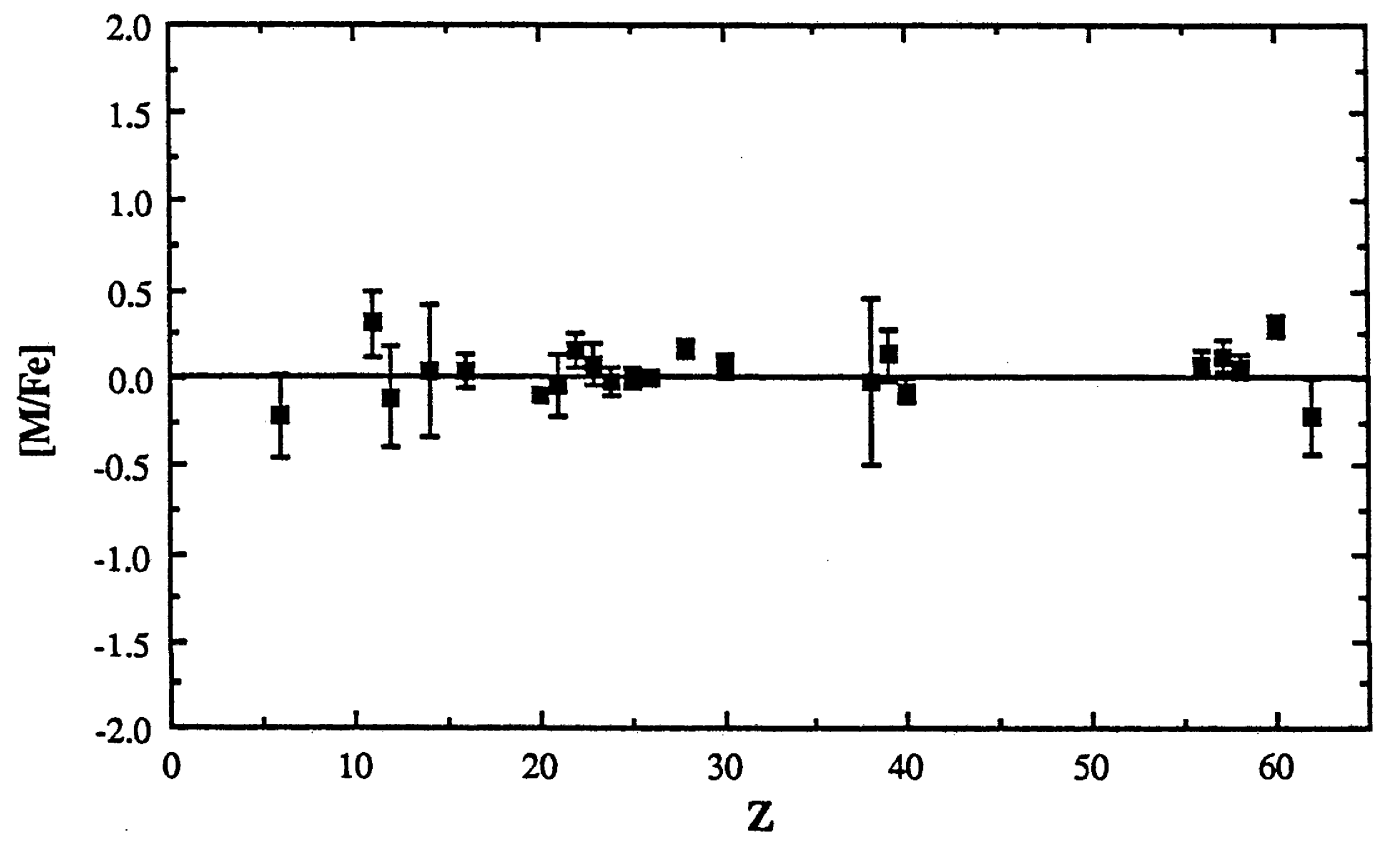


Figure 14 Typical distribution (in this case, for G144) of Fe I abundances as a function of (a) $\chi$, (b) equivalent width (EW), and (c) wavelength. 

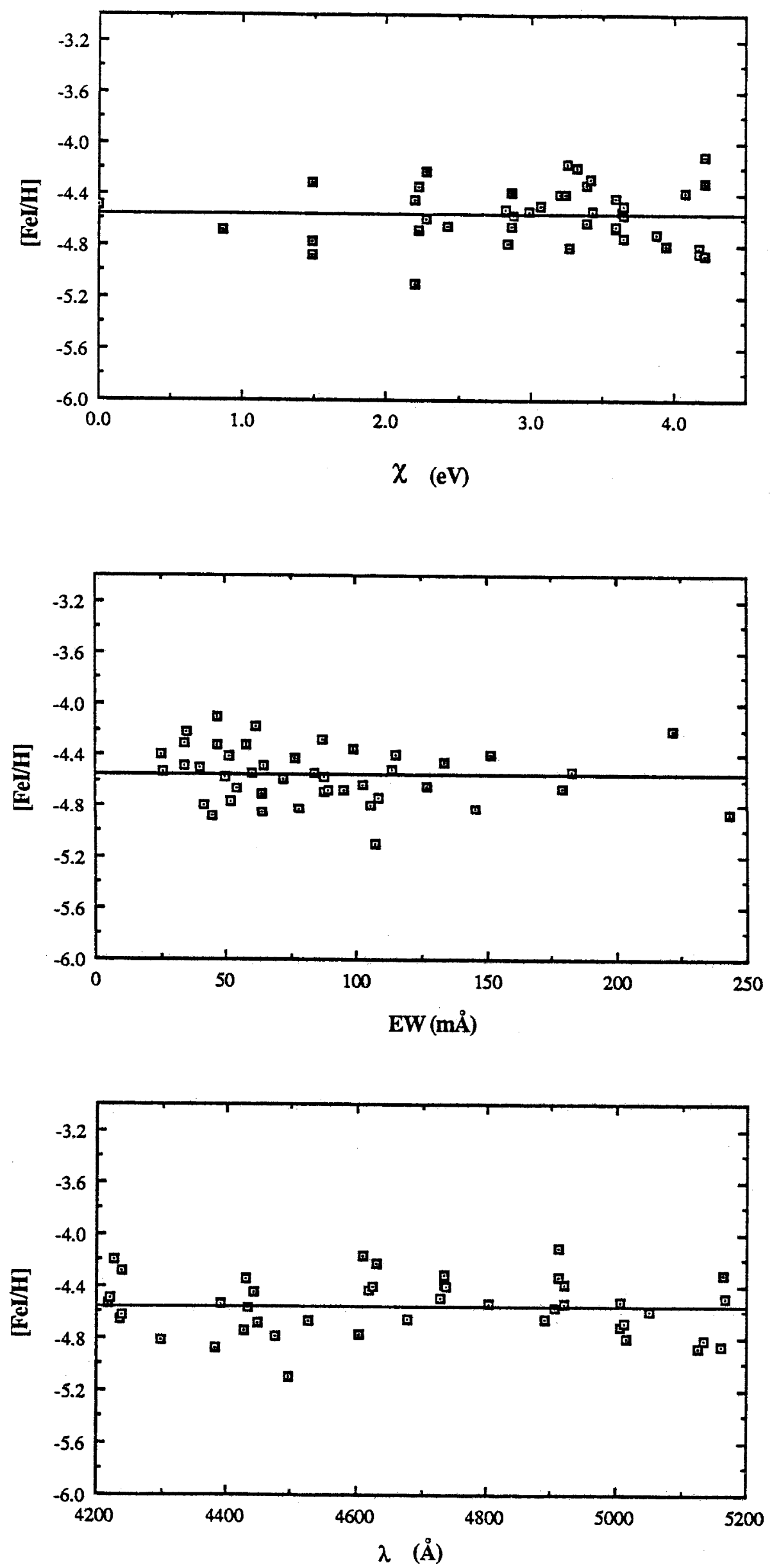
of $\chi$ (the excitation potential in $\mathrm{eV}$ ), equivalent width, and wavelength, respectively. Any errors in continuum placement, for instance, should show up as a systematic variation in abundance with wavelength.

In the first instance we derived the physical parameters of the stars, as mentioned previously, by minimizing the trends in abundance with $\chi$, equivalent width, and ionization potential for the lines of $\mathrm{Fe}$ and $\mathrm{Ti}$, and in some cases $\mathrm{Cr}$. We found that the trend in abundance with $\chi$ was relatively insensitive to effective temperature for our stars, and thus we used the photometric temperatures in every case (unless we used temperatures derived from a more detailed analysis in the literature).

The accuracy of our determination of $\xi_{T}$ depended upon the number of lines we had available, but was in most cases good to within $\pm 0.5 \mathrm{~km} \mathrm{~s}^{-1}$. However, for stars with very high values for $\xi_{T}$, the sensitivity of the trends in abundance with changes in $\xi_{T}$ decreased (see Fig. 9), resulting in less accurate determinations of this value. Fortunately, the very fact that the abundance became less sensitive to $\xi_{T}$ meant that the lower accuracy in our determinations of $\xi_{T}$ became less critical. Figure 15 shows the averaged values for $\xi_{T}$ for each spectral type, at the two ionization states, for each of the Clouds. These are compared with averaged Galactic values (drawn in as lines) derived from Osmer (1972), except for the F0 Ib stars, which were from the values we used in our analysis of Canopus and $\alpha$ Lep. It seems from this that the values of $\xi_{T}$ agree very well from one galaxy to the next and are therefore somewhat independent of metallicity.

The fine adjustment to the gravities for our stars was achieved by trying to minimize the abundance differences obtained for elements having lines derived from different ionization states. This procedure is complicated by the effects of non-LTE, known to be influential in these types of stars and discussed by, among others; Lyubimkov and Boyarchuk (1983); Boyarchuk, Lyubimkov and Sakhibullin 
Figure 15 Averaged microturbulent velocities $[(I)=$ neutral, and $(\mathrm{II})=$ ionized $]$ for the program stars (symbols as shown in the legend), and the Galaxy (plotted as lines), for luminosity classes $\mathrm{Ia}$ and $\mathrm{Ib}$. 


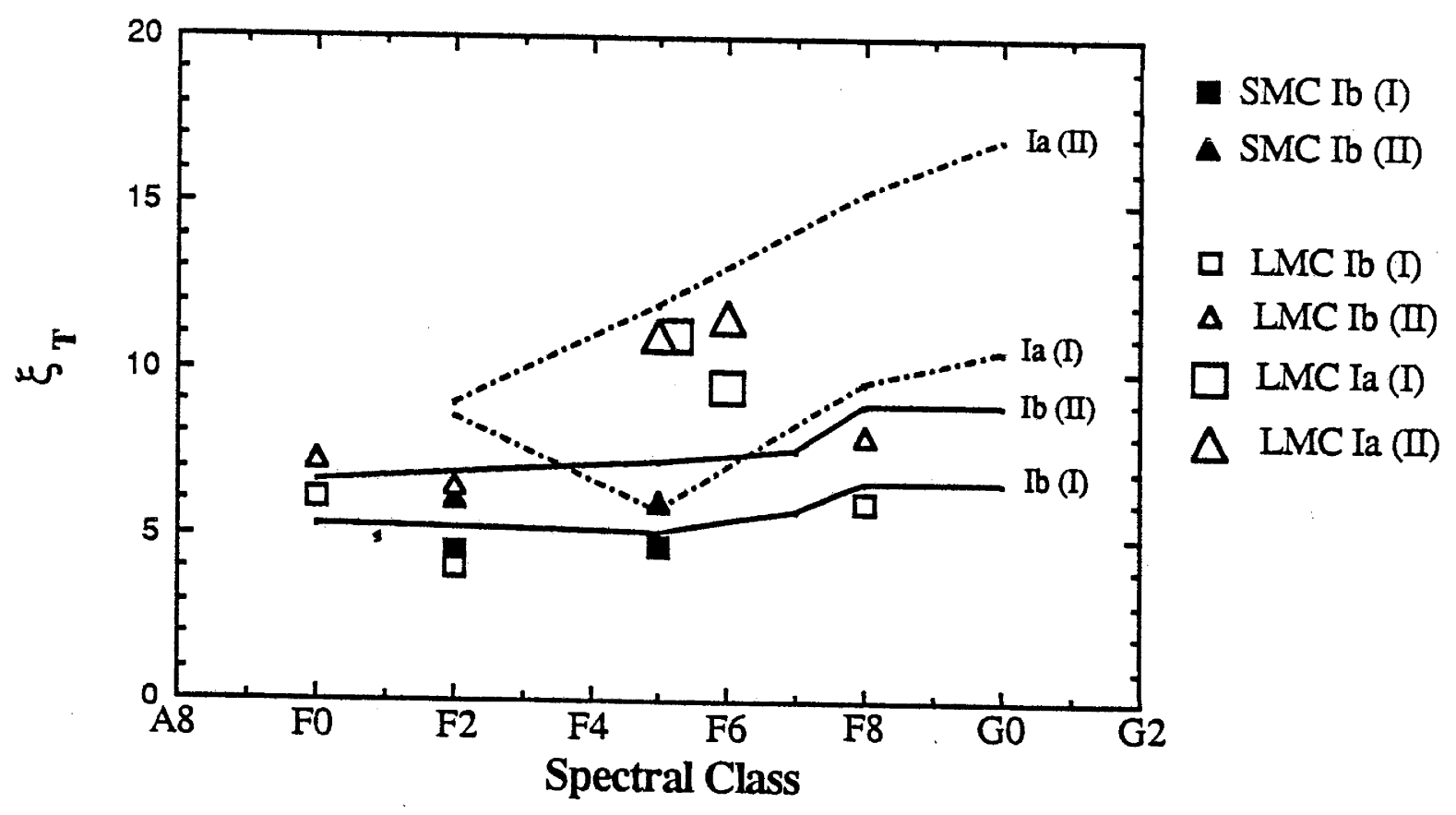


(1984): and by Spite, Spite, and François (1988). As seen in Figure 15, the microturbulent velocities derived from neutral lines in F supergiants are consistently lower than those obtained from ionized lines. The cause of this effect is the difference between the photoionization temperature and recombination temperature. Recombination occurs under the influence of the local temperature in the atmosphere, while photoionization is strongly affected by ultraviolet radiation from the deeper layers. Since most iron atoms in the atmospheres of F supergiants are in the ionized state, it is impossible to change the $N(\mathrm{Fe}$ II) concentration significantly by this effect, and the resulting increase in ionization is only evident through a decrease in the $N(\mathrm{Fe} \mathrm{I})$ concentration. Thus, if the assumption of LTE is strictly adhered to, not only is $\xi_{T}$ apparently smaller for neutral iron (as determined from the constraint that there should be no variation in abundance with equivalent width), but the abundance of iron using this value of $\xi_{T}$ is also smaller.

The error in iron abundance resulting from neglect of the overionization of $\mathrm{Fe}$ I was calculated by Lyubimkov and Boyarchuk (1983) for I Car (F0 Iab) and $\gamma$ Cyg (F8 Ib) and found to be -0.18 and -0.03 dex, respectively. Spite, Spite, and François (1988) found up to 0.1 dex deficiencies in neutral elemental abundances for the SMC star AV 369 if they assumed LTE rather than non-LTE and a massive decrease in gravity of 0.5 dex in order to retain ionization balance. Therefore, a rigid insistence on ionization balance would always result in underestimates of the gravity and the iron abundance. With these factors in mind, the gravities of the program stars were judiciously chosen in an attempt to satisfy all the available evidence. In 10 out of the total of 15 program stars, the photometric determinations of gravity were accepted. For the remaining stars, the ionization balances were used to make a more consistent determination of the gravities. The value of the photometric index, $c_{1}$, for one of these stars (AV 79) was clearly wrong due to the presence of a close companion in the aperture. Three of the remaining four stars were the only F Ia 
supergiants in the whole sample (besides AV 121 which is discussed separately). This fact alone illustrates the increased difficulties in determination of the physical parameters for the more luminous, more extreme stars. Even for these stars however, the error in gravities should not be so large as to influence the derived absolute abundances by more than $0.1 \mathrm{dex}$. The ratio of the abundance of an element relative to iron, on the other hand, would be left almost unaffected.

Table 9 gives the final adopted values for the physical parameters of the program stars and the Galactic standards. Columns (3) and (4) give the effective temperatures $\left(T_{\text {eff }}\right)$ and logarithms of the gravities $(\log g)$ respectively, while columns (10) and (11) give the microturbulent velocities (assumed constant with depth) for the neutral species $\left(\xi_{I}\right)$ and singly ionized species $\left(\xi_{I I}\right)$, respectively. Included in this compilation are the derived values for the absolute visual, magnitudes $\left(M_{V}\right)$ in column $(5)$, and absolute bolometric magnitudes $\left(M_{\mathrm{BOL}}\right)$ in column (6). The absolute visual magnitude has been determined from the formula

$$
M_{V}=m_{V}-A_{V}-\mathrm{DM} \text {, }
$$

where $m_{V}$ is the observed visual magnitude, $A_{V}$ is the visual interstellar extinction

(taken to be 3.0E[B-V] from Morgan, Harris and Johnson (1953), which corresponds to $4.05 E[b-y]$ ), and DM is the distance modulus (taken to be 18.6 for the LMC [Crampton and Greasley 1982], and 19.1 for the SMC [Crampton 1979]).

The bolometric magnitudes have been determined from their $M_{V}$ using the bolometric corrections from Johnson (1966). We determined the masses of the stars (shown in col. [7] of Table 9, in the form $\log M / M_{\odot}$, where $M$ is the stellar mass and $M_{\odot}$ is the solar mass) from the evolutionary models derived by Maeder (1981) (see Fig. 1), using case B, which corresponds to moderate mass loss. The radius of 
TABLE 9

The Physical Parameters

\begin{tabular}{|c|c|c|c|c|c|c|c|c|c|c|}
\hline $\begin{array}{l}\text { Star } \\
\text { (1) }\end{array}$ & $\begin{array}{l}\text { Spectral } \\
\text { Type } \\
\text { (2) }\end{array}$ & $\begin{array}{c}T_{\text {eff }} \\
(\mathrm{K}) \\
(3)\end{array}$ & $\underset{(4)}{\log g}$ & $\begin{array}{c}M_{y} \\
(5)\end{array}$ & $\frac{M_{\mathrm{BOL}}}{(6)}$ & $\underset{(7)}{\log }$ & $\begin{array}{c}\log \\
R / R_{\odot} \\
(8)\end{array}$ & $\begin{array}{l}\log \\
\text { gev } \\
(9)\end{array}$ & $\begin{array}{l}\xi_{I} \\
(10)\end{array}$ & $\begin{array}{l}\xi_{\text {(II }} \\
\text { (11) }\end{array}$ \\
\hline \multicolumn{11}{|c|}{ Standards } \\
\hline $\begin{array}{l}\cup \mathrm{Car} \\
\alpha \mathrm{Car} \\
\propto \mathrm{Lep} \\
\mathrm{HR} 3496 \\
\delta \mathrm{CMa}\end{array}$ & $\begin{array}{l}\text { A8Ib } \\
\text { FOIb-II } \\
\text { F0Ib } \\
\text { F2Iab } \\
\text { F8Ia }\end{array}$ & $\begin{array}{l}8000^{a} \\
7400 \\
7300 \\
7050^{c} \\
6250\end{array}$ & $\begin{array}{l}1.83^{b} \\
1.9 \\
1.75 \\
1.50^{d} \\
1.00\end{array}$ & $\begin{array}{l}-5.27 \\
-5.03 \\
-5.48 \\
-6.39 \\
-7.4\end{array}$ & $\begin{array}{l}-5.18 \\
-4.89 \\
-5.34 \\
-6.26 \\
-7.24\end{array}$ & $\begin{array}{l}0.93 \\
0.90 \\
0.95 \\
1.05 \\
1.32\end{array}$ & $\begin{array}{l}1.77 \\
1.72 \\
1.82 \\
2.00 \\
2.42\end{array}$ & $\begin{array}{l}\ldots \\
\ldots \\
\ldots \\
\ldots \\
\ldots\end{array}$ & $\begin{array}{r}5.0 \\
4.5 \\
6.0 \\
6.5 \\
14.0\end{array}$ & $\begin{array}{r}6.0 \\
5.7 \\
7.5 \\
7.7 \\
15.0\end{array}$ \\
\hline \multicolumn{11}{|c|}{$S M C$} \\
\hline $\begin{array}{l}\text { AV 79 } \\
\text { AV } 107 \\
\text { AV } 121 \\
\text { AV } 127 \\
\text { AV } 198 \\
\text { AV } 305 \\
\text { AV } 323 \\
\text { AV } 369 \\
\text { AV } 401\end{array}$ & $\begin{array}{l}\text { F2 } \\
\text { F5 } \\
\text { G0 } \\
\text { F2 } \\
\text { F5 } \\
\text { F5 } \\
\text { F2 } \\
\text { G0 } \\
\text { F2 }\end{array}$ & $\begin{array}{l}7350 \\
6770 \\
5930 \mathrm{e} \\
7000 \\
6520 \\
6725 \\
6930 \\
6450 \mathrm{f} \\
7340\end{array}$ & $\begin{array}{l}2.00 \\
1.50 \\
0.60 \\
1.50 \\
1.32 \\
1.22 \\
1.74 \\
0.60 \\
1.60\end{array}$ & $\begin{array}{l}-5.74 \\
-5.73 \\
-8.24 \\
-6.90 \\
-6.05 \\
-6.30 \\
-6.14 \\
-8.58 \\
-6.16\end{array}$ & $\begin{array}{l}-5.61 \\
-5.62 \\
-8.20 \\
-6.77 \\
-5.94 \\
-6.19 \\
-6.01 \\
-8.54 \\
-6.03\end{array}$ & $\begin{array}{l}1.03 \\
0.98 \\
1.28 \\
1.13 \\
1.04 \\
1.06 \\
1.05 \\
1.31 \\
1.06\end{array}$ & $\begin{array}{l}1.87 \\
1.95 \\
2.58 \\
2.15 \\
2.04 \\
2.07 \\
2.00 \\
2.57 \\
1.96\end{array}$ & $\begin{array}{l}1.73 \\
1.52 \\
0.56 \\
1.27 \\
1.40 \\
1.36 \\
1.49 \\
0.61 \\
1.58\end{array}$ & $\begin{array}{r}4.0 \\
4.0 \\
10.0 \\
6.0 \\
4.0 \\
6.0 \\
4.0 \\
7.0 \\
4.0\end{array}$ & $\begin{array}{r}4.0 \\
6.0 \\
10.0 \\
9.0 \\
4.0 \\
8.0 \\
6.0 \\
12.0 \\
5.5\end{array}$ \\
\hline \multicolumn{11}{|c|}{ LMC } \\
\hline $\begin{array}{l}\text { G39 } \\
\text { G95 } \\
\text { G104 } \\
\text { G131 } \\
\text { G144 } \\
\text { G231 } \\
\text { G274 } \\
\text { G317 } \\
\text { G439 } \\
\text { G440 } \\
\text { G470 }\end{array}$ & $\begin{array}{l}\text { F8 I } \\
\text { F0 I } \\
\text { F2 I } \\
\text { F0 I } \\
\text { F0 I } \\
\text { F0 I } \\
\text { F6: Ia } \\
\text { F5 Ia } \\
\text { F6 Ia } \\
\text { F0: I } \\
\text { F0 I }\end{array}$ & $\begin{array}{l}6300 \\
7230 \\
7120 \\
7390 \\
7000 \\
7030 \\
6120 \\
6800 \\
6270 \\
7000 \\
7500\end{array}$ & $\begin{array}{l}1.70 \\
2.08 \\
2.20 \\
2.18 \\
1.50 \\
1.30 \\
1.40 \\
1.50 \\
1.40 \\
1.45 \\
2.11\end{array}$ & $\begin{array}{l}-5.74 \\
-5.78 \\
-5.75 \\
-5.58 \\
-6.13 \\
-6.01 \\
-6.81 \\
-7.43 \\
-6.98 \\
-5.79 \\
-5.80\end{array}$ & $\begin{array}{l}-5.66 \\
-5.64 \\
-5.62 \\
-5.44 \\
-5.99 \\
-5.87 \\
-6.71 \\
-7.32 \\
-6.88 \\
-5.65 \\
-5.66\end{array}$ & $\begin{array}{l}1.01 \\
1.04 \\
1.03 \\
1.02 \\
1.04 \\
1.06 \\
1.13 \\
1.19 \\
1.15 \\
1.04 \\
1.03\end{array}$ & $\begin{array}{l}2.02 \\
1.90 \\
1.83 \\
1.99 \\
1.96 \\
2.25 \\
2.28 \\
2.26 \\
1.92 \\
1.89 \\
1.86\end{array}$ & $\begin{array}{l}1.41 \\
1.67 \\
1.80 \\
1.50 \\
1.58 \\
1.07 \\
1.07 \\
1.07 \\
1.64 \\
1.70 \\
1.75\end{array}$ & $\begin{array}{r}6.0 \\
5.0 \\
4.0 \\
\ldots .0 \\
7.5 \\
9.0 \\
11.0 \\
10.0\end{array}$ & $\begin{array}{r}8.0 \\
6.5 \\
6.5 \\
7.5 \\
7.5 \\
7.7 \\
11.2 \\
11.0 \\
12.0\end{array}$ \\
\hline
\end{tabular}

Note.- Reference for $\alpha \mathrm{Car}$ and $\alpha$ Lep in cols. (3)-(8) is Boyarchuk and Lyubimkov 1984. Reference for $\delta \mathrm{CMa}$ in cols. (2)-(3) is Luck 1979. Reference for $v \mathrm{Car}$, HR 3496, and $\delta \mathrm{CMa}$ in cols. (5)-(8) is Parsons and Bouw 1971.

a Derived from the spectral-type using the calibration of Johnson (1966).

$\mathrm{b}$ Derived from photometry after subtraction of companion.

c Derived from photometry.

d Derived from ionization balance.

e Taken from Thévenin and Foy 1986.

f Taken from Spite, Spite and Francois 1988. 
the stars could then be calculated using the formula

$$
\log \left(R / R_{\odot}\right)=\left(4.79-M_{\mathrm{BOL}}\right) / 5-2 \log \left(T_{\text {eff }} / T_{\odot}\right),
$$

where $R$ is the stellar radius, $R_{\odot}$ is the solar radius, and $T_{\odot}$ is the solar effective temperature. These results are given in column (8) of Table 9, and from these an entirely independent estimate for the gravity (the evolutionary gravity, $g_{\mathrm{ev}}$ ) could be obtained from the formula

$$
\log g_{\mathrm{ev}}=4.44+\log \left(M / M_{\odot}\right)-2 \log \left(R / R_{\odot}\right) .
$$

The results are presented in column (9) of Table 9. The difference between our accepted gravities and these evolutionary gravities is:

$$
\log g-\log g_{\mathrm{ev}}=0.17+0.24,
$$

which suggests to us that Maeder's case B somewhat overestimates the mass loss of these stars. Further evidence for this comes from details now emerging from models of the precursor star for supernova SN 1987A (Woosley 1988, Höflich 1988), which indicate a mass-loss for this star of around half that predicted by Maeder's case B.

\section{f) The Accuracy in the Determination of the Abundances}

There are two types of error we need to consider in this analysis: (a) errors that act on a single line and $(b)$ errors that act on all the lines together (typically errors in the model parameters: effective temperature, gravity, microturbulent velocity, and overall metallicity). Let us first consider the errors that act on each line individually.

We estimated the random observational errors in our equivalent widths, from the 220 lines in the program stars that had more than one measurement. The errors depended somewhat on the strength of the line, so we binned them as shown in Table 10. 
TABLE 10

Measurement Errors in

Log Equivalent Width

\begin{tabular}{|c|c|c|}
\hline 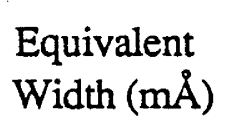 & $\begin{array}{l}\text { Error in } \\
\log E W(\text { dex })\end{array}$ & $\begin{array}{l}\text { Number } \\
\text { of lines }\end{array}$ \\
\hline $0-100$ & 0.08 & 47 \\
\hline $100-200$ & 0.06 & 67 \\
\hline$>200$ & 0.04 & 106 \\
\hline
\end{tabular}


On top of these we should add the errors due to the scatter in deduced abundances for the Galactic standards that we used to derive the stellar $g f$-values. For iron, we measured this scatter to be $\pm 0.08 \mathrm{dex}$, which combines with our errors in equivalent widths to give a total line-to-line error of $\pm 0.12 \mathrm{dex}$. This compares favorably with our observed, average, line-to-line scatter in deduced abundances for the program stars of $\pm 0.16 \mathrm{dex}$ (for elements with three or more lines). The extra error in the latter case comes from the combined effects of all other errors not specifically considered previously, including, for instance, the differences in unrecognized blending of lines in the program stars as compared to the Galactic standards.

Let us now consider the errors resulting from uncertainties in the model parameters. These, it must be remembered, are mostly canceled out when we calculate element to iron ratios. They are therefore only important for us when we are deriving the absolute iron abundances of the stars.

As mentioned previously, our errors in abundance resulting from errors in $\xi_{T}$ are quite variable depending on the actual value of $\xi_{T}$ (see Fig. 9) and the number and quality of the lines available. Usually $\xi_{T}$ was estimated to within $\pm 0.5 \mathrm{~km} \mathrm{~s}^{-1}$, but for the stars with higher values of $\xi_{T}$, errors of up to $\pm 2.0 \mathrm{~km} \mathrm{~s}^{-1}$ can occur. Nevertheless, it is estimated that the error in abundance due to this is never more than 0.1 dex in absolute terms, except where we have had to rely on the analysis of only a few very strong lines ( $\mathrm{Sr}$ and $\mathrm{Ba}$ ). In these cases the abundances could be in error by up to 0.5 dex.

The errors in photometry and derived reddening combine to give an estimated error of $\pm 200 \mathrm{~K}$ in the effective temperature, which results in an error of 0.16 dex for neutral and 0.06 dex for ionized species. Both of these errors are of the same sign, therefore their combined effect is an error of \pm 0.11 dex in the estimate of absolute abundance. Similarly, errors in photometry result in an estimated error of 
\pm 0.3 dex in $\log g$, which in turn results in an error in abundance of 0.03 dex for neutral and 0.09 for ionized species. These are of opposite sign and therefore combine to give an estimated error in abundance of only \pm 0.03 dex.

The error in abundance resulting from our use of solar metallicity model atmospheres is negligible $(<0.01 \mathrm{dex})$. The only other error that concerns us is the error in the relative abundance of our standard, Canopus, compared to the Sun. In particular, we are interested in the error in the relative abundance of iron, which, from a comparison of the results of Castley and Watson (1980) with those of Hearnshaw and Desikachary (1982), we estimated to be of the order of 0.1 dex.

The total error, $\sigma_{\text {TOT, }}$ is estimated from the formula

$$
\sigma_{\mathrm{TOT}^{2}}=\sigma_{1}^{2} / N+\sigma_{2}^{2}
$$

where $\sigma_{1}$ is the single line error, $N$ is the number of lines used to determine the abundance of a particular element, and $\sigma_{2}$ is the error due to uncertainties in the physical parameters. Clearly, for the determination of absolute abundances of iron, the error due to line-to-line variations becomes negligible. Thus we estimate the error in our absolute iron abundances to be of the order of \pm 0.2 dex.

For the ratio of elements to iron, measured differentially with respect to Canopus, the total systematic errors reduce down to \pm 0.03 dex. The most important contribution to the total error is therefore the line-to-line errors, at least for elements having less than 30 lines. The total errors, then, should be less than \pm 0.1 dex for most elements, although this will be an underestimate for elements with only one or two very weak, or very strong, lines. 


\section{ELEMENTAL ABUNDANCES}

a) The Overall Results

Tables $11 \mathrm{~A}$ and 11B present a summary of abundance results for the SMC and the LMC, respectively. Column (2) in both tables gives our adopted values for the solar abundances $(N(\mathrm{M} / \mathrm{H}))$, where $\mathrm{M}$ indicates any heavy element and $N$ indicates that the ratio is defined in terms of numbers of atoms. These values have been taken from Cameron (1982b) except where indicated. Column (3) represents our accepted values for Canopus (where we adopt the usual convention that $[\mathrm{M} / \mathrm{H}]$ $=\left(N(\mathrm{M} / \mathrm{H})_{\text {STAR }}-N(\mathrm{M} / \mathrm{H})_{\odot}\right)$. These values have been taken from the analysis of Castley and Watson (1980) made by Lyubimkov and Boyarchuk (1982), except where indicated. The other columns give the $[\mathrm{M} / \mathrm{H}]$ for each of the program stars, followed in parentheses, by the number of lines used to derive that value. In order to derive the $[\mathrm{M} / \mathrm{H}]$ for the Cloud stars from the differential abundances with Canopus, we have had to add the $[\mathrm{M} / \mathrm{H}]$ for Canopus from column (3) to obtain the differential abundances compared with the sun. The average abundances for each Cloud have then been summarized in Table 12 , which also gives the standard deviations $(\sigma)$ from the mean for each element that has more than two representative stars with measures for that element.

These average abundances are presented graphically in Figures $16 a$ and $16 b$. In each case a solid line is drawn through solar metallicity and a dashed line through the metallicity of the Cloud. All errors in these figures were taken directly from Table 12. The filled squares without error bars indicate that there were less than three stars with measures for this quantity, so the standard deviation from the mean 
TABLE 11A

Relative Abundances in the SMC

\begin{tabular}{|c|c|c|c|c|c|c|c|c|c|c|}
\hline $\begin{array}{l}\text { El. } \\
(1)\end{array}$ & $\begin{array}{c}\text { Sun } \\
(2)\end{array}$ & $\begin{array}{c}\alpha \mathrm{Car} \\
(3)\end{array}$ & $\begin{array}{l}\text { AV79 } \\
(4)\end{array}$ & $\begin{array}{l}\text { AV107 } \\
(5)\end{array}$ & $\begin{array}{c}\text { AV12 } \\
(6)\end{array}$ & $\begin{array}{l}\text { AV127 } \\
(7)\end{array}$ & $\begin{array}{l}\text { AV198 } \\
\text { (8) }\end{array}$ & $\begin{array}{c}\text { AV305 } \\
\text { (9) }\end{array}$ & $\begin{array}{l}\text { AV323 } \\
\text { (10) }\end{array}$ & $\begin{array}{c}\text { AV401 } \\
(11)\end{array}$ \\
\hline & & & & & & & & & & \\
\hline & & & & & & & & & & \\
\hline & & & & & & & & & $(1)$ & $-0.46(1$ \\
\hline $\mathrm{CaI}$ & -5.63 & $-0.25^{b}$ & $\cdots$ & 2) & $-0.87(5)$ & -0.6 & -0.69 & $-0.57(3)$ & & $-0.32(3)$ \\
\hline Call & $\ldots$ & $-0.25^{c}$ & . & $\ldots$ & $\ldots$ & $-0.3^{7}$ & $\ldots$ & & & \\
\hline $\mathrm{cm}$ & -8.93 & $-0.22^{b}$ & & $0.56(1)$ & & $-c$ & & -0.52 & (3) & $-0.86(3)$ \\
\hline 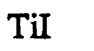 & $-6.92^{d}$ & +0.01 & & 1) & & & -0.70 & & & \\
\hline & & & -0.72 & & -0.4 & -0.53 & & -0.7 & & -0 \\
\hline VI & -8.02 & $-0.10^{e}$ & 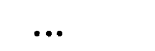 & -0.1 & 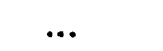 & $\ldots$ & -0.1 & & (1) & \\
\hline $\mathrm{CrI}$ & -6.32 & -0.2 & & & -0.4 & -0.17 & & -0. & & \\
\hline & & & -0.76 & & & & & & & \\
\hline InI & -6.4 & & & & -0.5 & -0.3 & & & & \\
\hline & $-4.4^{\prime}$ & & -0.87 & & -0.6 & -0. & & & & \\
\hline 10 & $\ldots$ & -0 . & -1.02 & & & & & & & -0.5 \\
\hline $\mathrm{NiI}$ & -5.75 & $0.00^{\mathrm{e}}$ & ... & -0.68 & -0.21 & -0.4 & -0.1 & -0.59 (1) & & . \\
\hline NiII & $\ldots$ & $+0.09 \mathrm{~b}$ & & 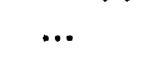 & & -0.2 & 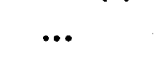 & & & \\
\hline $\mathrm{CuI}$ & -7.69 & $+0.12^{\mathrm{f}}$ & & $\ddot{~}$ & 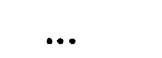 & -0.0 & 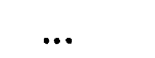 & $-0.48(1)$ & & \\
\hline SrII & -9.07 & je & . & $\cdot$ & 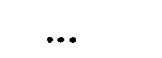 & -1.6 & & & & \\
\hline & -9.74 & & ... & -0.27 & 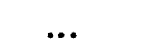 & & -0.8 & & & \\
\hline & & & & & & -0 & & & & -0.4 \\
\hline $\mathbf{n}$ & -9.35 & $-0.15 \mathrm{~g}$ & $\ldots$ & $-0.48(1)$ & & -0.5 & -0.96 & $-0.76(1)$ & $-0.69(2)$ & 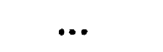 \\
\hline$\pi$ & -9.74 & $0.00^{\mathrm{b}}$ & -1.96 & $-0.97(1)$ & -1.25 & -1.2 & $-0.96(1)$ & -0.8 & -0.30 & -0.62( \\
\hline$\pi$ & -10.86 & $-0.05^{b}$ & $\ldots$ & $\ldots$ & & & & & & \\
\hline eII & -10.35 & $-0.06^{b}$ & 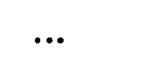 & $-0.59(3)$ & $-0.10(2)$ & -0.41 & $-0.87(2)$ & $-0.50(2)$ & $-0.35(3)$ & -0.53 \\
\hline NdII & -10.53 & $+0.17^{\mathrm{f}}$ & $\cdot$. & $\ldots$ & 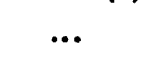 & $+0.02(2)$ & $\ldots$ & 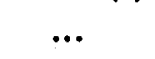 & & $\ldots$ \\
\hline $\mathrm{mII}$ & -11.04 & $-0.30^{f}$ & & 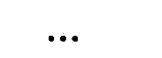 & & $+0.03(1)$ & $-0.59(1)$ & +0.11 & $+0.30(1)$ & 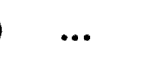 \\
\hline
\end{tabular}

Note. - The sun is measured relative to $\mathrm{H}$, Canopus relative to the Sun, and the Cloud stars relative to Canopus.

a Includes, in addition to those lines measured by SCR, those lines from Thévenin and Foy (1986) for which we have relative log gf's .

b Differs by more than 0.1 dex from Desikachary and Heamshaw 1982 (but less than $0.2 \mathrm{dex}$ ).

c Species not measured for Canopus by Lyubimkov and Boyarchuk 1982; therfore relative abundance from other species used.

d From Blackwell et al. 1982 .

e Differs by more than 0.2 dex from Desikachary and Hearnshaw 1982 (but less than 0.3 dex).

f From Desikachary and Hearnshaw 1982.

g Differs by more than $0.3 \mathrm{dex}$ from Desikachary and Heamshaw 1982 (but less than $0.4 \mathrm{dex}$ ).

$\mathrm{h}$ Not used in average Cloud abundances. 


\section{TABLE 11B}

Relative Abundances in the LMC

\begin{tabular}{|c|c|c|c|c|c|c|c|c|c|c|}
\hline $\begin{array}{l}\text { Elem } \\
(1)\end{array}$ & $\begin{array}{l}\text { Sun } \\
\text { (2) }\end{array}$ & $\begin{array}{c}\alpha \mathrm{Car} \\
(3)\end{array}$ & $\begin{array}{c}\text { G39 } \\
(4)\end{array}$ & $\begin{array}{c}\text { G95 } \\
(5)\end{array}$ & $\begin{array}{c}\text { G104 } \\
(6)\end{array}$ & $\begin{array}{c}\mathrm{G} 144 \\
(7)\end{array}$ & $\begin{array}{c}\text { G231 } \\
(8)\end{array}$ & $\begin{array}{c}\mathrm{G} 274 \\
(9)\end{array}$ & $\begin{array}{r}\mathrm{G} 317 \\
(10)\end{array}$ & $\begin{array}{c}\text { G439 } \\
(11)\end{array}$ \\
\hline & -3.38 & -0.29 & & & & $-c$ & 3) & & -0.4 & $-0.80(3)$ \\
\hline & -5.65 & +0.23 & & & & & $+0.80(1)$ & & & \\
\hline IgI & -4.40 & -0.03 & . & & & $0.00(1)$ & $-0.07(2)$ & $-0.07(1)$ & $-0.16(2)$ & $-0.33(2)$ \\
\hline iilI & -4.42 & $-0.05^{a}$ & $\cdots$ & $\cdots$ & $\cdots$ & $+0.10(1)$ & ... & $\ldots$ & $\ldots$ & $+0.66(2)$ \\
\hline SI & -4.73 & $0.00^{\mathrm{a}}$ & $\ldots$ & $\ldots$ & $\ldots$ & $\ldots$ & $\ldots$ & $\ldots$ & $\ldots$ & $-0.44(1)$ \\
\hline aI & -5.63 & $-0.25^{b}$ & $-0.38(2)$ & $-0.45(1)$ & $-0.55(3)$ & $-0.43(3)$ & $-0.29(5)$ & $-0.81(2)$ & $-0.36(4)$ & $-0.53(3)$ \\
\hline cII & -8.93 & $-0.22^{b}$ & $\ldots$ & $-0.58(3)$ & $-0.15(2)$ & $-0.74(6)$ & $-0.55(5)$ & $-0.27(1)$ & $-0.28(6)$ & $-0.43(4)$ \\
\hline $\begin{array}{l}\text { TiI } \\
\text { TiII }\end{array}$ & $\begin{array}{c}-6.92^{C} \\
\ldots\end{array}$ & $\begin{array}{r}+0.01 \\
-0.10\end{array}$ & $\begin{array}{l}-0.39(1) \\
-0.18(2)\end{array}$ & -0.33 & -0.17 & $\begin{array}{l}-0.07(2) \\
-0.28(13)\end{array}$ & $\begin{array}{l}-0.28(2) \\
-0.22(14)\end{array}$ & $\begin{array}{l}-0.16(1) \\
-0.17(15)\end{array}$ & $\begin{array}{l}-0.34(1) \\
-0.23(26)\end{array}$ & $\begin{array}{l}-0.13(2) \\
-0.24(19)\end{array}$ \\
\hline $\begin{array}{l}\text { VI } \\
\text { VII }\end{array}$ & $\begin{array}{c}-8.02 \\
\ldots\end{array}$ & $\begin{array}{l}-0.10^{d} \\
-0.15\end{array}$ & $\ldots$ & $\ldots$ & $\ldots$ & $\begin{array}{c}+0.27(2) \\
\ldots\end{array}$ & $\begin{array}{r}+0.43(2) \\
0.00(2)\end{array}$ & $-0.10(2)$ & $+0.09(4)$ & $\begin{array}{r}-0.23(1) \\
+0.01(2)\end{array}$ \\
\hline CrI & -6.32 & $\begin{array}{l}-0.21^{b} \\
-0.01\end{array}$ & $\begin{array}{l}-0.40(3) \\
-0.16(1)\end{array}$ & $\begin{array}{l}-0.27(2) \\
-0.27(4)\end{array}$ & $\begin{array}{l}-0.37(1) \\
-0.10(2)\end{array}$ & $\begin{array}{l}-0.09(3) \\
-0.32(5)\end{array}$ & & & & \\
\hline InI & -6.46 & -0.14 & $\ldots$ & ... & $\ldots$ & $-0.49(1)$ & $0.00(5)$ & $-0.31(1)$ & 2) & $(2)$ \\
\hline InII & & $-0.14^{e}$ & & & & & & & +0.0 & (1) \\
\hline $\begin{array}{l}\mathrm{FeI} \\
\mathrm{FeII}\end{array}$ & -4.47 & $\begin{array}{l}-0.12 \\
-0.10\end{array}$ & $\begin{array}{l}-0.58(4) \\
-0.40(2)\end{array}$ & & & $\begin{array}{l}-0.23(33) \\
-0.23(6)\end{array}$ & & & $\begin{array}{r}-0.38(46) \\
+0.06(10)\end{array}$ & $\begin{array}{l}-0.49(49) \\
-0.20\end{array}$ \\
\hline NiI & -5.75 & $0.00^{d}$ & $-0.22(1)$ & ... & ... & -0.0 & $-0.17(4)$ & $-0.25(3)$ & $-0.24(5)$ & $3(10)$ \\
\hline $\mathrm{ZnI}$ & -7.32 & -0.01 & $\ldots$ & 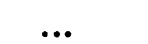 & & -0.3 & -0.3 & ) & & $-0.51(1)$ \\
\hline SrII & -9.07 & $-0.15^{d}$ & $\ldots$ & ... & $\ldots$ & $-0.68(1)$ & $-0.65(1)$ & $-0.53(1)$ & +0.54 & $-0.97(1)$ \\
\hline YI & -9.74 & $-0.08^{e}$ & $-0.43(1)$ & & $-0.71(1)$ & & & & & \\
\hline YII & $\cdots$ & -0.08 & ... & -0.23 & +0.09 & -0.4 & $-0.29(4)$ & $-0.45(2)$ & $-0.30(4)$ & $-0.20(5)$ \\
\hline ZrII & -9.35 & $-0.15^{f}$ & $-0.09(1)$ & $-0.51(1)$ & $-0.52(1)$ & $-0.60(2)$ & $-0.20(5)$ & $-0.45(3)$ & $-0.35(7)$ & $-0.41(8)$ \\
\hline BaIl & -9.74 & $0.00^{b}$ & $-0.39(1)$ & $-0.26(1)$ & $-0.16(1)$ & $-0.03(2)$ & $-0.29(2)$ & $-0.49(2)$ & +0.24 & $-0.43(2)$ \\
\hline LaII & -10.86 & $-0.05^{b}$ & $\ldots$ & 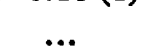 & $+0.05(1)$ & $\ldots$ & $+0.02(2)$ & $+0.15(1)$ & $-0.05(1)$ & $+0.16(3)$ \\
\hline$\overline{\mathrm{CeII}}$ & -10.35 & $-0.06^{b}$ & $-0.30(5)$ & $-0.07(2)$ & $-0.08(4)$ & $-0.07(2)$ & $-0.04(5)$ & $-0.20(6)$ & $-0.12(6)$ & $-0.19(6)$ \\
\hline NdII . & -10.53 & $+0.17 \mathrm{~d}$ & $+0.01(1)$ & $\ldots$ & $\ldots$ & $+0.47(2)$ & $+0.44(2)$ & $+0.17(2)$ & $\ldots$ & $+0.24(3)$ \\
\hline SmII & -11.04 & $-0.30 \mathrm{~d}$ & $-0.32(1)$ & $\ldots$ & $\ldots$ & ... & $+0.01(1)$ & $\ldots$ & $\ldots$ & $-0.26(1)$ \\
\hline
\end{tabular}

Note.- The sun is measured relative to $\mathrm{H}$, Canopus relative to the Sun, and the Cloud stars relative to Canopus.

a From Desikachary and Hearnshaw 1982.

b Differs by more than 0.1 dex from Desikachary and Hearnshaw 1982 (but less than 0.2 dex).

c From Blackwell et al. 1982.

d Differs by more than 0.2 dex from Desikachary and Hearnshaw 1982 (but less than 0.3 dex).

e Species not measured for Canopus by Lyubimkov and Boyarchuk 1982, therfore relative abundance from other species used.

f Differs by more than 0.3 dex from Desikachary and Hearnshaw 1982 (but less than 0.4 dex). 
TABLE 12

Average Relative Abundances in the Magellanic Clouds

\begin{tabular}{|c|c|c|c|c|}
\hline Element & {$[\mathrm{M} / \mathrm{H}]_{\mathrm{SMC}}$} & $\sigma_{\mathrm{SMC}}$ & {$[\mathrm{M} / \mathrm{H}]_{\mathrm{LMC}}$} & $\sigma_{\text {LMC }}$ \\
\hline $\begin{array}{l}\text { C I } \\
\text { Na I } \\
\text { Mg I } \\
\text { Si II } \\
\text { S I } \\
\text { Ca I } \\
\text { Ca II } \\
\text { Sc II } \\
\text { Ti I } \\
\text { Ti II } \\
\text { V I } \\
\text { V II } \\
\text { Cr I } \\
\text { Cr II } \\
\text { Mn I } \\
\text { Mn II } \\
\text { Fe I } \\
\text { Fe II } \\
\text { Ni I } \\
\text { Ni II } \\
\text { Cu I } \\
\mathrm{Zn} \mathrm{I} \\
\text { Sr II } \\
\text { Y I } \\
\text { Y II } \\
\text { Zr II } \\
\text { Ba II } \\
\text { La II } \\
\text { Ce II } \\
\text { Nd II } \\
\text { Sm II }\end{array}$ & $\begin{array}{c}-0.77 \\
-0.21 \\
-0.57 \\
\ldots \\
\ldots .0 \\
-0.64 \\
-0.71 \\
-0.59 \\
-0.58 \\
-0.35 \\
\ldots \\
-0.49 \\
-0.49 \\
-0.51 \\
.0 \\
-0.60 \\
-0.70 \\
-0.40 \\
-0.26 \\
-0.28 \\
\ldots \\
-1.60 \\
-0.45 \\
-0.64 \\
-0.68 \\
-0.89 \\
-0.58 \\
-0.48 \\
+0.02 \\
-0.04\end{array}$ & $\begin{array}{c}0.06 \\
\ldots .12 \\
\ldots \\
\ldots .19 \\
\ldots \\
0.16 \\
0.12 \\
0.11 \\
0.20 \\
\ldots \\
0.29 \\
0.19 \\
0.31 \\
\ldots \\
0.16 \\
0.19 \\
0.24 \\
\ldots \\
0.28 \\
\ldots \\
\ldots \\
0.34 \\
0.15 \\
0.20 \\
0.35 \\
0.12 \\
0.24 \\
\ldots \\
0.39\end{array}$ & $\begin{array}{r}-0.58 \\
+0.80 \\
-0.13 \\
+0.38 \\
-0.44 \\
-0.48 \\
.0 . \\
-0.43 \\
-0.23 \\
-0.23 \\
+0.16 \\
0.00 \\
-0.25 \\
-0.17 \\
-0.45 \\
-0.11 \\
-0.37 \\
-0.23 \\
-0.21 \\
\ldots \\
-0 . \\
-0.40 \\
-0.46 \\
-0.57 \\
-0.26 \\
-0.39 \\
-0.23 \\
-0.07 \\
-0.13 \\
+0.27 \\
-0.19\end{array}$ & $\begin{array}{c}0.18 \\
\ldots .13 \\
\ldots \\
\ldots .16 \\
\ldots .21 \\
0.13 \\
0.06 \\
0.34 \\
0.08 \\
0.18 \\
0.10 \\
0.31 \\
\ldots .17 \\
0.17 \\
0.09 \\
\ldots \\
\ldots \\
0.10 \\
0.58 \\
\ldots .18 \\
0.17 \\
0.24 \\
0.09 \\
0.09 \\
0.19 \\
0.18\end{array}$ \\
\hline
\end{tabular}


Figure 16 The average relative abundances for (a) the SMC, and (b) the LMC, plotted against the atomic number $Z$.The dashed line represents the average iron abundance of the Cloud while the solid line represents solar abundance. The open squares without error bars refer to elements represented by less than three stars. 

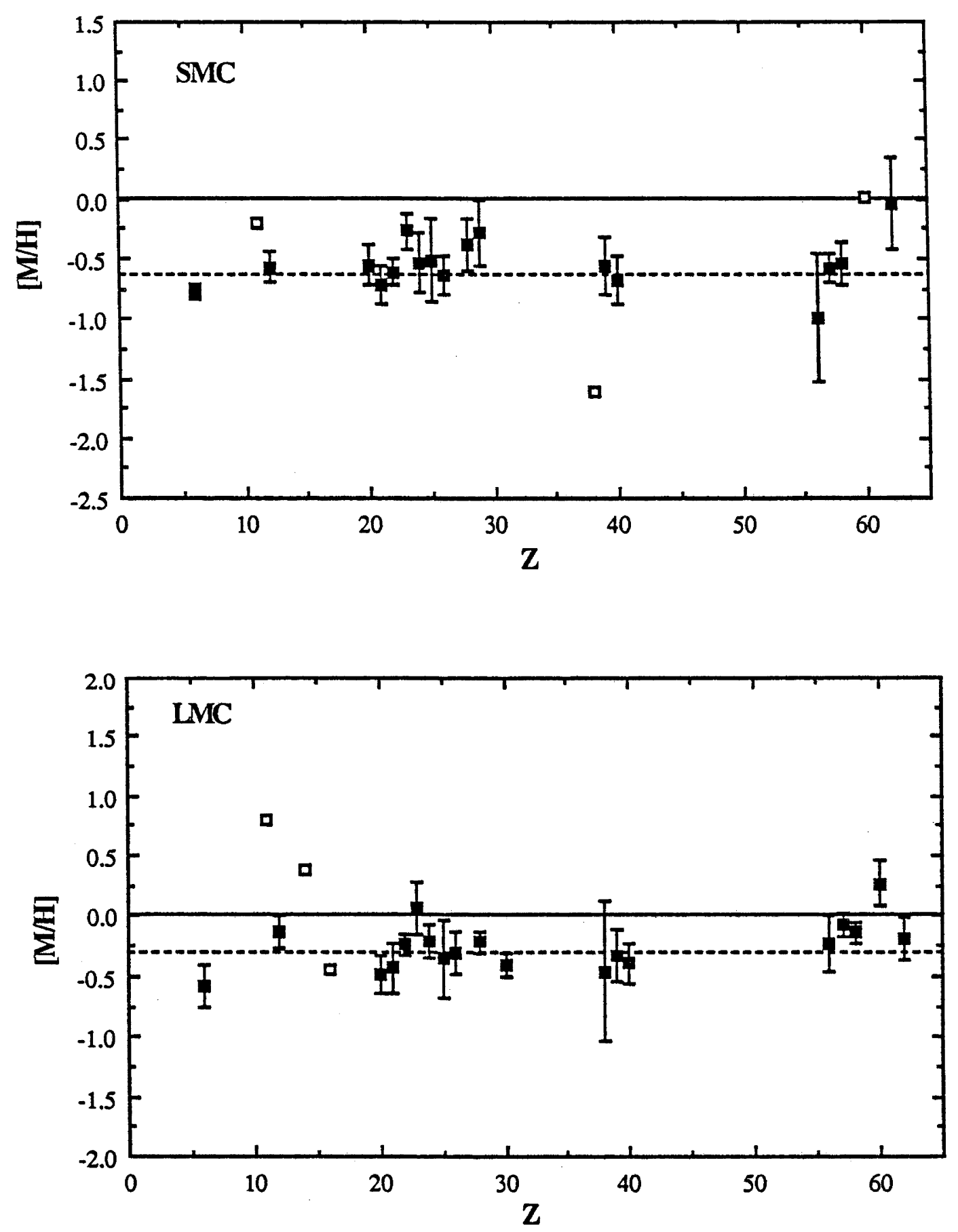
would be meaningless. The major result from our data is that the iron abundances for the Magellanic Clouds are

$$
\begin{aligned}
& {[\mathrm{Fe} / \mathrm{H}]_{S M C}=-0.65 \pm 0.2 \mathrm{dex},} \\
& {[\mathrm{Fe} / \mathrm{H}]_{\text {LMC }}=-0.30 \pm 0.2 \mathrm{dex} .}
\end{aligned}
$$

Both these values agree very well (see Tables 1 and 2 ) with the photometric metallicity estimates derived from Cepheids and with the abundance analysis of the three SMC supergiants by Spite, Spite, and François. (1988).

In general, for our own galaxy, the abundances of all heavy elements tend to track the abundance of $\mathrm{Fe}$, which we shall refer to here as the "normal" abundance. Elements having different abundances from the normal we refer to as being either "overabundant" or "underabundant." A casual inspection of Figure 16 reveals that most elements in the Clouds do seem to track the iron abundance. However, it would be more accurate to compare the abundances of the Cloud stars directly with the abundance of Canopus, since this would circumvent the errors in the abundances for Canopus that we added in and would have the advantage of comparing similar types of star directly. In this case, we define $[\mathrm{M} / \mathrm{H}]^{\text {stnd }}$ to be $\log N(\mathrm{M} / \mathrm{H})_{\mathrm{star}}-\log$ $N(\mathrm{M} / \mathrm{H})_{\text {standard }}$, the standard here being Canopus. Thus any differences in the abundances intrinsic to this type of star, would be canceled out. In order to take out most of the systematic errors due to inaccuracies in our derived physical parameters for the stars, we need to consider the ratio of an element with $\mathrm{Fe}$, which we expect from Galactic studies to give unity in every case (i.e. normal abundances). So here we define $[\mathrm{M} / \mathrm{Fe}]^{\text {stnd }}$ to be $\log N(\mathrm{M} / \mathrm{Fe})_{\text {star }}-\log N(\mathrm{M} / \mathrm{Fe})_{\text {standard, }}$ which is analogous to the absolute abundance difference defined above. A further reduction in systematic error results from ensuring that neutral species of an element are always ratioed with $\mathrm{Fe}$ I, while ionized species are ratioed with $\mathrm{Fe}$ II. This is important in our case since rigid Fe ionization balance has not been adhered to. These results are easily derived from Tables 11A and 11B, and are presented for 
each individual star in Figures $17 a$ and $17 b$. This shows the star-to-star variation in abundances, but it must be recalled that those abundances depending upon only one or two lines are more sensitive to observational and systematic errors than the others. Even so, there is a suggestion of a scatter in abundances from star-to-star of the order of 0.2 dex in excess of the observational errors. This may be confirmed, to some extent, by an example. Figures $2 a$ and $2 b$ show small sections of the spectra of two SMC stars, AV 401 and AV 79, respectively. As can be seen from Table 9, their physical parameters are very similar, and yet it is clear from Figure 2 that the lines in AV 79 are very much weaker than those in AV 401, especially in the case of the Ba II line at $4554 \AA$. Thus, we believe that this provides firm evidence for moderately incomplete mixing of the ISM in both of the Clouds.

Our preliminary analysis, (Russell, Bessell, and Dopita 1988a, b) of the star AV 79 indicated that it was anomalously underabundant, thus suggesting that there were cases of severely incomplete mixing. However, this star has been reanalyzed here, and as can be seen in Table 11A, the abundances are low, but when Fe, Ti, and $\mathrm{Cr}$ are considered together, it seems that this star is simply part of the lowabundance tail of the normal distribution of abundances in the SMC. In the preliminary analysis of this star, our estimate for the effective temperature was somewhat too low due to the presence of a close blue companion in the aperture during the photometry. However, the very low abundance measured for Ba still remains anomalous.

The averages of the abundances are shown in Figures $18 a$ and $18 b$. The most obvious result is that the abundances of the elements do track the abundance of iron very tightly when compared directly with the abundances for Canopus. To see if any of the small abundance differences from $\mathrm{Fe}$ are significant, it is essential for us to gather together any independent measures of elemental abundances in the stars of the Magellanic Clouds that are available in the literature. 
Figure 17 Individual stellar abundances over iron, relative to Canopus for $(a)$ the SMC, and $(b)$ the LMC. 

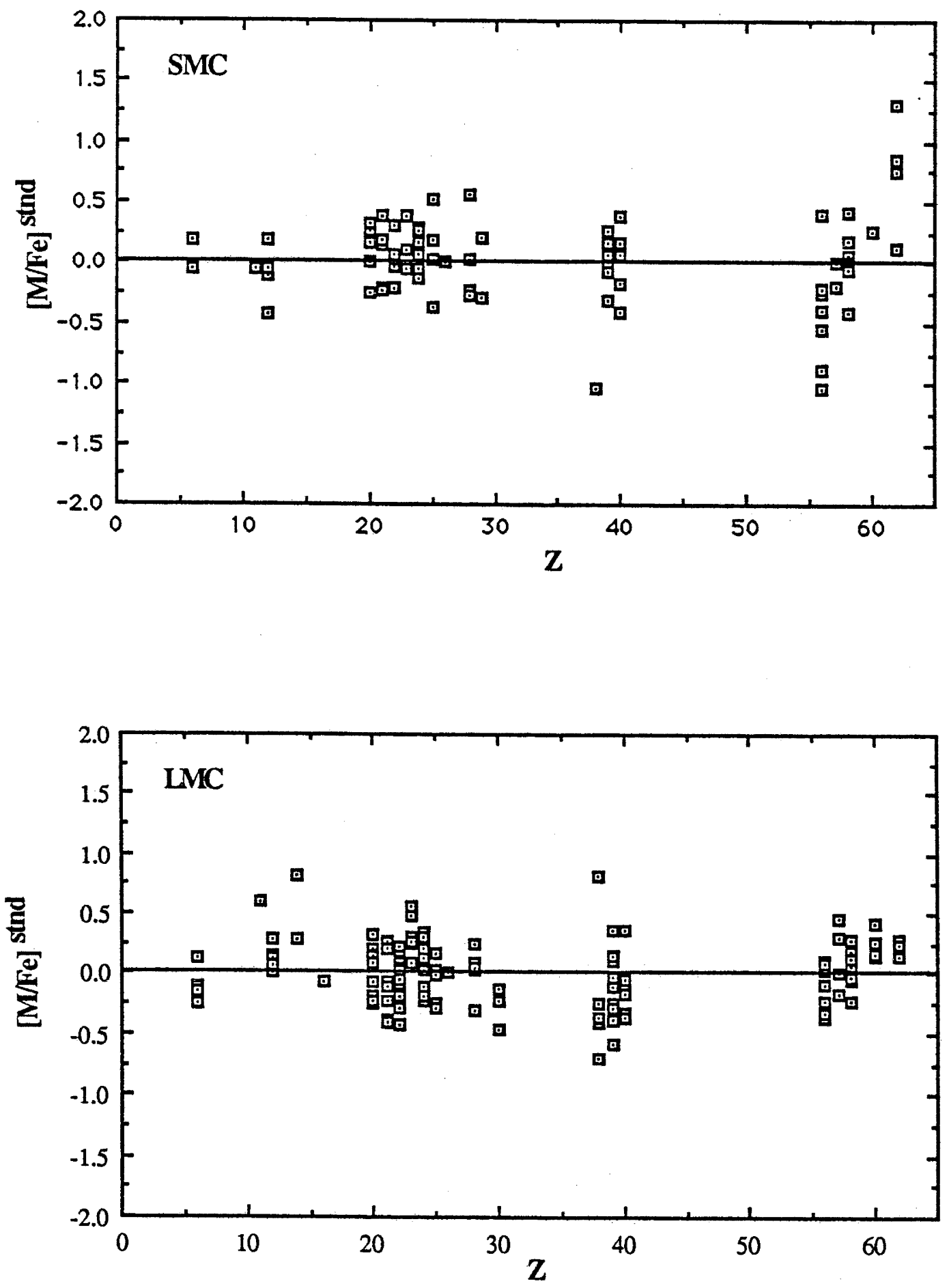
All the detailed abundance surveys that are listed in Tables 1 and 2 (except that of HD 6884 by Przybylski [1975], 'which appeared highly discrepant) have been summarized in Figures $19 a$ and $19 b$ for the SMC and LMC, respectively. In each case we have found the elemental abundances relative to a Galactic supergiant of similar type (usually observed by the authors at the same time) and have then derived the abundances relative to $\mathrm{Fe}$. This procedure minimizes the errors due to an imperfect knowledge of the physical characteristics of these stars. Our conclusions based on the sum total of our present knowledge of the abundances in the Clouds are presented in the following section.

\section{b) The Individual Elements}

\section{i) Carbon}

The Carbon abundance is not normally obtained from the spectra of $F$ supergiants because of the possibility of its abundance being modified by evolutionary processes in the star itself. However, the only type of modification that seems possible is a dilution of the atmospheric abundances by dredge up of CNO cycle products (Becker 1981, Becker and Cox 1982). The measured carbon abundances for the Galactic supergiants are under-abundant relative to iron, but reasonably uniform, as can be seen from Figure 12. The effect of reducing the metallicity to the levels found in the Clouds was estimated from the tables presented by Becker and Iben (1979) and was found, in the worst case, to result in an increase of the order of 0.1 dex in the carbon-to-iron ratio over that found in Canopus. There is a high degree of uniformity in the carbon abundances found for the Magellanic Cloud stars, as can be seen from the relatively small error bars for this element in Figures $18 a$ and $18 b$. The interesting thing is that compared with the Galactic supergiants, the Cloud carbon abundances relative to iron are entirely normal. This 
Figure 18 Average element to iron ratios for (a) the SMC, and (b) the LMC, relative to Canopus. The open squares without error bars refer to elements represented by less than three stars. 

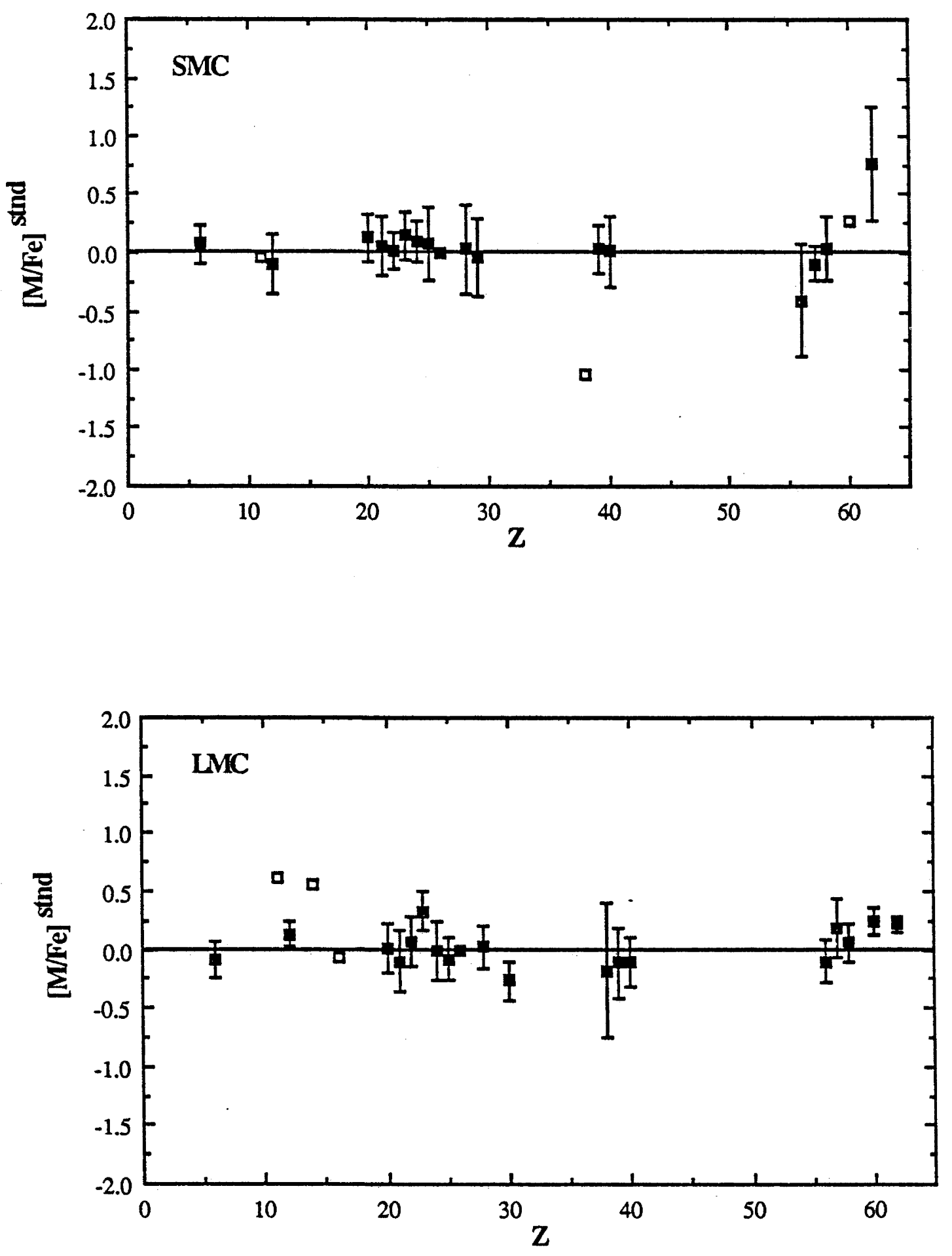
Figure 19 Average element to iron ratios for $(a)$ the SMC, and (b) the LMC, from the literature, relative to some Galactic standard. The open squares without error bars refer to elements represented by less than three stars. 

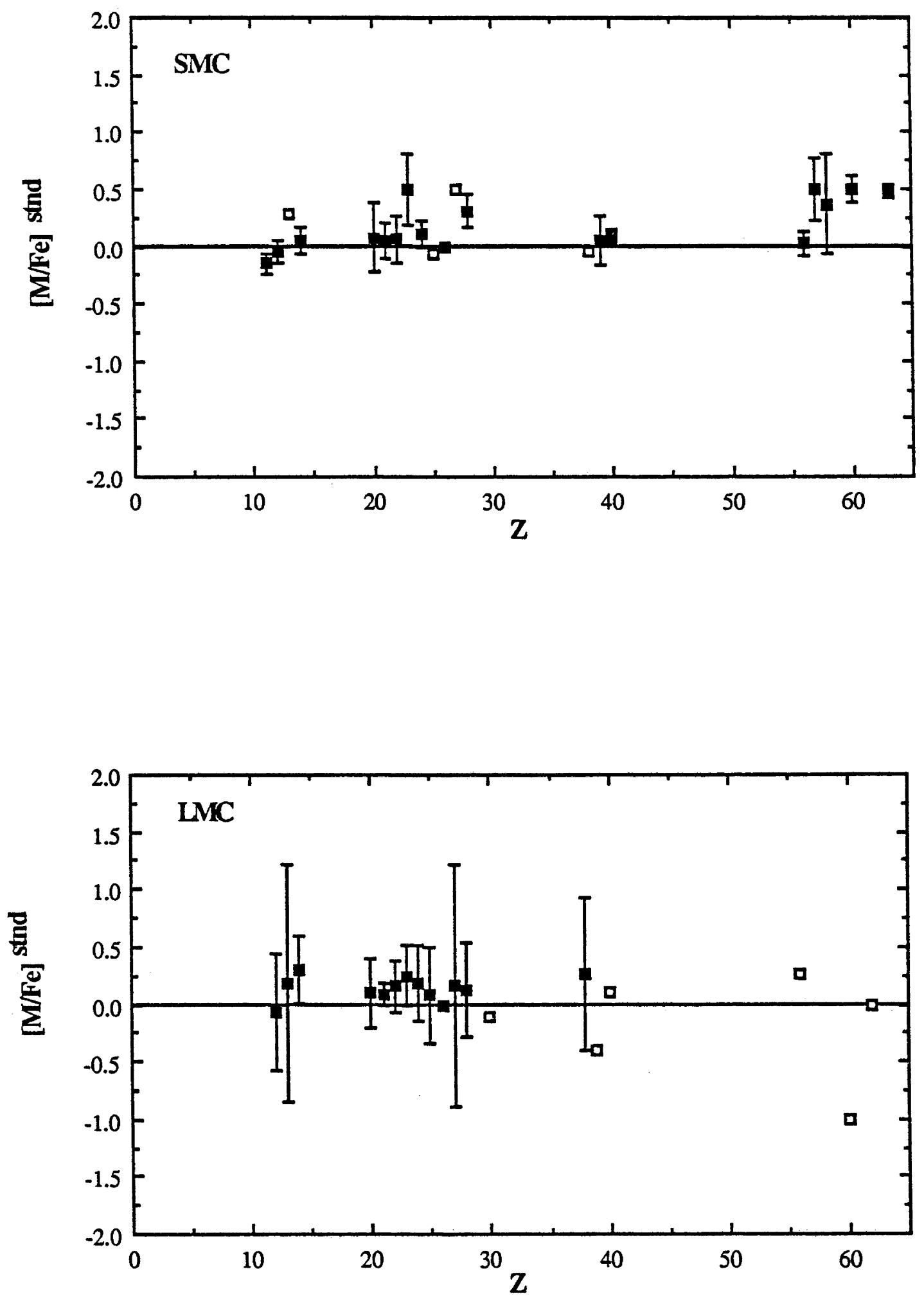
is in direct contrast to the results of UV spectroscopy of H II regions by Dufour, Shields, and Talbot (1982), using the IUE satellite. It must be remembered, however, that their method for deriving the carbon abundance suffers from grave uncertainties in the assumed fraction of carbon locked up in grain material and in the accuracy of the physical models used. Their study indicated that the carbon abundances in the Clouds were well down compared with the abundances of the heavier elements. Our explanation for this (Russell, Bessell, and Dopita 1988a, b) was that $\mathrm{Fe}$ and $\mathrm{C}$ are formed from stars of different mass and the carbon has not yet had time to build up to the same abundance as in our own galaxy. This new data, which indicates that the carbon abundances in the Clouds are normal, is far easier to understand, since it is in good accord with results from our own Galaxy, which indicate that carbon tracks iron at all metallicities (see Clegg 1977, Peterson 1978, Peterson and Sneden 1978, Barbuy 1981, and Clegg, Lambert, and Tomkin 1981). Further evidence supporting our results comes from two sources. Spite (private communication) informs us that their group has found the same small weakening of the $\mathrm{C} / \mathrm{Fe}$ ratio in their SMC stars as in Canopus. In addition, Reitermann et al. (1988) analyzed one near-main-sequence B-star in each of the Magellanic Clouds and found these stars to have normal or enhanced abundances, in particular for the elements $\mathrm{C}, \mathrm{N}$, and $\mathrm{O}$. These data seriously challenge the abundances derived from $\mathrm{H}$ II regions, which to date indicate that there are deficiencies of all three elements in both Clouds, which are severe in the case of $\mathrm{C}$ and $\mathrm{N}$.

One difficulty, however, must be born in mind in our derivation of [C/Fe]. The iron abundance is based upon low-excitation lines $(0-4 \mathrm{eV})$, while the carbon abundance is derived from high-excitation lines $(-7.7 \mathrm{eV})$. Thus, their direct comparison is quite sensitive to errors in the temperature structure of the model atmosphere. Fortunately, we would expect any errors in the temperature structure of the Cloud stars to be present in the Galactic stars as well, since they are of such 
similar types. Thus, the differential analysis should yield reliable results. These same comments must also be applied to the abundance ratios of the elements silicon and sulphur.

\section{ii) Sodium}

Sodium appears to be somewhat over-abundant relative to iron in the LMC, although it is of normal abundance in the SMC, relative to the Galactic supergiants. Both of these results are based on one line in one star each and are therefore not very reliable. Evidence from the literature is lacking for the LMC, but there is quite strong evidence from the literature that the sodium abundance is slightly underabundant in the SMC. It should be noted that the sodium abundance in the Galactic supergiants is already quite high (see Figures 12 and 13). This is not simply a nonLTE effect as commonly supposed (see discussion in Spite, Spite, and François (1988) and references therein), but is more probably due to the evolution of the stars themselves (see Lyubimkov and Boyarchuk (1982)). Therefore any variation in the general overabundance of sodium in the Cloud supergiants, may simply be reflecting internal processes within the stars, rather than the composition of the ISM.

\section{iii) Silicon and the $\alpha$-Elements}

Silicon is found by us to be somewhat overabundant relative to iron in the LMC, while no measure is available for the SMC. This result is based on slightly better evidence than for the case of sodium, as we have measurements for two stars, one of which has two lines. Evidence from the literature also points to some degree of overabundance in the LMC, but normal abundance in the SMC.

Silicon is one of the $\alpha$-elements $(\mathrm{Mg}, \mathrm{Si}, \mathrm{Ca}, \mathrm{Ti})$ primarily synthesized during explosive oxygen and silicon burning (Cameron 1973, Trimble 1975) through 
addition of $\alpha$-particles to lighter seed nuclei. There is strong evidence in the literature (see, for example, Luck and Bond 1985; Nissen, Edvardsson, and Gustafsson 1985; Abia et al. 1988; Andersen et al. 1988) that these elements behave in a similar manner to oxygen. However, analyses of $\mathrm{H} \mathrm{II}$ regions in the literature (see, for example, Dufour 1984, and references therein) indicate that oxygen is underabundant in the Clouds (compared with Galactic stars of the same metallicity). If this were the case, we would expect to see some reflection of this in the abundances of the $\alpha$-elements (especially in the SMC). No such trend is found, which is further evidence in support of the conclusions of Reitermann et al. (1988) that oxygen is of normal abundance in the Clouds.

\section{iv) Vanadium}

We find vanadium of normal abundance relative to iron in the SMC, but somewhat overabundant in the LMC. Evidence from the literature supports this overabundance in the LMC and suggests also an overabundance in the SMC. Our conclusion for the SMC is based on only one line in each of four stars and is therefore open to some doubts. However, our abundance for vanadium in the LMC is based on the results from five stars, each of which has between two and four contributing lines. This, then, does not seem to be so easily discarded as observational error.

Vanadium is an odd, iron-peak element and as such should suffer to some degree from the odd-even effect (Arnett 1971), resulting in a variation in the abundance relative to iron as a function of the metallicity. Unfortunately, the expected variation with decreasing metallicity is to lower abundances. Some other effect may thus be required to account for the observed overabundances. 
v) Zinc

Zinc is produced mainly by the same nuclear statistical equilibrium process that is responsible for production of the iron peak elements, and as such, is not expected to stray far from the normal abundance. It is not possible to comment upon the SMC, since there are no measures available, either from our own work, or from the literature. Our results for the LMC indicate that it is somewhat underabundant relative to iron. This, however, is based on just three stars with one line measured in each and, as such, cannot be relied upon too heavily. Indeed the evidence from the literature indicates a normal abundance for this element.

\section{vi) Strontium}

The massive underabundance in strontium evident in the SMC from our Figure $18 a$, is derived from only one line in only one star. The fact that the other two elements in the light neutron capture group, $\mathrm{Y}$ and $\mathrm{Zr}$, appear normal argues that this point is discrepant. Further evidence for this comes from the normal abundance of this element reported in the literature (see Figure 19a), and from the normal abundance that we obtain for this element in the LMC. Thus, until further evidence on this question is forthcoming, we must assume that strontium has a normal abundance in the SMC as well as the LMC.

vii) The Heavy Neutron-Capture Elements

For both the SMC and the LMC, we find an indication of an overabundance in the heavy neutron-capture elements, $\mathrm{Nd}$ and $\mathrm{Sm}$. A similar trend was found by Spite, Spite, and François (1988) for their three stars in the SMC. Indeed their results go further and indicate that the almost pure $r$-process element $\mathrm{Eu}$, is also overabundant. It should be noted that the element $\mathrm{Sm}$ is largely (63\% according to Cameron (1982a) an $r$-process element, and we found it over-abundant by a similar 
amount to that found by Spite, Spite, and François (1988) for Eu. There is, however, one measurement in the literature (Fig. 19b) for the LMC that indicates a strong underabundance for $\mathrm{Nd}$. This result was from an extreme star with a very crowded spectrum, and the results are not considered very reliable, even by the author. According to Cameron (1982a), only 32\% of this element is due to the $r$ process, however there is some evidence in the literature (Luck and Bond 1985, Bond and Luck 1988, Gilroy et al. 1988) that production of this element may be dominated by the $r$-process. This is intriguing, since evidence from our own galaxy (see Spite and Spite 1978) suggests that $r$-process elements, being of primary origin, should track iron back to very low metallicities, much lower than the metallicities of the Clouds.

Again, caution must be exercised in interpreting the data from the rare earths due to their somewhat lower excitation potential in comparison to $\mathrm{Fe}$ II. However, inspection of Figure 14a reveals that in a typical case at least, there is no systematic difference in the abundances derived from low excitation potentials (as low as $0 \mathrm{eV}$ ) and high excitation potentials for Fe I. In addition, we stress once more that this is a differential analysis and, as such, most systematic errors should cancel out with similar errors in sufficiently similar Galactic counterparts.

No firm conclusions can as yet be drawn, but there is an increasing amount of evidence accumulating for the reality of an overabundance of the heavy elements in both Clouds. If true, then we must look at differences in the star formation or nucleosynthetic processes between our Galaxy and the Clouds in order to explain it. This shall be discussed in the light of all the available evidence in Paper III of this series. 


\section{THE SMC STAR AV 369}

As mentioned previously in this paper, the SMC star AV 369 has been studied both by Foy (1981) and by Spite, Spite, and François (1988). Figure 1 shows that this star (the two points connected by a dotted line) is very bright, more than a magnitude brighter than anything we have considered so far in our survey. This means that the physical parameters will be harder to measure, and the classical assumptions made in our analysis will start to break down. This star is already so extreme that there appears, from the observation of strong emission in the wings of the H $\alpha$ line by Spite, Spite, and François (1988), to be some degree of mass loss . Thus, the assumption of a hydrostatic atmosphere is no longer fully justified.

First let us consider the spectral types for this star as reported in the literature:

$\begin{array}{lll}\text { Sanduleak (1968) } & -- & \text { G5 I, } \\ \text { Dachs (1970). } & -- & \text { F8, } \\ \text { Azzopardi and Vigneau (1975). } & -- & \text { G0, } \\ \text { Dubois, Jaschek, and Jaschek (1977). } & -- & \text { F7 Ia from Ca lines, } \\ & -- & \text { F2 Ia from FeIl lines, } \\ \text { Humphreys (1983). } & -- & \text { F5 Ia. }\end{array}$

The temperature for this star seems to be equally debatable. Foy (1981), in his analysis of the star AV 369, determined the effective temperature to be $5800 \mathrm{~K}$, from the fitting of models to the wings of the $H \beta$ line. This is a very imprecise method of determining the temperature, especially for this type of star. Parsons (1971) found that if he modeled the wings of the H $\alpha$ and $H \gamma$ lines for the stars $\alpha$ Per (F5 Ib) and $\beta$ Aqr (G0 Ib), he could obtain a good fit, but only at the expense of having a very poor fit for the cores of these lines. As a result he concluded that this method of determining the temperature was not reliable. Further, as mentioned previously, Spite, Spite, and François (1988) found emission in the wings of the 
$\mathrm{H} \alpha$ line of AV 369 and thus strongly suspected faint emission in the wings of the $\mathrm{H} \beta$ line that would distort any estimation of temperature made from consideration of this line. Instead, Spite, Spite, and François (1988) decided to determine the temperature from broad-band photometry alone and arrived at a temperature of 6450 $\mathrm{K}$ for AV 369. This agrees well with our calculation of the temperature from Strömgren and broad-band photometry published in the literature. Osmer (1973) determined $b-y$ to be 0.35 , and $c_{1}$ to be 1.23 , while Grieve and Madore (1986a) determined the reddening to be $E(b-y)=0.14$. Thus the intrinsic values for $b-y$ and $c_{1}$ are 0.21 and 1.20, respectively. From the models of Lester, Gray, and Kurucz (1986) we determined the temperature to be $6540 \mathrm{~K}$ and $\log g$ to be 1.79 . Although we obtain good agreement in temperature, the evolutionary gravity (see Fig. 1) is closer to $0.8 \mathrm{dex}$, which is in strong support of the estimate of $\log g=0.5$ by Spite, Spite, and François (1988) in their non-LTE analysis of the star. It is therefore probable that the value for $c_{1}$ quoted in the literature is in error because of the inclusion of a close companion during the photometry.

Thus, while we support the conclusions of Spite, Spite, and François on the physical parameters of this star, we find Foy's estimate of the temperature far too low. In addition, we modeled those lines published by Foy that were in common with our own line list for Canopus, and we found a good measure of the turbulent velocity for the ionized species of $\xi_{I I}=12 \mathrm{~km} \mathrm{~s}^{-1}$. We could not make any firm conclusions on the neutral turbulent velocity, because of the poor quality of the data, but from our Figure 15 we would prefer the value $\xi_{I}=7 \mathrm{~km} \mathrm{~s}^{-1}$, derived by Spite, Spite, and François to the low value derived by Foy of $\xi_{I}=3.8 \mathrm{~km} \mathrm{~s}^{-1}$. 


\section{THE SMC STAR AV 121}

The SMC star AV 121 is almost as bright as the previous star we considered, AV 369 (see Fig. 1 - the star closest to AV 369 in the diagram is AV 121). Thus we might expect the same problems we had for AV 369. There has been no study made of the $\mathrm{H} \alpha$ line, so we cannot say whether the wings are in emission or whether there is substantial mass loss in progress. We might assume, however, that the assumption of hydrostatic equilibrium is no longer accurate.

Let us now consider the spectral types quoted in the literature:

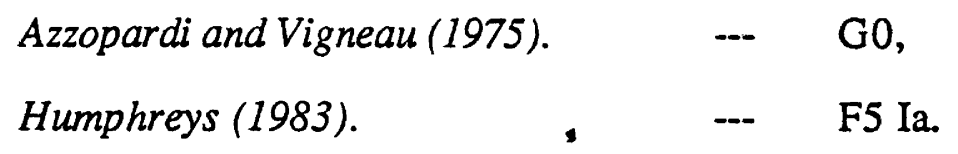

In this case, there was a comment by Grieve and Madore (1986a) to the effect that their photometry indicated that AV 121 must be later than F5 at least. An effective temperature of $5930 \mathrm{~K}$ was determined from the wings of the HB line by Thévenin and Foy (1986). For reasons already stated for the case of AV 369, we were not confident that this was a reliable temperature. However, our own photometry (Tables 4, 5, and 6) indicated an effective temperature of $5780 \mathrm{~K}$ for this star, well within the errors of the value derived by Thévenin and Foy and in close agreement with the temperature derived from the spectral type suggested by Azzopardi and Vigneau (1975). Our estimate for the gravity of $\log g=1.35$ is also close to that derived by Thévenin and Foy of $\log g=1.3$. Unfortunately, the evolutionary gravity (see Fig. 1) is closer to $\log g=0.7$, thus lowering our confidence in the other estimates of gravity. The uncertainty in the physical parameters of this star prompted us to exclude it from the general survey and to consider it separately. 
We took one echelle spectrum of this star (see Table 7), and from the lines we had in common with Thévenin and Foy we showed that there was almost no systematic error $(<0.03$ dex) between our two sets of measurements and a random error of 0.14 dex. Our equivalent widths (see Table 8) for AV 121 were combined with those from Thévenin and Foy that were in common with the lines we had measured for Canopus (and could thus be related directly to that standard). From this composite set, we derived the abundances for this star using the same temperature and gravity as used by Thévenin and Foy. We found, however, that their value for the turbulent velocity of $\xi_{T}=3.5 \mathrm{~km} \mathrm{~s}^{-1}$ was far too low. We obtained reasonably good measures for both neutral and ionized species of $\xi_{\mathrm{I}}=\xi_{\text {II }}$ $=8.0 \mathrm{~km} \mathrm{~s}^{-1}$. Using this microturbulence and the effective temperature and gravity used by Thévenin and Foy (to facilitate comparisons), we analyzed the abundances of AV 121 and we present them graphically in Figure 20. Here we see that the rather high iron abundance of $[\mathrm{Fe} / \mathrm{H}]=-0.4$, derived by Thévenin and Foy (see Table 1 ), has now reduced to the mean iron abundance for the SMC as a whole (the dotted line in Fig. 20).

There is a large scatter apparent in the abundances of the elements other than iron, but most of these are represented by only one or at most two lines and should therefore not be relied upon. The remaining scatter in the elements represented by more than two lines is probably due to the poor quality of the data, combined with the poorly determined physical parameters of the star. 
Figure 20 Relative abundances for the star AV 121, derived from both our own work and that of Thévenin and Foy (1986). The open squares without error bars refer to elements represented in our combined spectra by less than three lines. 


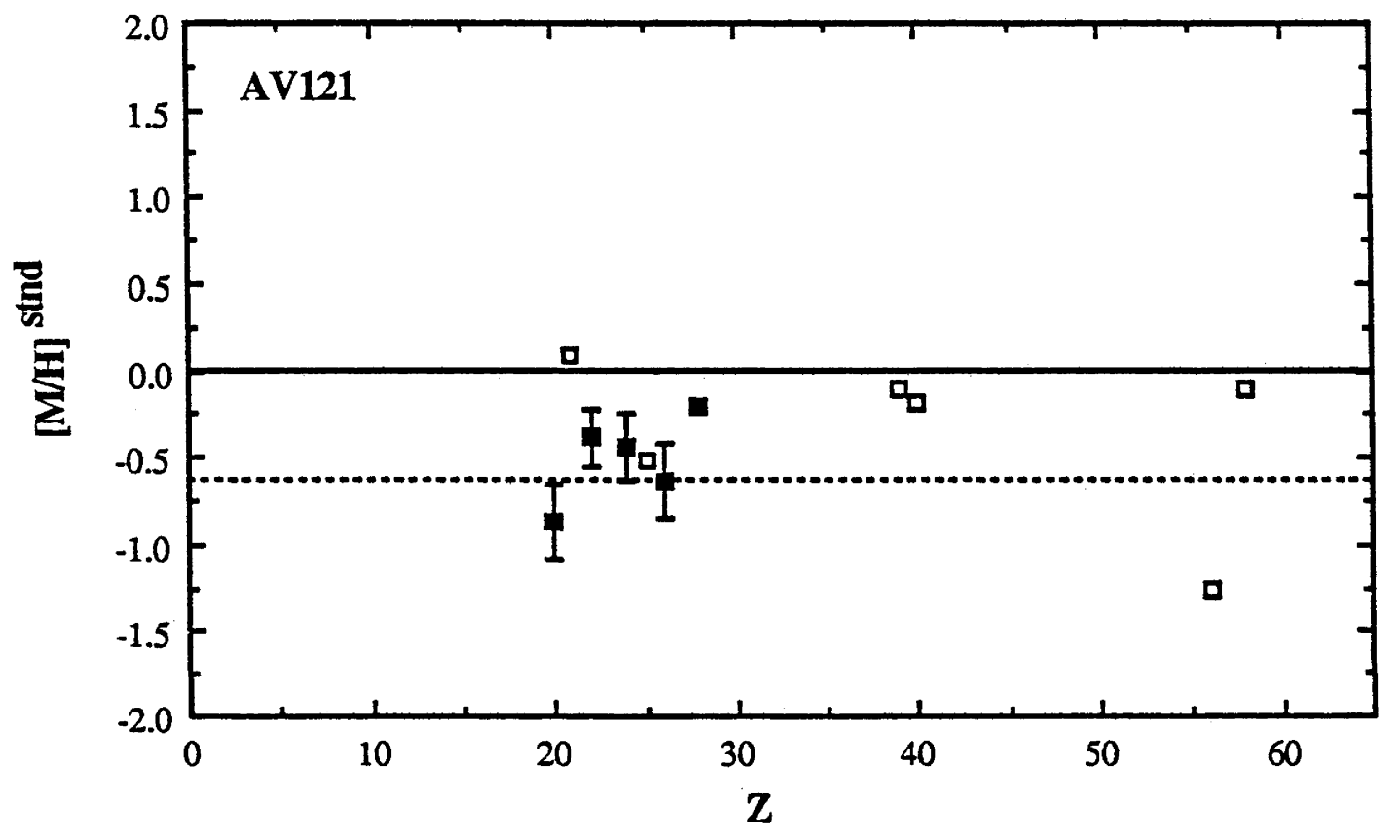




\section{SUMMARY}

We have made a detailed analysis of eight low luminosity $\mathrm{F}$ supergiants in both the SMC and the LMC and have been able to derive the abundances for over 20 elements in a consistent manner in both Clouds. Our Strömgren and broad-band $B V R I_{C}$ photometry of all 16 stars (plus three more in the LMC) allowed us to derive the reddening and the photometric temperatures and gravities for these stars. We noted that the Strömgren index, $m_{1}$, is no longer a useful indicator of metallicity for these types of stars and suggested that the large microturbulent velocities encountered in $\mathrm{F}$ supergiants may be responsible.

Analysis of our spectroscopic data revealed:

1. The microturbulent velocities of the Cloud stars are very similar to those of Galactic F supergiants.

2. The iron abundance of the $S M C$ is $[\mathrm{Fe} / \mathrm{H}]=-0.65 \pm 0.2 \mathrm{dex}$.

3. The iron abundance of the $L M C$ is $[\mathrm{Fe} / \mathrm{H}]=-0.30 \pm 0.2$ dex.

4. Most elements studied are as depleted as iron relative to Canopus.

5. The star-to-star variation in iron abundance in both Clouds is within the observational errors. There is, however, a suggestion of a scatter in abundances in the Clouds of perhaps $\sim 0.2$ dex in addition to the observational uncertainties. This would be an indication of moderately incomplete mixing of the ISM in each Cloud. The SMC star AV 79, is now believed to lie on the low-abundance tail of the normal scatter in abundances and is not anomalously underabundant as we concluded from our previous work.

6. The Carbon abundance in both Clouds is entirely normal compared to Canopus, not dramatically underabundant as suggested in the literature. 
7. Vanadium abundance is rather high in the LMC, a conclusion having some support from the literature.

8. The heavy neutron-capture elements, $\mathrm{Nd}$ and Sm, appear to be significantly overabundant in both Clouds. This substantiates a similar trend found by Spite, Spite, and François (1988) for the three SMC stars they studied.

9. Both the SMC G0 Ia supergiants AV 369 and AV 121 have problems in the determination of their physical parameters. We derived much higher microturbulent velocities for these stars than was derived in the previous analyses of the two stars by Foy (1981), and Thévenin and Foy (1986), respectively, thus explaining the rather high estimates for the metallicities of these stars derived by these authors.

Thanks to Mike Dopita for his welcome comments and ready insight into the overall picture developing through this work. Helpful discussions were also had with many people, in particular with Peter Wood. Thanks to all those who have helped in making the observations and to the Directors and staff of both Mount Stromlo and Siding Spring Observatories and the Anglo-Australian Observatory for the use of their facilities. The Australian Time Allocation Committee has been generous in their support for this project and are gratefully acknowledged. Finally, one of us (S. C. R.) would like to acknowledge the financial support received from a Commonwealth Postgraduate Scholarship during the course of this work. 


\section{REFERENCES}

Abia, C., Rebolo, R., Beckman, J. E., Crivellari, L., and Vila, B. 1988, in IAU Symposium 132, The impact of very high S/N Spectroscopy on Stellar Physics, ed. G. Cayrel and M. Spite (Dordrecht: Reidel), p. 421.

Allen, M. S. 1976, Pub. A.S.P., 88, 338.

Andersen, T., Edvardsson, B., Gustafsson, B., and Nissen, P. E. 1988, in IAU Symposium 132, The impact of very high S/N Spectroscopy on Stellar Physics, ed. G. Cayrel and M. Spite (Dordrecht: Reidel), p. 441.

Andersen, T., Poulsen, O., Ramanijan, P. S. and Petrakiev Petkov 1975, Sol. Phys., 44, 257.

Ardeberg, A., Brunet, J. -P., Maurice, E. and Prévot, L. 1972, Astr. Ap. Suppl., 6, 249.

Arnesen, A., Bengtsson, A., Hallin, R. and Noreland, T. 1977, J. Phys. B, 10, 565.

Arnett, W. D. 1971, Ap. J., 166, 153.

Azzopardi, M. and Vigneau, J. 1975, Astr. Ap. Suppl., 22, 285.

Barbuy, B. 1981, Astr. Ap., 101, 365.

Basinski, J. M., Bok, B. J., and Bok, P. F. 1967, M.N.R.A.S., 137, 55.

Becker, S. A. 1981, Ap. J. Suppl., 45, 475.

Becker, S. A. and Cox, A. N. 1982, Ap. J., 260, 707.

Becker, S. A. and Iben, I., Jr. 1979, Ap. J., 232, 831.

Beckers, J. M., Bridges, C. A., and Gilliam, L. B. 1976, A High Resolution Spectral Atlas of the Solar Irradiance From 380 to 700 Nanometers, (Hanscom, MA: Air Force Geophysics Laboratory).

Bessell, M. S. and Norris, J. 1984, Ap. J., 285, 622. 
Biemont, E., Grevesse, N., Hannaford, P. and Lowe, R. M. 1981, Ap. J.2 248, 867.

Blackwell, D. E., Ibbetson, P. A., Petford, A. D. and Shallis, M. J. 1979, M.N.R.A.S., 186, 633.

Blackwell, D. E., Menon, L. R. and Petford, A. D. 1984, M.N.R.A.S., 207, 533.

Blackwell, D. E., Menon, L. R., Petford, A. D. and Shallis, M. J. 1982, M.N.R.A.S., 201, 611.

Blackwell, D. E., Shallis, M. J. and Simmons, G. J. 1980, Astr. Ap., 81, 340. 1982, M.N.R.A.S., 199, 37.

Blanco, V. M., Demers, G. G., Douglass, G. G., and Fitzgerald, M. P. 1970, Pub. US Naval Obs., No. 21.

Boksenberg, A. and Burgess, D. 1972, Electronics and Electron Phys., 33B, 835.

Bond, H. E. and Luck, R. E. 1988, in IAU Symposium 132, The impact of very high S/N Spectroscopy on Stellar Physics, ed. G. Cayrel and M. Spite (Dordrecht: Reidel), p. 477.

Booth, A. J., Blackwell, D. E. and Petford, A. D. 1984, M.N.R.A.S., 208, 147.

Boyarchuk, A. A. and Lyubimkov, L. S. 1982, Astrofizika, 18, 375. . 1984, Astrofizika, 20, 85.

Boyarchuk, A. A., Lyubimkov, L. S., and Sakhibullin, N. A. 1984, Astrofizika, 22, 339 .

Bridges, J. M. and Kornblith, R. L. 1974, Ap. J., 192, 793.

Brunet, J. P. 1975, Astr. Ap., 43, 345.

Caldwell, J. A. R. and Coulson, I. M. 1985, M.N.R.A.S., 212, 879. 1986, M.N.R.A.S., 218, 223.

Cameron, A. G. W. 1973, Space Sci. Rev., 15, 121. . 1982a, Ap. Space Sci., 82, 123. 
Cameron, A. G. W. 1982b, in Essays in Nuclear Astrophysics, ed. C. A. Barnes, D. D. Clayton, and D. N. Schraam (Cambridge University Press), p.23.

Carney, B. W., Janes, K. A. and Flower, P. J. 1985, A. J., 90, 1196.

Castley, J. C. and Watson, R. D. 1980, Astr. Ap. Suppl., 41, 397.

Clegg, R. E. S. 1977, M.N.R.A.S., 181, 1.

Clegg, R. E. S., Lambert, D. L., and Tomkin, J. 1981, Ap. J., 250, 262.

Cohen, J. G. 1978, Ap. J., 223, 487.

Corliss, C. H. and Bozman, W. R. 1962, NBS Monog., No. 53.

Cousins, A. W. 1980, South Africa Astr. Obs. Circ. 1, 234.

Crampton, D. 1979, Ap. J., 230, 717.

Crampton, D. and Greasley, J. 1982, Pub. A.S.P., 94, 31.

Crawford, D. L. 1975, Dudley Obs. Rept., 9, 17.

Crawford, D. L., Barnes, J. V., and Golson. J. C. 1971, A. J., 76, 1058.

Dachs, J. 1970, Astr. Ap., 9, 95.

Delbouille, L., Neven, L., and Roland, G. 1973, Photometric Atlas of the Solar Spectrum from $\lambda 3000$ to $\lambda 10,000$ (Liège: Institut d'Astrophysique).

Desikachary, K. and Hearnshaw, J. B. 1982, M.N.R.A.S., 201, 707.

Dickens, R. J. and Powell, A. L. T., M.N.R.A.S., 161, 249.

Dopita, M. A. 1977, Ap. J. Supp., 33, 437.

Dopita, M. A., Binette, L., D'Odorico, S., and Benvenuti, P. 1984, Ap. J., 276, 653.

Dubois, P., Jaschek, M., and Jaschek, C. 1977, Astr. Ap., 60, 205.

Dufour, R. J. 1984, in IAU Symposium 108, Structure and Evolution of the Magellanic Clouds ed. S. van den Bergh and K. S. de Boer, (Dordrecht: Reidel), p. 353.

Dunham, T. Jr. 1929, Princeton Uni. Contrib., No. 9.

Fehrenbach, Ch. and Duflot, M. 1970, Astr. Ap. Special Suppl. Ser., 1. 
Fernie, J. D. 1983, Ap. J. Suppl., 52, 7.

Florsch, A. 1972, Pub. Obs. Astr. Strasbourg, $2,1$.

Foy, R. 1981, Astr. Ap., 103, 135.

François, P. 1986, Astr. Ap., 160, 264.

Fry, M. A. and Aller, L. H. 1975, Ap. J. Suppl., 29, 55.

Fuhr, J. R. 1988, J. Phys. Chem. Ref. Data, in press.

Fuhr, J. R., Martin, G. A., Wiese, W. L. and Younger, S. M. 1981, J. Phys. Chem. Ref. Data, 10, 305.

Froese-Fischer, C. 1975, Can. J. Phys., 53, 184.

Gilroy, K. K., Sneden, C., Pilachowski, C. A., and Cowan, J. J. 1988, Ap. J., 327, 298..

Graham, J. A., and Slettebak, A. 1973, A. J., 78, 295.

Gratton, R. G. 1982, Ap. J., 257, 640.

Gratton, R. G. and Sneden, C. 1987, Astr. Ap., 178, 179.

Grieve, G. R. and Madore, B. 1986a, Ap. J. Suppl., 62, 427. 1986b, Ap. J. Suppl., 62, 451.

Griffin, R. and Griffin, R. 1979, A Photometric Atlas of the Spectrum of_Procyon (Ilkley: Scolar).

Grønbech, B. and Olsen, E. H., 1976, Astr. Ap. Suppl., 25, 213.

Harris, H. C. 1981, A. J., 86, 1192.

Hearnshaw, J. B. and K. Desikachary, K. 1982, M.N.R.A.S., 198, 311.

Heise, H. 1974, A. Ap., 34, 275.

Höflich, P. 1988, Proc. Astr. Soc. Australia, in press.

Holweger, H. 1972, Solar Phys., 25, 14.

Humphreys, R. M. 1983, Ap. J., 265, 176.

Isserstedt, J. 1975, Astr. Ap., 41, 175.

Isserstedt, J. 1977, Astr. Ap., 54, 553. 
Johnson, H. L. 1966, Ann. Rev. Astr. Ap., 4, 193.

Kato, K. and Sadakane, K. 1982, Astr. Ap., 113, 135.

Kilkenny, D., and Hill, P. W. 1975, M.N.R.A.S., 173, 625.

Kilkenny, D., Hill, P. W. and Brown, A. 1977, M.N.R.A.S., 178, 123.

Krueger, T. K., Aller, L. H., Ross, J. and Czyzak, S. J. 1968, Ap. J., 152, 765.

Kurucz, R. L. 1970, Smithsonian Ap. Obs. Spec. Rept. No. 309. . 1979, Ap. J. Suppl., 40, 1. 1981, Smithsonian Ap. Obs. Spec. Rept., No. 390.

Kurucz, R. L. and Peytremann, E. 1975, Smithsonian Ap. Obs. Spec. Rept., No. 362.

Lambert, D. L., Mallia, E. A. and Warner, B. 1969, M.N.R.A.S., 142, 71.

Lester, J. B., Gray, R. O., and Kurucz, R. L. 1986, Ap. J. Suppl., 61, 509.

Lester, J. B., Lane, M. C., and Kurucz, R. L. 1982, Ap. J., 260, 272.

Luck, R. E. 1979, Ap. J., 232, 797.

Luck, R. E. and Bond, H. E. 1985, Ap. J., 292, 559.

Luck, R. E. and Lambert, D. L. 1981, Ap. J., 245, 1018.

Lyubimkov, L. S. and Boyarchuk, A. A. 1982, Astrofizika, 18, 596. 1983, Astrofizika, 19, 683.

Maeder, A. 1981, Astr. Ap., 102, 401.

Magain, P. 1985, Astr. Ap., 146, 95.

May, M., Richter, J. and Wichelmann, J. 1974, Astr. Ap. Suppl., 18, 405.

McNamara, D. H. and Feltz Jr., K. A. 1980, Pub. A.S.P., 92, 587.

Miller, M. H., Wilkerson, T. D., and Roig, R. A. 1974, Phys.Rev., A, 9, 2312.

Moity, J. 1983, Astr. Ap. Suppl., 52, 37.

Moore, C. E., Minnaert, M. G., and Houtgast, J. 1966, NBS Monog., No. 61.

Morgan, W. W., Harris, D. L., and Johnson, H. L. 1953, Ap. J., 118, 92. 
Nissen, P. E., Edvardsson, B., and Gustafsson, B. 1985, in ESO Conf. Proc. 21, Production and Distribution of $C, N, O$ Elements, ed. I. J. Danziger, F.

Matteucci, and K. Kjär (Garching: ESO), p. 131.

Olsen, E. H. 1983, Astr. Ap. Suppl., 54, 55.

Osmer, P. S. 1972, Ap. J. Suppl., 24, 255. 1973, Ap. J., 181, 327.

Pagel, B. E. J., Edmunds, M. G., Fosbury, R. A. E., and Webster, B. L. 1978, M.N.R.A.S., 184, 569.

Parsons, S. B. 1971, in Proc. Third Colloquium on Astrophysics, Colloquium on Supergiant Stars, ed. M. Hack (Trieste: Osservatorio Astronomico), p. 222.

Parsons, S. B. and Bouw, G. D. 1971, M.N.R.A.S., 152, 133.

Pel, J. W. 1983, in IAU Symposium 108, Structure and Evolution of the

Magellanic Clouds ed. S. van den Bergh and K. S. de Boer, (Dordrecht: Reidel), p. 169.

Pel, J. W., van Genderen, A. M., and Lub, J. 1981, Astr. Ap., 99, L1.

Peterson, R. C. 1978, Ap. J., 222, 181.

—. 1981, Ap. J., 244, 989.

Peterson, R. C. and Sneden, C. 1978, Ap. J., 225, 913.

Philip, A.G. D., and Philip, K. D. 1973, Ap. J., 179, 855.

Pilachowski, C. A., Wallerstein, G., and Leep, E. M. 1980, Ap J., 236, 508.

Przybylski, A. 1968, M.N.R.A.S., 139, 313.

. 1971, M.N.R.A.S., 152, 197. 1972, M.N.R.A.S., 159, 155.

. 1975, Royal Greenwich Obs. Bull., No. 182, 133.

. 1979, New Zealand J. Sci., 22, 393.

Reitermann, A., Stahl, O., Wolf, B., Baschek, B. and Scholz, M. 1988, talk given at the IAU General Assembly, (Baltimore). 
Relyea, L. J. and Kurucz, R. L. 1978, Ap. J. Suppl., 37, 45.

Rodgers, A. W., Harding, P., Bloxham, G., and Bessell, M. S. 1988, in IAU Symposium 132, The impact of very high S/N Spectroscopy on Stellar Physics, ed. G. Cayrel and M. Spite (Dordrecht: Reidel), p. 23.

Rousseau, J., Martin, N., Prévot, L., Rebeirot, E., Robin, A., and Brunet, J.P. 1977, Astr. Ap., 31, 243.

Russell, S. C., Bessell, M. S., and Dopita, M. A. 1987, Proc. Astr. Soc. Australia, 7,65 .

Russell, S. C., Bessell, M. S., and Dopita, M. A. 1988a, in Proc. NATO Advanced Study Institute, Galactic and Extragalactic Star Formation, ed. R. E. Pudritz and M. Fich eds. (Dordrecht: Kluwer), p. 601.

. 1988b, in IAU Symposium 132, The impact of very high S/N Spectroscopy on Stellar Physics, ed. G. Cayrel and M. Spite (Dordrecht: Reidel), p. 545.

Saffman, L. and Whaling, W. 1979, J. Quant. Spectrosc. Rad. Transf., 21, 93.

Sanduleak, N. 1968, A. J., 73, 246.

ـ 1969, Cerro Tololo Inter-American Obs. Contr., No. 89.

Schmid-Burgk, K. and Scholz, M. 1975, Astr. Ap., 41, 41.

Shobbrook, R. R. 1986, M.N.R.A.S., 219, 495.

Smith, H. A, 1980, A. J., 85, 848.

Smith, H. A. and Connolly, L. 1984, in IAU Symposium 108, Structure and Evolution of the Magellanic Clouds ed. S. van den Bergh and K. S. de Boer, (Dordrecht: Reidel), p. 223.

Sneden, C. and Parthasarathy, M. 1983, Ap. J., 267, 757.

Spite, M., Cayrel, R., François, P., Richtler, T., and Spite, F. 1986, Astr. Ap., $168,197$.

Spite, M. and Spite, F. 1978, Astr. Ap., 67, 23.

Spite, F., Spite, M., and François, P. 1988, preprint. 
Stapinski, T. E., Rodgers, A. W., and Ellis, M. J. 1981, Pub. A.S.P., 93, 242.

Steffen. M. 1985, Astr. Ap. Suppl., 59, 403.

Thévenin, F. and Foy, R. 1986, Astr. Ap., 155, 145.

Trimble, V. 1975, Rev. Mod. Phys., 47, 877.

Unsöld, A. 1955, Physik der Sternatmospharen (Berlin: Springer-Verlag), p.331.

van Genderen, A. M., van Driel, W., and Greidanus, H., 1986, Astr. and Ap., $155,72$.

Wares, G. W., Ross, J. E. and Aller, L.H. 1968, Ap. Space Sci., 2, 344.

Warner, B. 1967, Mem. R.A.S., 70, 165.

Wiese, W. L. and Fuhr, J. R. 1975, J. Phys. Chem. Ref. Data, 4, 263.

Wiese, W. L. and Martin, G. A. 1980, NBS Monog_-No. 68.

Wiese, W. L., Smith, M.W. and Miles, B. M. 1969, NBS Monog., No. 22.

Wolf, B. 1972, Astr. Ap., 20, 275. 1973, Astr. Ap., 28, 335.

Woosley, S. E. 1988, Ap. J., 330, 218.

Younger, S. M., Fuhr, J. R., Martin, G. A. and Wiese, W. L. 1978, J. Phys. Chem. Ref. Data, 7, 495. 


\title{
Abundances of the Heavy Elements in the Magellanic Clouds II. H II Regions and Supernova Remnants
}

\author{
Stephen C. Russell and Michael A. Dopita
}

Mount Stromlo and Siding Spring Observatories, Institute of Advanced Studies, The Australian National University. 


\begin{abstract}
This paper presents the first consistent abundance analysis of both $\mathrm{H}$ II regions and supernova remnants in the Magellanic Clouds, as derived from detailed modeling of spectra extending from $3000-11000 \AA$. The major purpose of the investigation was to find the zero-point error that might exist between the separated abundance scales derived from supergiants (from Russell and Bessell 1989) and $H$ II regions, and thereby determine the first consistent abundance scale from helium to the heaviest elements. An overlap between the two abundance scales was provided by the observation of supernova remnants. Detailed modeling of both the $\mathrm{H} \mathrm{II} \mathrm{regions} \mathrm{and} \mathrm{the} \mathrm{supernova} \mathrm{remnants} \mathrm{was} \mathrm{performed} \mathrm{using} \mathrm{the} \mathrm{same} \mathrm{generalized}$ modeling code MAPPINGS (Binette et al. 1982) thus providing a consistent physical framework for both. For elements heavier than argon, however, a differential analysis was performed between the supernova remnants and the Galactic Herbig-Haro $(\mathrm{H}-\mathrm{H})$ objects. The results showed that there was no measurable zero-point error between the abundance scales of the supergiants and the H II regions.

This result is crucial, and allows us for the first time to connect the two scales and thereby determine important details of the past star formation histories of both the Magellanic Clouds and the Galaxy. The most exciting result is that the elemental abundances relative to iron in the Clouds, match those of the present local interstellar medium more closely than that of the Sun itself, thus suggesting a close similarity between the star formation histories of the Magellanic Clouds and of the local interstellar medium. Conversely, this indicates that the local interstellar medium of the Sun $4.5 \times 10^{9}$ years ago, had a substantially different history of star formation .
\end{abstract}




\section{INTRODUCTION}

Studies of the elemental abundances in the Magellanic Clouds, and their comparison with the solar neighborhood, are of great interest to us as they provide basic information on the rates at which elements are returned to the interstellar medium (ISM) in environments with different amounts of chemical evolution. Each star ejects a particular pattern of elements into the ISM, and it is the integration of the ejecta from all stellar masses that determines the observed pattern. The detailed abundances, therefore, reflect the overall history of star formation in any system.

In Paper I of this series (Russell and Bessell 1989) we determined the detailed abundances for a set of F-supergiants in both Magellanic Clouds. This represented the first such survey, performed in a consistent manner, for both of the Clouds. It is well-known, however, that such observations can not give abundances for the lighter elements: $\mathrm{He}, \mathrm{N}, \mathrm{O}, \mathrm{Ne}, \mathrm{S}, \mathrm{Cl}$ and $\mathrm{Ar}$. These critical elements have traditionally been determined from observations of emission line strengths from $\mathrm{H}$ II regions (see for example Pagel et al., 1978). Analysis of this data in the past has been plagued by imprecise modeling and the lack of observational data on the most abundant ionization species of some of the critical coolants. Furthermore, there has been no attempt to connect the abundances of the heavy elements derived from supergiants with those of the light elements derived from $\mathrm{H}$ II regions.

In an attempt to redress these problems, we measured the optical, and nearinfrared spectra of several bright $\mathrm{H}$ II regions in both Clouds in a consistent manner. The wavelength coverage was therefore extended beyond the range of most previous surveys, and included the [SIII] doublet at $\lambda \lambda 9069,9532 \AA$ (providing a measure of the principal ionization species for S), and the [SII] doublet at $\lambda \lambda 10318$, $10336 \AA$ (providing a potentially valuable measure of the reddening). The objects were then analyzed using the most up-to-date generalized modeling code MAPPINGS, in order to determine their detailed abundances. 
In parallel with our survey of $\mathrm{H} I I$ regions, we measured the optical and nearinfrared spectra of several bright supernova remnants in both Magellanic Clouds in a consistent manner. The advantage of extending the wavelength coverage into the infrared for these objects, is the availability of several forbidden heavy metal lines ([Ca], $[\mathrm{Cr}],[\mathrm{Fe}]$ and $[\mathrm{Nim}]$ ), in addition to the sulphur lines obtained in $\mathrm{H} \Pi$ region spectra. Measuring the heavy metal lines differentially with respect to Galactic $\mathrm{H}-\mathrm{H}$ objects, and modeling the light elements using the same MAPPINGS package as for the $\mathrm{H} \mathrm{II} \mathrm{regions,} \mathrm{allowed} \mathrm{us} \mathrm{to} \mathrm{connect} \mathrm{the} \mathrm{abundances} \mathrm{derived} \mathrm{from} \mathrm{supergiants,}$ with those derived from $\mathrm{H} I I$ regions.

\section{OBSERVATIONS AND REDUCTIONS}

\section{a) Data Acquisition}

All observations were made on the $3.9 \mathrm{~m}$ Anglo-Australian Telescope (AAT) during the 1985 and 1986 observing seasons. For the optical wavelengths (up to $7600 \AA$ ) we used the Image Photon Counting System (IPCS) (Boksenberg 1972; Boksenberg and Burgess 1973) on the Royal Greenwich Observatory (RGO)spectrograph at the $f / 7.9$ Cassegrain focus with the $25 \mathrm{~cm}$ focal-length camera. For those wavelengths beyond the normal optical range, up to an effective limit of $\sim 10,400 \AA$, we used the Faint Object Red Spectrograph (FORS) with a CCD detector. For both instruments the slit length corresponded to 230 arcseconds on the sky. The details of these observations are presented in Table 1 for the $\mathrm{H} \mathrm{II}$ regions, and in Table 2 for the supernova remnants in the Clouds and the Galactic $\mathrm{H}-\mathrm{H}$ objects.

The Magellanic Clouds have been shown to be very well mixed systems (see Pagel et al., 1978), and as such, sampling the abundances at any one position in a Cloud, should reflect the abundances of the Cloud in general. Therefore it was planned that we observe at least three $\mathrm{H} I \mathrm{I}$ regions and supernova remnants (SNRs) 


\section{TABLE 1}

Spectral Log for the HII-Regions

\begin{tabular}{|c|c|c|c|c|c|c|c|c|c|}
\hline \multirow{2}{*}{$\begin{array}{l}\text { Henize } \\
\text { No. }\end{array}$} & \multirow{2}{*}{$\begin{array}{l}\text { DEM } \\
\text { No. }\end{array}$} & \multicolumn{2}{|c|}{ (1950) } & \multirow{2}{*}{ Instr. I } & \multirow{2}{*}{$\begin{array}{l}\text { Dwell } \\
\text { (sec.) }\end{array}$} & \multirow[t]{2}{*}{ Date } & \multirow{2}{*}{\multicolumn{2}{|c|}{$\begin{array}{l}\text { Dispn. Resln. } \\
(\AA / \mathrm{mm}) \quad(\AA)\end{array}$}} & \multirow{2}{*}{$\begin{array}{l}\text { Wavelength } \\
\text { Range }(\AA)\end{array}$} \\
\hline & & $\alpha$ & $\delta$ & & & & & & \\
\hline \multicolumn{10}{|c|}{ SMC } \\
\hline \multirow[t]{3}{*}{$\mathrm{N} 12 \mathrm{~A}$} & 23 & 004439.7 & -732220 & IPCS & 1600 & 19 Sep '86 & 57 & 2 & $3380-5230$ \\
\hline & & & & IPCS & 1600 & & 57 & 2 & $5310-7160$ \\
\hline & & & & FORS & 1000 & & 450 & 23 & $5200-10900$ \\
\hline \multirow[t]{3}{*}{$\mathrm{N} 13 \mathrm{AB}$} & 16 & 004334.7 & -733924 & IPCS & 2000 & 19 Sep '86 & 57 & 2 & $3380-5230$ \\
\hline & & & & IPCS & 1600 & & 57 & 2 & $5310-7160$ \\
\hline & & & & FORS & 1000 & & 450 & 23 & $5200-10900$ \\
\hline \multirow[t]{2}{*}{$\mathrm{N} 25$} & 38 & $\infty 04619.9$ & -733035 & IPCS & 1700 & 4 Oct ' 85 & 143 & 5 & $3200-7600$ \\
\hline & & & & FORS & 660 & & 450 & 23 & $5200-10900$ \\
\hline \multirow[t]{3}{*}{ N64A } & 95 & 005645.7 & -725606 & IPCS & 1600 & 19 Sep '86 & 57 & 2 & $3380-5230$ \\
\hline & & & & IPCS & 2000 & & 57 & 2 & $5310-7160$ \\
\hline & & & & FORS & 1000 & & 450 & 23 & $5200-10900$ \\
\hline \multirow[t]{2}{*}{ N77A } & $117 \mathrm{~b}$ & 010110.6 & -720925 & IPCS & 1500 & 4 Oct ' 85 & 143 & 5 & $3200-7600$ \\
\hline & & & & FORS & 1000 & 3 Oct ' 85 & 450 & 23 & $5200-10900$ \\
\hline \multirow[t]{2}{*}{$\mathrm{N} 84 \mathrm{C}$} & 149 & 011253.8 & -733144 & IPCS & 1500 & 4 Oct ' 85 & 143 & 5 & $3200-7600$ \\
\hline & & & & FORS & 1000 & 3 Oct ' 85 & 450 & 23 & $5200-10900$ \\
\hline \multicolumn{10}{|c|}{ LMC } \\
\hline \multirow[t]{2}{*}{$\mathrm{N} 4 \mathrm{~A}$} & $8 b$ & 045203.9 & -670024 & IPCS & 1100 & 4 Oct ' 85 & 143 & 5 & $3200-7600$ \\
\hline & & & & FORS & 300 & & 450 & 23 & $5200-10900$ \\
\hline \multirow[t]{3}{*}{ N127A } & 149 & 052157.9 & -694314 & IPCS & 1000 & 19 Sep '86 & 57 & 2 & $3380-5230$ \\
\hline & & & & IPCS & 1000 & & 57 & 2 & $5310-7160$ \\
\hline & & & & FORS & 500 & & 450 & 23 & $5200-10900$ \\
\hline \multirow[t]{3}{*}{ N138A } & 180 & 052518.1 & -683055 & IPCS & 1600 & 19 Sep '86 & 57 & 2 & $3380-5230$ \\
\hline & & & & IPCS & 1700 & & 57 & 2 & $5310-7160$ \\
\hline & & & & FORS & 500 & & 450 & 23 & $5200-10900$ \\
\hline \multirow[t]{2}{*}{ N179A } & 320 & 054826.3 & -695351 & IPCS & 1000 & 4 Oct ' 85 & 143 & 5 & $3200-7600$ \\
\hline & & & & FORS & 1000 & 3 Oct ' 85 & 450 & 23 & $5200-10900$ \\
\hline
\end{tabular}




\section{TABLE 2}

Spectral Log for the Supernova Remnants

\begin{tabular}{|c|c|c|c|c|c|c|c|c|}
\hline \multirow{2}{*}{$\begin{array}{l}\text { Henize } \\
\text { Number }\end{array}$} & \multirow{2}{*}{$\begin{array}{l}\text { Other } \\
\text { Number }\end{array}$} & \multicolumn{2}{|c|}{ (1950) } & \multirow{2}{*}{$\begin{array}{l}\text { Instr- } \\
\text { ument }\end{array}$} & \multirow{2}{*}{$\begin{array}{l}\text { Dwell } \\
\text { (sec.) }\end{array}$} & \multirow{2}{*}{ Date $I$} & \multirow{2}{*}{\multicolumn{2}{|c|}{$\begin{array}{l}\text { Dispn. Wavelength } \\
(\AA / \mathrm{mm}) \text { Range }(\AA)\end{array}$}} \\
\hline & & $\alpha$ & $\delta$ & & & & & \\
\hline & & & & $\mathrm{MC}$ & & & & \\
\hline \multirow[t]{2}{*}{ N19 } & SNR 0045-734 & 004524.1 & -732432 & 2 IPCS & 1700 & 04 Oct '8 & $85 \quad 143$ & $3200-7600$ \\
\hline & & & & FORS & 3000 & & 660 & $5200-10900$ \\
\hline \multirow[t]{4}{*}{ - } & SNR 0104-723 & 010435.2 & -722157 & 7 IPCS & 3000 & 26 Dec ' 8 & '86 57 & $3200-5590$ \\
\hline & & & & IPCS & 2102 & & 57 & 4745.7200 \\
\hline & & & & FORS & S 1000 & 03 Oct '8 & '85 660 & $5200-10900$ \\
\hline & & & & FORS & S 550 & $26 \operatorname{Dec} ' \varepsilon$ & '86 660 & $5200-10900$ \\
\hline
\end{tabular}

\section{LMC}

\begin{tabular}{|c|c|c|c|c|c|c|c|c|}
\hline \multirow[t]{2}{*}{ N49 } & SNR 0525-66.1 & 052600.5 & -660742 & IPCS & 2000 & 04 Oct ' 85 & 143 & $3200-7600$ \\
\hline & & & & FORS & 2000 & 03 Oct ' 85 & 660 & $5200-10900$ \\
\hline \multirow[t]{2}{*}{ N63A } & SNR 0535-66.0 & 053537.8 & -660354 & IPCS & 1000 & 04 Oct ' 85 & 143 & $3200-7600$ \\
\hline & & & & FORS & 2000 & 03 Oct ' 85 & 660 & $5200-10900$ \\
\hline \multirow[t]{5}{*}{$\mathrm{N} 103 \mathrm{~b}$} & SNR 0509-68.7 & 050912.3 & -684726 & IPCS & 1000 & 26 Dec '86 & 57 & $3200-5590$ \\
\hline & & & & IPCS & 500 & & 57 & $4745-7200$ \\
\hline & & & & FORS & 2000 & 03 Oct ' 85 & 660 & $5200-10900$ \\
\hline & & & & FORS & 1000 & 26 Dec '86 & 660 & $5200-10900$ \\
\hline & \multicolumn{8}{|c|}{ Galactic } \\
\hline \multirow[t]{3}{*}{ - } & $\mathrm{HH} 1$ & 053355.1 & -064701 & IPCS & 400 & 04 Oct '85 & 143 & $3200-7600$ \\
\hline & & & & FORS & 500 & 03 Oct '85 & 660 & $5200-10900$ \\
\hline & & & & FORS & 500 & $26 \mathrm{Dec} ' 86$ & 660 & $5200-10900$ \\
\hline \multirow[t]{2}{*}{ - } & $\mathrm{HH} 32 \mathrm{~A}, \mathrm{~B}$ & 191808.1 & +105621 & IPCS & 1500 & 04 Oct ' 85 & 143 & $3200-7600$ \\
\hline & & & & FORS & 2000 & & 660 & $5200-10900$ \\
\hline \multirow[t]{2}{*}{ - } & HH56 & 162853.8 & -444837 & IPCS & 3000 & 04 Oct '85 & 143 & $3200-7600$ \\
\hline & & & & FORS & 2000 & 03 Oct '85 & 660 & $5200-10900$ \\
\hline \multirow[t]{2}{*}{-} & HH101N,S & 185812.4 & -370718 & IPCS & 1500 & 04 Oct ' 85 & 143 & $3200-7600$ \\
\hline & & & & FORS & 1000 & & 660 & $5200-10900$ \\
\hline
\end{tabular}


in each Cloud. As can be seen from Table 1, we were successful in this respect in the case of the $\mathrm{H} \mathrm{II}$ regions (4 in the LMC and 6 in the SMC). Unfortunately, we only managed to make complete observations of 3 SNRs in the LMC and 2 in the SMC due to cloudy observing conditions (see Table 2). However, for one of the SNRs in the SMC (SNR 0104-723) no previous spectra have been reported in the literature (we are therefore doubling the number of SNRs measured in that galaxy). In addition, we did obtain a FORS spectrum of another SNR in the SMC (SNR 0050-728). This was, unfortunately, of poor quality, and we were unable to obtain any observations in the blue part of the spectrum.

The two main criteria we adopted for our choice of $\mathrm{H}$ II regions suitable for observation in the Clouds were as follows:

1) they should be as bright as possible, allowing us to attain high signal-tonoise data in reasonable integration times;

2) they should be compact and as nearly spherical as possible to ensure they closely match the physical conditions assumed in our generalized modeling code, MAPPINGS (see § IV for a description).

The fact that they are bright means that many have been previously investigated in the literature, and although this was not taken as a criterion, the literature often provided very useful comparisons.

In the case of the SNRs, the main criteria were:

1) They should be as bright as possible;

2) they should be 'mature' SNRs.

If a SNR is too young, then the emission spectrum is dominated by emission from the gas ejected by the supernova itself (see Dopita, Tuohy, and Mathewson 1981; Dopita and Tuohy, 1984).

In order for us to reliably determine the abundances of the heavier elements ( $\mathrm{Ca}, \mathrm{Cr}, \mathrm{Fe}$ and $\mathrm{Ni}$ in particular), we needed reference objects having essentially known abundances for comparison. Thus we chose to observe a selection of the 
brighter $\mathrm{H}-\mathrm{H}$ objects available to us during our observing runs. These objects are now recognized as being small knots of shock excited gas in the local interstellar medium (as first suggested by Schwartz, 1975), and since their shock velocities are, in some cases, quite high, their spectral characteristics overlap with the SNRs. In choosing our $\mathrm{H}-\mathrm{H}$ objects (see Table 2), we ensured that their spread of excitation conditions were similar to those we expected for the SNRs.

The generalized modeling code MAPPINGS (see §.III) was designed to work on the integrated spectra from emission objects, since the vast majority of such objects available for study in the universe are not spatially resolved. In order to measure the integrated spectra from most of our spatially resolved objects, we scanned the telescope over the whole region. This has the advantage of making our observations independent of position of the slit on the object (a problem that has dogged previous comparisons between one investigator and another), and allows us to judge the effect of observing the integrated spectra for objects whose detailed structure is well determined. For the two faint SNRs in the SMC, however, the slit was held stationary along a single bright filament (two separate filaments in the case of N19) in order to achieve the highest possible signal-to-noise.

The observations were reduced using the Mount Stromlo Observatory (MSO) computing package PANDORA. After bias subtraction (for observations made with FORS), and flatfielding, the spatial increments containing the 'sky background' were averaged, then subtracted from the frame. Quite often the 'sky background' itself was polluted by faint, underlying emission nebulae, however, such extraneous emission must also underlie the objects themselves, and it is therefore quite proper to remove this along with the sky.

Wavelength calibration was carried out using $\mathrm{Cu} / \mathrm{Ar}$ comparison arcs, and after mild filtering, those object rows unaffected by stellar continua, were extracted. The relative flux calibration for the IPCS observations was performed using the flux standards: LDS749B, L745-46 A, VMA2 and L780-2 from Oke (1974). The FORS 
data was first divided through by a smooth spectrum standard (observed at several air-masses whenever possible) to remove the atmospheric absorption features, before flux calibrating using the standards: HR1790 and HR1544 (from Taylor 1984) and HD19445 (from Oke and Gunn 1983).

The IPCS remains linear until the count rate exceeds $\sim 0.8 \mathrm{~Hz}$ (for the case of emission lines), however, to obtain good signal-to-noise in the weakest lines one would like to count as fast as possible, thus incurring saturation in the stronger lines. In order to overcome this problem we used two strategies. Firstly, in the case of the September 1986 observations, we ensured that we had a short exposure of each object with enough filtering to reduce the count rate for the strongest lines below the required limit. Thus, on this occasion, we could be confident in setting the dereddened $\mathrm{H} \alpha$ intensity to the theoretical value (from Brocklehurst, 1971) of 285.9 relative to $H \beta=100$. All remaining saturation in our data was taken out by forcing the ratio of the [OII] doublet $I(\lambda 5007) / I(\lambda 4959)$ to be equal to its theoretical value of 2.96 in each row of the two-dimensional spectra. This gave a wide range of intensities over which the effects of saturation could be calibrated out with a smooth curve, recalculated for each observing run. Although this was satisfactory for most lines, $\mathrm{H} \alpha$ was sometimes too strong to be covered by the intensity range of the [OII] lines. It was mainly because of this fact that the $\mathrm{H}_{\alpha} / \mathrm{H} \beta$ ratio was given a low weighting in our computation of the reddenings (see §IIb).

For the spectra obtained at a resolution of $5 \AA$, the [SII $\lambda \lambda \lambda 6716,6731$ doublet was deblended using the Gaussian fitting routines of the SPECTRE code (Pelat, Alloin and Fosbury, 1981). Several other lines were also deblended using this code, then all of the measured line intensities were placed on a scale where $I(\mathrm{H} \beta)=$ 100, and are tabulated in Appendix A (Tables A1 and A2) for the sake of completeness. The first three columns give, respectively, the ionic species and the corresponding laboratory wavelengths and multiplet numbers for the transitions thought to be responsible for the observed emission lines. The line list was selected 
by careful comparisons between our own spectra and those published in the literature (see Table 3), backed up by the Multiplet Table of Charlotte Moore (1945). On the occasions when the choice of a possible transition was very uncertain, we followed our listed ionic species with a question-mark.

Beyond $\sim 9600 \AA$, the color filter we were using (GG495) began to transmit second order blue light. This resulted in a strong detection of the second order $\mathrm{H} \beta$ and [OIII] $\lambda \lambda 4959,5007$ lines (the latter of which masked the carbon lines near 9830 $\AA$ in the SNRs), and an overestimate of the standard stellar continuum flux. As a consequence of this, the lines beyond $9600 \AA$ were artificially depressed. A correction factor was derived from an extrapolation of the observed flux of the standard stars, assuming them to be "well behaved" beyond $9600 \AA$, and applied to all our tabulated line-strengths.

\section{b) Reddening}

The lines were corrected for interstellar reddening, assuming the Whitford (1958) reddening law, using the formula:

$$
I_{C}=I_{O} \times 10 c f(\lambda) \text {, }
$$

equation 1.

where $I_{C}$ and $I_{O}$ are the corrected and observed intensities respectively, and $f(\lambda)$ is the reddening function as tabulated by Kaler (1976). The logarithmic reddening constant $c\left(\mathrm{H}_{\beta}\right)$ is defined by:

$$
c\left(\mathrm{H}_{\beta}\right)=\left[\log F_{c}\left(\mathrm{H}_{\beta}\right)-\log F_{o}\left(\mathrm{H}_{\beta}\right)\right],
$$

equation 2.

where $F_{C}(\mathrm{H} \beta)$ and $F_{o}(\mathrm{H} \beta)$ are the correct and the observed $\mathrm{H} \beta$ fluxes respectively.

We derived the reddening constant $c\left(\mathrm{H}_{\beta}\right)$ from two different methods whenever possible. The first was from a comparison of the observed Balmer decrements, with the theoretical decrements from Brocklehurst (1971), assuming case B (as formulated by Baker and Menzel 1938) for a temperature of $10000 \mathrm{~K}$ and an electron density of $100 \mathrm{~cm}^{-3}$. Of the various Balmer ratios that could be measured with any confidence, we gave a weight of 2 to $H(\delta) / H(\beta)$ and $H(\gamma) / H(\beta)$, 
TABLE 3

References for the HII Regions

\begin{tabular}{|c|c|c|c|c|c|}
\hline \multicolumn{6}{|c|}{ HII Regions } \\
\hline & \multicolumn{2}{|c|}{ SMC } & \multicolumn{2}{|r|}{ LMC } & \multirow[b]{2}{*}{$\lambda$-Range $(\AA)$} \\
\hline & $\mathrm{N} 12 \mathrm{~A} \quad \mathrm{~N} 1$ & AB $\quad \mathrm{N} 25$ & N64A & N4A N127A & \\
\hline Dennefeld and Stasinska, 1983 & ... & $\mathrm{x}$ & $\ldots$ & $\mathrm{X}$ & $6584-10830$ \\
\hline Dickel et al., 1964 & $\mathrm{X}$ & $\ldots$ & $\ldots$ & $\ldots$ & $3727-6723$ \\
\hline Dufour and Harlow, 1977 & $\mathrm{X}$ & $\mathrm{X}$ & $\ldots$ & $\ldots$ & $3727-6723$ \\
\hline Pagel et al., 1978 & $\ldots$ & $\mathrm{X}$ & $\mathrm{X}$ & $\ldots$ & $3727-7324$ \\
\hline Peibert and Torres-Peimbert, 1974 & $\ldots$ & $\ldots$ & $\ldots$ & $\ldots$ & $3727-7330$ \\
\hline Webster, 1976 & \multicolumn{2}{|c|}{$\cdots$} & $\mathrm{X}$ & $\cdots$ & $3727-5007$ \\
\hline \multicolumn{6}{|c|}{ SNRs } \\
\hline & SMC & \multicolumn{3}{|c|}{ LMC } & \multirow[b]{2}{*}{$\lambda$-Range $(\AA)$} \\
\hline & N19 & $\mathrm{N} 49$ & N63A & $\mathrm{N} 103 \mathrm{~b}$ & \\
\hline Danziger and Leibowitz, 1985 & $\ldots$ & ... & $\mathbf{X}$ & $\mathbf{X}$ & $3727-7295$ \\
\hline Dennefeld, 1986 & $\ldots$ & $\mathbf{X}$ & $\mathbf{X}$ & ... & $6300-10500^{\mathrm{a}}$ \\
\hline Dopita, Mathewson and Ford, 1977 & $\mathrm{x}$ & $\mathrm{X}$ & $\mathrm{X}$ & $\mathbf{X}$ & $6300-6731^{b}$ \\
\hline \multirow[t]{3}{*}{ Osterbrock and Dufour, 1973} & $\cdots$ & $\mathrm{X}$ & $\cdots$ & $\cdots$ & $3727-6731$ \\
\hline & \multicolumn{2}{|c|}{ H-H Objects } & & & \\
\hline & $\mathrm{HHI}$ & $\mathrm{HH} 32 \mathrm{AB}$ & HH56 & 5 HH101N,S & $\lambda$-Range $(\AA)$ \\
\hline Böhm, Perry and Schwartz, 1973 & $\mathrm{x}$ & ... & ... & ... & $3726-6830$ \\
\hline Bobhm, Siegmund and Schwartz, 1976 & $\mathrm{x}$ & ... & ... & ... & $3727-10830$ \\
\hline Brugel, Böhm and Mannery, 1981 & $\mathbf{x}$ & $\mathbf{X}$ & $\ldots$ & $\ldots$ & $3727-10830$ \\
\hline Dopita, 1978 & $\mathrm{x}$ & $\mathrm{X}$ & $\ldots$ & $\mathrm{x}$ & $4068-7376$ \\
\hline Dopita, Binette and Schwartz, 1982 & $\mathrm{X}$ & $\ldots$ & $\ldots$ & $\ldots$ & $3727-7376$ \\
\hline Hartmann and Raymond, 1984 & $\mathrm{X}$ & $\ldots$ & $\ldots$ & $\ldots$ & $3889-6724^{C}$ \\
\hline Schwartz, 1976 & $\mathrm{X}$ & $\ldots$ & $\ldots$ & $\ldots$ & $3727-7155$ \\
\hline Schwartz and Dopita, 1980 & $\ldots$ & $\ldots$ & $\mathbf{X}$ & $\ldots$ & $3727-7330$ \\
\hline Solf, Bohm and Raga, 1988 & $\mathbf{X}$ & $\ldots$ & $\ldots$ & $\ldots$ & $3727-10830$ \\
\hline Strom, Grasdalen and Strom, 1974 & $\ldots$ & $\ldots$ & $\ldots$ & $\mathrm{X}$ & $4861-6731$ \\
\hline
\end{tabular}

a An additional spectrum was obtained for N49 in the range 3727-7453 $\AA$.

b The spectral range for $\mathrm{N} 19$ was 4861-6731 $\AA$.

c The line intensities used for comparison with our own results were an average from all 5 positions measured. 
a weight of 1 to $H(9) / H(\beta)$ (used for the September 1986 observations only), and 1 to $H(\alpha) / H(\beta)$ (for the October 1985 observations). The latter ratio was felt to be of lesser accuracy due to the effects of possible collisional excitation, the increased risk of poor background subtraction and inadequate saturation correction, and imprecisely determined contamination from $[\mathrm{NI}] \lambda 6548 \AA$.

The second method involved a comparison of the measured ratio:

$$
R=\frac{([\operatorname{SII}] \lambda \lambda 6717,6731)}{([\operatorname{SI}] \lambda \lambda 4068,4076)} \cdot \frac{([\mathrm{OI}] \lambda \lambda 7318,7328)}{([\mathrm{OI}] \lambda \lambda 3727,3729)}
$$

equation 3.

with the theoretical one, as suggested by Allen (1979) and further discussed by Dopita, Binette and Schwartz (1982). As in the latter paper, we deblended ([OI] $\lambda \lambda 33727,3729 \AA$ ) from [Cam] $\lambda 7324 \AA$ (where it was observed), by assuming [CaII] $\lambda 7324 \AA$ has an intensity of two-thirds of the [Cam] $\lambda 7291 \AA$ line. The models presented in their paper however, were only for solar abundance shocks, but further modeling showed that the application of these models to Magellanic Cloud SNRs would involve a negligible increase in the errors in the derived reddening constant. We could therefore use the smooth fit to the models given in figure 2 of Dopita, Binette and Schwartz (1982) for the Cloud SNRs and the Galactic $\mathrm{H}-\mathrm{H}$ objects. The reddening constant was then simply derived from the formula:

$$
\mathrm{R}_{\mathrm{Int}}=\mathrm{R}_{\mathrm{Obs}} \times 10^{\mathrm{cF}}(\lambda), \quad \text { equation } 4 .
$$

where $R_{I n t}$ and $R_{O b s}$ are the intrinsic and the observed ratios respectively, and the combined reddening function:

$$
F(\lambda)=f \lambda 6724-f \lambda 4072+f \lambda 7323-f \lambda 3728=-1.288 . \quad \text { equation } 5 .
$$

There was also every reason to expect that the ratio $R$, would be of use in determining the reddening for the $\mathrm{H} I I$ regions. We performed extensive modeling of these objects over the range of parameters:

$$
\begin{gathered}
37700 \mathrm{~K}<T_{\text {ion }}<43800 \mathrm{~K}, \\
0.08 \times 10^{8} \mathrm{~cm}^{-1}<\bar{Q}<1.8 \times 10^{8} \mathrm{~cm}^{-1},
\end{gathered}
$$




$$
\begin{gathered}
0.690<\left[\mathrm{SI}_{\mathrm{R}}<0.879,\right. \\
1.0 \times 10^{-4}<\mathrm{O} / \mathrm{H}<4.0 \times 10^{-4},
\end{gathered}
$$

where $T_{\text {ion }}$ and $\bar{Q}$ are the effective ionization temperature of the central source (assumed to be that of a single, centrally located star) and the mean ionization parameter (see § III) respectively, and $[S]_{R}$ is the density sensitive ratio ([SI] $\lambda 6731) /([S I]] \lambda 6716)$. A sample of these models is shown in Figure 1, from which we found that the ratio $R$, was mainly dependant upon the oxygen abundance, though the effect was not large. We therefore chose to set $R_{\text {int }}$ equal to 0.41 for the SMC and 0.35 for the LMC. Even if we were simply to ignore the effects of metallicity on $R_{\text {int }}$, and set it equal to 0.37 , the maximum error we would expect to incur in the derivation of the reddening constant $c(\mathrm{H} \beta)$, would be less than 0.05 dex.

In addition to the above two methods for determining the reddening constant, we also attempted to make a more direct measure of the constant by following the suggestion of Miller (1968). This method makes use of the fact that [SI] $\lambda 10318$ and [SII] $\lambda 4069$ are derived from the same upper level, as are [SII] $\lambda 10336$ and [SI] $\lambda 4076$. Owing to our low resolution in the infrared, our procedure involved measuring the combined strength of the violet lines [SII] $\lambda \lambda 4069,4076$ and the infrared lines [SI] $\lambda \lambda \lambda 10318,10336$. From our models we determined that the ratio $I(\lambda 4069) / I(\lambda 4076)$ was always very close to 3.04 over the whole range of physical conditions of interest to us here. This, it should be noted, is substantially higher than the value of 1.17 derived by Böhm, Schwartz, and Siegmund (1974), and assumed in their subsequent papers. The difference is probably due to our allowance for collisional redistribution between doublet levels and our use of more accurate atomic data. Using the transition probabilities of Mendoza and Zeippen (1982), we determined the intrinsic ratio of the combined infrared lines $I(I R)$, to the combined violet lines $I(V)$, to be:

$$
I(I R) / I(V)=0.32 .
$$


Figure 1 The dependence of the reddening sensitive ratio $\mathrm{R}=([\mathrm{SI}] \lambda \lambda 6717,31) .([\mathrm{OI}] \lambda \lambda 7318,28) /([\mathrm{SI}] \lambda \lambda 4068,76) .([\mathrm{OI}] \lambda \lambda 3727,29)$ on the density sensitive ratio [SI] $\lambda 6731 /[\mathrm{SI}] \lambda 6717$ for a selection of our photoionization models. The open circles represent models with $1 \times 10^{-4}<\mathrm{N}(\mathrm{O} / \mathrm{H})<2 \times 10^{-4}$, the filled circles represent $2 \times 10^{-4}<\mathrm{N}(\mathrm{O} / \mathrm{H})<3 \times 10^{-4}$ and the open squares $3 \times 10^{-4}<\mathrm{N}(\mathrm{O} / \mathrm{H})<4 \times 10^{-4}$. 


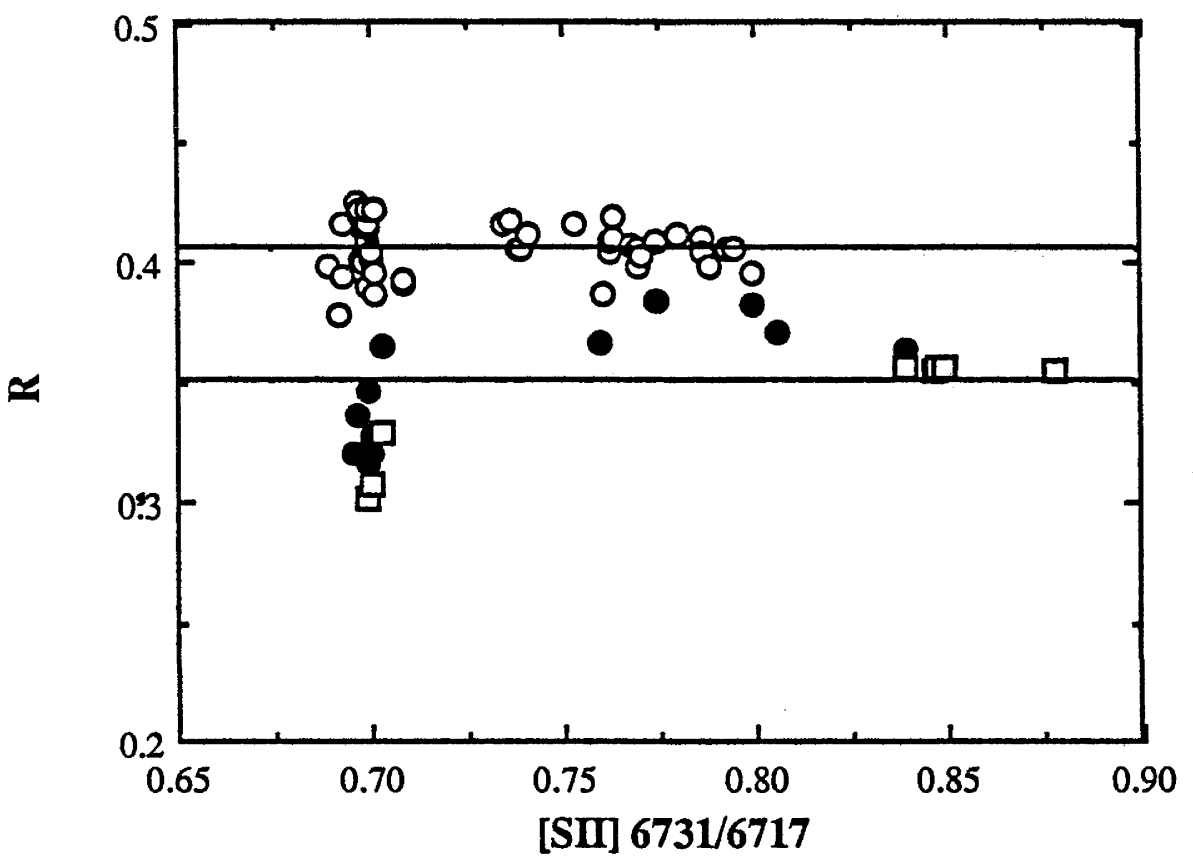


We were, however, tentative about using the method in this work due to the large corrections that were applied to take out the second-order blue leak around $[\operatorname{SII}] \lambda 10330$, the weakness of these lines in many of the objects, and the very low detector sensitivity in this part of the spectrum.

For the $\mathrm{H} I I$ regions and N19, which have particularly weak lines at $10330 \AA$, we found the average difference between the reddening constants derived using Miller's (1968) method and those derived using the mean of the first two methods mentioned in this section was:

$$
c(\mathrm{H} \beta)_{\text {Miller }}-c(\mathrm{H} \beta)_{\text {Adopted }}=+0.25 \pm 0.55 \mathrm{dex}
$$

However when considering the stronger lined SNRs in the LMC and the Galactic $\mathrm{H}-\mathrm{H}$ objects the difference was satisfyingly small:

$$
c\left(\mathrm{H}_{\beta}\right)_{\text {Miller }}-c(\mathrm{H} \beta)_{\text {Adopted }}=0.00 \pm 0.09 \mathrm{dex}
$$

Nevertheless, for the sake of consistency, we adopted reddening constants for all our objects based on the first two methods only.

\section{c) Line Strengths}

The dereddened line strengths are tabulated in Tables 4 and 5, the formats of which are exactly the same as those for Tables A1 and A2 in Appendix A discussed previously. The finally adopted values for the reddening constant $c(\mathrm{H} \beta)$ are included at the bottom of the tables. These were compared with the reddening constants derived for the same objects from the literature (Brugel, Böhm, and Mannery 1981; Dennefeld 1986; Dopita, Mathewson, and Ford 1977; Danziger, and Leibowitz 1985; Schwartz, and Dopita 1980; Böhm, Perry, and Schwartz 1982; Dopita, Binette, and Schwartz 1982; Peimbert and Torres-Peimbert 1974; 


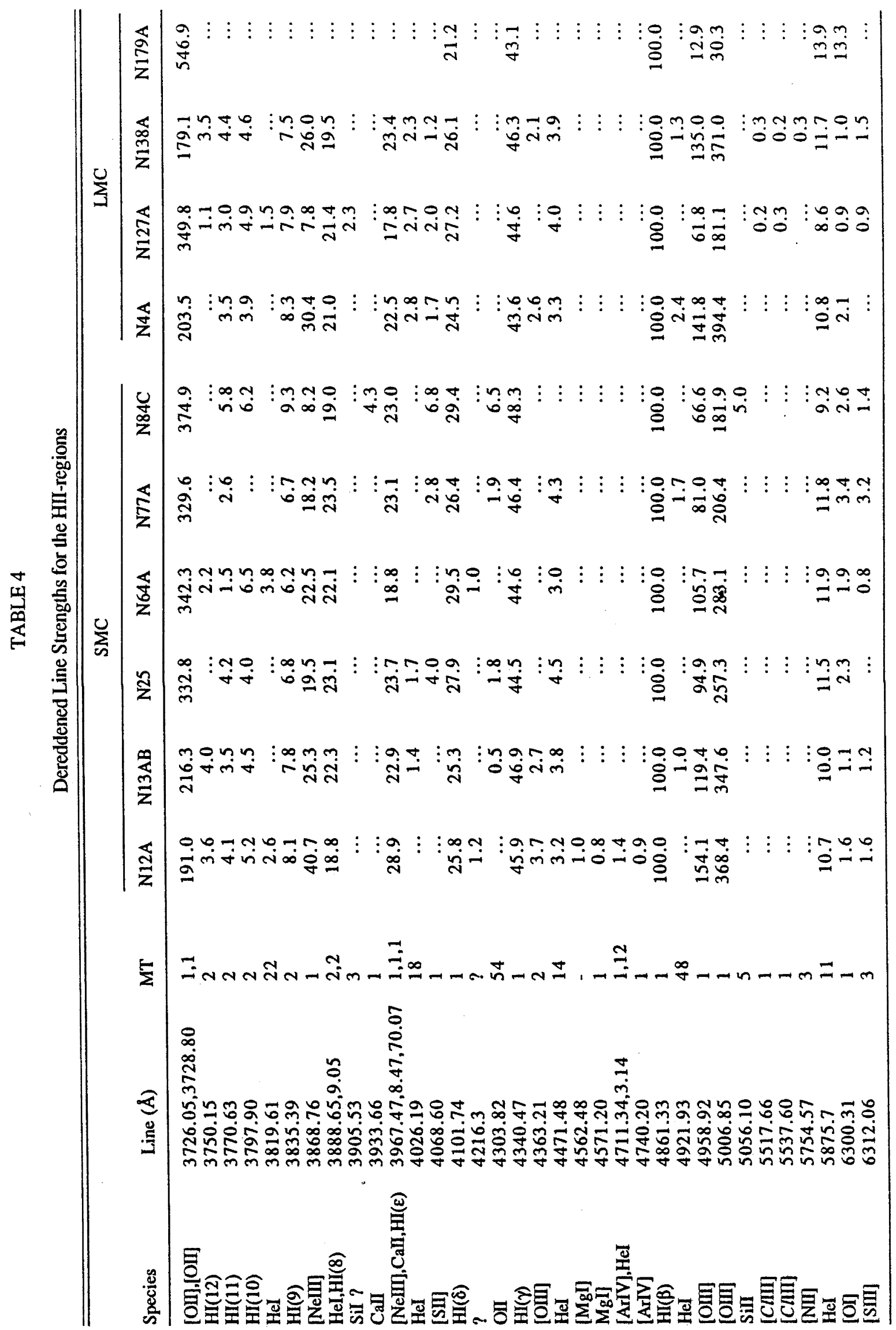




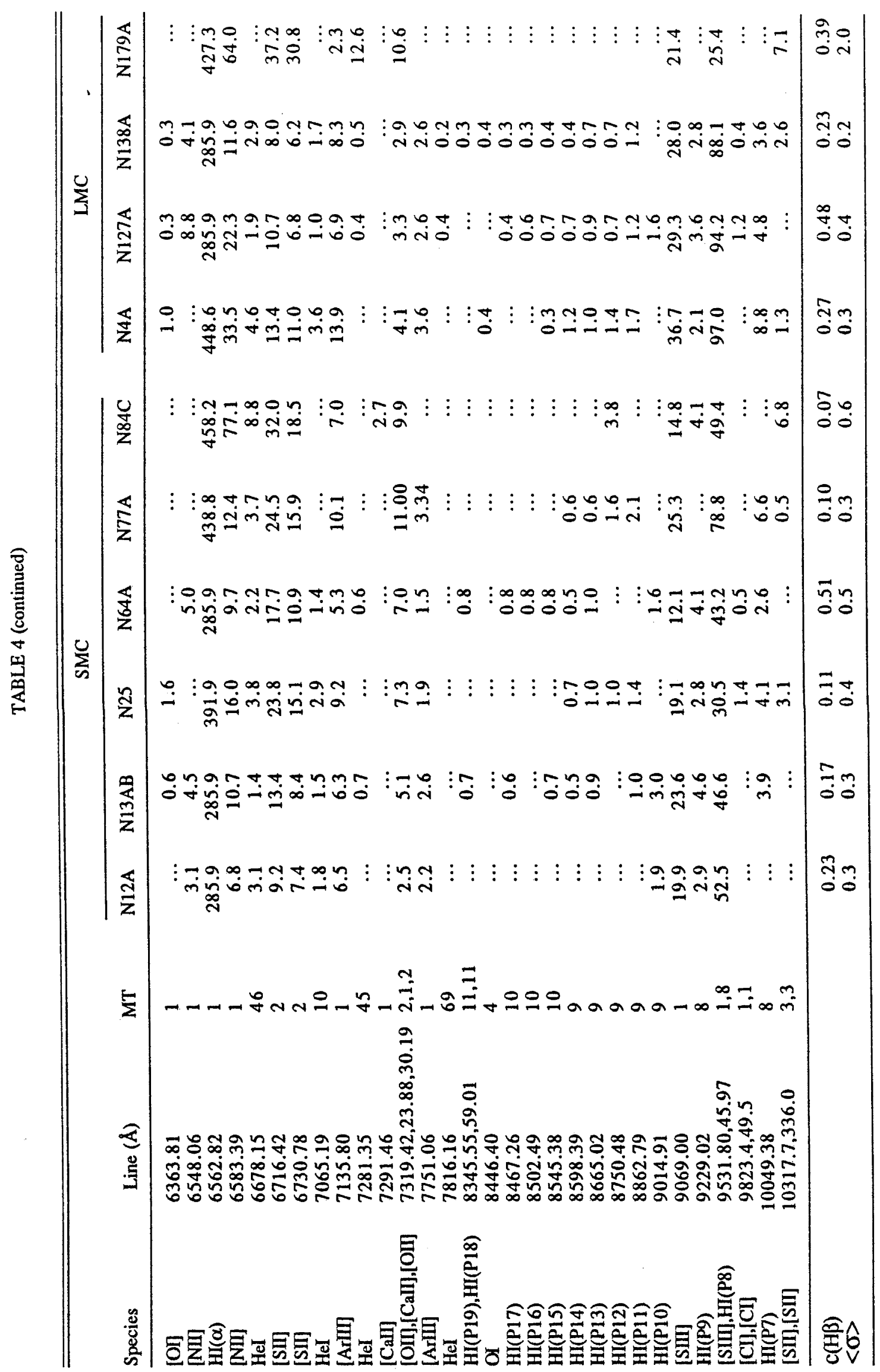




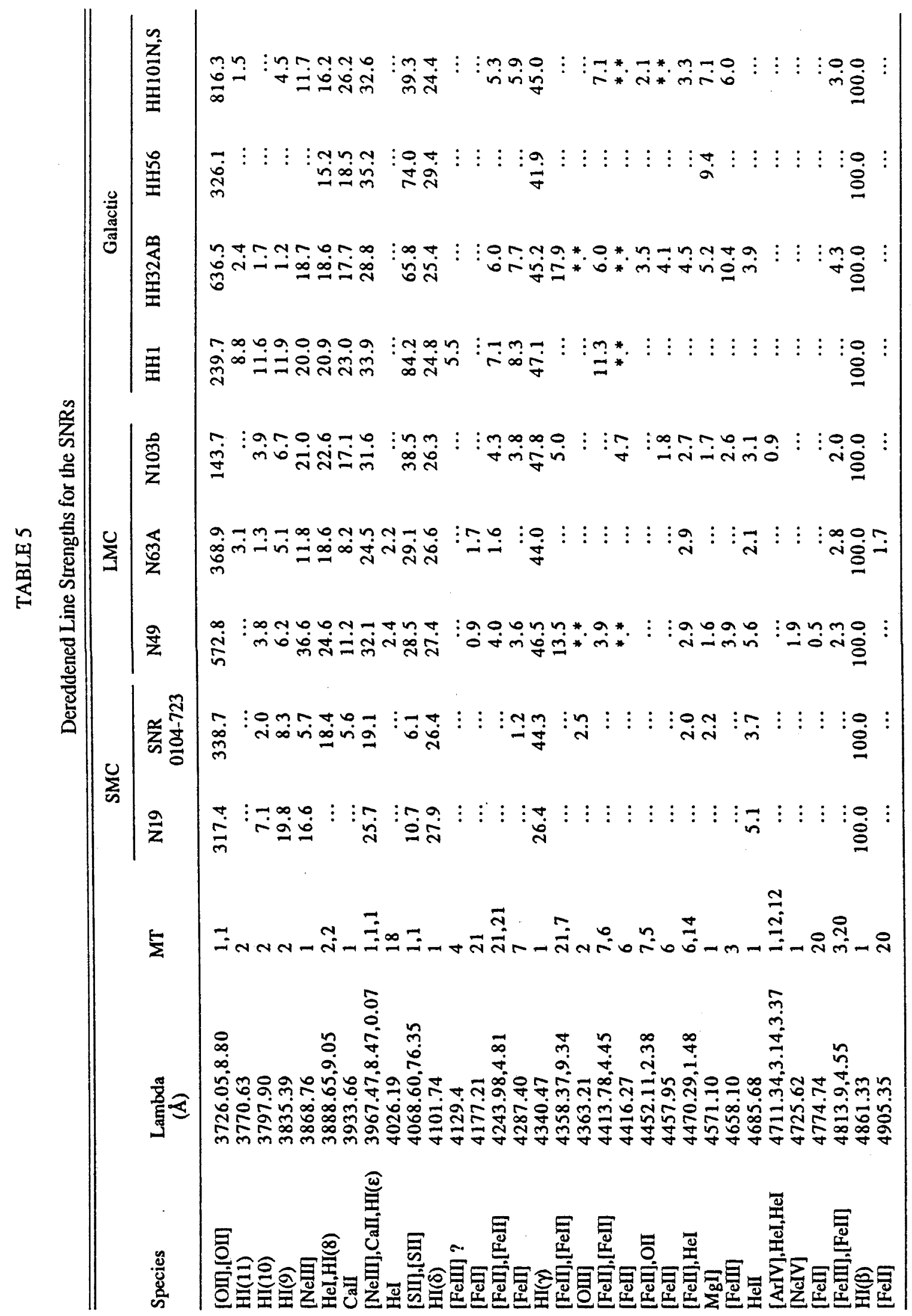




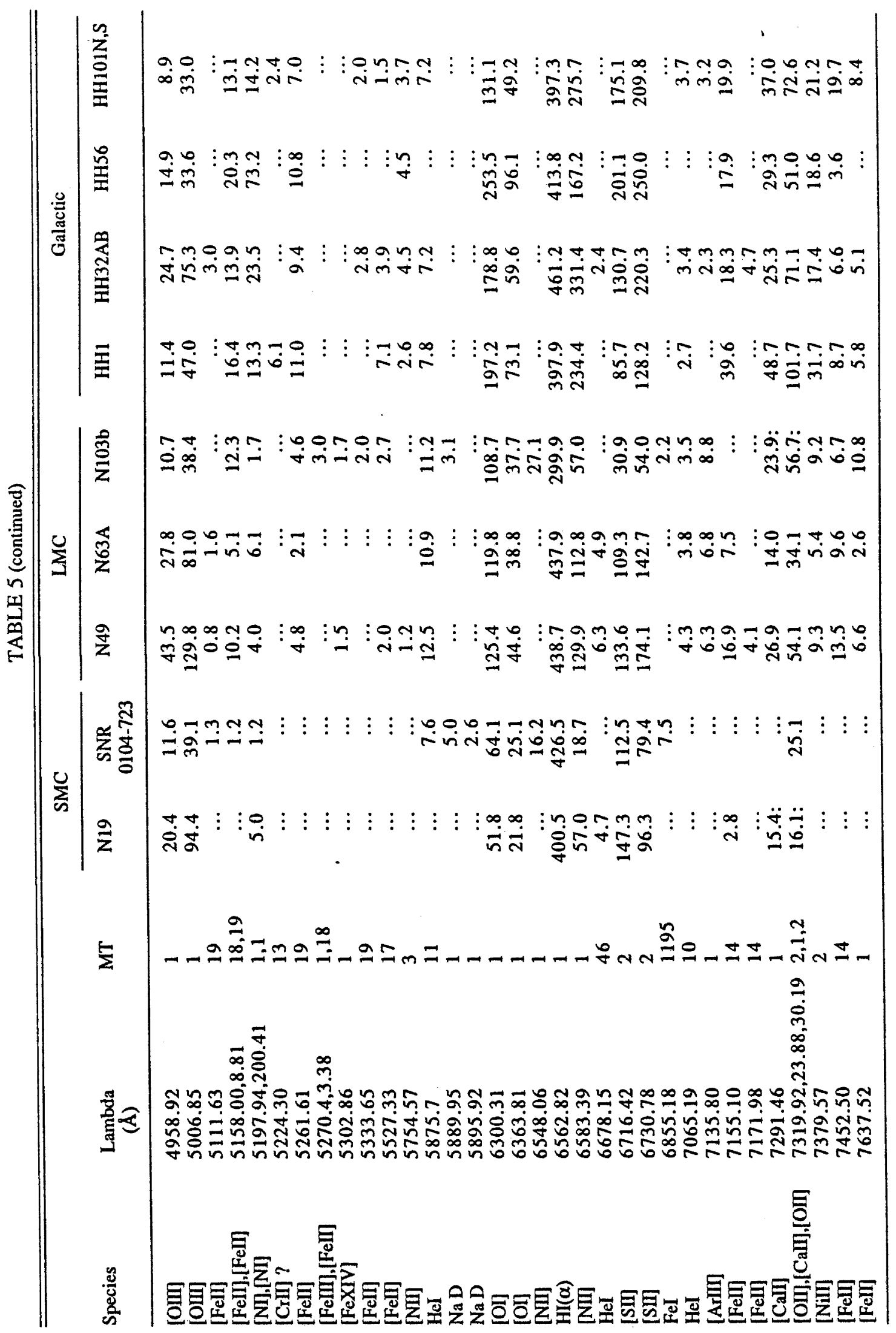




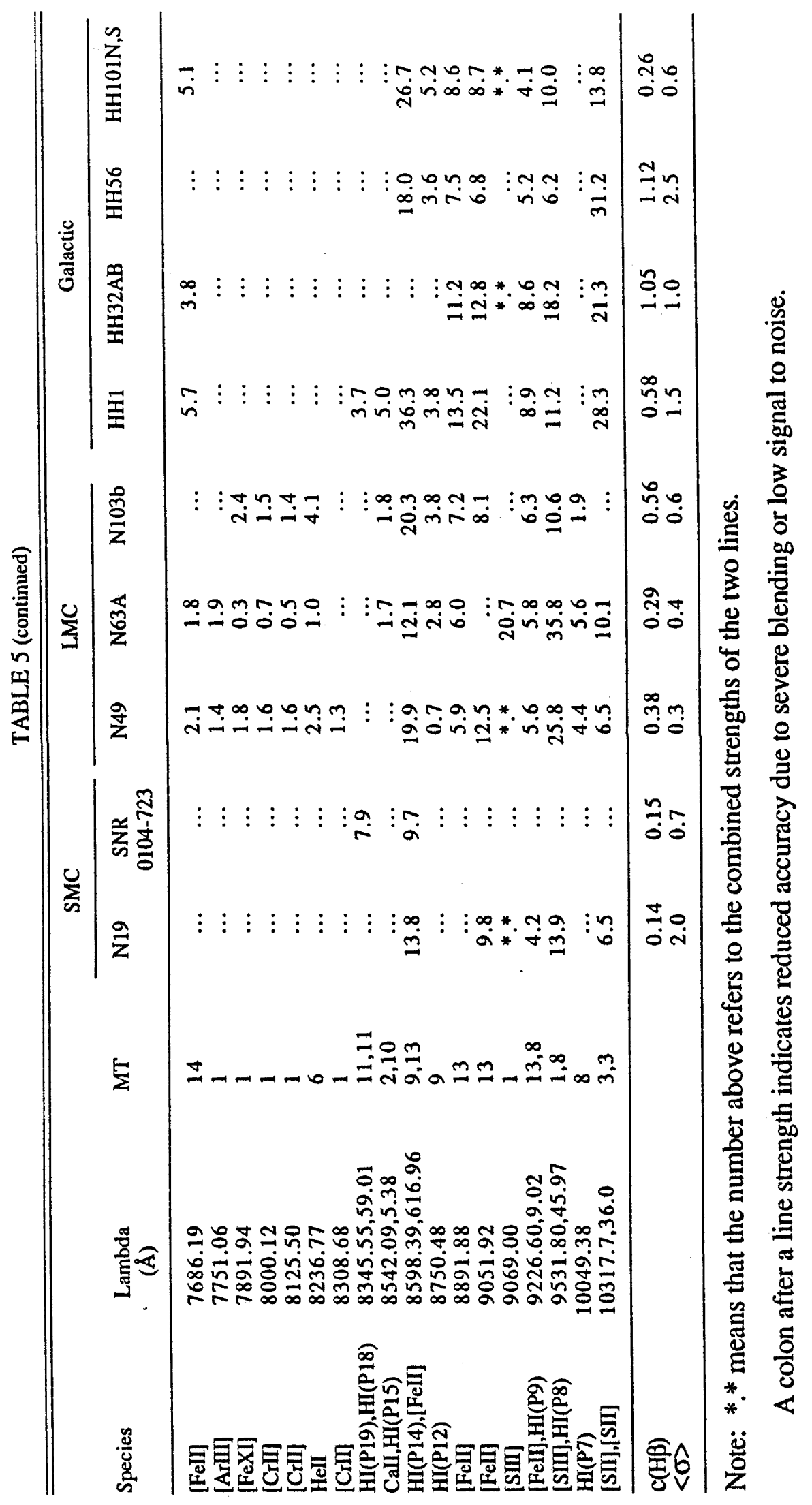


Dufour and Harlow 1977; Dickel, Aller, and Faulkner 1964) with the following result:

$$
c(\mathrm{H} \beta)_{\text {This work }} c(\mathrm{H} \beta)_{\text {Literature }}=-0.01 \pm 0.1 \mathrm{dex} .
$$

The mean standard deviation of the continuum from its mean $\langle\sigma\rangle$, is also included at the bottom of Tables 4 and 5. The main use of $\langle\sigma\rangle$ is to provide a convenient measure of the quality of one spectrum as compared to another. This is further facilitated by reference to the example spectra displayed in Figures 2 to 6 , and allows one to judge for oneself the significance to be placed on the strength, or even the detection, of a weak line. Figures $2 a$ to $2 c$ show example spectra of a SNR, H-H object and an $\mathrm{H}$ II region respectively, in the spectral region from 7000 $\AA$ to $10500 \AA$. In the first two spectra, the full resolution of the IPCS has been maintained over the region from $7000-7500 \AA$, but for the $H$ II region we have retained the FORS resolution throughout. Clearly, the spectrum of HH1 has the same general form as $\mathrm{N} 49$ in the near infrared (further justifying our use of $\mathrm{H}-\mathrm{H}$ objects for comparison spectra), and is quite distinct from that of an $\mathrm{H} \mathrm{II}$ region. Similarly, Figures $3 a$ to $3 c$ display example spectra for SNRs and $\mathrm{H}$ II regions in the optical region of the spectrum. Figure $3 a$ shows the first detailed spectrum of the supernova remnant SNR 0104-723, the only SNR besides N19 in the SMC to have its spectrum measured. Figure $3 b$ shows N84C in the SMC, a rather 'typical' H II region spectrum, certainly so when compared with that of N179A shown in Figure $3 c$.

This is the first reported spectrum of N179A, and we see immediately that it is not clearly categorized as an H II region, unless it has an unusually low $\bar{Q}$, and $\mathrm{T}_{\text {ion. }}$. As can be seen from Tables 4 and 5 , both the [OI] $\lambda 6300$ line and the [SII] $\lambda \lambda 6716,6731$ lines are stronger than for any other $H$ II region we observed, yet none are as strong as those of the shocked regions we observed. No X-ray source from the catalogue of Long, Helfand and Grabelsky (1981) is situated in the vicinity of N179A, it is therefore unlikely to be a SNR. However, we would need 
Figure 2 Sample spectra in the wavelength region beyond $7000 \AA$ of $(a)$ the LMC supernova-remnant N49, (b) the Herbig-Haro object HH1, and (c) the LMC HIII region $\mathrm{N} 138 \mathrm{~A}$. 


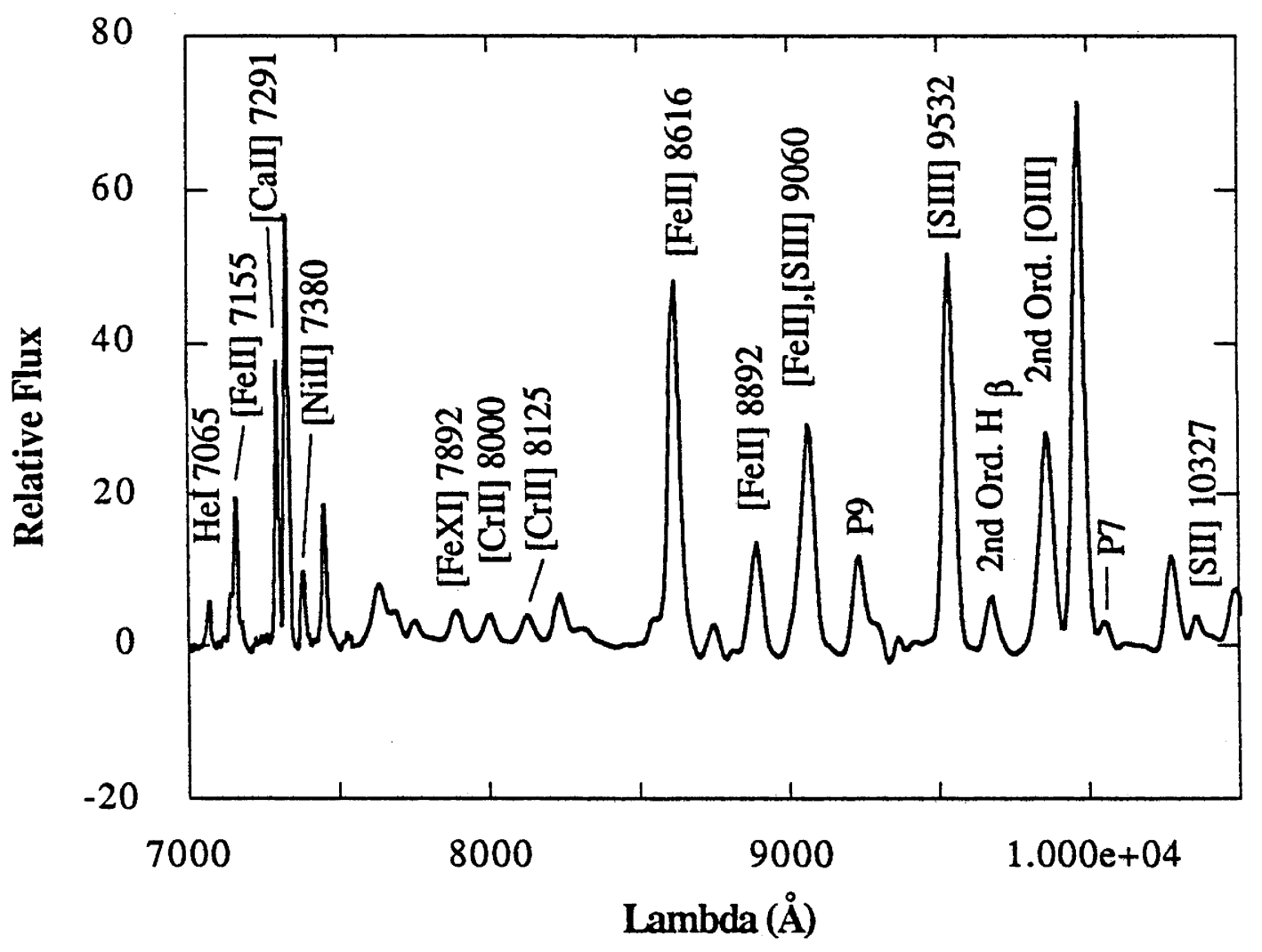




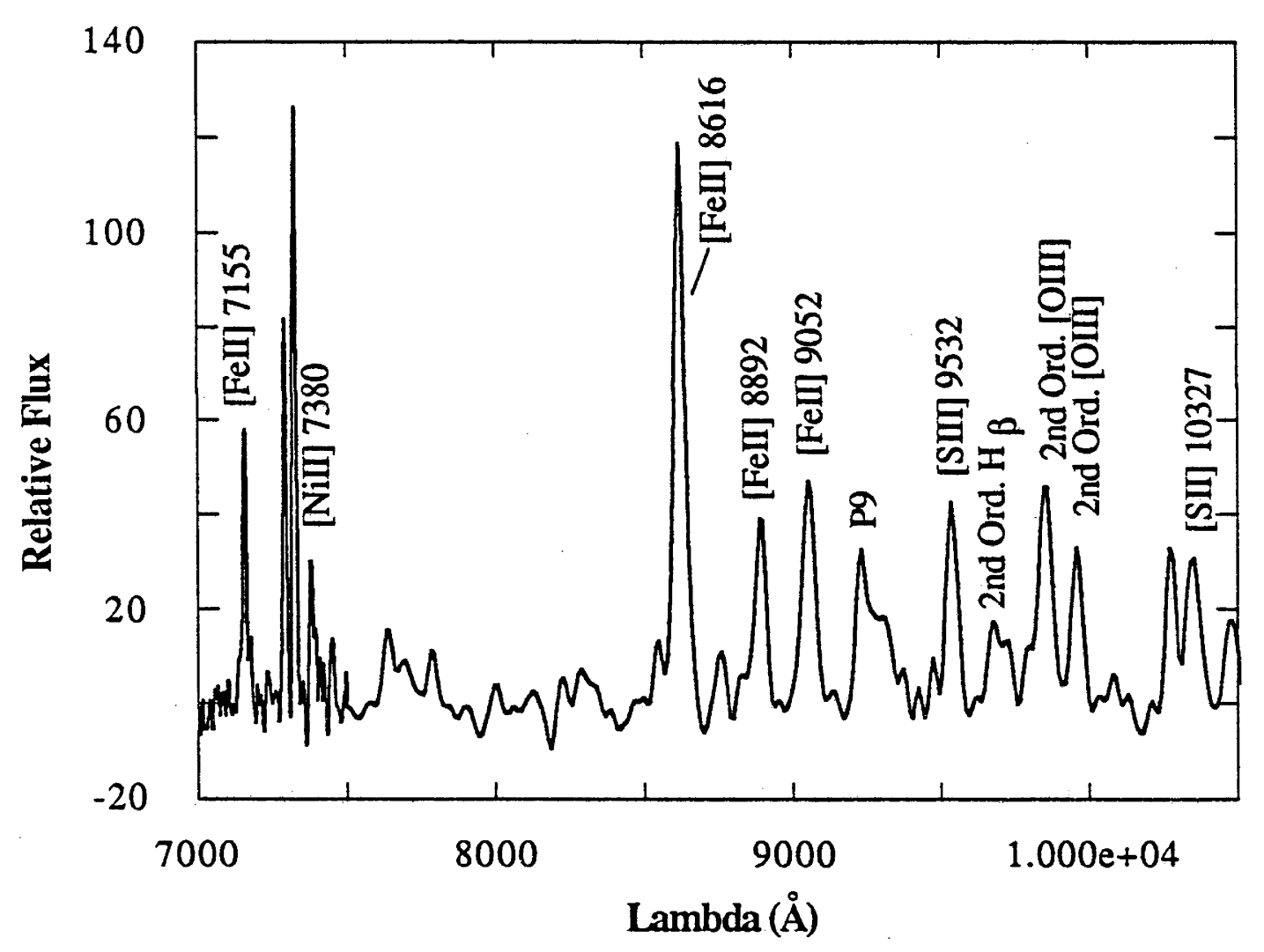




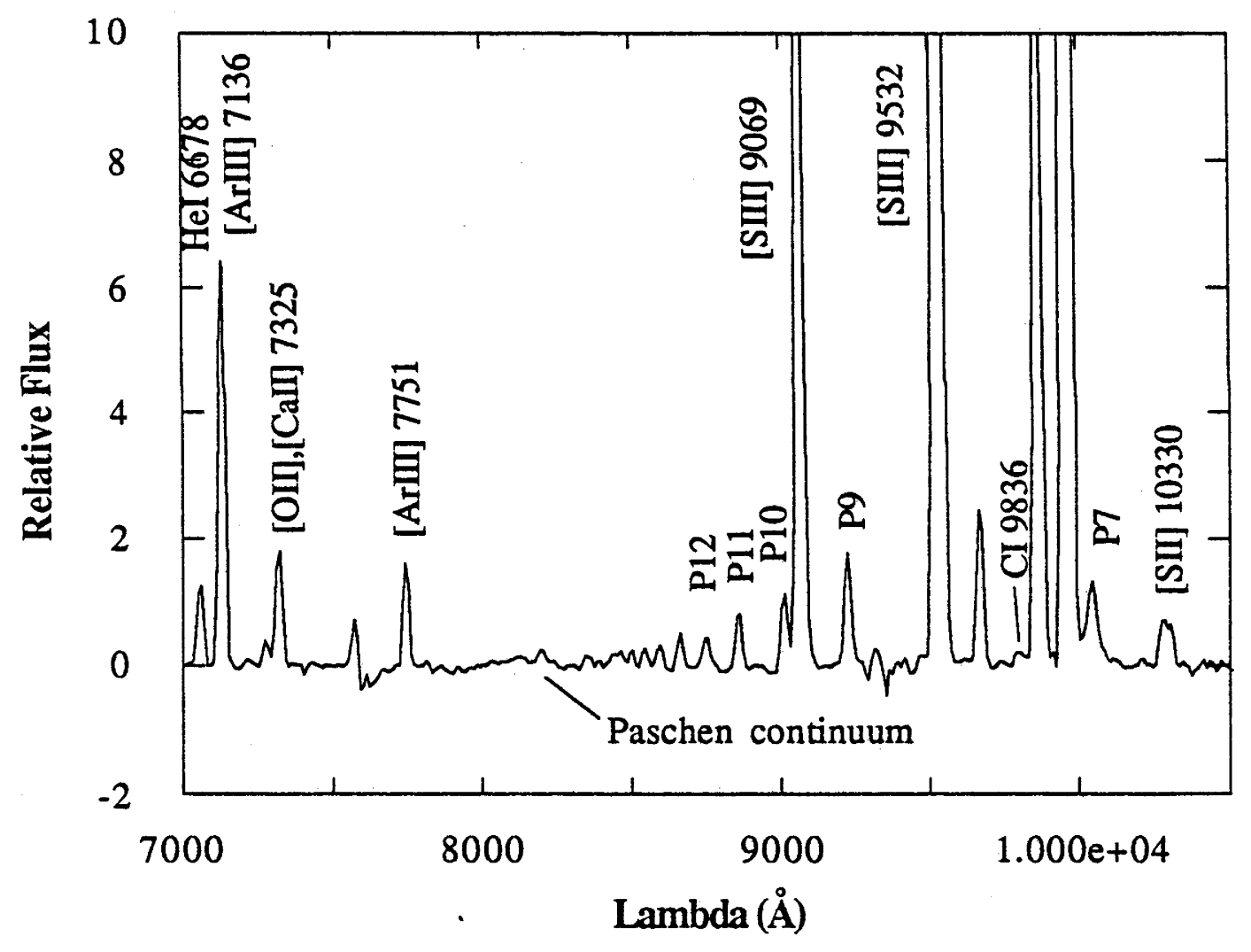


Figure 3 The optical spectra of (a) the SMC supernova-remnant SNR 0104-723, (b) the LMC HII region N84C, and (c) the very low excitation LMC HIIregion N179A. 


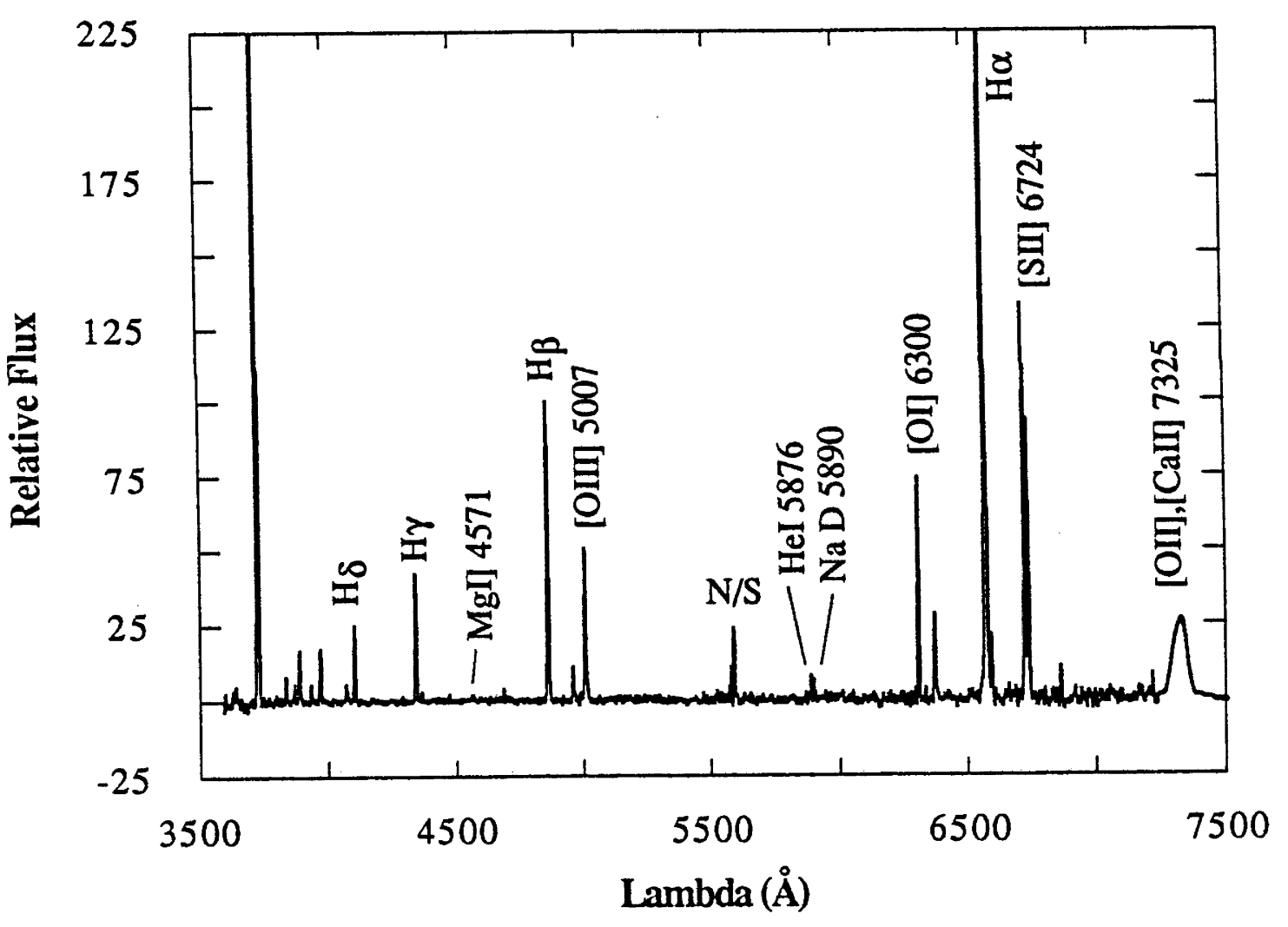




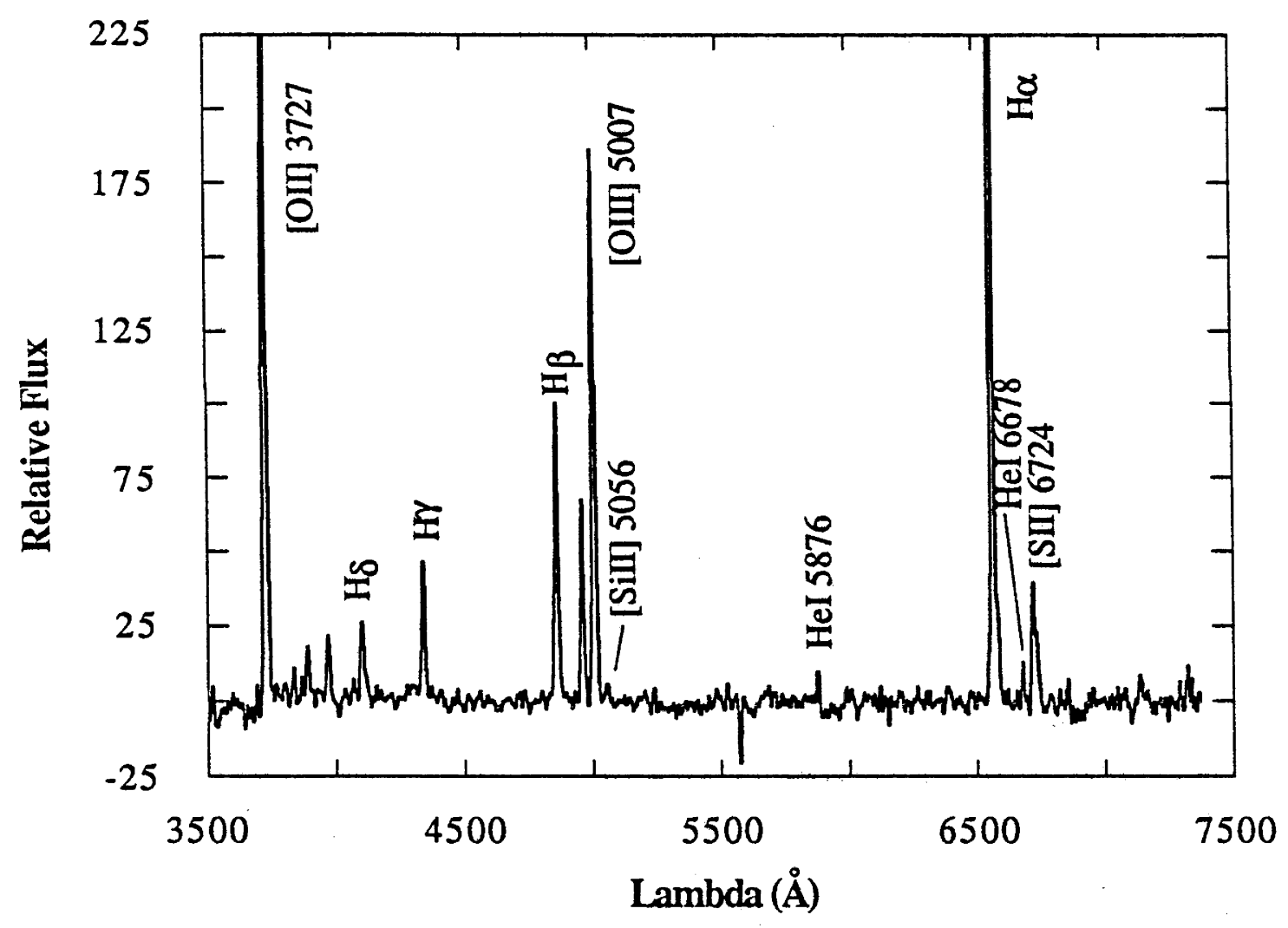




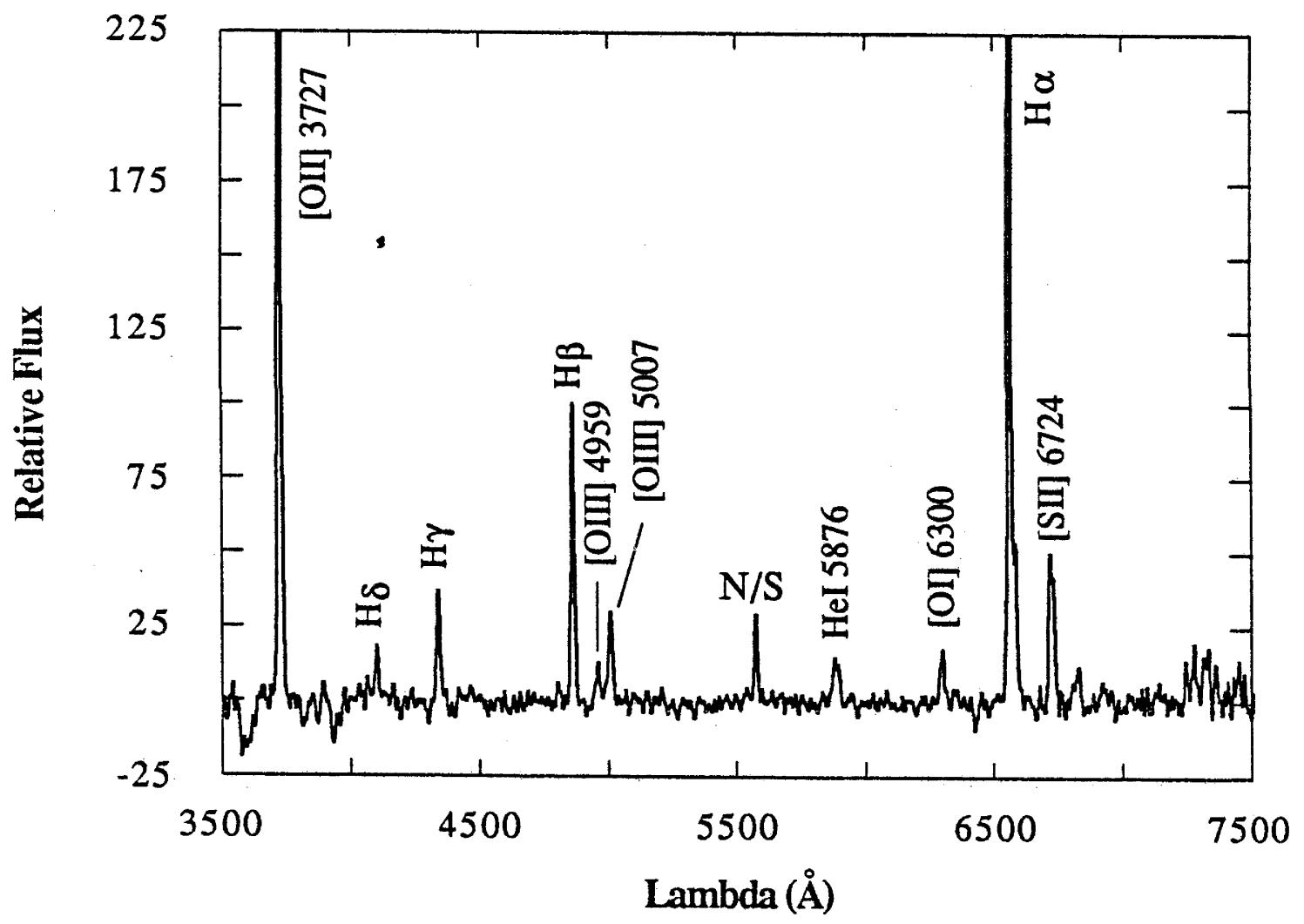


Figure 4 The spectrum of SNR0104-723 in the region of the Na D lines. 


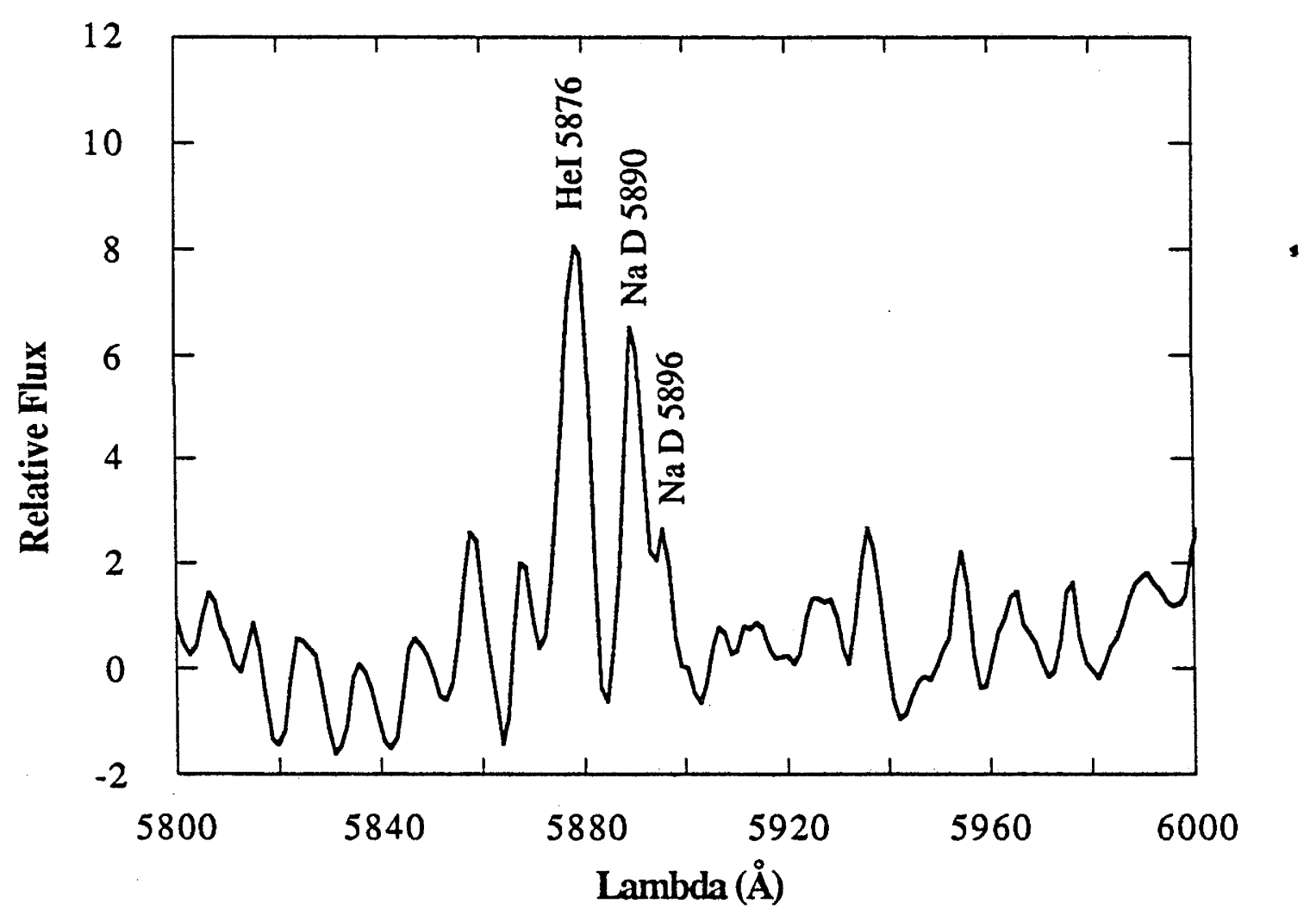


Figure 5 The spectrum of the SMC HII-region N12A in the region of the intercombination line $\mathrm{Mg}\rceil \lambda 4571$. 


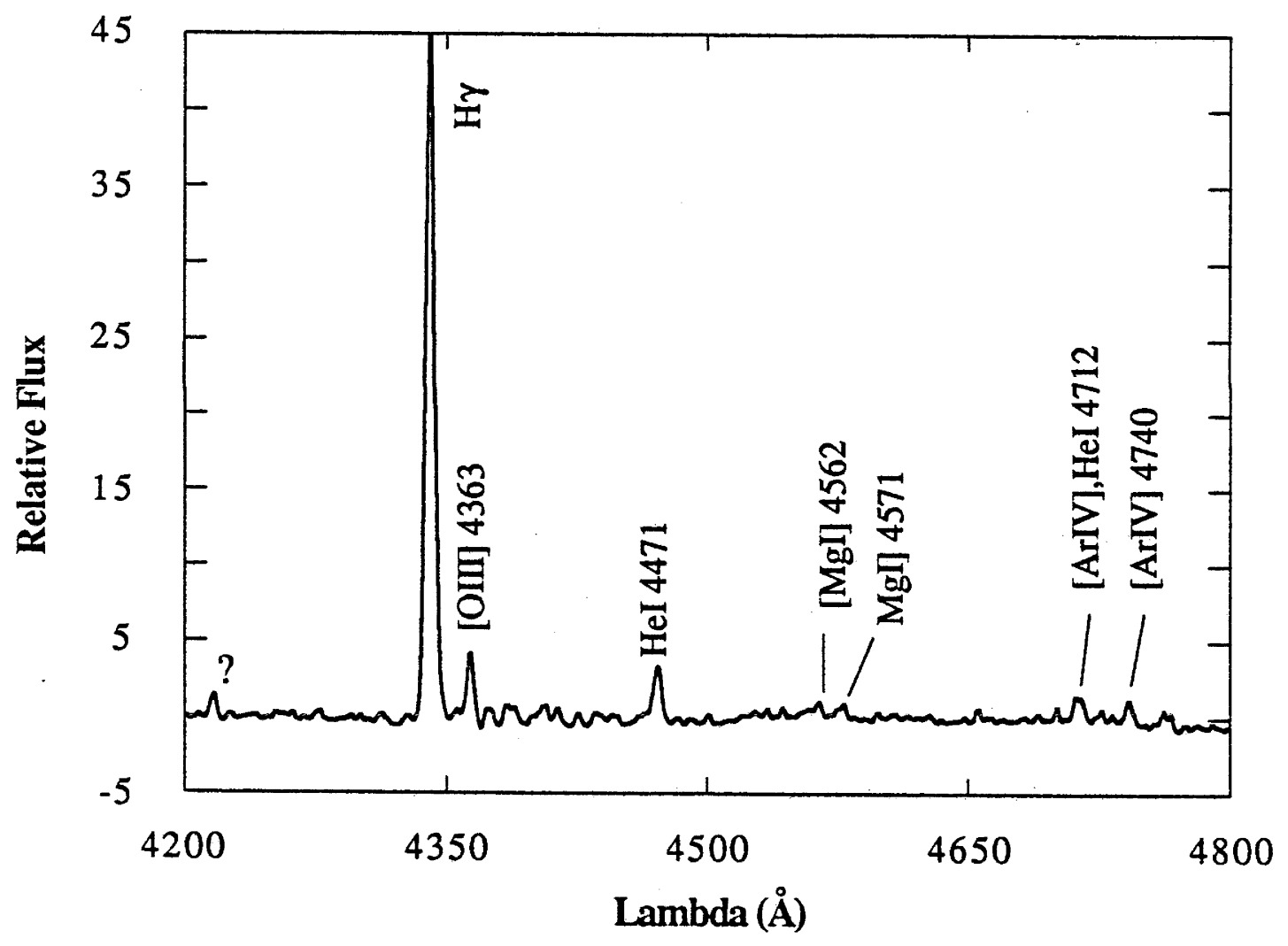


Figure 6 The spectrum of $\mathrm{N} 84 \mathrm{C}$ in the region of the $\mathrm{CaIl} \mathrm{H}$ and $\mathrm{K}$ lines. An error bar representing a three-sigma deviation from the mean in the continuum is included. 


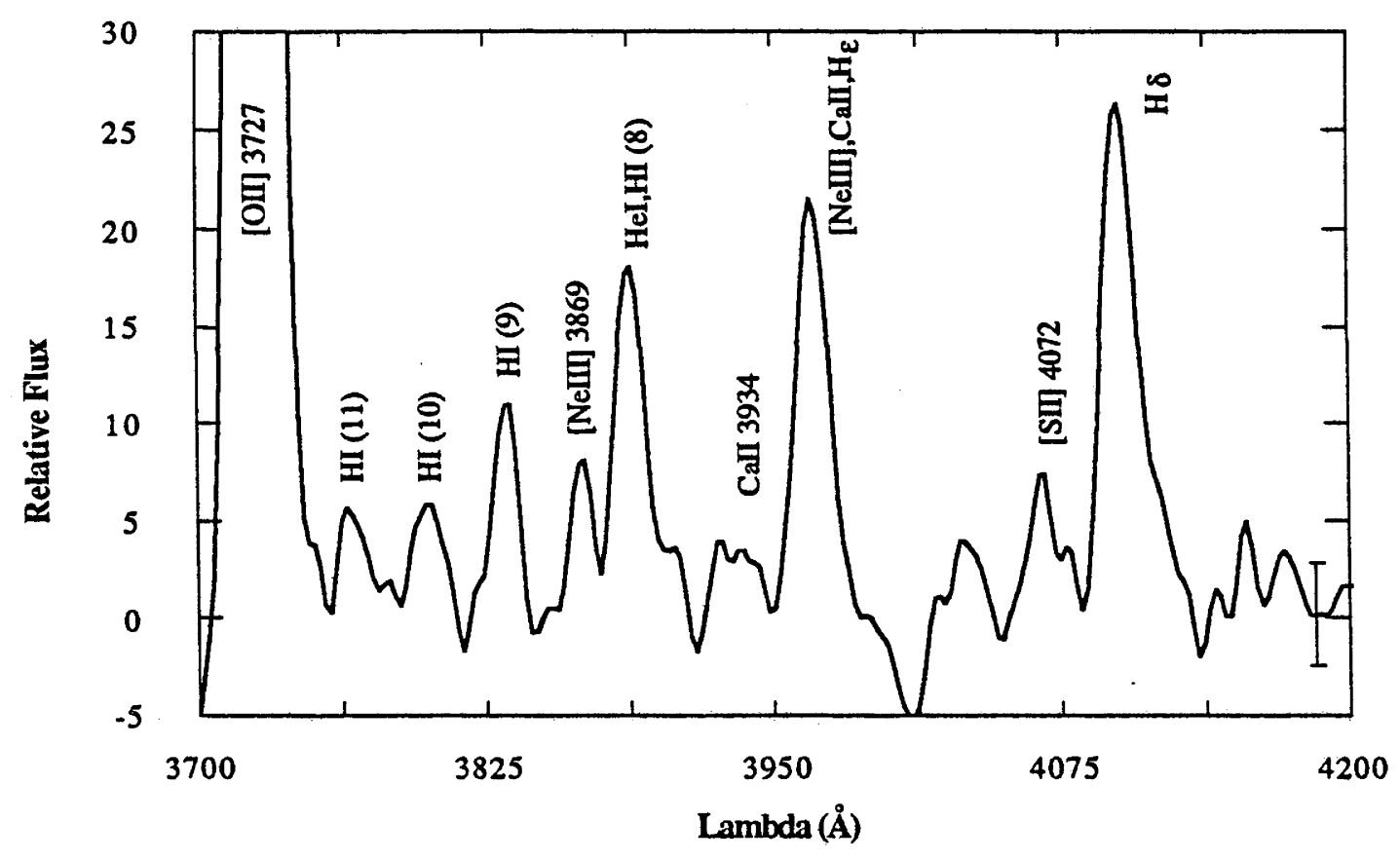


to take a high resolution $\mathrm{H} \alpha$-image of the region and make radio observations before we could be certain. It is more probable that it is the expanding, shock heated shell of a Wolf-Rayet (WR) star that we are observing, however this can not at present be confirmed since N179A lies just off the edge of the WR-star survey region of Azzopardi and Breysacher (1980). For the purposes of this paper therefore, N179A is treated as an ordinary $\mathrm{H} I I$ region, and modelled accordingly.

Of particular interest for us in the SNR spectra are the prominent lines of [CaII], [CrI], [FeII], [FeII] and [Nim], many of which are illustrated for the case of N49 in Figure $2 a$. This wealth of heavy metal lines allows us to link, for the first time, the abundance sets derived from $\mathrm{H}$ II regions and supergiants in the Magellanic Clouds. There are, however, several other lines of general interest, which include, for instance, the Na D doublet in the spectrum of SNR 0104-723, as shown in Figure 4. Care was taken to eliminate contamination of the prominent night-sky emission near this wavelength, so the detection of the line is not in doubt. It is highly unusual to observe this line due to its extremely low ionization energy $(\sim 5.1 \mathrm{eV})$, but it was detected recently in HH1 by Solf, Böhm and Raga (1988). Another low ionization stage $(\sim 7.6 \mathrm{eV})$ observed in shocked regions is $\mathrm{Mg} \Pi \lambda 4571$, was first observed by Herbig (1951). However it has never previously been detected in the spectra of an $\mathrm{H}$ II region to our knowledge. We have made a detection of this line in the SMC H II region N12A, which is illustrated here in Figure 5, along with the apparent detection of the forbidden line $[\mathrm{MgI} \lambda \lambda 4562$. With adequate modelling, this would allow an estimate to be made of the Mg depletion in this object. A convenient check on this could be made from the measurement of the line [SiII] $\lambda 5056$ which we marginally detected in the SMC H II region N84C, as illustrated in Figure $3 b$. Unfortunately, this line was not chosen for modeling in the MAPPINGS code. In the same object we were surprised to find that the subtraction of the model strength for $\mathrm{HI}(\varepsilon)$ from the line at $3968 \AA$, still left a line far too strong to be due only to [NeIII] $\lambda 3967$. Further investigation revealed the probable 
presence of the $\mathrm{Ca} \mathrm{K}$ line at $3934 \AA$ (see Fig. 6) with a strength in accord with that required by the $\mathrm{Ca} \mathrm{H}$ line to explain the strength of the blend. In addition, there is a possible detection of [CaI] $\lambda 7291$ and an increased strength in the blend at $7325 \AA$ due to [CaII] $\lambda 7324$. Apparently this object has an enhanced $\mathrm{Ca}$ abundance, which is probably the result of significant dust grain destruction in this object.

The line strengths were compared with those from the literature listed in Table 3, and scatter plots displaying the results are presented in Figures 7 to 10 . The first thing that may be remarked upon with regard to all these plots is that, for the most part (that is, excluding Fig. 10), there is no obvious systematic difference between our own dereddened line strengths, and those from other authors. This is interesting since we have, by and large, observed the integrated spectra of the whole of each object, while most observations reported in the literature are strictly at one slit position only. The only systematic differences between our results and those of other authors, are for the observations of $\mathrm{HH} 1$ made by Böhm and his colleagues shown in Figure 10a. This disparity is not apparent when we compare our own results for HHI, with those of other researchers (compare Fig. 9 (open circles), and $10 a)$. It is possible that the rather large bandwidth (20 $\AA$ ) of the scanner employed routinely by Böhm and his colleagues, included many faint emission lines which resulted in an over-estimate of the continuum level. However, in their most recent paper (Solf, Böhm and Raga, 1988), they used a resolution of $\sim 2 \AA$ (see Fig. 10b), and as we see from Figure $10 c$, the disparity is largely eliminated.

In detail, we note that there are several line strengths which deviate from the $45^{\circ}$ line quite markedly. The point marked " $a$ " in Figure $7 a$ is due to [SII $\lambda 6716$ from Pagel et al. (1978) for N127A, and is clearly a mismeasurement, for otherwise N127A is beyond the high density limit. Of more importance, are the deviant points in Figure 8, which are some crucial lines beyond $7000 \AA$, a spectral region for which there are few previous studies in the literature. The points labelled "a" - "d" 
Figure 7 A comparison between our line strengths for (a) LMC, and (b) SMC HII regions, and those from the literature. The lettered symbol is discussed in the text. The $45^{\circ}$ line is included for reference. 

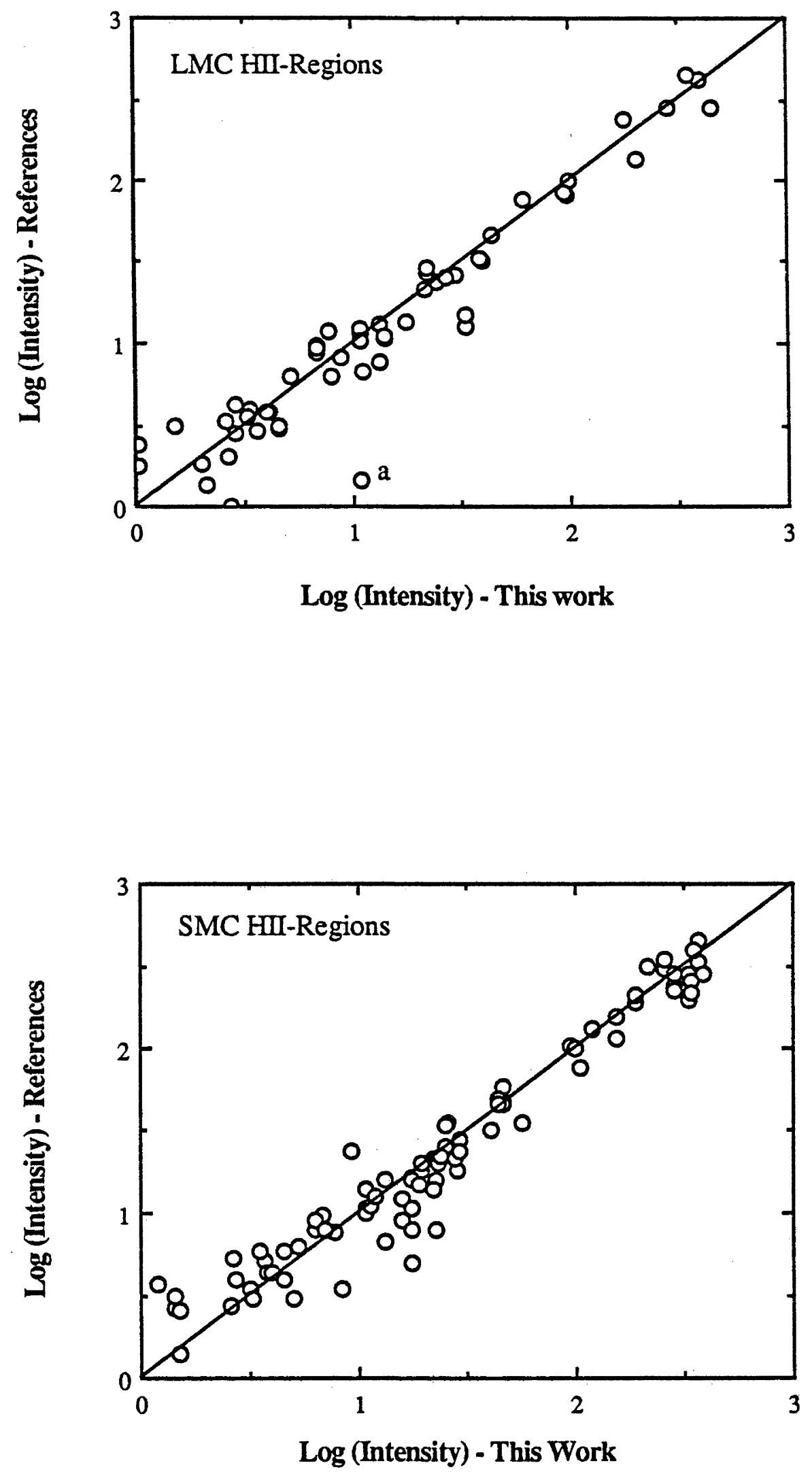
Figure 8 A comparison between our line strengths for SNRs in the Magellanic Clouds and those from the literature. The filled circles are derived from a comparison with Dennefeld (1986) for N63A, and the lettered symbols are discussed in the text. The $45^{\circ}$ line is included for reference. 


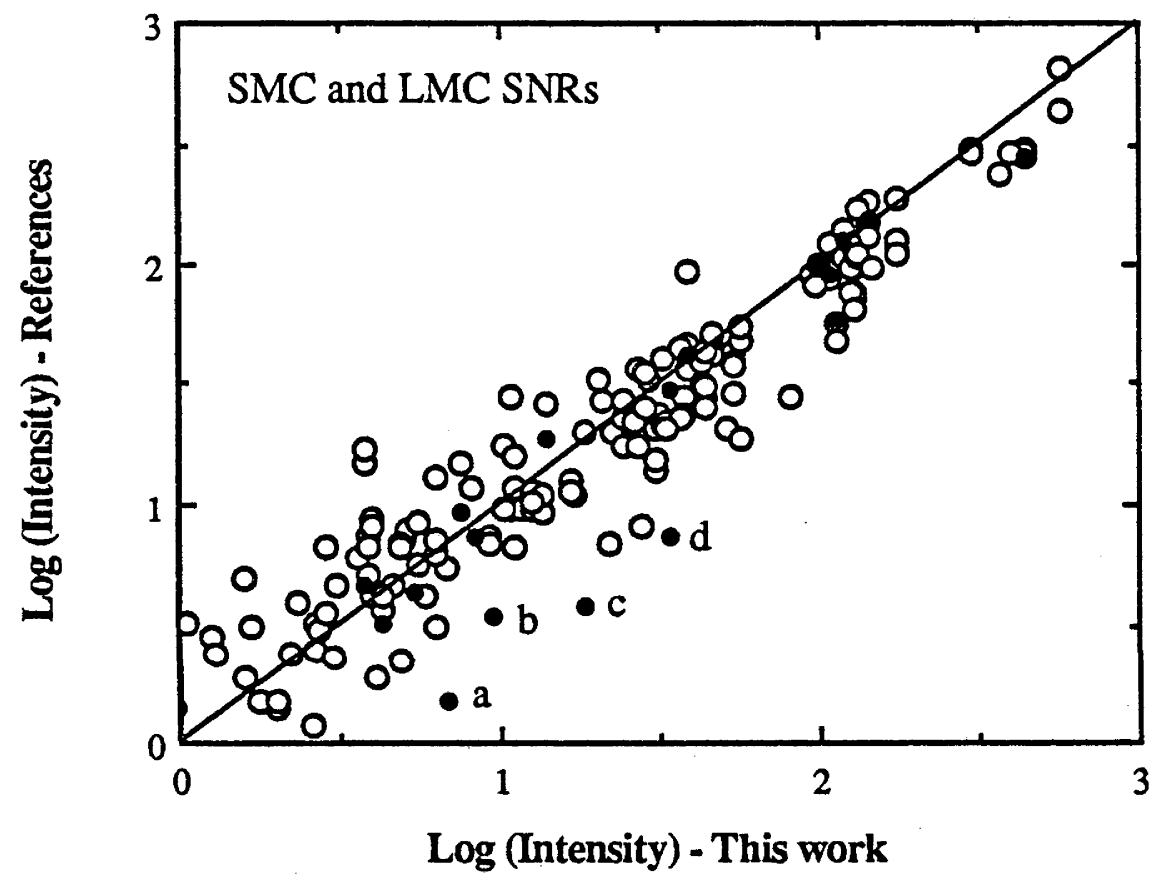


Figure 9 A comparison between our line strengths for Galactic Herbig-Haro objects and those from the literature (excluding the work of Böhm and his associates). The open circles represent $\mathrm{HH} 1$ and the filled circles are derived from the other $\mathrm{HH}$-objects. The $45^{\circ}$ line is included for reference. 


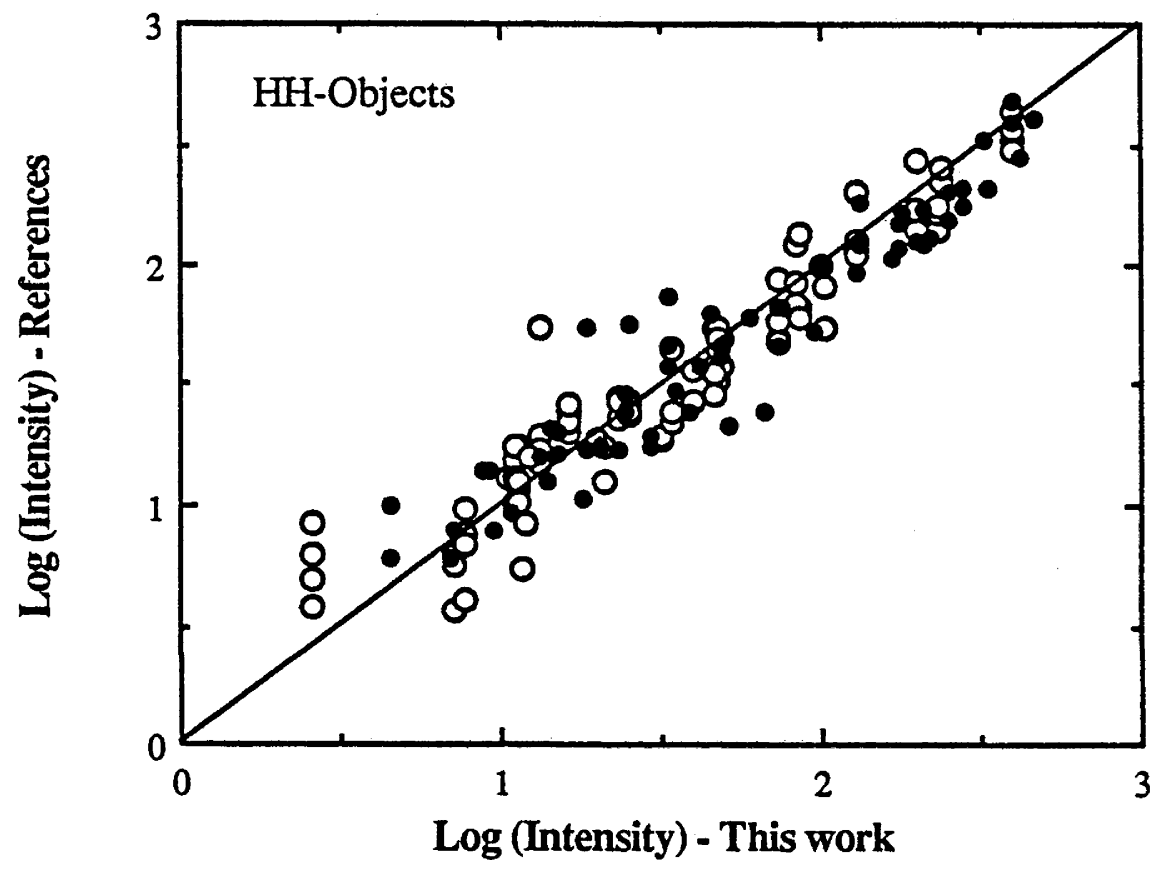


Figure 10a A comparison between our line strengths for $\mathrm{HH} 1$ and those from Böhm and his associates (excluding Solf, Böhm and Raga 1988). The $45^{\circ}$ line is included for referente.

Figure 10b A comparison between the line strengths of Solf, Böhm and Raga (1988) for HH1 and the previous work of Böhm and his associates.

Figure 10c A comparison between our line strengths for $\mathrm{HH} 1$ and those from Solf, Böhm and Raga (1988). 

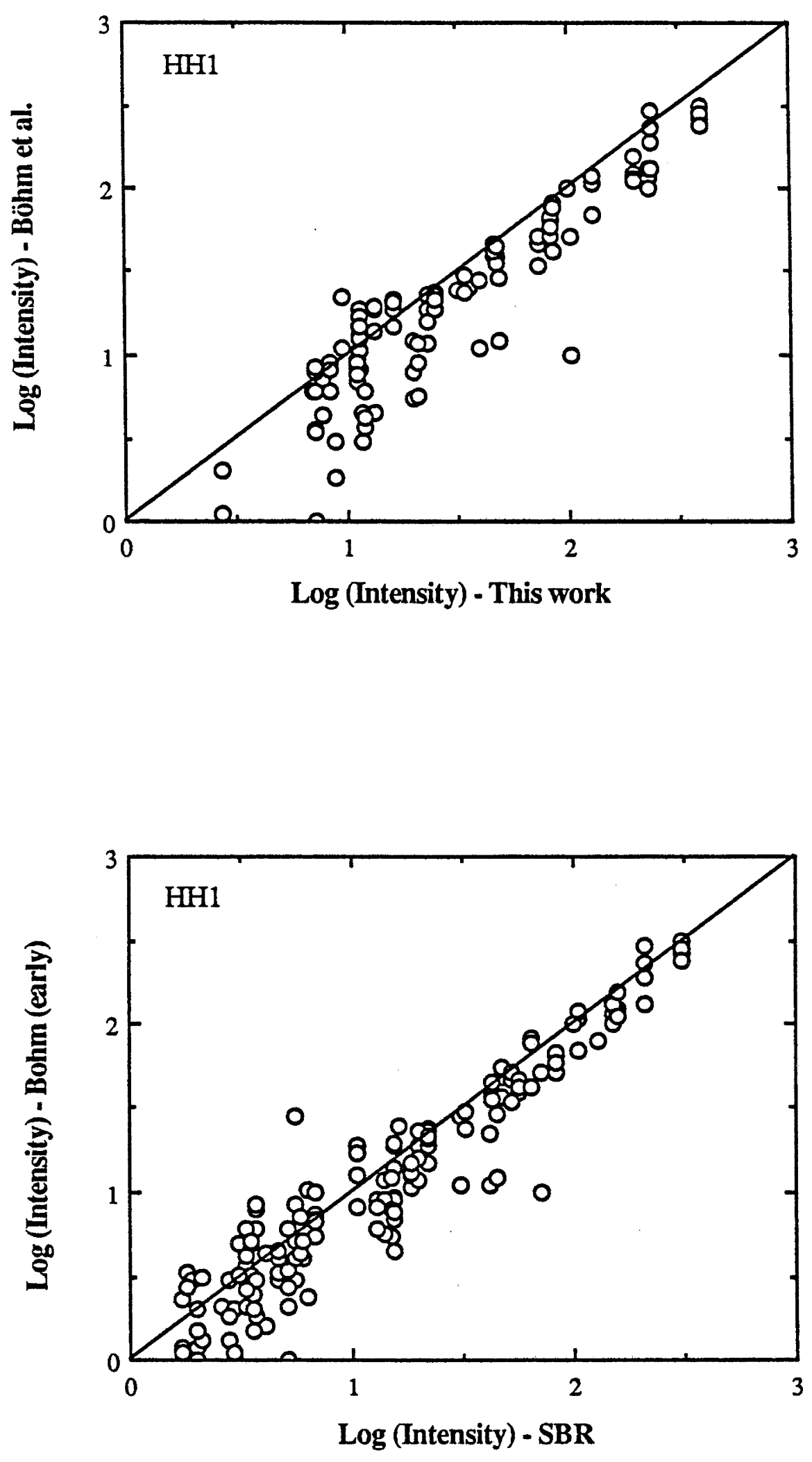


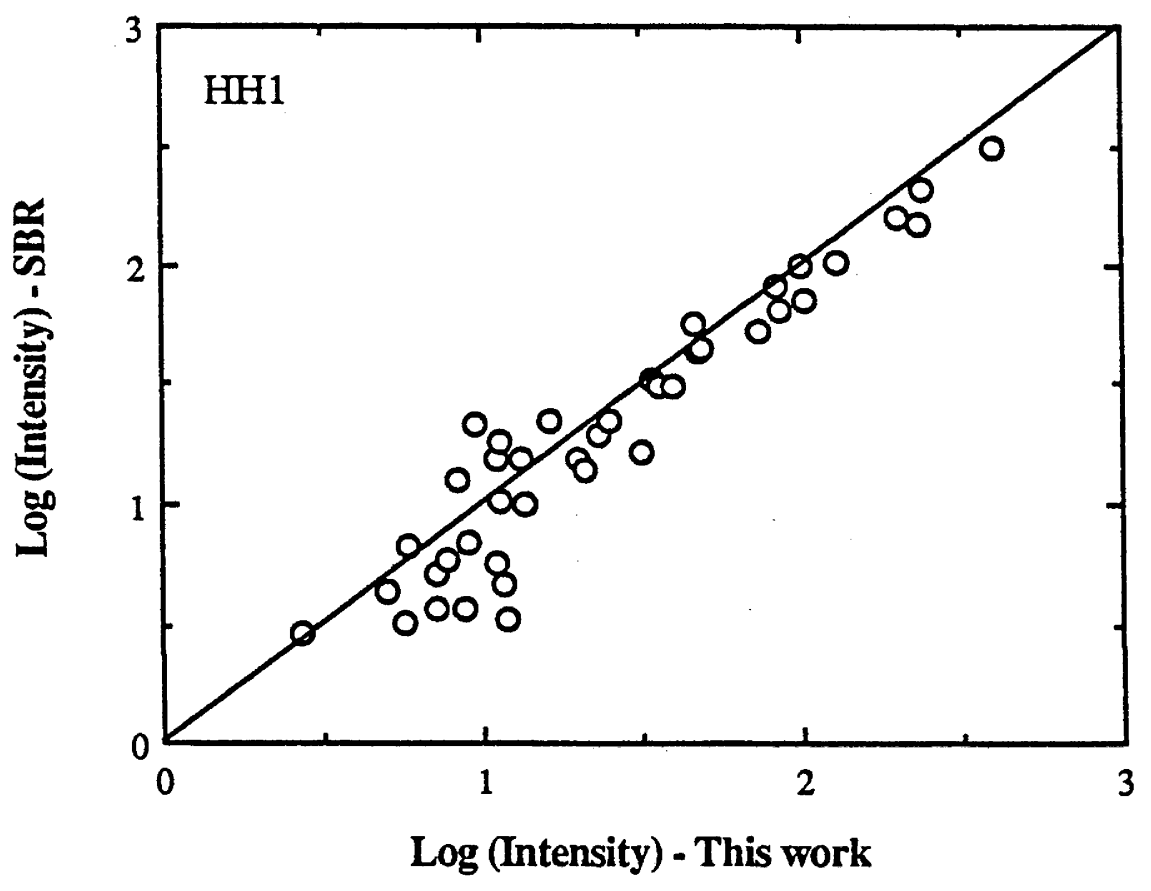


represent $[\mathrm{ArIII}] \lambda 7136,[\mathrm{FeI}] \lambda 7452,[\mathrm{SIII}] \lambda 9069$, and [SII] $\lambda 9532$ respectively, and are all derived from the same source in the literature (filled circles). In order to be sure that we are not affected by systematic error, we compared our estimates for the [SII] lines $\lambda 9069$ and $\lambda 9532$ with all similar measurements in the literature of objects in common with our own, and we found ours no more than $20-30 \%$ in excess on average. Since our only comparisons were between Dennefeld (1986), and Dennefeld and Stasinska (1983), and since we could expect large variations in this region of the spectrum due to atmospheric absorption, and differences in spatial sampling, we are probably doing well to achieve such closely correlated results. We also checked our measures of $\operatorname{Ar}[\mathrm{III}] \lambda 7136$ against those from other authors and found no sign of any systematic differences.

Due to the filamentary nature of SNRs there is a perennial problem in comparing observations of resolvable objects made at two different slit positions. This is seen most dramatically in comparisons [OII] and [OII] lines for the same objects as determined by different authors. A case in point is a comparison between the [OIII] lines derived from our own work for N63A and N103b, and the values derived from the literature. In the case of N63A we were careful to exclude the bright $\mathrm{H}$ II region to the West of the SNR, and still we measured more intense [OII] lines than in the literature (Danziger and Leibowitz 1985). An over-estimate of the sky subtraction due to contamination by the neighboring $\mathrm{H}$ II region could conceivably lower the [OIII] lines in particular. In support of our own measure for these lines, we can only add that our detailed models agree far better with the higher line-strengths than with the lower ones.

In the case of $\mathrm{N} 103 \mathrm{~b}$, however, we measured the [OII] line strengths to be significantly lower than in the literature. This is most probably due to our observing methods including a much greater proportion of low excitation emission over the bulk of the SNR than achieved with a single slit position on a bright filament. Thus the low excitation lines, including $H \beta$, are enhanced relative to the high excitation 
lines, effectively depressing the relative strength of the [OIM] lines. This may also be seen in the relative strengths of the [NeIII] $\lambda 3869 \AA$ lines, though not to such a large extent. The use of the [OII] lines, from integrated or single slit spectra as oxygen abundance indicators is therefore fraught with uncertainties, and can not be relied upon without additional information derived from other sources.

The accuracies of our line intensity measurements depend essentially upon the following factors:

(1) the quality of our sky subtraction,

(2) the accuracy of the flux calibration,

(3) photon statistics,

(4) the accuracy of continuum placement.

However, all contributions to the error should be reflected in the average scatter between our own measurements and those derived from the literature. Such a scatter would overestimate our own errors due to the variable quality of the spectra reported in the literature, and the fact that most of our spectra were integrated over the objects, while most studies from the literature used only single slit positions. Taking these effects into account, we estimate our own errors to be better than 0.07 to 0.1 dex over the majority of our spectra, but with increased errors for lines having a measured strength of the same order as $\langle\sigma\rangle$ (see Tables 4 and 5), and for lines in the following general areas:

a) 3727-3900 $\AA$ due to line crowding and a steeply changing continuum close to the Balmer limit;

b) 7000-7600 $\AA$ due to a dropping off in the IPCS detector sensitivity;

c) in the vicinity of the Paschen limit at $\sim 8200 \AA$;

d) >9600 $\AA$ due to line crowding (2nd order lines become important), a dropping off in the sensitivity of the CCD detector on FORS, and additional errors involved in correcting for the second-order blue leak. 


\section{THE PHYSICAL PARAMETERS H II REGIONS}

The ionization structure of an $\mathrm{H} I \mathrm{I}$ region is governed essentially by the mean hydrogen gas density $N$; a volume filling factor $\varepsilon$; the number of Lyman continuum photons, emitted per unit time from the central source, that are capable of ionizing neutral hydrogen, $L_{c}$; the oxygen abundance $\mathrm{N}(\mathrm{O}) / \mathrm{N}(\mathrm{H})$; and the ionization temperature of the central source $T_{\text {ion. }}$. Fortunately, the first three parameters can be

combined into a single mean ionization parameter $\bar{Q}$. This quantity is essentially a measure of the ratio of the mean number of ionizing photons crossing a unit area per unit time, to the mean number of hydrogen atoms per unit volume (thus having dimensions of velocity). In accordance with Evans and Dopita (1985), we define a volume weighted version of $\bar{Q}$ as follows:

$$
\bar{Q}=L_{C} /\left(4 \pi \bar{r}^{2} N\right), \quad \text { equation } 6 .
$$

where: $\quad \quad \quad r=\left(r_{\mathrm{e}}+R_{2}\right) / 2$,

$r_{\mathrm{e}}=$ radius of the empty zone as discussed by Dopita and Evans (1985),

$R_{2}=$ the effective Strömgren radius of the nebula, including the effect of the empty zone .

The spectrum of any nebula depends only upon the two parameters $T_{\text {ion }}$ and $\bar{Q}$, and on the abundances. The initial determinations for $T_{\text {ion }}$ and $\bar{Q}$ were made on the basis of the results from the three diagnostic diagrams, figures 11,12 and 17, of Evans and Dopita (1985), where we have assumed that the overall metallicities for each Cloud are $Z=1 / 4$ and $1 / 2$ solar for the SMC and LMC respectively. These diagrams make use of various ratios and combinations of [OI], [OII] and [OII] lines, and their relations with $\mathrm{H}_{\alpha}$ and $\mathrm{H} \beta$. In particular, all diagrams include some form of ratio with [OI] $\lambda 6300 \AA$ since this line is emitted in the transition zone of an 
H II region where there is an appreciable fraction of neutral Hydrogen. Unfortunately, there are very few measurements of the [OП $\lambda 6300 \AA$ line reported in the literature due to its relative weakness and the possibility of night-sky contamination at that wavelength. Our use of more sensitive detectors and our interest in objects where the doppler shift is sufficient to move the line away from the night-sky line, has allowed us to obtain good measures of this line in almost all cases. The averaged results are given in Table 6 with the average standard deviation from the mean of these three measures given in brackets. Also included in this table are the measures from an additional diagnostic diagram, figure 18 in Evans and Dopita, which are clearly discrepant in comparison with the other three diagnostics, and therefore excluded from the final mean values. This was unfortunate since, of all the diagnostic diagrams explored by Evans and Dopita, this one held the most promise. One of the diagnostics in this diagram depended upon the ratio of the [SII] lines at $\lambda 6731$ with that of the strong, but rarely observed, [SIII] line at $\lambda 9069 \AA$, hence implicating the [SII] line as the cause of the discrepancy. Such a problem has been reported previously in the literature by Evans (1986) and is most probably due to unreliable atomic data for the $\mathrm{S}^{++}$ion.

We attempted to determine the density of the $\mathrm{H}$ II regions from a comparison of the measured ratio of $[\mathrm{SH}] \lambda 6716 /[\mathrm{SI}] \lambda 6731$, to that modelled by us (see Fig. 11). However, this is a poor density diagnostic so close to the low density limit, so little reliance was placed on the results.

We were unable to obtain absolute $H(\beta)$ fluxes from our data due to the methods we used, however, we were able to provide adequate constraints on our models through estimates of the effective Strömgren radius, $R_{2}$ (see Table 6), derived from Davies, Elliott and Meaburn (1976) (DEM), and Henize (1956). With the physical parameters so determined, we were then able to proceed to the detailed modeling of each object, as described in the next section. 


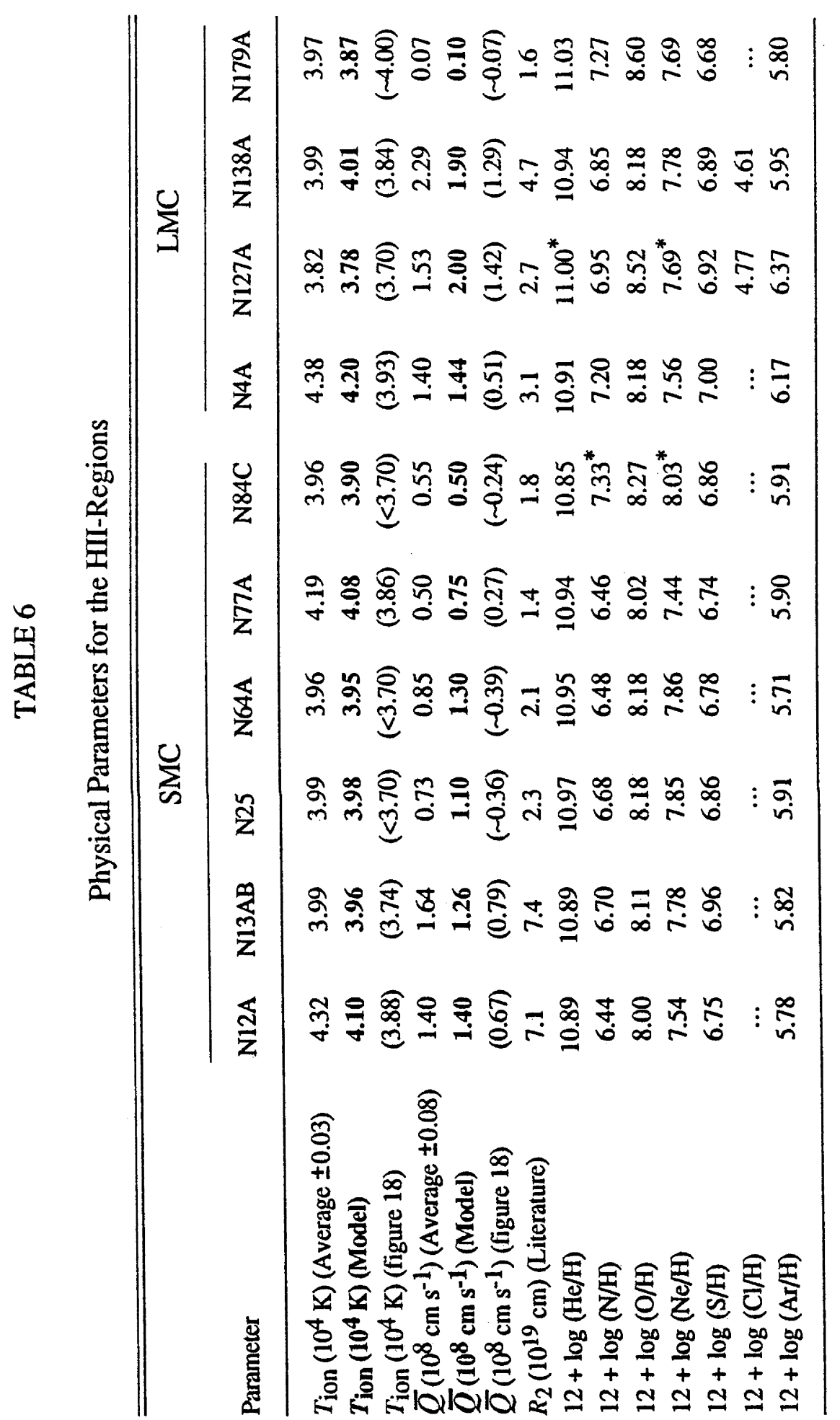


Figure 11 The $[\mathrm{SI}] \lambda 6716 / \lambda 6731$ ratio derived from our models, as a function of the of the electron density $\mathrm{Ne}$ for a gas at $10,000 \mathrm{~K}$ undergoing radiative cooling. 


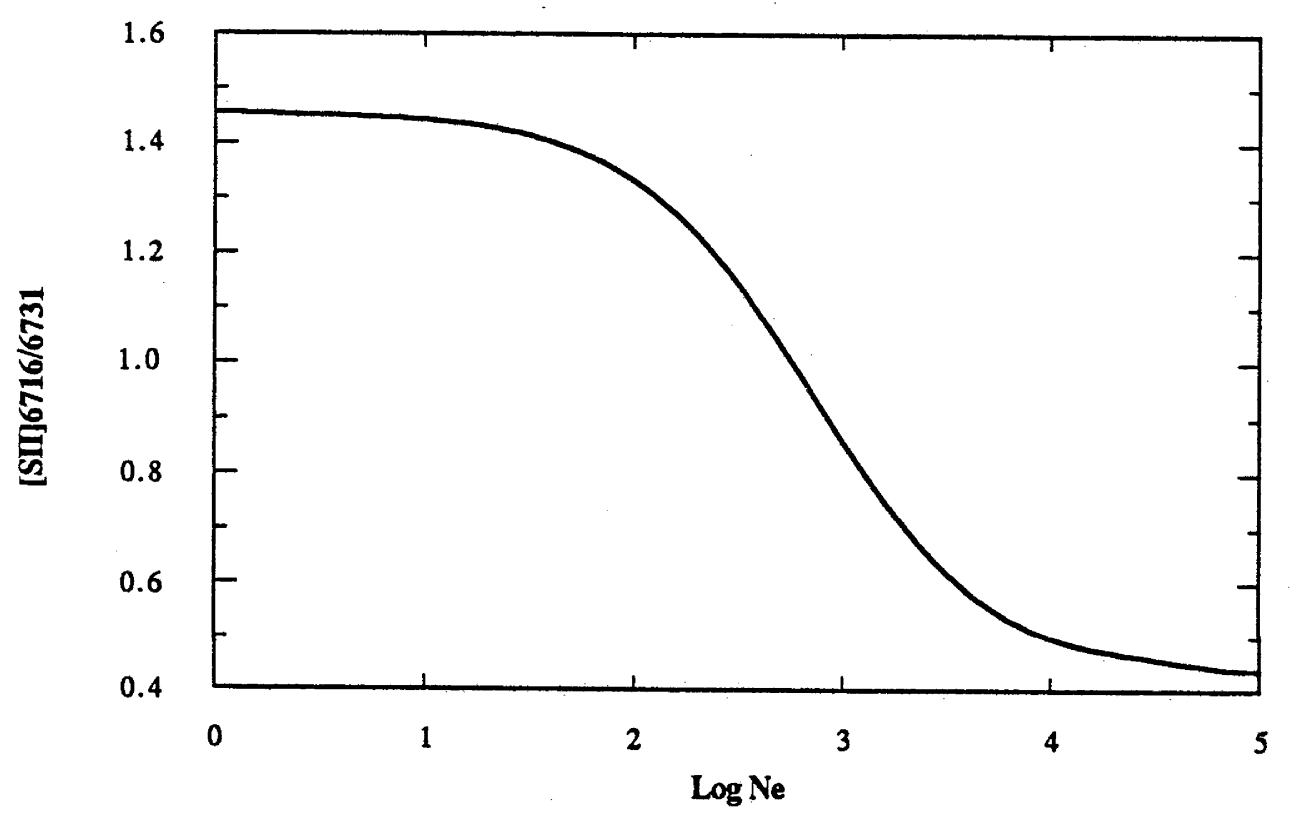




\section{THE DETAILED MODELING H II REGIONS}

The detailed models for each of the $\mathrm{H} I I$ regions were computed using the general-purpose modeling code MAPPINGS (mentioned in $\S I$ ), described in the paper by Evans and Dopita (1985), and the references cited therein. This code is not only used for modeling H II regions, but also the shock excited gases in SNRs, thus allowing us to obtain a consistent set of elemental abundances for both types of object.

In brief, each $\mathrm{H} \mathrm{II}$ region was modelled as a steady-state, spherically symmetric, dust-free nebula, consisting of infinitesimal filaments of gas with a volume filling factor $\varepsilon$. Radiative transition rates and collision strengths employed in calculating the forbidden-line intensities and associated cooling are taken from a paper by Mendoza (1983) and references cited therein. The code incorporates 12 elements with up to six ionization stages each. An ion is treated as a five level system, and the forbidden line intensities computed by solving for statistical equilibrium between the metastable states. The central $O B$ association was approximated by a single source of ionizing photons, with a stellar atmosphere having an ionization temperature $T_{\text {ion }}$ (which will be close to the temperature of the hottest star in the cluster due to the high temperature dependence of the photon flux (see Searle, 1971). Using the interpolation technique described by Shields and Searle (1978), the atmosphere was derived from the $\log g=4.0$ LTE-models of Hummer and Mihalas (1970). It is important to note here that in the calculation of the metal edges, use was made of the non-LTE models of Auer and Mihalas (1972) where necessary (as described in Shields and Searle 1978). Since the atmospheric blanketing of a star is heavily dependant upon the metal abundance (see Balick and Sneden 1976, Borsenberger and Stasinska 1982, and Koppen, Schmid-Burgh and Scholz 1983), the gas+dust abundance of the nebula was constrained to match that 
of the stellar atmosphere. In order to simplify the computations involved in rigorous 2-phase models (see for instance Stasinska, 1980 and 1982), the central source is assumed to be surrounded by an empty zone of radius $r_{\mathrm{e}}$ as discussed by Evans and Dopita (1985).

The initial estimates for the physical parameters, as discussed in the previous section, were set up in MAPPINGS, and a best fit model was calculated through a process of trial and error, while keeping the effective Strömgren radius $R_{2}$ constant . Convergence was sought first for the set of line ratios (3727.3727/4861.6563) versus (6300.6300/3727.6563) (fig. 17 in Evans and Dopita 1985). This allowed us to fine-tune the values for $\bar{Q}$ and $T_{\text {ion. }}$. In the second instance, we fine-tuned the oxygen abundances by ensuring that the line strengths of [OI] $\lambda 6300$, [OII] $\lambda 3727$ and [OII] $\lambda 5007$ were matched to the observations. Finally, the abundances of the elements $\mathrm{He}, \mathrm{N}, \mathrm{Ne}, \mathrm{S}, \mathrm{Cl}$ and $\mathrm{Ar}$ were adjusted to match the observed line strengths.

Although the measures of $R_{2}$, were not expected to be of great accuracy, when we increased one radius by over $50 \%$ and reconverged our model, we found no change in the derived abundances. Thus, although we can set poor constraints on the physical structure of the nebulae, we are confident that the quality of the abundances we have derived are limited mainly by the observational uncertainties in the line strengths.

The finally adopted model parameters are listed in bold type in Table 6 for each object, and a comparison between the model and observed line ratios, is given in Table 7. In the latter table those lines for which a match was demanded are shown in italics. In order to estimate the strength of the HeI line at $3889 \AA$, the model strength of 10.51 for $\mathrm{HI}(8)$, was subtracted from the blend. Similarly, for the blend at $3969 \AA$, we assumed a negligible strength for the CaII line, and a theoretical strength of 15.91 for $\mathrm{HI}(\varepsilon)$. As discussed in $\S \mathrm{II} \mathrm{c}$, this was satisfactory for all except N84C, where the Call line was found to be significant. In the same 


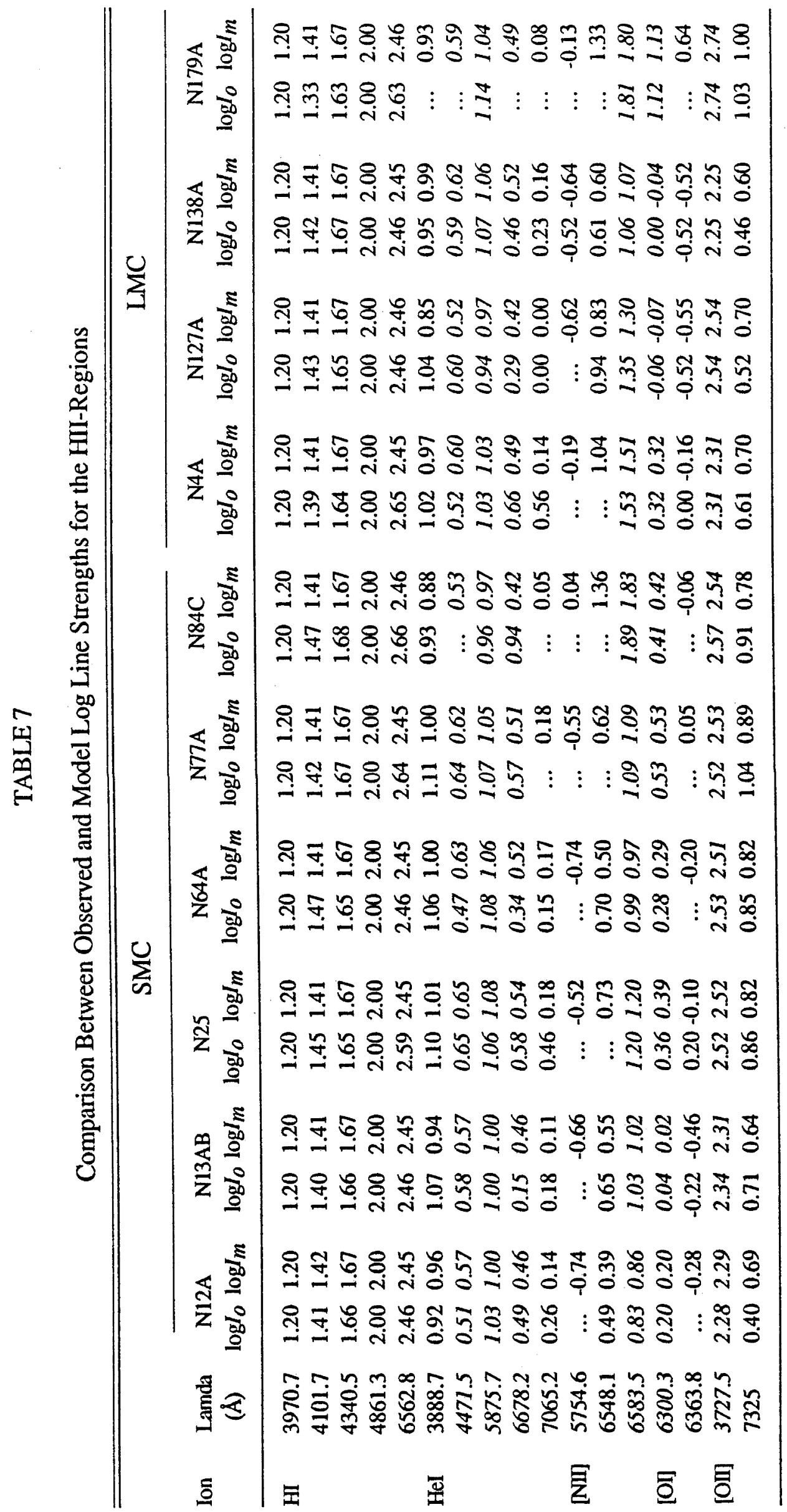




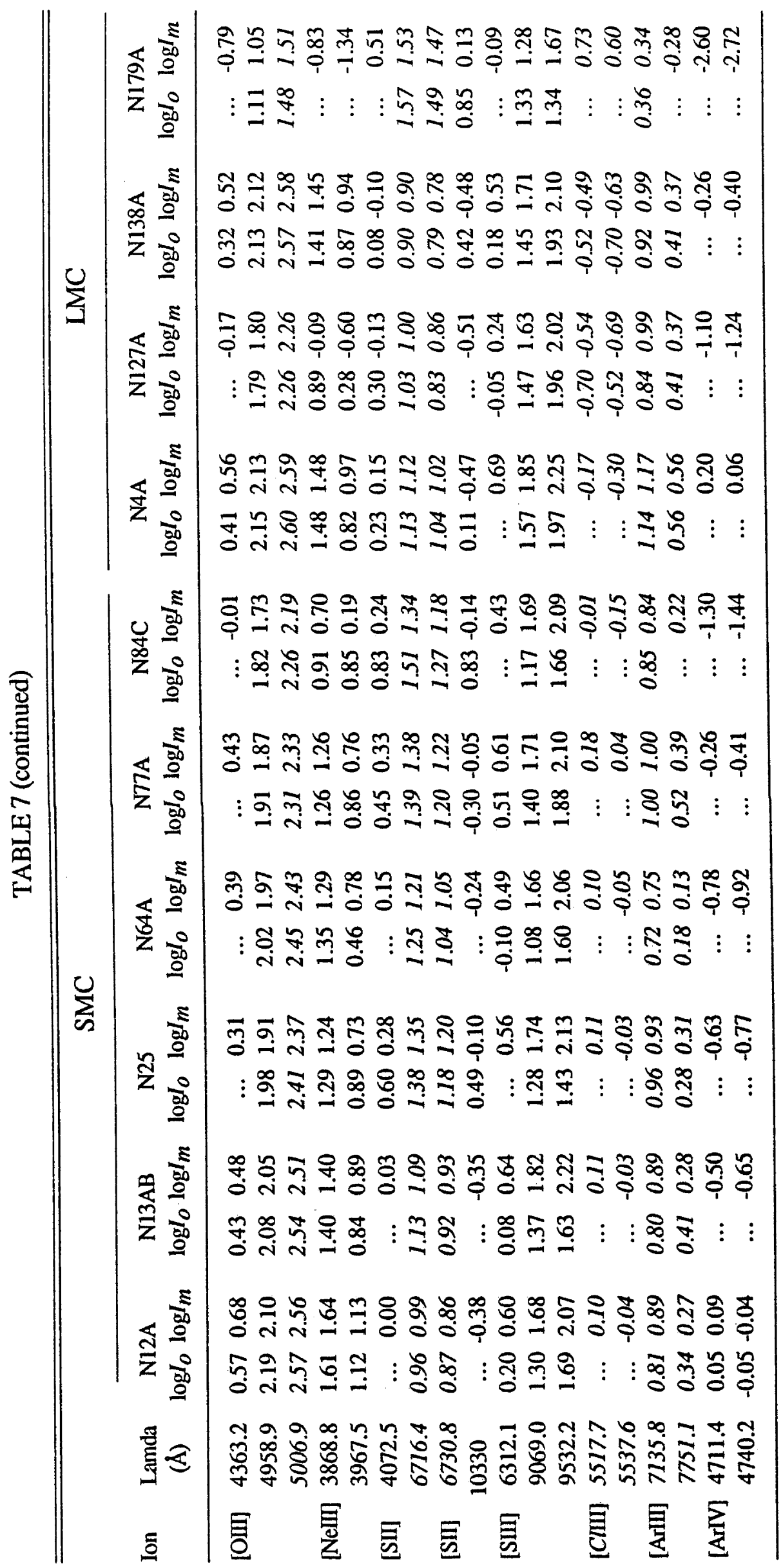


object, the [CaIl] line strength at $7324 \AA$ was taken to be $2 / 3$ of $[\mathrm{CaII}] \lambda 7291$, and subtracted from the blend to derive the combined [OM] line strength. For all other cases, [CaII] $\lambda 7324$ was assumed to be zero. For the blend between the lines [SIII] $\lambda 9532$ and $\mathrm{HI}(\mathrm{P} 8) \lambda 9546$, we subtracted the theoretical strength of $\mathrm{HI}(\mathrm{P} 8)=3.6$ to obtain the $[\mathrm{SII}]$ line.

The average elemental abundances for each Cloud, both from our own work and from the literature, are shown in Table 8 . Included in this table is $\sigma$, the standard deviation from the mean abundance within our samples, which include the effects of random error in our abundance estimates, and intrinsic variation in the abundances of the studied regions. We display these results graphically in Figures $12 a$ and $12 b$, in terms of the differences between the abundances in the Clouds and the Sun (as listed at the bottom of Table 8). For illustrative purposes, we have included in these figures a dashed line representing the mean $\mathrm{Fe}$ deficiencies for each Cloud, as derived in Paper I. The details of these results are discussed in the following section. 
TABLE 8

Average HII-Region Elemental Abundances in Terms of $12+\log \mathrm{N}(\mathrm{X} / \mathrm{H})$

\begin{tabular}{|c|c|c|c|c|c|c|c|c|c|}
\hline Reference & Number & $\mathrm{He}$ & $\mathrm{C}$ & $\mathbf{N}$ & 0 & $\mathrm{Ne}$ & $S$ & $\mathrm{Cl}$ & Ar \\
\hline \multicolumn{10}{|c|}{ SMC } \\
\hline Dufour et al. 1984 & 2 & $\ldots$ & 7.16 & 6.46 & 8.02 & $\ldots$ & $\ldots$ & $\ldots$ & $\ldots$ \\
\hline Dennefeld \& Stasinska 1983 & 6 & $\cdots$ & $\ldots$ & $\ldots$ & 8.02 & $\ldots$ & 6.26 & $\cdots$ & $\ldots$ \\
\hline Dufour et al. 1982 & 3 & $\ldots$ & 7.16 & 6.60 & 8.05 & 7.34 & 6.61 & $\ldots$ & 5.77 \\
\hline Aller et al. 1979 & 6 & $\ldots$ & $\ldots$ & 6.45 & 8.10 & 7.58 & 6.29 & $\ldots$ & 5.86 \\
\hline Pagel et al. 1978 & 23 & $\ldots$ & $\ldots$ & 6.41 & 7.98 & 7.16 & 6.38 & 4.48 & 5.94 \\
\hline Dufour \& Harlow 1977 & 101 & 10.90 & $\ldots$ & 6.48 & 8.02 & 7.29 & 6.40 & $\ldots$ & $\ldots$ \\
\hline Dufour \& Killen 1977 & 31 & 10.87 & $\ldots$ & 6.26 & 8.02 & 7.19 & $\ldots$ & $\ldots$ & $\ldots$ \\
\hline Peimbert \& Torres-Peimbrt 1976 & 31 & 10.89 & $\ldots$ & 6.48 & 8.05 & 7.30 & $\ldots$ & $\ldots$ & $\ldots$ \\
\hline Webster 1976 & 61 & 10.95 & $\ldots$ & $\ldots$ & 7.73 & $\ldots$ & $\ldots$ & $\ldots$ & $\ldots$ \\
\hline Dufour 1975 & 31 & 10.97 & $\ldots$ & 6.49 & 8.05 & 7.18 & 7.15 & $\ldots$ & 7.02 \\
\hline Aller et al. 1974 & 10 & 11.00 & $\cdots$ & 6.28 & 7.97 & 7.40 & 6.5 & $\cdots$ & $\cdots$ \\
\hline Dufour 1984 (review) & & 10.90 & 7.16 & 6.46 & 8.02 & 7.22 & 6.49 & 4.70 & 5.78 \\
\hline This work & & 10.92 & $\cdots$ & 6.55 & 8.13 & 7.69 & 6.83 & $\ldots$ & 5.84 \\
\hline$\sigma$ & $(0.05$ & & $\ldots$ • & $(0.13)$ & $(0.10)$ & $(0.19)$ & $(0.08)$ & $\cdots$ & $(0.08)$ \\
\hline \multicolumn{10}{|c|}{ LMC } \\
\hline Dufour et al. 1984 & & $\ldots$ & 7.90 & 6.97 & 8.43 & $\ldots$ & $\ldots$ & $\ldots$ & $\ldots$ \\
\hline Dennefeld \& Stasinska 1983 & 10 & $\ldots$ & $\ldots$ & $\ldots$ & 8.42 & $\ldots$ & 6.67 & $\ldots$ & $\ldots$ \\
\hline Dufour et al. 1982 & 4 & $\ldots$ & 7.90 & 6.94 & 8.38 & 7.68 & 7.01 & $\ldots$ & 6.10 \\
\hline Aller et al. 1979 & 16 & ... & $\ldots$ & 7.02 & 8.43 & 7.77 & 6.90 & $\ldots$ & 6.35 \\
\hline Pagel et al. 1978 & & $\ldots$ & $\ldots$ & 6.88 & 8.39 & 7.61 & 6.79 & 4.89 & 6.35 \\
\hline Webster 1976 & 21 & 11.00 & $\ldots$ & $\ldots$ & 7.71 & $\ldots$ & $\ldots$ & $\ldots$ & $\ldots$ \\
\hline Dufour 1975 & 111 & 11.01 & $\ldots$ & 6.80 & 8.43 & 7.64 & 7.15 & 5.01 & 7.10 \\
\hline Aller et al. 1974 & 201 & 10.99 & $\ldots$ & 6.94 & 8.46 & 7.83 & 7.2 & $\ldots$ & $\ldots$ \\
\hline Peimbert \& Torres-Peimbert 1974 & 41 & 10.92 & $\ldots$ & 7.10 & 8.58 & 7.94 & $\ldots$ & ... & $\ldots$ \\
\hline Dufour 1984 (review) & & 10.93 & 7.90 & 6.97 & 8.43 & 7.64 & 6.85 & 4.84 & 6.20 \\
\hline This work & & 10.96 & $\cdots$ & 7.07 & 8.37 & 7.68 & 6.87 & 4.69 & 6.07 \\
\hline$\sigma$ & $(0.06$ & & $\ldots$ & $(0.20)$ & $(0.22)$ & $(0.11)$ & $(0.14)$ & $(0.11)$ & $(0.25)$ \\
\hline \multicolumn{10}{|c|}{ Galactic } \\
\hline Orion - HII (Dufour et al. 1982 ) & & 10.97 & 8.45 & 7.48 & 8.59 & 7.80 & 7.12 & 5.16 & 6.27 \\
\hline Solar vicinity - HII (Shaver et al. 1 & $1983)$ & 11.0 & $\ldots$ & 7.57 & 8.70 & 7.9 & 7.06 & $\ldots$ & 6.42 \\
\hline Sun (Cameron 1982) & & $\ldots$ & 8.62 & 7.94 & 8.84 & 7.99 & 7.27 & 5.25 & 6.60 \\
\hline
\end{tabular}


Figure 12 The abundance distribution of the elements in the HII-regions we studied in (a) the LMC, and (b) the SMC, with respect to the Sun. The filled circles represent our own results with error bars representing the object-to-object scatter. The open circles represent the results derived from the literature, and the dashed line represents the Fe deficiency for the Cloud, as derived in paper I. 

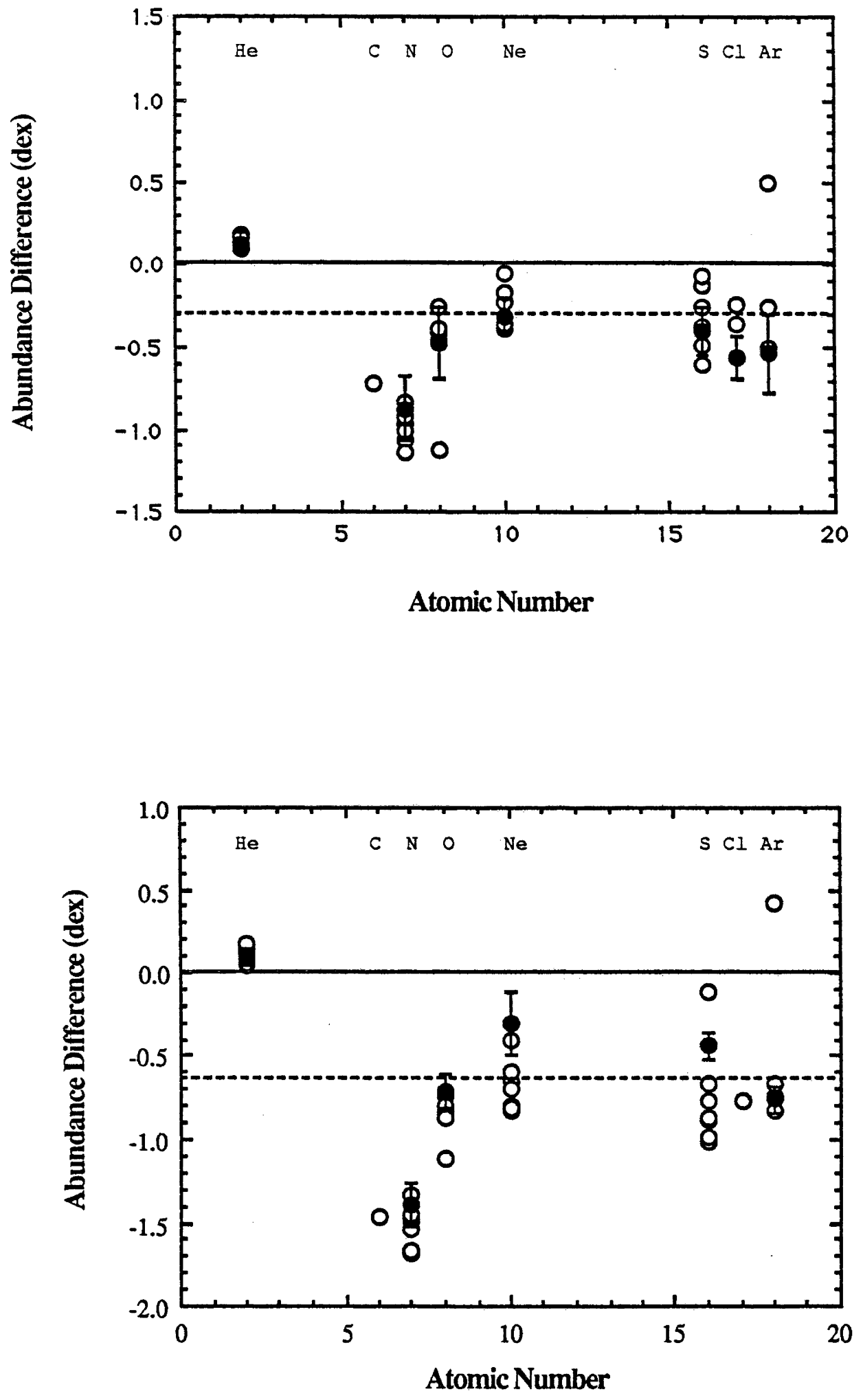


\title{
V.RESULTS \\ HII REGIONS
}

\author{
a) Helium
}

In all of the objects we studied, we were able to obtain good measures for the strong HeI triplet line at $5876 \AA$, plus, in most cases, measures for the triplet line at $4472 \AA$ and the singlet line at $6678 \AA$. We used these three lines as discriminants in our models, weighting them with a ratio of $3: 1: 1$. The singlet line at $6678 \AA$ was not always reliable, however, and in these cases, only the lines at $5876 \AA$ and $4472 \AA$ were used as discriminants.

The line at $7065 \AA$ is predicted to be systematically weak by our models, but this was also observed for the three nebulae in M101 modelled by Evans (1986), and explained as being due to resonance fluorescence by self-absorption of $\lambda 3889$ $\AA$ photons. In addition, Clegg (1987) showed that this line is highly sensitive to collisional excitation. The only other HeI line observed in our spectra is the line at $\lambda 3889 \AA$ itself, though it is blended with HI(8). However, as seen from Table 7, once the model strength for $\mathrm{HI}(8)$ is subtracted from the blend, we are left with a tight correlation between this and the model prediction for the line.

The He abundances resulting from our models are seen from Table 8 and Figures $12 a$ and $12 b$ to be exceedingly good, both in terms of a scatter of only $\sim 0.05 \mathrm{dex}$, and in terms of agreement with other published estimates.

\section{b) Nitrogen}

The only nitrogen line that could be consistently measured was [NII] $\lambda 6584$ $\AA$, and although strong, it was sometimes blended somewhat with $\mathrm{H}_{\alpha}$. For a fixed $\bar{Q}$, the $\mathrm{N}$ line strengths scale directly with $\mathrm{N}$ abundance. Thus, after determination of the ionization parameter, adjustment of the $\mathrm{N}$ abundance enabled us to achieve close fits to [NII] $\lambda 6584 \AA$ with our models, and when resolution allowed, to 
[NI] $\lambda 6548 \AA$. In the single case (N138A) where we were able to measure the line

$[\mathrm{NII}] \lambda 5755 \AA$, our model was again successful in predicting the line-strength which confirms that the electron temperature is correctly modelled.

The nitrogen abundances we derived are slightly on the high side of the mean of the values quoted in the literature, as can be seen best in Figures $12 a$ and $12 b$. The reason for this is probably that the single slit position, as used in most previous investigations, tends to underestimate the lower ionization species (like [NI]) with respect to the higher ones, and hence underestimate abundances based only on such lines.

\section{c) Oxygen}

The oxygen abundance is critical to our understanding of the nebulae, and indeed the Magellanic Clouds themselves. It is the most abundant element after hydrogen and helium, and is the principal cooling agent in most nebulae. We went to some lengths to ensure that not only were the total oxygen abundances correct, but the relative abundances of each ionic species were correct. Our chief concern was that the physical parameters and oxygen abundances we chose to represent our models were to a large extent unique. This was tested in several cases by attempting to achieve acceptable results starting from widely different initial conditions. We concluded that the small range of the physical parameters $T_{\text {ion }}$ and $\bar{Q}$ that will produce similar results, would result in a variation in oxygen abundance of between 0.04 and 0.07 dex, although this may be somewhat variable depending on the value of $\bar{Q}$.

Returning to Table 7 we see that for each ionization stage of oxygen there is at least one other line commonly measured in our spectra, but not modelled directly. The close agreement between our models and these observed lines indicates that the observational error is not significant. One indirect result that supports the validity of our models, is the constancy of our derived helium abundances. The modelled $\mathrm{He}$ 
line-strengths are quite sensitive to the value of $T_{\text {ion }}$, due to the effect of helium line blanketing in the exciting star. As a result, the small scatter in He abundance coupled with the close correspondence between our model He line strengths and the observed ones, argues favourably for an accurate physical model. In addition to this, we had the advantage over most other studies in the literature, in that we observed the integrated light from each object, and hence avoided their bias towards the higher ionization levels. On this basis we are confident that we present here a significant improvement in quality in the determination of the oxygen abundances in the Magellanic Clouds, as compared with most previous attempts.

As can be seen from Table 8 and Figures $12 a$ and $12 b$, our average oxygen abundances are in overall agreement with previous estimates. In particular, we see that in the case of the SMC we found a very small scatter of only 0.1dex, thus substantiating the claim that oxygen is well mixed in the SMC. In the LMC, however, we find a significant variation from object to object, that appears to be beyond the bounds of experimental error. This is not inconsistent with the sense of the slight abundance gradient found by Pagel et al. (1978).

\section{d) Neon}

The neon abundance we derive for the SMC is somewhat higher than the results from the literature would indicate, and our results have nearly $0.2 \mathrm{dex}$ of scatter. If we look at the Table 7, we see that these abundances are derived only from [NeIII]. The line at $3967 \AA$, having been determined from the blend at that wavelength by subtraction of the model $\mathrm{HI}(\varepsilon)$ strength, is in good agreement with our models (except in the case of $\mathrm{N} 84 \mathrm{C}$ as discussed previously). Although this ionization stage, [NeII], is the most abundant species for $\mathrm{Ne}$, it is highly sensitive to the absorption edges in the stellar atmospheres employed. Indeed, the [NeII] lines in N127A were totally impossible to model correctly using the assumption of one central exciting star. More realistic models involving mass distributions of stars 
in the central $O B$ associations would presumably alleviate these problems and reduce the scatter in derived $\mathrm{Ne}$ abundances. If, as a result of this problem, we have increased the $\mathrm{Ne}$ abundances artificially too high in our efforts to model the observed line strengths, we would expect to observe a corresponding decrease in the temperature derived from the model ratio of the auroral to nebular [OII] lines. This is due to the $\mathrm{Ne}^{++}$and $\mathrm{O}^{++}$zones being very nearly coextensive. From Table 7 we see that this has not happened, and if anything, the [OII] $\lambda 4363 \AA$ line has been slightly over-estimated by our models (possibly due to an inaccurate estimation of the carbon abundance).

The scatter in Ne abundances for our LMC objects is less extensive, and the average abundance close to that derived from the literature. However, compared to our oxygen abundances, we see from Figures $12 a$ and $12 b$ that our neon abundances are somewhat less deficient. Since both neon and oxygen are thought to be derived from essentially the same high mass stars, this apparent underdeficiency of neon, indicates oxygen may have been depleted to some extent by grain formation.

\section{e) Magnesium}

In addition to the lines given in Table 8, the intercombination line of magnesium, $\mathrm{Mg}] \lambda 4571$, was modelled by the code, and as mentioned in $\S \Pi \mathrm{c}$, it was actually measured in N12A (see Fig. 5). This will allow us to estimate, for this object, the apparent magnesium abundance, and hence the degree of depletion from the mean abundance measured in supergiants in Paper I. However, the code did not include the $\mathrm{Mg}\left(\mathrm{H}^{+}, \mathrm{H}\right) \mathrm{Mg}^{+}$charge-transfer reaction rate calculated by Allan et al . (1988) and, as such, could not accurately compute the magnesium abundance. The abundance that results when the rate coefficient is included will be reported in a future paper. 


\section{f) Sulphur}

The sulphur abundances have been determined in our models from the lines [SII] $\lambda \lambda 6716,6731 \AA$. As can be seen from Table 7, these lines have been closely matched by our models, whereas the model [SIII] lines are systematically overestimated. This overestimation of the [SIII] lines is the same for both the auroral line at $6312 \AA$, and the nebula lines at $9069 \AA$ and $9532 \AA$, so the problem is unlikely to be due to inaccuracies in the collision strengths. It therefore seems likely that the problem lies in an overestimated charge exchange rate for the ion. We investigated this further by turning the charge exchange off for one of our models, and the results were significantly improved, though some overestimate was still present.

The average sulphur abundance we derived for the $\mathrm{LMC}$ is much the same as that estimated by other authors from the literature, but our average abundance for , the SMC is somewhat higher than most previous estimates, while our scatter is very small. However, until the disparity between the [SII] and [SIII] lines is remedied, we are hesitant about making any firm conclusions from our results.

\section{g) Chlorine}

We were fortunate in being able to obtain measures for two lines of chlorine in each of two nebulae in the LMC. The scatter illustrated in Figure $12 a$ is not very meaningful with only two estimates, but when combined with the values from the literature, we would conclude that in the LMC the chlorine deficiency is not significantly different to that of iron.

$$
\text { h) Argon }
$$

We were consistently able to measure one line of [ArIII] and in most cases two lines in each of our objects. Significantly, in one case (N12A), excited by a high temperature central star, we were able to measure in addition, two lines of 
[ArIV], both of which agree well with our model even though they were not specifically modelled. In both the Clouds we see from Figures $12 a$ and $12 b$ that the argon abundances are close to the deficiency of iron, or perhaps a little less deficient, at least in the case of the SMC for which we have very little scatter. In both cases our results are in close agreement with the literature.

\section{THE DETAILED MODELING OF THE SUPERNOVA REMNANTS.}

\section{a) The optical spectra}

The optical spectra of evolved supernova remnants result from radiative shocks moving into material which was part of the original ISM from which the supernova precursor star was formed. We can be confident that this is true for two basic reasons. Firstly, the supernova ejecta is reheated by a reverse shock when the material has interacted with roughly its own mass of interstellar material. It is the thermal pressure from this high temperature gas that drives radiative shocks into the interstellar medium, while the ejecta itself is emitting in the X-ray waveband. Secondly, radiative shocks can only occur where the cooling and recombination timescales are shorter than the evolution timescale of the nebula. The implication of this is that only clouds sufficiently dense to have avoided significant contamination from pre-supernova winds, will have radiative shocks driven into them. The spectra will, however, remain unrepresentative of the ISM chemical abundances if grains can survive both the the supernova event itself, and shock that follows. As far as our present understanding goes, all icy mantle grains would be destroyed by sublimation to large distances from a supernova (see Draine and Salpeter 1979), essentially all remaining volatile mantles would be destroyed by shocks in excess of $50 \mathrm{~km} \mathrm{~s}^{-1}$, the heavier grains (iron and silicates) would be largely destroyed by 
shocks in excess of $70-100 \mathrm{~km} \mathrm{~s}^{-1}$, while the lighter grains (graphite) require shocks in excess of $90-120 \mathrm{~km} \mathrm{~s}^{-1}$ to be significantly depleted (depending critically on the typical radius of the graphite grains). These results are dependant upon the magnetic field to some extent, since as the field compresses the grain gyromotion increases, thus improving the efficiency of the thermal sputtering in the shock (see Cowie 1978, Shull 1979, and Draine and Salpeter 1979). Nevertheless, under the shock conditions apparent in SNRs of the Magellanic Clouds, we would expect almost total grain destruction. Empirical evidence that this is indeed the case, at least for iron and the silicates, and true also for the high-excitation Galactic Herbig-Haro objects, is presented in §VII.

The main problem with determining abundances from shock excited nebulae, is that neither ionization nor thermal equilibrium is achieved at any point in the postshock region. Thus the effects of metallicity, shock conditions and non-equilibrium conditions on the optical spectra must be derived through detailed modeling. Since the pioneering work of Cox (1972) great advances have been made in the level of sophistication of the models (see for example Dopita 1977, Raymond 1979, Shull and McKee 1979, Butler and Raymond 1980, Seab and Shull 1983, Dopita et al. 1984, Cox and Raymond 1985, Hartigan, Raymond, and Hartmann 1987). The MAPPINGS code takes full account of the major advances and uses the most up-todate physical data available.

The effects of "unseen" coolants from lines in the UV ([C], CII], CII, MgII, SiIII etc.) and lines in the infrared ([CII], [OI], [NeII] etc.) are small in the zone where the optical forbidden lines are emitted, and contribute little to the overall cooling rate. Therefore, to a very good approximation, there is a good correlation between the strengths of the forbidden lines with respect to hydrogen and the corresponding elemental abundances.

The physical parameters essential for describing a shock excited gas are: the preshock number density of atoms plus ions, $N_{O}$; the temperature of the gas 
immediately behind the shock, $T_{2}$; the magnetic field, $B$; and the elemental abundances of the gas. The effects of a non-negligible magnetic field are hard to distinguish from an increase in density, so in our models $B$ has been held constant at $0.1 \mu \mathrm{G}$. A degree of support for our taking $B$ to be small comes from the models of Raymond (1979) for instance, which indicate that a large value of $B$ would tend to depress the $[\mathrm{OI}] \lambda 6300$ line strength. In fact the observed strengths for this line were systematically higher than predicted by our final models where $B$ was assumed negligible, so a strong field would only serve to make matters worse.

The temperature $T_{2}$ is determined by the shock velocity $V_{S}$, so in what follows we refer mostly to $V_{S}$ since this is the observable quantity. In Table 9, we show the various estimates for the values of $N_{O}$ and $V_{S}$ derived from the literature. Since it is very unclear exactly which of these best represents the mean shock velocity, we cannot use the results from this table to tie down the shock velocity for each SNR from direct observation. The workers represented in Table 9, are however, in substantial agreement over one thing, the velocities are greater than $\sim 100 \mathrm{~km} \mathrm{~s}^{-1}$, thus the shocks are all moving into regions of almost totally preionized gas.

In our detailed modeling of the supernova remnants, we maintained a constant shock velocity of $150 \mathrm{~km} \mathrm{~s}^{-1}$ for all objects, thus taking full advantage of the above results and the following two observations from Dopita et al. (1984):

1. There is a strong correlation between various line ratios measured from the spectra of a wide variety of SNRs which should not occur if shock velocities are important in determining the optical line emission. The observed trends are taken to be abundance effects with the shock conditions having only a secondary effect on the spectra.

2. The models of Dopita et al. (1984) show that for shock velocities high enough to provide significant pre-ionization of hydrogen, the emergent spectrum depends very little on the precise details of the preionization. This stability against 


\section{TABLE 9}

Shock Velocities and Preshock Densities from the Literature

\begin{tabular}{|c|c|c|c|c|c|}
\hline Object & $\underset{\left(\mathrm{kms}^{-1}\right)}{\mathrm{V}_{\mathrm{s}}}$ & $\begin{array}{l}\mathrm{N}_{0} \\
\left(\mathrm{~cm}^{-3}\right)\end{array}$ & Method for Determining $V_{S}$ & Reference & \\
\hline $\mathrm{H1}$ & $\begin{array}{l}70-150 \\
350 \\
200 \\
90\end{array}$ & $\begin{array}{l}\cdots \\
\cdots \\
300\end{array}$ & $\begin{array}{l}\text { Models of several emission line profiles } \\
\text { Model of spatially resolved H } \beta \text { profile } \\
\text { Models of optical spectra } \\
\text { Half max. velocity dispersion + models }\end{array}$ & $\begin{array}{l}\text { Hartigan } \\
\text { Choe, Bohm and Solf } \\
\text { Hartmann and Raymond } \\
\text { Dopita }\end{array}$ & $\begin{array}{r}1987 \\
1985 \\
1984 \\
1978\end{array}$ \\
\hline $\begin{array}{l}\mathrm{HH} 32 \\
\mathrm{~A}, \mathrm{~B}\end{array}$ & $\begin{array}{l}350 \\
300 \\
150\end{array}$ & $\begin{array}{l}\cdots \\
50 \\
40\end{array}$ & $\begin{array}{l}\text { Models of several emission line profiles } \\
\text { Model of spatially resolved H } \alpha \text { profile } \\
\text { Half max. velocity dispersion + models }\end{array}$ & $\begin{array}{l}\text { Hartigan } \\
\text { Solf, Bobm and Raga } \\
\text { Dopita }\end{array}$ & $\begin{array}{l}1987 \\
1986 \\
1978\end{array}$ \\
\hline HH56 & $65-73$ & 80 & Models of optical spectra & Schwartz and Dopita & 1980 \\
\hline $\begin{array}{l}\mathrm{HH} 101 \\
\mathrm{~N}, \mathrm{~S}\end{array}$ & $\begin{array}{c}170 \\
80\end{array}$ & 获 & $\begin{array}{l}\text { Models of several emission line profiles } \\
\text { Half max. velocity dispersion + models }\end{array}$ & & $\begin{array}{l}1987 \\
1978\end{array}$ \\
\hline $\begin{array}{l}\text { N49 } \\
\text { (LMC) } 8\end{array}$ & $\begin{array}{c}260 \\
80-130 \\
264 \\
200 \\
100\end{array}$ & $\begin{array}{c}\cdots \\
\cdots \\
9-15 \\
67.5\end{array}$ & $\begin{array}{l}\text { Full width at half maximum of } \mathrm{H} \alpha \text { line } \\
\text { Half width at half maximum of } \mathrm{H} \alpha \text { line } \\
\text { Full width at half maximum of } \mathrm{H} \alpha \text { line } \\
\text { Half max velocity dispersion in [OI] } \\
\text { Fully preionized models of optical spectra }\end{array}$ & $\begin{array}{l}\text { Chu and Kennicutt } \\
\text { Shull } \\
\text { Lasker } \\
\text { Dopita } \\
\text { Dopita }\end{array}$ & $\begin{array}{l}1988 \\
1983 b \\
1981 \\
1979 \\
1976\end{array}$ \\
\hline $\begin{array}{l}\text { N63A } \\
(\mathrm{LMC})\end{array}$ & $\begin{array}{c}52 \\
>300 \\
204 \\
110 \\
126 \\
110\end{array}$ & $\begin{array}{c}\ldots \\
300 \\
\ldots \\
20-100 \\
\ldots \\
52-100\end{array}$ & $\begin{array}{l}\text { Full width at half maximum of } \mathrm{H} \alpha \text { line } \\
\text { Models of optical spectra } \\
\text { Improvement over Dopita (1979) results } \\
\text { Half width at half maximum of } H \alpha \text { line } \\
\text { Full width at half maximum of } H \alpha \text { line } \\
\text { Half max velocity dispersion in [OI] }\end{array}$ & $\begin{array}{l}\text { Chu and Kennicutt } \\
\text { Contini } \\
\text { Rosado } \\
\text { Shull } \\
\text { Lasker } \\
\text { Dopita }\end{array}$ & $\begin{array}{l}1988 \\
1987 \\
1986 \\
1983 \mathrm{a} \\
1981 \\
1979\end{array}$ \\
\hline $\begin{array}{l}\text { N103b } \\
\text { (LMC) }\end{array}$ & $\begin{array}{c}34 \\
105\end{array}$ & $\dddot{56-230}$ & $\begin{array}{l}\text { Full width at half maximum of } \mathrm{H} \alpha \text { line } \\
\text { Half max velocity dispersion in [OI] }\end{array}$ & $\begin{array}{l}\text { Lasker } \\
\text { Dopita }\end{array}$ & $\begin{array}{l}1981 \\
1979\end{array}$ \\
\hline $\begin{array}{l}\text { N19 } \\
\text { (SMC) }\end{array}$ & $\begin{array}{c}265 \\
100 \\
70\end{array}$ & $\begin{array}{l}\cdots \\
\ldots 8\end{array}$ & $\begin{array}{l}\text { Full width at half maximum of } \mathrm{H} \alpha \text { line } \\
\text { Full width at half maximum of } \mathrm{H} \alpha \text { line } \\
\text { Half max velocity dispersion in [OI] }\end{array}$ & $\begin{array}{l}\text { Chu and Kennicutt } \\
\text { Lasker } \\
\text { Dopita }\end{array}$ & $\begin{array}{l}1988 \\
1981 \\
1979\end{array}$ \\
\hline $\begin{array}{l}\text { SNR } \\
0104-723 \\
\text { (SMC) }\end{array}$ & $3^{106}$ & $\ldots$ & $\begin{array}{l}\text { Relation (Dopita 1979) between mean } \\
\text { optical diameter (Mathewson et al. 1984) } \\
\text { and velocity. }\end{array}$ & $\cdots$ & $\ldots$ \\
\hline
\end{tabular}


shock conditions was shown by Binette, Dopita and Tuohy (1985) to persist up to shock velocities of more than $200 \mathrm{~km} \mathrm{~s}^{-1}$, thus explaining the observed correlations in line ratios for all observed SNRs.

We replotted the data from Dopita et al. (1984) in Figures $13 a$ to $13 d$, and included points derived from our own work as well as further points from the literature published since the paper of Dopita et al. (1984). This introduces a number of new SNRs to the original diagrams, especially at the low abundance end, and the continued correlation further supports the conclusion that abundance is the main factor in determining the optical spectra for these objects. The major ramifications are that all detected SNRs are driven by shocks exceeding $130 \mathrm{~km} \mathrm{~s}^{-1}$, and no other processes, like for instance grain formation or magnetic fields, make a significant contribution to the optical spectra.

In order to check, however, that our data could not be fitted better by shock velocities in the range $100-130 \mathrm{~km} \mathrm{~s}^{-1}$, where variations in preionization on the output spectrum are already small, we ran a series of models at $105 \mathrm{~km} \mathrm{~s}^{-1}$ for all our objects. The results of these were considerably less satisfactory in all cases, thus forcing us to assume the more stable velocity of $150 \mathrm{~km} \mathrm{~s}^{-1}$ (more stable in terms of the variation in line strengths with shock velocity). We would not expect to observe emission from shocks much in excess of $200 \mathrm{~km} \mathrm{~s}^{-1}$ as they would no longer become radiative within the dynamic evolution time scale of the nebula.

The only other possible scenario imaginable would be for an object to have a shock velocity on the rising portion of the [OIM] curve (see fig. 5 of Dopita et al. 1984). In this case the [OIII] lines would cease to be of use as abundance indicators, and instead determine the shock velocity very precisely. Any deviation from a critical velocity (of around $79 \mathrm{~km} \mathrm{~s}^{-1}$ ) by as much as $1 \mathrm{~km} \mathrm{~s}^{-1}$ would result in either no observable [OIII], or close to the fully ionized value. Such a situation is so improbable as to be discounted immediately. 
Figure 13a The distribution SNRs (filled symbols) and Galactic HH-objects (open circles) (from this work, Strom et al. 1974, and the references cited in Table 10) on a diagram showing the fluxes relative to $H_{\alpha}$, of $[N I] \lambda \lambda 6548,6584$ versus [SI] $\lambda 6731$. The filled circles represent the combined results from the Galaxy (Leibowitz and Danziger 1983, and references therein, Binette $e t$ al. 1982, and references therein, and Dopita 1980), M31 (Blair et al. 1981, 1982, and Dennefeld and Kunth 1981) and M33 (Dopita et al. 1980), while the filled triangles and squares represent the LMC (this work, Dopita et al. 1980, and Danziger and Leibowitz 1985) and SMC (this work) respectively.

Figure 13b As for Figure 13a for the fluxes relative to $\mathrm{H} \beta$ of [OIm] $\lambda \lambda 4959,5007$ versus $[\mathrm{OII}] \lambda \lambda 3726,3729$.

Figure 13c As for Figure 13a for $[\mathrm{NI}] \lambda \lambda 6548,6584 / \mathrm{H}_{\alpha}$ versus [OIm] $\lambda \lambda 4959,5007 / \mathrm{H} \beta$.

Figure 13d As for Figure 13a for [OIm] $\lambda \lambda 4959,5007 / \mathrm{H} \beta$ versus [Sm] $\lambda 6731 / \mathrm{H}_{\alpha}$. 

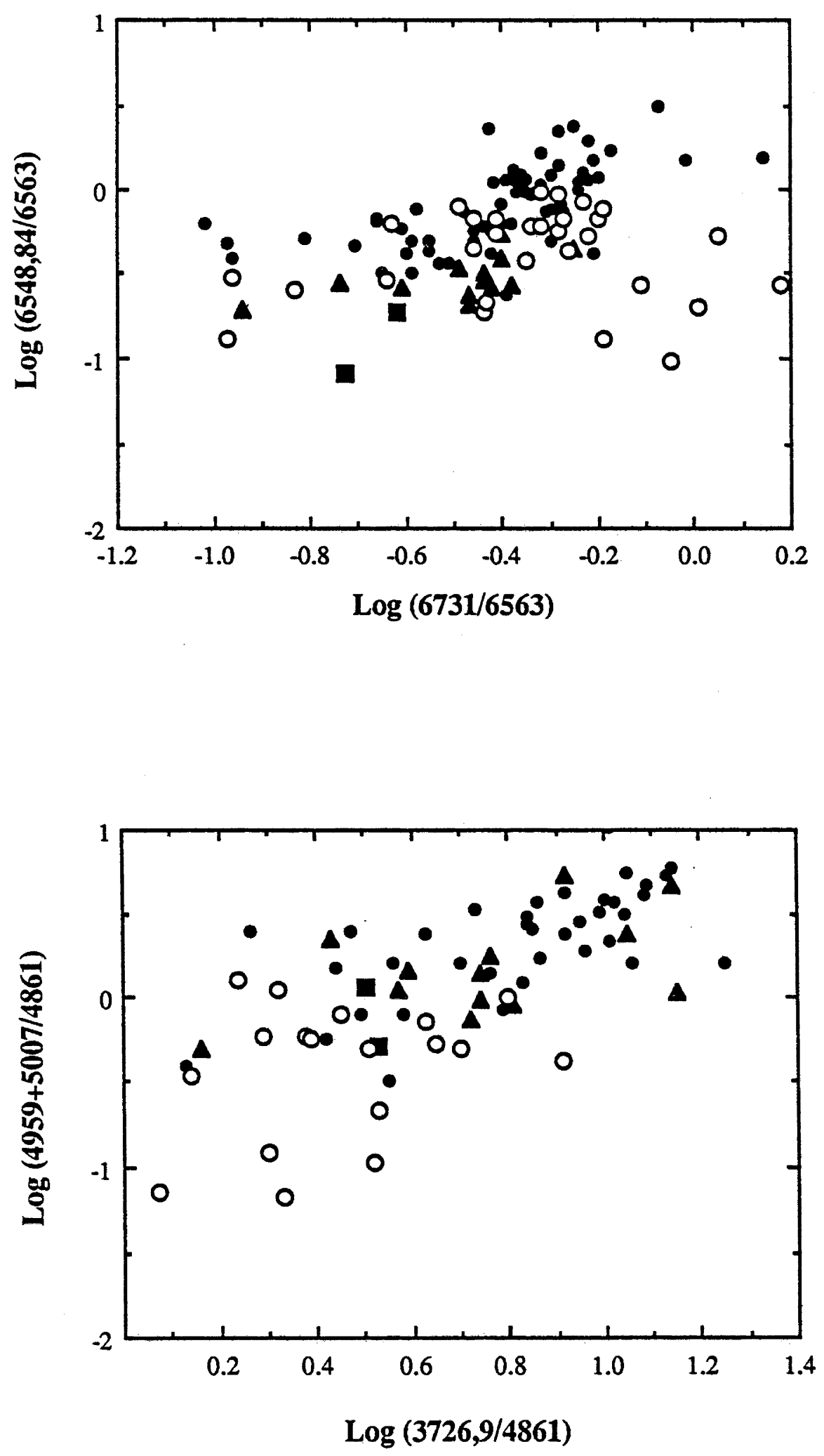

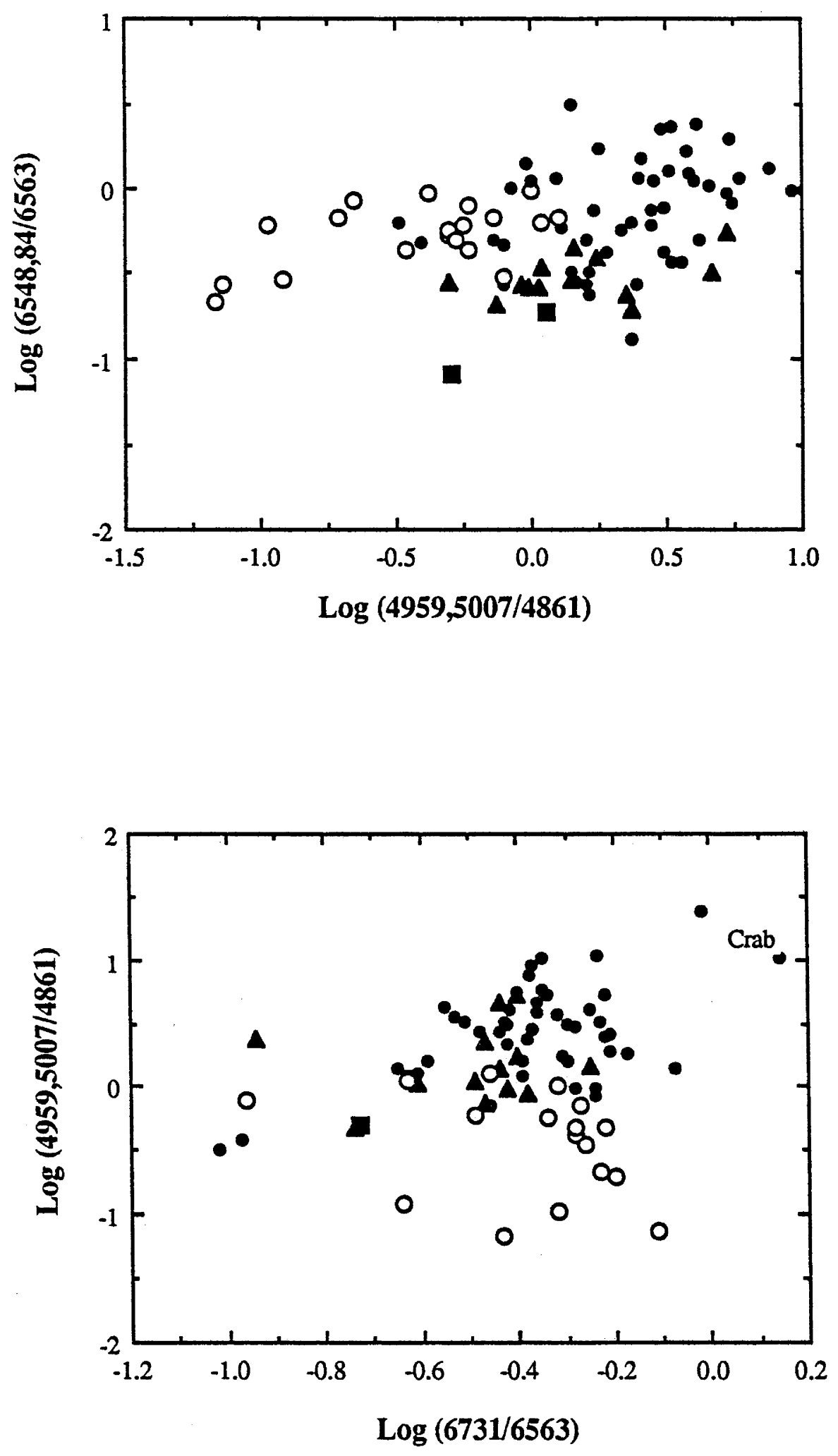
Once the shock velocity was fixed, we were left with only the densities and the detailed abundances as free parameters. According to Dopita (1979), the preshock density $\mathrm{N}_{\mathrm{O}}$ is related to the density in the recombination region $\mathrm{N}_{[\mathrm{Sm}]}$ (as determined from $[S I I] \lambda 6716 / \lambda 6731$ ratio), and the shock velocity $V_{100}$ (in units of $100 \mathrm{~km} \mathrm{~s}^{-1}$ ), though the equation

$$
\mathrm{N}_{[\mathrm{SII}]}=45\left(\mathrm{~V}_{100}\right)^{2} \mathrm{~N}_{\mathrm{o}}
$$

equation 7.

Hence the preshock density is correctly determined for a given shock velocity if our model successfully predicts the [SII] ratio. The only problem occurs in the low density limit where the [SI] ratio loses all sensitivity (as mentioned previously in relation to the $\mathrm{H}$ II regions). This appears to be the case in both the SMC supernova remnants, for which we have simply assumed that $N_{[S I]}=10 \mathrm{~cm}^{-3}$ for modeling purposes.

The final task in the modeling procedure was to vary the elemental abundances until we obtained the closest possible fit to the observed spectra. This involved making an initial guess at the oxygen abundances using the diagnostic diagrams of Dopita et al. (1984) (their figures 8 and 10), then refining our models progressively using MAPPINGS until the best fit was achieved.

The results are shown in Table 10A, where we compare the model line strengths $\log I_{m}$, with the observed line strengths $\log I_{o}$. We include in this table observations and a model fit to the Cygnus Loop, a much studied and mature Galactic SNR. As in Table 7, those lines for which a match was demanded are shown in italics, and the model strength of 10.51 for $\mathrm{HI}(8)$, was subtracted from the blend at $3888 \AA$ in order to estimate the strength of the HeI line at $3889 \AA$. The [SIII] line at $9532 \AA$ was deblended by subtracting the theoretical strength for $\mathrm{HI}(\mathrm{P} 8)$ of 3.6.

For reasons that are at present unclear to us, the fit to the He lines is relatively poor, certainly when compared with the results derived from the $\mathrm{H}$ II regions (see 


\section{TABLE 10A}

Optical and Near-infrared

Model Line Strengths for the Supernova Remnants

\begin{tabular}{|c|c|c|c|c|c|c|c|c|c|c|c|c|c|}
\hline \multirow{3}{*}{$\begin{array}{l}\text { Ion } \\
\text { HI }\end{array}$} & \multirow{3}{*}{$\begin{array}{c}\begin{array}{c}\text { Lamda } \\
(\AA)\end{array} \\
3970.7\end{array}$} & \multicolumn{4}{|c|}{ SMC } & \multicolumn{6}{|c|}{ LMC } & \multirow{2}{*}{\multicolumn{2}{|c|}{ 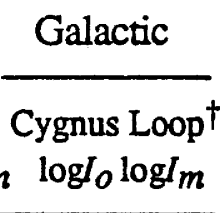 }} \\
\hline & & \multicolumn{2}{|c|}{$\begin{array}{c}N 19 \\
\log I_{o} \log I_{m}\end{array}$} & \multicolumn{2}{|c|}{$\begin{array}{c}\text { SNR0104-723 } \\
\log I_{o} \log I_{m}\end{array}$} & \multicolumn{2}{|c|}{$\begin{array}{c}\mathrm{N} 49 \\
\log I_{o} \log I_{m}\end{array}$} & \multicolumn{2}{|c|}{$\begin{array}{c}\text { N63A } \\
\log I_{o} \log I_{m}\end{array}$} & \multicolumn{2}{|c|}{$\begin{array}{l}\mathrm{N} 103 \mathrm{~b} \\
\log I_{o} \log I_{m}\end{array}$} & & \\
\hline & & $\cdots$ & 1.19 & $\cdots$ & 1.19 & $\ldots$ & 1.19 & 0.49 & 1.19 & $\cdots$ & 1.20 & $\ldots$ & $\cdots$ \\
\hline & 4101.7 & 1.45 & 1.41 & 1.42 & 1.41 & 1.44 & 1.41 & 1.43 & 1.41 & 1.42 & 1.41 & $\ldots$ & $\cdots$ \\
\hline & 4340.5 & 1.42 & 1.67 & 1.65 & 1.67 & 1.67 & 1.67 & 1.64 & 1.67 & 1.68 & 1.67 & 1.65 & 1.66 \\
\hline & 4861.3 & 2.00 & 2.00 & 2.00 & 2.00 & 2.00 & 2.00 & 2.00 & 2.00 & 2.00 & 2.00 & 2.00 & 2.00 \\
\hline & 6562.8 & 2.60 & 2.46 & 2.63 & 2.47 & 2.64 & 2.47 & 2.64 & 2.46 & 2.48 & 2.46 & 2.53 & 2.48 \\
\hline $\mathrm{HeI}$ & 3888.7 & $\ldots$ & 0.58 & 1.26 & 0.57 & 1.39 & 0.49 & 1.27 & 0.51 & 1.35 & 0.56 & $\ldots$ & \\
\hline & 5875.7 & $\ldots$ & 0.68 & 0.88 & 0.67 & 1.10 & 0.61 & 1.04 & 0.61 & 1.05 & 0.66 & 1.04 & 1.04 \\
\hline & 6678.2 & 0.67 & 0.14 & $\ldots$ & 0.12 & 0.80 & 0.06 & 0.69 & 0.06 & $\ldots$ & 0.11 & ... & $\cdots$ \\
\hline & 7065.2 & $\ldots$ & 0.75 & $\ldots$ & -0.26 & 0.63 & -0.37 & 0.58 & -0.33 & 0.54 & -0.28 & $\ldots$ & $\ldots$ \\
\hline HeII & 4685.7 & 0.71 & 0.61 & 0.57 & 0.61 & 0.75 & 0.43 & 0.32 & 0.50 & 0.49 & 0.55 & $\ldots$ & $\ldots$ \\
\hline [N] & 5199. & 0.70 & 1.40 & 0.08 & 0.93 & 0.60 & 1.03 & 0.79 & 1.48 & 0.23 & 1.00 & $\ldots$ & $\ldots$ \\
\hline [NII] & 5754.6 & ... & 0.27 & $\ldots$ & -0.20 & 0.08 & 0.23 & $\ldots$ & 0.59 & $\ldots$ & 0.39 & 0.78 & 1.11 \\
\hline & 6548.1 & ... & 1.27 & 1.21 & 0.80 & $\ldots$ & 1.36 & $\ldots$ & 1.56 & 1.43 & 1.28 & 1.98 & 1.98 \\
\hline & 6583.5 & 1.76 & 1.74 & 1.27 & 1.27 & 2.11 & 1.83 & 2.05 & 2.03 & 1.76 & 1.75 & 2.45 & 2.45 \\
\hline [OI] & 6300.3 & 1.71 & 1.54 & 1.81 & 1.59 & 2.10 & 1.80 & 2.08 & 1.74 & 2.04 & 1.86 & 1.71 & 1.61 \\
\hline & 6363.8 & 1.34 & 1.05 & 1.40 & 1.11 & 1.65 & 1.32 & 1.59 & 1.26 & 1.58 & 1.37 & 1.20 & 1.15 \\
\hline [OI] & 3727.5 & 2.50 & 2.50 & 2.53 & 2.55 & 2.76 & 2.73 & 2.57 & 2.54 & 2.16 & 2.18 & 3.08 & 3.11 \\
\hline & 7325 & 0.76 & 1.15 & $\ldots$ & 1.21 & 1.56 & 1.58 & 1.39 & 1.49 & $1.61:$ & $: 1.64$ & 1.76 & 51.86 \\
\hline [OM] & 4363.2 & $\ldots$ & 0.95 & 0.40 & 1.00 & $\ldots$ & 1.06 & $\ldots$ & 1.03 & $\ldots$ & 0.99 & 1.38 & 31.28 \\
\hline & 4958.9 & 1.31 & 1.45 & 1.06 & 1.50 & 1.64 & 1.58 & 1.44 & 1.53 & 1.03 & 1.49 & 2.04 & 41.93 \\
\hline & 5006.9 & 1.97 & 1.91 & 1.59 & 1.95 & 2.11 & 2.04 & 1.91 & 1.99 & 1.58 & 1.95 & 2.53 & 32.40 \\
\hline [NeIII] & 3868.8 & 1.22 & 0.99 & 0.76 & 0.77 & 1.56 & 1.53 & 1.07 & 1.01 & 1.32 & 1.30 & 1.88 & 81.88 \\
\hline$[\mathrm{Sm}]$ & 4072.5 & 1.03 & 1.00 & 0.79 & 0.94 & 1.45 & 1.58 & 1.46 & 1.46 & 1.59 & 1.51 & 1.28 & 1.36 \\
\hline & 6716.4 & 2.17 & 2.14 & 2.05 & 2.08 & 2.13 & 2.15 & 2.04 & 2.01 & 1.49 & 1.50 & 2.23 & 2.18 \\
\hline & 6730.8 & 1.98 & 1.98 & 1.90 & 1.91 & 2.24 & 2.30 & 2.15 & 2.14 & 1.73 & 1.72 & 2.15 & 2.17 \\
\hline & 10330 & 0.81 & 0.61 & $\ldots$ & 0.55 & 0.81 & 1.19 & 1.00 & 1.08 & $\ldots$ & 1.12 & $\ldots$ & ... \\
\hline [SII] & 9532.2 & 1.01 & 1.18 & $\ldots$ & 1.14 & 1.35 & 1.52 & 1.51 & 1.30 & 0.85 & 1.05 & $\ldots$ & ... \\
\hline [ArII] & 7135.8 & $\ldots$ & 0.26 & ... & 0.26 & 0.80 & 0.78 & 0.83 & 0.88 & 0.94 & 0.93 & $\ldots$ & $\ldots$ \\
\hline & 7751.1 & ... & -0.36 & $\ldots$ & -0.36 & 0.15 & 0.17 & 0.28 & 0.26 & $\ldots$ & 0.31 & $\ldots$ & $\ldots$ \\
\hline
\end{tabular}

$\dagger$ Modelled with a shock velocity of $122 \mathrm{kms}^{-1}$ and a magnetic field of $0.5 \mu \mathrm{g}$. 


\section{TABLE 10B}

UV

Model Line Strengths for the Supernova Remnants

\begin{tabular}{|c|c|c|c|c|c|}
\hline \multirow{3}{*}{ Ion } & \multirow{3}{*}{$\begin{array}{l}\text { Lamda } \\
(\AA)\end{array}$} & \multicolumn{2}{|c|}{ Galaxy } & \multicolumn{2}{|c|}{ LMC } \\
\hline & & \multicolumn{2}{|c|}{ Cygnus Loop ${ }^{\dagger}$} & \multicolumn{2}{|c|}{$\mathrm{N} 49$} \\
\hline & & $\log J_{O}$ & $\log I_{m}$ & $\log I_{0}$ & $\log I_{m}$ \\
\hline HeII & 1640 & 1.54 & $\ldots$ & 1.59 & $\ldots$ \\
\hline CII & 1335 & 1.20 & 2.36 & 1.53 & 1.76 \\
\hline CIII & 1906 & 2.29 & 2.26 & 1.89 & 1.73 \\
\hline CIV & 1549 & 1.93 & 2.17 & 1.95 & 1.87 \\
\hline $\mathrm{Nm}]$ & 1748 & 1.53 & 1.36 & 1.52 & 0.66 \\
\hline NIV] & 1486 & 1.20 & 1.52 & 1.15 & 1.27 \\
\hline NV & 1240 & $1.40^{*}$ & 0.70 & 1.32 & 1.89 \\
\hline [OM] & 2470 & 1.82 & 1.74 & $\ldots$ & 1.36 \\
\hline OII] & 1664 & 1.80 & 2.12 & 1.72 & 2.05 \\
\hline Sill & 1822 & 1.34 & $\ldots$ & 1.23 & $\ldots$ \\
\hline Sill] & 1886 & 1.75 & 1.75 & 1.72 & 1.74 \\
\hline SIV] & $1395)$ & & & & \\
\hline SiIV & ) & 1.63 & 1.87 & 1.99 & 2.21 \\
\hline OIV] & 1403) & & & & \\
\hline $\mathrm{CII}$ & ) & & & & \\
\hline SiII & ) & 2.43 & 2.13 & $\ldots$ & 1.78 \\
\hline [OMI] & ) & & & & \\
\hline
\end{tabular}

* Approximate de-blend

$\dagger$ Modelled with a shock velocity of $122 \mathrm{kms}^{-1}$ and a magnetic field of $0.5 \mu \mathrm{g}$. 
Table 7). As a result, we have not made use of these results to derive any further information on the He abundance in the Clouds. The [OI] line strengths are predicted somewhat low by our models (a factor of $\sim 1.8$ ). This may be a result of a somewhat extended zone of material maintained at a temperature of 10,000 to $20,000 \mathrm{~K}$ by reflection, refraction and collision of the primary shocks with density inhomogeneities in the ISM (as first suggested by Dopita 1978). As a result of this, there would be a large amount of collisional excitation but little additional ionization, thus strengthening the [OI] lines and increasing the $\mathrm{H}_{\alpha} / \mathrm{H} \beta$ ratio. Alternatively, the underestimation of the $[\mathrm{O}]$ line strengths may be due to an inadequate knowledge of the physical constants for the transitions. Fortunately, these lines are not critical in determining the oxygen abundance, so the problem was not pursued further in this work.

Except for nitrogen, most other lines are quite well predicted by our models. We have fitted [NII] $\lambda 6584$ with our models since we know that this line scales well with abundance in the recombination zone. However, we are then left with [N] $\lambda 5199$ overestimated by a factor of $\sim 6$. Fortunately, we have additional data in the UV for one well observed object in the LMC, N49.

\section{b) The UV spectra}

The UV line-strengths for N49, as well as those for the Cygnus Loop, are given in Table 10B, and are derived from the same IUE spectra as described by Benvenuti, Dopita, and D'Odorico (1980). In this case, however, an alternative technique was used to convert the line fluxes to the scale of $H \beta=100$. A strong $U V$ continuum was observed that results from the collisionally enhanced two-photon continuum of hydrogen. This could be accurately computed with respect to $\mathrm{H} \beta$, thus allowing the direct scaling of each line, rather than employing a rather uncertain estimate of the reddening. 
The UV spectrum of N49 corresponded in position to roughly the same as that studied by Osterbrock and Dufour (1973), it is therefore more correct to model their value for $[N I I] \lambda 6584\left(\log I_{O}=1.89\right)$, rather than our own. On successfully modeling this line, we see that we underestimate $\operatorname{NIm} \lambda 1748$, achieve a reasonable fit to NIV] $\lambda 1486$, and overestimate both [NI] $\lambda 5199$ and NV $\lambda 1240$. Clearly, altering the $\mathrm{N}$ abundance is not going to bring all the model lines into any closer agreement with the observed lines, and decreasing the shock velocity to $105 \mathrm{~km} \mathrm{~s}^{-1}$ has the effect of increasing the average error between model and observation by $\sim 0.15$ dex. Similarly, increasing the shock velocity by $45 \mathrm{~km} \mathrm{~s}^{-1}$ would be expected to increase the average error by an even higher margin. We believe that the [NII] $\lambda 6584$ line is an adequate means to determine the $\mathrm{N}$ abundance, although it is clear that such an abundance has an increased uncertainty. Finally, it is worth mentioning on this subject, that asimilar overestimate to our own for the [NT] lines was made in the models of Shull and McKee (1979), while Butler and Raymond (1980) predicted strengths 8 times smaller. Butler and Raymond mentioned that the difference "could not easily be attributed to differences in assumed abundances, excitation rates, or charge transfer".

Another advantage of having observations in the UV part of the spectrum, is the information it gives us on the otherwise unseen coolants $\mathrm{C}$ and $\mathrm{Si}$. If their abundance is overestimated to a significant degree, then the abundances of several elements, most notably Ne from the single line [NeIIT] 33869 (see Dopita 1977) will be underestimated. This is due to the fixed amount of energy available for radiation in any particular ionization zone. The combined emissions of all the coolants in that zone must be in constant ratio with the hydrogen Balmer lines. If the unseen coolants are responsible for more of that radiation than we estimate, then all other coolants must be responsible for correspondingly smaller proportion of the radiation. 
In Tables 11, and 12 we include the finally derived abundances for all the elements we were able to observe in the supernova remnants. We include in Table 11 the preshock densities we used for our models, although as mentioned previously, the values for the SMC supernova remnants are somewhat arbitrary. In Table 12 we also present the mean abundance results for each Cloud from the other types of object studied in this series of papers. 
TABLE 11

Derived Preshock Densities and Abundances

For the Supernova Remnants

\begin{tabular}{|c|c|c|c|c|c|c|}
\hline \multirow[b]{2}{*}{ Parameter } & \multicolumn{2}{|r|}{ SMC } & \multicolumn{3}{|c|}{ LMC } & \multirow{2}{*}{$\begin{array}{c}\text { Galactic } \\
\text { Cygnus } \\
\text { Loop }\end{array}$} \\
\hline & N19 & SNR0104-723 & N49 & N63A & $\mathrm{N} 103 \mathrm{~b}$ & \\
\hline Preshock density $N_{O}\left(\mathrm{~cm}^{-3}\right)$ & 0.10 & 0.10 & 15.00 & 25.00 & 180.00 & 6.00 \\
\hline $12+\log (\mathrm{C} / \mathrm{H})$ & $\ldots$ & $\ldots$ & 7.66 & $\ldots$ & $\ldots$ & 8.00 \\
\hline $12+\log (\mathrm{N} / \mathrm{H})$ & 7.11 & 6.64 & 7.40 & 7.45 & 7.26 & 8.18 \\
\hline $12+\log (\mathrm{O} / \mathrm{H})$ & 7.90 & 7.95 & 8.54 & 8.10 & 8.11 & 8.84 \\
\hline $12+\log (\mathrm{Ne} / \mathrm{H})$ & 7.00 & 6.78 & 7.95 & 7.11 & 7.45 & 8.11 \\
\hline $12+\log (\mathrm{Si} / \mathrm{H})$ & $\ldots$ & $\ldots$ & 7.48 & $\ldots$ & $\ldots$ & 7.26 \\
\hline $12+\log (\mathrm{S} / \mathrm{H})$ & 6.48 & 6.41 & 7.00 & 6.62 & 6.40 & 7.18 \\
\hline $12+\log (\mathrm{Ar} / \mathrm{H})$ & ... & $\ldots$ & 6.65 & 6.51 & 6.60 & $\ldots$ \\
\hline $12+\log (\mathrm{Ca} / \mathrm{H})$ & $\ldots$ & $\ldots$ & 6.30 & 6.01 & 6.13 & $\ldots$ \\
\hline $12+\log (\mathrm{Cr} / \mathrm{H})$ & $\ldots$ & $\ldots$ & 5.54 & 5.11 & 5.40 & $\ldots$ \\
\hline $12+\log (\mathrm{Fe} / \mathrm{H})$ & 7.06 & 6.92 & 7.30 & 7.12 & 7.25 & $\ldots$ \\
\hline $12+\log (\mathrm{Ni} / \mathrm{H})$ & $\ldots$ & $\ldots$ & 5.97 & 5.73 & 5.92 & $\ldots$ \\
\hline
\end{tabular}




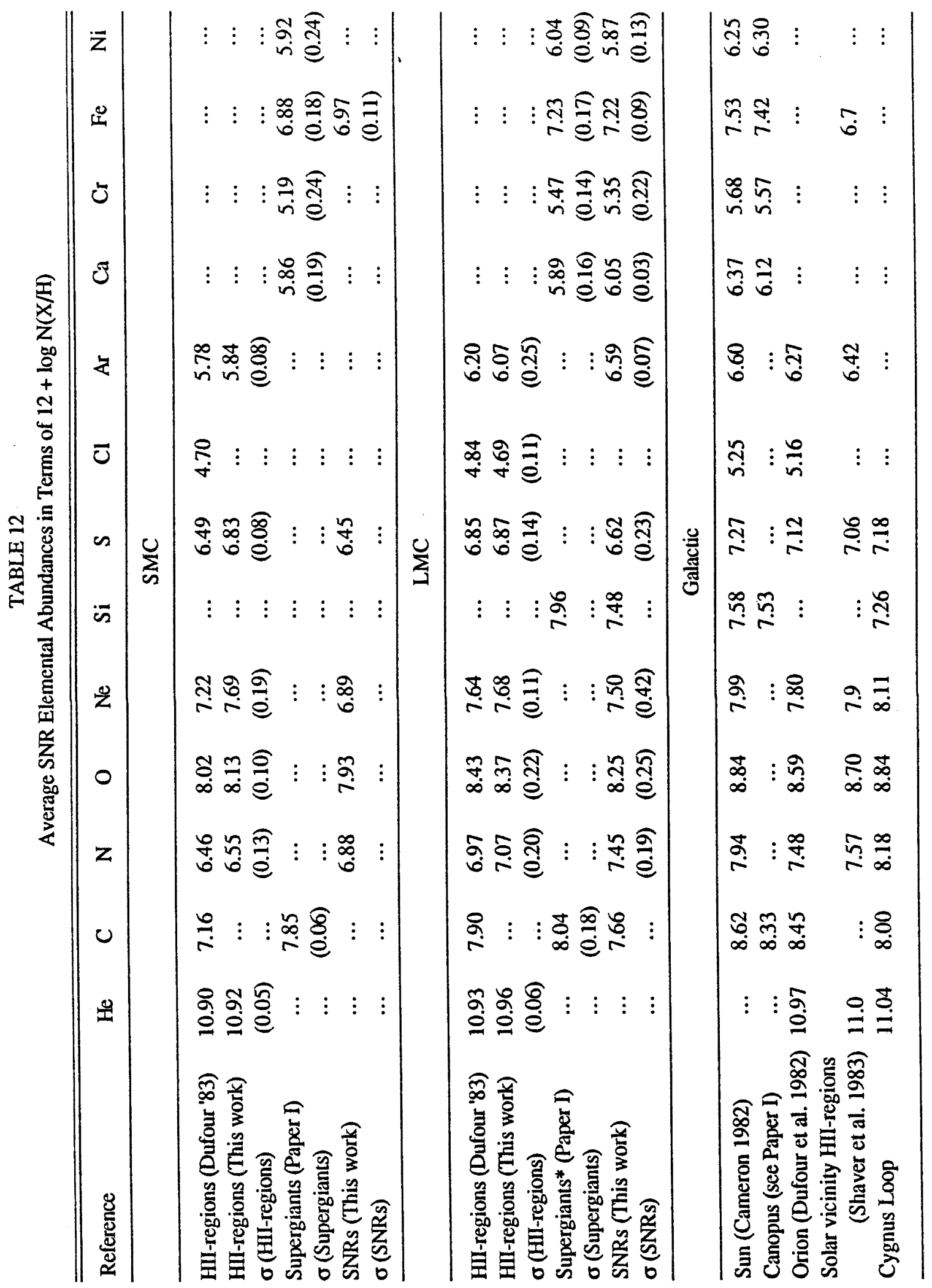




\section{THE HEAVY METALS}

We have included in Figures $13 a$ to $13 d$ data points derived from the spectra of $\mathrm{H}-\mathrm{H}$ objects in our own Galaxy. Clearly these objects are fundamentally different from the SNRs, in that they do not conform to the correlations between line ratios observed for the SNRs. Indeed, from Figures $13 c$ and $13 d$ we see that the two types of object are very nearly entirely separated. Since the densities encountered in $\mathrm{H}-\mathrm{H}$ objects are not substantially different from those found in typical SNRs (see Table 9), the only possible conclusion is that the shock velocities for all the $\mathrm{H}-\mathrm{H}$ objects are lower than $100 \mathrm{~km} \mathrm{~s}^{-1}$, or at least, low velocity shocks dominate the optical spectrum, even if high velocity shocks are also present (as indicated again in Table 9). Hydrogen is therefore not fully preionized in the shocks, and the line ratios depend critically on the shock velocities. In addition, any attempt at modeling these objects with a single shock velocity is unrealistic since under such conditions the geometry of the shocks becomes important, and to date, even the most intricate model falls far short of adequately predicting the observed spectra. For instance, the latest attempt to model the most extensively observed $\mathrm{H}-\mathrm{H}$ object, $\mathrm{HH} 2 \mathrm{~A}$ (see Raymond, Hartigan and Hartmann 1988), predicts an [OII] $\lambda 3727 \AA$ line that is too small by at least a factor of 2 (depending upon the adopted reddening), and an [OII] $\lambda 5007 \AA$ line that is at least $50 \%$ in error.

We have observed the Galactic $\mathrm{H}-\mathrm{H}$ objects because they are clearly shock excited objects, and their proximity to the Sun ensures that their elemental abundances should be close to solar. Evidence confirming this assumption comes from the work of Brugel, Böhm and Mannery (1981). They performed accurate, absolute abundance analyses of the objects $\mathrm{HH} 1(\mathrm{NW}), \mathrm{HH} 2 \mathrm{~A}, \mathrm{HH} 2 \mathrm{G}, \mathrm{HH} 2 \mathrm{H}$ and $\mathrm{HH} 32$ using two-component models, and less accurate analysis of five other $\mathrm{H}-\mathrm{H}$ objects. They concluded that, at least for the elements $\mathrm{N}, \mathrm{O}, \mathrm{Ne}, \mathrm{S}, \mathrm{Ca}$ and $\mathrm{Fe}$, the $\mathrm{H}-\mathrm{H}$ objects have normal ("cosmic") abundances, and reiterate that "in no case is 
there any evidence for metal depletion due to dust formation in an Herbig-Haro object". The only apparent depletion observed by Brugel, Böhm and Mannery in their objects was with CI, which they left still inadequately explained.

Additional evidence that the heavy metal abundances of the $\mathrm{H}-\mathrm{H}$ objects are close to solar abundance comes from the extensive photometry of members of the Orion OB 1 association by Warren and Hesser (1978). From their Strömgren photometry they concluded that a group of F-type stars in the association, have "metal abundances similar to F- and G-type dwarfs in the solar neighborhood". The $\mathrm{HH} 1-\mathrm{HH} 2$ complex is in the same vicinity, so we have confirming indirect evidence that, at least for these objects, the abundances are close to solar.

Since the heavy elements are not major coolants in either $\mathrm{H}-\mathrm{H}$ objects or SNRs, it follows that, to first order, their emission line strengths should be directly proportional to their abundances. If this is so, then a simple comparison between the line strengths from the two types of object should indicate the relative abundances of the heavy metals. However, there are two effects that could systematically affect the line strengths.

The first effect that must be taken out is the enhancement of the line strengths at high densities due to the diversion of radiant energy from coolants affected by collisional de-excitation (such as [SII] and [OII]) into those species which are unaffected (which include [CaIm, [ $\mathrm{CrI}],[\mathrm{FeIM}]$ and [Nim]). This effect may be seen for $\mathrm{H}-\mathrm{H}$ objects in Figure 14 for instance, where we show the correlation between $\log I([\mathrm{Fe}] \lambda 7155 \AA$ ) (on a scale where $\log I(\mathrm{H} \beta)=2.00$ ) and $\log N$ as derived from the [SII] ratio (see Fig. 11). The filled circles represent the classical $\mathrm{H}-\mathrm{H}$ objects with reliable spectra taken from this work and the literature (see Table 13), while the open triangle represents Burnham's Nebula as derived from Solf, Böhm and Raga (1988) which we shall return to a little later.

Using the Galactic data, we corrected the [FeII] line strengths of both the $\mathrm{H}-\mathrm{H}$ objects and Magellanic Cloud SNRs for systematic trends with density. For those 
Figure 14 The correlation between the $[\mathrm{Fe}] \lambda 7155$ line strength and the density derived from the line ratio $[S \Pi] \lambda 6716 / 6731$ (see Fig. 11). 


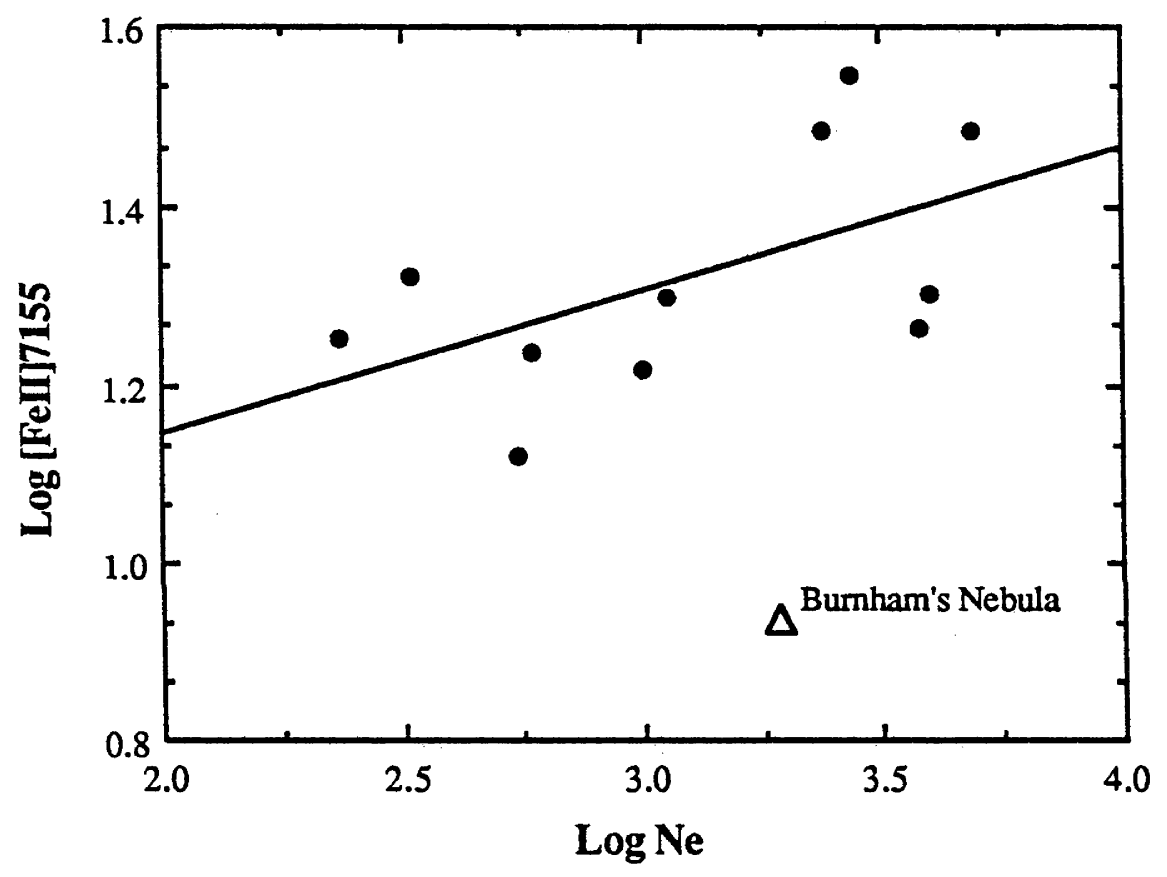




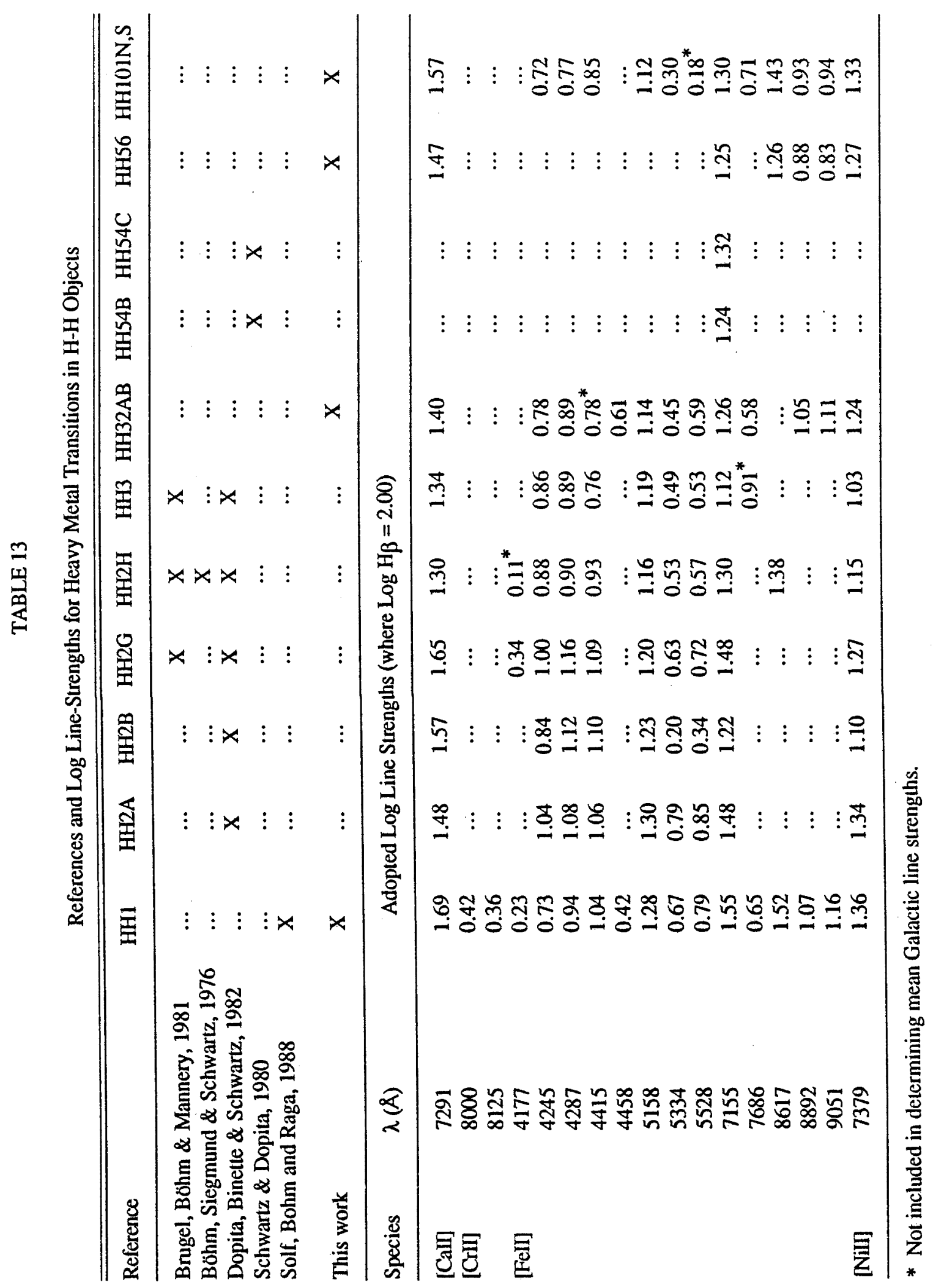


lines of [FeII] having no well defined trend with density (due to lack of data), we estimated the slope of the trend presumed to be present from the measured slopes of the other lines plotted as a function of excitation potential in Figure 15. This same figure was also used to determine the slopes of the trends with density for our analyses of $\mathrm{Cr}$ and $\mathrm{Ca}$ as they were otherwise ill-determined.

The second effect that should be considered is the possible dependance of line strength on the shock velocity. A good diagnostic for the shock velocity is the [OI] $\lambda 6300 \AA$ line, since it tends to increase monotonically with velocity until collisional de-excitation becomes important (see the models of Hartigan, Raymond and Hartmann 1987). However we found little evidence for a correlation with [OI] line strength in the Galactic $\mathrm{H}-\mathrm{H}$ objects, and there was certainly no evidence for one in the Magellanic Cloud SNRs.

As a result, no corrections for shock velocity were attempted, and the results for each individual line, and the average depletions from solar abundance for each object, are presented in Table 14, while the absolute abundances were included in Table 11. We also included our results for Burnham's nebula (using the data from Solf, Böhm and Raga 1988) in Table 14 since we were intrigued at the systematic depletion of the heavy metal lines in this nebula compared to all the H-H objects we studied.Clearly this object (the nebula surrounding $\mathrm{T}$ Tauri) is significantly different from the typical $\mathrm{H}-\mathrm{H}$ objects in that it has $\sim 0.4$ dex depletion in the heavier elements, probably due to a higher survival rate of dust grains in the shock (as suggested by Solf, Böhm and Raga themselves). Significantly, no H-H object that we studied showed any sign of such a systematic depletion, thus indicating that grains are normally wholly destroyed in a shock event. Although some H-H objects are known that do show a depletion, these are usually of low excitation.

From Table 14 it is apparent that the quality of our Fe abundance determinations for the LMC are very good, having errors of only $\pm 0.1 \mathrm{dex}$. The variation in average Fe abundances between the SNRs in our LMC sample, as seen 
Figure 15 The correlation between the Fe/density gradient (slope) and the excitation potential of the upper level of the transition. 


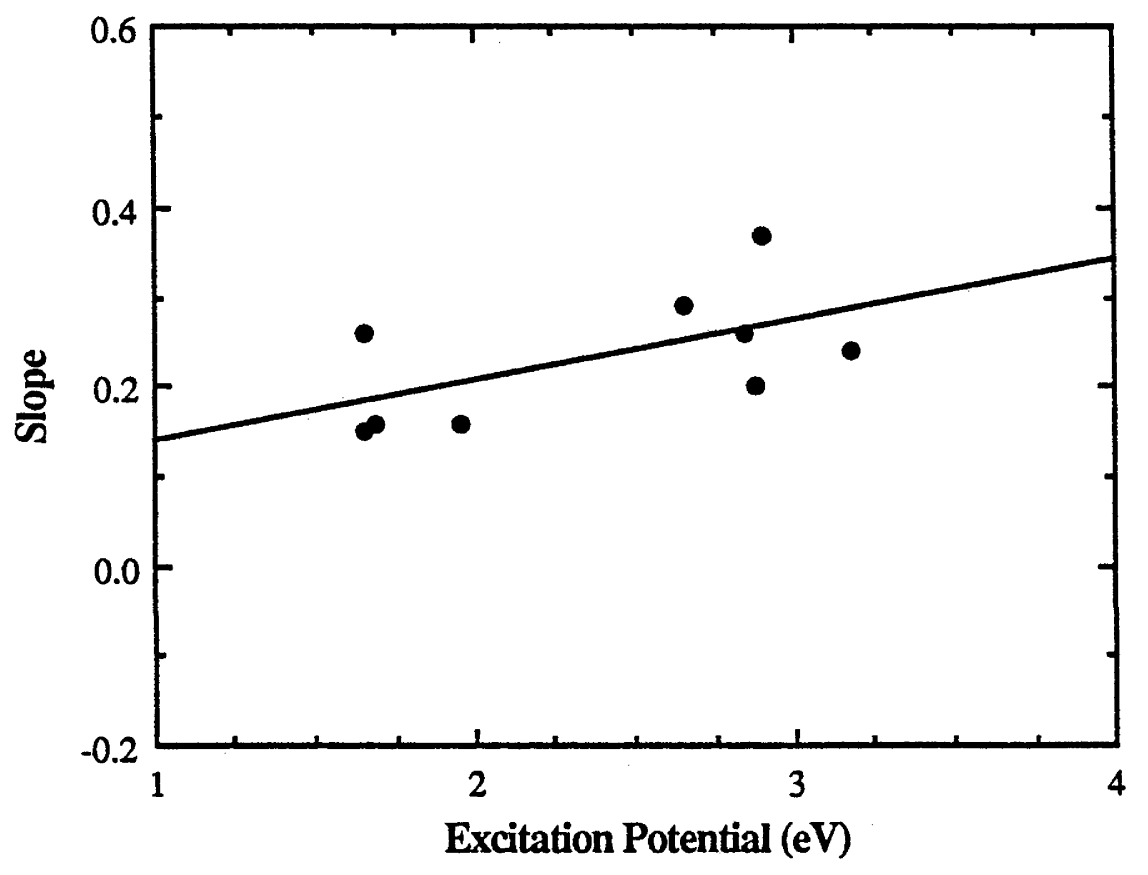


TABLE 14

Heavy Metal Abundances Relative to Galactic H-H Objects

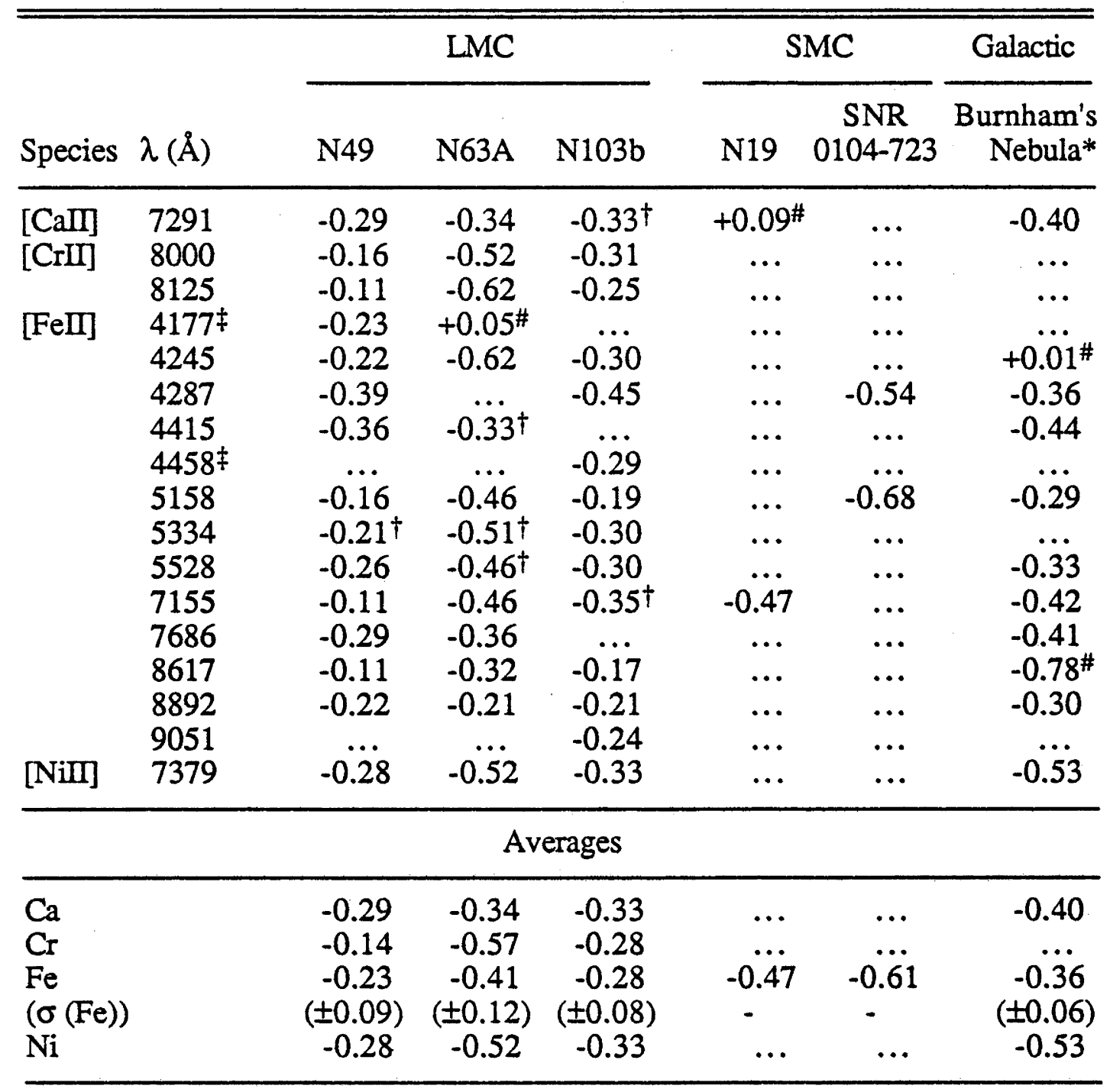

* Data derived from Solf, Böhm, and Raga 1988.

$\dagger$ Data derived from Danziger and Leibowitz 1985.

$\ddagger$ These wavelengths were only given half-weight due to poor statistics.

\# Not included in determining mean abundance. 
from Table 12 , is also very small at better than \pm 0.1 dex. This variation is smaller than that for our sample of supergiants from Paper I. In addition, the mean Fe abundance in the LMC, as derived from the SNRs, is the same as that derived from the supergiants (fortuitously considering the errors and the small samples involved). This gives us greater confidence that the values we are measuring in the SNRs are indeed the heavy metal abundances, thus lending added support to the conclusions of Brugel, Böhm and Mannery (1981) that grain depletion is unimportant. In fact this result is highly significant, since it allows us to connect the abundance scales derived from supergiants to those derived from H II regions, via the SNRs, for the first time.

The Fe abundance in the SMC is difficult to determine from SNRs due to the extreme weakness of the lines and the faintness of the objects available for study in that galaxy. As may be seen from Table 14, the two SNRs we observed in the SMC yielded a total of only three $\mathrm{Fe}$ lines with reasonable measures. The standard deviation from the mean SMC iron abundance quoted in Table 12, is therefore derived from the three measured lines rather than from the averages of the two objects (which would be meaningless). Considering the difficulties, the correspondence between the SMC iron abundances derived from the SNRs and the supergiants (from Paper I) is surprisingly good, and the small difference is well within experimental error.

There is some confusion in the literature over whether the line at $8000 \AA$ is due $[\mathrm{CrII}]$ or $[\mathrm{FeII}]$, with Solf, Böhm and Raga (1988) maintaining the latter identification. However, from the compilation of transition probabilities for [FeII] lines by Garstang (1962), we would expect [FeI] $\lambda 7999 \AA$ to be only one millionth as strong as [FeI] $\lambda 7637 \AA$, which is derived from the same upper level. Therefore we are confident that our identification of the line as [CrI] $\lambda 8000 \AA$ is accurate. The abundance of this element in the LMC is seen from Table 12 to be in good agreement with the results from the supergiants (see Paper I), thus further 
supporting our conclusion that we are measuring the heavy metal abundances in the Cloud ISM without serious systematic errors.

The $\mathrm{Ni}$ abundance in the LMC is also measured by us to be close to that derived from the supergiants (see Table 12), indeed the depletion with respect to the Sun is closer to that of $\mathrm{Fe}$ in the SNRs than in the supergiants, and therefore possibly more reliable. Our conclusion is based on a single line measured in all three of our objects. However, from Table 5 we see that this line is of reasonable strength (5-10\% of $I\left(\mathrm{H}_{\beta}\right)$ ), it is free of contamination (see Fig. $2 a$ ) in all but $\mathrm{N} 103 \mathrm{~b}$, and is therefore quite reliable. In the case of N103b the Ni line was only observed with FORS, and the lower resolution resulted in some blending with the strong line group near $7325 \AA$. The line was, however, easily deblended using the SPECTRE routines mentioned previously. Overall the error in the mean abundance is probably of the order $\pm 0.15-0.2$ dex, which is mainly due to the uncertainty in the Galactic standard.

Our conclusion of normal depletion of $\mathrm{Ni}$ with respect to Galactic $\mathrm{H}-\mathrm{H}$ objects is unrelated to the absolute $\mathrm{Ni}$ abundance. However our derived absolute abundances depend on the $\mathrm{H}-\mathrm{H}$ objects having close to solar $\mathrm{Ni}$ abundance. The work of Dennefeld (1986) (and others) indicates that this might not be true, and in fact $\mathrm{Ni}$ is overabundant relative to $\mathrm{Fe}$ in all the shocked objects studied in our own Galaxy as well as the LMC. The fact that this overabundance is so wide spread tends to militate against its reality, since it suggests that by chance, all the regions of the ISM studied through their emission due to shock excitation, have peculiar $\mathrm{Ni}$ abundances compared to the observed and well established "cosmic" abundances (we use "cosmic" and "solar" abundances interchangeably). This is clearly implausible, and the observation that the ratio of the line at $7379 \AA$ with any [Fem] line (see Dennefeld 1986) is relatively constant from object to object supports the conjecture that the line at $7379 \AA$ is due to $[\mathrm{FeII}]$ rather than [NiII], even though there is as yet no known candidate line. Alternative explanations, preferred by 
Dennefeld (1986), are that the population of $2 \mathrm{~F} \mathrm{Ni}^{+}$is selectively enhanced for some undetermined reason, or the collisional excitation coefficients are substantially in error. Both of these conjectures were tested by Henry and Fesen (1988) and found to be unlikely. They suggest that if [NiII] emission is produced in regions with densities of the order of $10^{5} \mathrm{~cm}^{-3}$ or more, the observations may be understandable, although the implied cooling times for some of the objects observed may be unrealistically short. We make no further comment on the issue as it is not our purpose here to investigate the problem, merely to warn that the basis for our assumption of solar abundances in $\mathrm{H}-\mathrm{H}$ objects is, in the case of $\mathrm{Ni}$, still questionable, as is the mere identification of the line at $7379 \AA$.

If we use the abundance analysis of Dennefeld (1986) simply as a check on our measurements for the the depletion of Fe and Ni in LMC supernova remnants relative to Galactic $\mathrm{H}-\mathrm{H}$ objects, we find that the systematic difference between our results and theirs, is less than 0.02 dex, with a random difference of less than \pm 0.1 dex. Taking into account the different samples of $\mathrm{H}-\mathrm{H}$ objects used in the two cases, this gives some justification for the methods we have used and errors we have quoted.

The Ca abundance of the LMC derived from our work is within the bounds of experimental error of the mean abundance derived from the supergiants. As in the case of $\mathrm{Ni}$, the depletion of $\mathrm{Ca}$ relative to solar abundance is more nearly the same as the Fe depletion in the sample of SNRs than in the supergiants, and is again the more plausible result. This result depends on just one line of [CaIf in each object, however, the line is strong $(15-30 \%$ of $I(\mathrm{H} \beta))$ and is uncontaminated by other lines (see Table 5 and Fig. 2a). As such, the quality of the derived abundance is quite good, although the error is around 0.2 dex due to uncertainties in the galactic standard. The result is significant since it means that dust grains must be destroyed in the shocked environment. Mere detection of any $\mathrm{Ca}$ lines rules out the degree of 
depletion of the element, through condensation on to dust grains, suggested by Spitzer and Jenkins (1975).

The measure of the line [CaII] $\lambda 7291 \AA$ in N19 is of poor quality due to the faintness of the object, the limited time we could afford to observe it, and the poor response of the IPCS in this part of the spectrum. The line was well observed in the FORS spectrum, however in this case it could not be resolved from the line group at $\lambda 7325 \AA$. The two spectra agree in the total emission from the lines at $\lambda 7325 \AA$ and $\lambda 7291 \AA$, but we would still need further evidence before we could have confidence in the derived $\mathrm{Ca}$ abundance quoted for N19 in Table 14.

In summary, we find that all the evidence points to the SNRs having heavy metal abundances, and in particular iron abundances, that are in very close agreement with those derived from the supergiants studied in Paper I. As a result, there is no need, at the present levels of accuracy, for a zero point shift to bring the scales of abundance from the two types of object into agreement with each other. This result is of great importance in future observations of galaxies for which both young supergiants and H II regions may be observable, but SNRs are not. Clearly, if we can be sure there is no zero point error, then the SNRs become redundant. If a zero point error had been found we would have been set the unenviable task of answering the question of why, and more importantly, should we expect the same error from galaxy to galaxy?

\section{OVERALL RESULTS}

In Figures $16 a$ and $16 b$ we have plotted the differences between the absolute abundance we derived from the SNRs and those we derived for the $\mathrm{H}$ II regions+supergiants (open circles) for the SMC and LMC respectively. The error bars, when present, represent the object-to-object scatter from the mean abundance 
Figure 16 A comparison between the abundances derived from SNRs and HIIregions $(\log [N(\mathrm{SNR}) / N(\mathrm{HII}$ region) $])$ for $(a)$ the SMC, and $(b)$ the LMC. The open circles represent the abundance differences between SNRs from this work and the HIII-regions from this work (or supergiants from Paper I for C and elements heavier than $\mathrm{Ar}$ ), plotted as a function of the atomic number $\mathrm{Z}$. For comparison, the filled circles represent the abundance differences of the HII-regions of Dufour (1984) compared to our own (log[N(Dufour)/N(this work)]). 

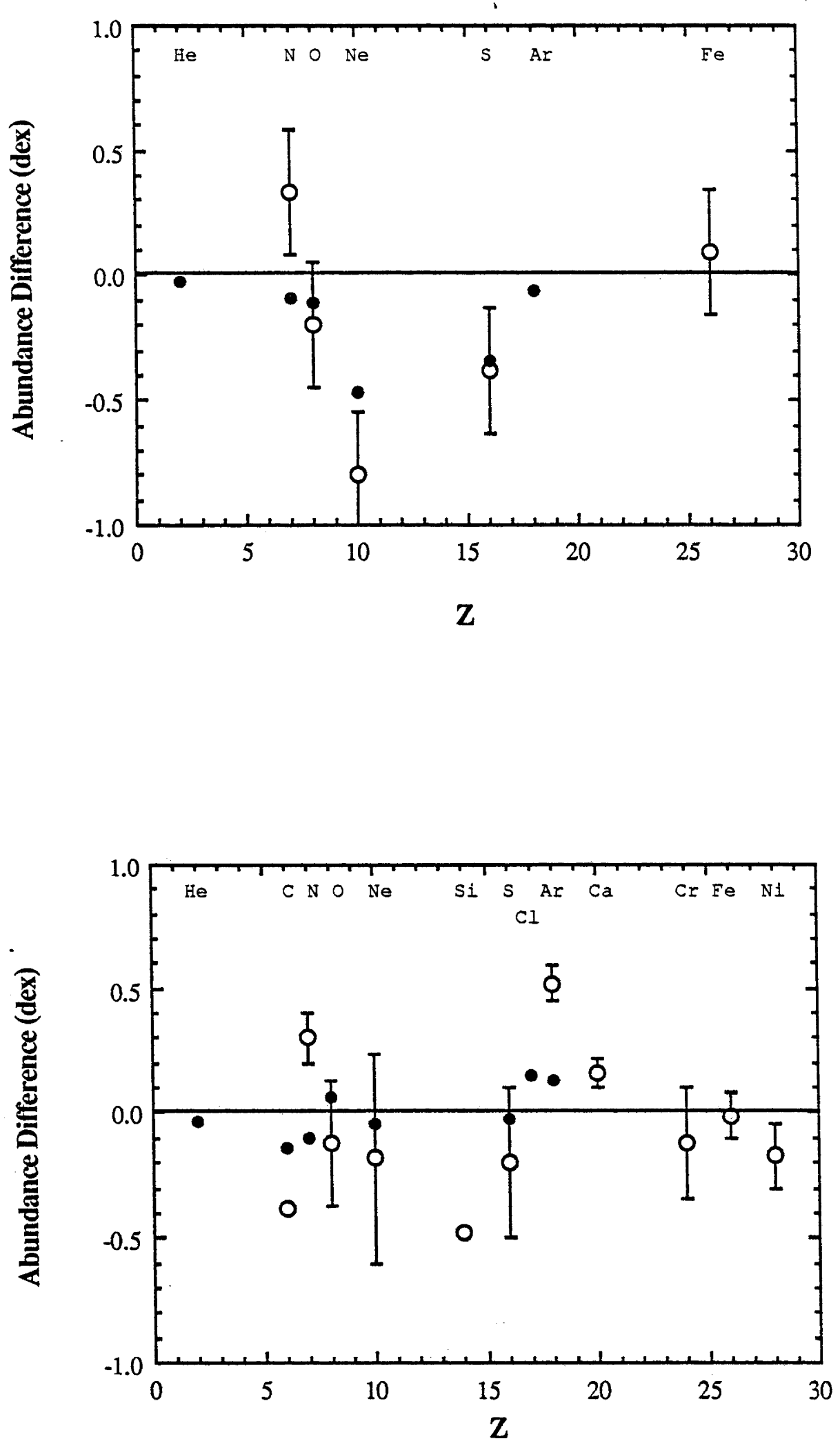
difference. Where less than three objects were used in determining the mean, the error bars were excluded. For comparison, the filled circles represent the mean differences between the $\mathrm{H}$ II region abundances derived from Dufour (1984) and those derived from $\mathrm{H}$ II regions in this work (and the supergiants in the case of carbon). Overall the agreement between the SNRs and the H II regions+supergiants is rather good. If we take our H II region+supergiant data as the standard, the main differences seem to be an over-estimate of the nitrogen abundances in SNRs from both Clouds, an overestimate of argon in the LMC, an under-estimate of neon in the SMC, and underestimates for carbon and silicon for one object in the LMC. The sulphur abundances seem reasonable when the results of Dufour (1984) are considered as well.

The derivation of the nitrogen abundances in the SNRs has already been discussed in some detail previously, and it became apparent that their reliability is somewhat uncertain. Until this problem is resolved in the future by more accurate models, we can not be certain that the apparent overabundance of N in the SNRs is real. Nevertheless, it may be remarked here that a similar effect was noticed by Dopita (1976) for the LMC, and by Dopita, Mathewson, and Ford (1977) for all the available data on $\mathrm{H}$ II regions, planetary nebulae, and SNRs at the time. The latter authors suggested that there may be an error in the $\mathrm{H} \mathrm{II}$ region abundances due to a systematic temperature difference between low and high excitation zones of the nebulae. If however, it turns out that there does exist a real abundance difference between SNRs and H II regions, we may have to consider the possibility that the shocked cloudlets have after-all been polluted by $\mathrm{CNO}$-processed material from the pre-supernova star. This may well be possible if the star was able to blow off a significant fraction of its atmosphere as a slow stellar wind. If all pre-supernova stars polluted in the same way, the correlations observed in Figures $13 a$ and $13 b$ would not be compromised. 
The disagreement in the neon abundances for the SMC apparent from Figure $16 a$, and the very large scatter observed in the SNR neon abundances for the LMC all point to the great difficulty in determining the abundances from just one or two [NeIII] lines. As mentioned previously, this line is particularly sensitive to the carbon and silicon abundances, since these elements compete as coolants with high excitation species like [NeII] and [OII] in the high temperature zone in which they are formed. Errors in the assumed carbon abundances in particular could result in as much as a 0.3 dex error in the derived Ne abundances. Further advances in our understanding of the $\mathrm{Ne}$ abundances will have to wait for observations of the prominent UV lines [NeIV] $\lambda \lambda 1601,2422,2425$ and [NeV] $\lambda 3426$.

The rather high value for the argon abundance derived from the SNRs in the LMC is somewhat more enigmatic. In most objects, both H II region and SNR, the abundances are determined from two quite well observed lines. Indeed, for one H II region the abundance was determined from two lines of [ArIV] as well as the lines of [ArII], and the agreement was good. So it seems unlikely that simple observational errors can account for the observed abundance difference. Looking further afield, we note that Binette et al. (1982) obtained a similar (0.4dex) argon overabundance for the Cygnus Loop compared to Galactic $\mathrm{H} I I$ regions. It is therefore likely that the apparent argon overabundance in SNRs compared with H II regions is a general phenomenon. It is, however, possible that the error is due to inaccuracies in the modeling of the SNRs since for these objects most of the argon is either neutral or singly ionized, while for photoionized nebulae, most of the argon is doubly ionized. The only conceivable test for this at present is to make observations of the prominent $\mathrm{Arm}$ line at $6.985 \mu \mathrm{m}$. Again, until such evidence is acquired, our conclusions regarding the anomalous abundance of this element in SNRs would be no more than guess work.

Finally, we consider the apparent underabundances in carbon and silicon, derived from the UV spectrum of N49, compared with the results from the 
supergiants. Silicon was rather poorly measured in only two supergiants, so that value is still, at present, highly questionable. The preliminary analysis of a single near-mainsequence B-star in the LMC by Reitermann et al. (1988), suggests a $\mathrm{Si}$ abundance of $-0.2 \pm 0.2$ dex relative to a Galactic standard ( 1 Her), which is in much better agreement with our SNR result. We would therefore place more reliance on the SNR value, although the evidence is still rather tenuous.

In Figures $17 a$ and $17 b$ we present the abundance differences between objects in the SMC and LMC respectively, and the Sun. In these diagrams, the filled circles represent the abundance differences for both the H II regions from this work, and the supergiants from Paper I. There is no ambiguity in this presentation since the elements obtained from the two types of object do not overlap. The filled triangles represent the abundance differences for the H II regions of Dufour (1984), while the open circles represent our results for the SNRs.

These may be compared with Figures $18 a$ and $18 b$, which are the same as for Figure 17, except the objects are differenced with the "local" ISM, rather than the Sun, and additional results have been included (open squares) which represent the SNRs differenced with the Cygnus Loop. For the rest, the $\mathrm{H}$ II regions (filled circles) and the light elements (He to Ar) in the SNRs (open circles) are differenced with the abundances derived by Shaver et al. (1983) for the "local" ISM from H II regions, and with the carbon abundances derived from the UV observations of Orion reported by Dufour, Shields, and Talbot (1982) (see Table 12). The supergiants (also filled circles) are differenced with Canopus, the Galactic supergiant standard used in Paper I, while the heavier elements from the SNRs are again differenced with the Sun.

The carbon depletion from solar abundance in the LMC, as seen in Figure $17 b$, is very strong indeed compared to that of iron (the dashed line). However from Figure $18 b$ it seems that the depletion of carbon in the supergiants, relative to the "local" ISM, is no more than that of iron, a result that is supported by the work 
Figure 17 Depletion in elemental abundances for $(a)$ the SMC, and (b) the LMC, relative to the Sun. The filled circles represent the combined data from the HII-regions of this work, and the supergiants of Paper I, relative to the Sun. The open circles represent our results for the SNRs, while the open triangles represent the data of Dufour (1984). 

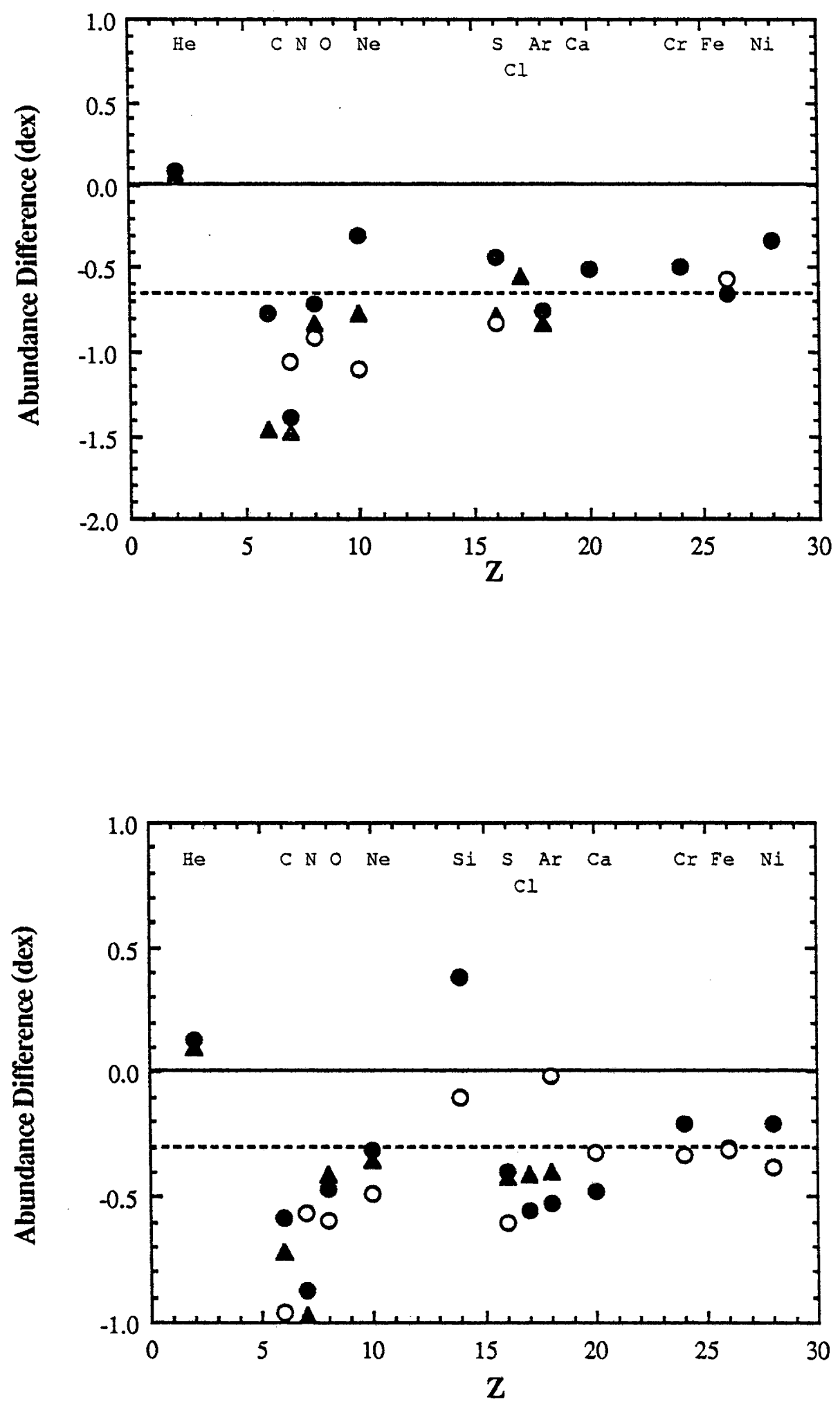
Figure 18 Depletion in elemental abundances for (a) the SMC, and (b) the LMC, relative to the local ISM. The Filled circles represent our data for HII-regions relative to that of Shaver et al. 1983 for HII-regions in the local solar region, combined with data from the supergiants of Paper I relative to Canopus. The filled triangles are from Dufour (1984) relative to Shaver et al. (1983) or Dufour (1982) for carbon. The open circles represent the SNRs from this work compared with either Shaver et al. (1983), the carbon abundance for Orion from Dufour (1982), or the Sun, depending on the element. Finally, the open squares represent the SNRs from this work relative to the Cygnus Loop for those elements they have in common. 

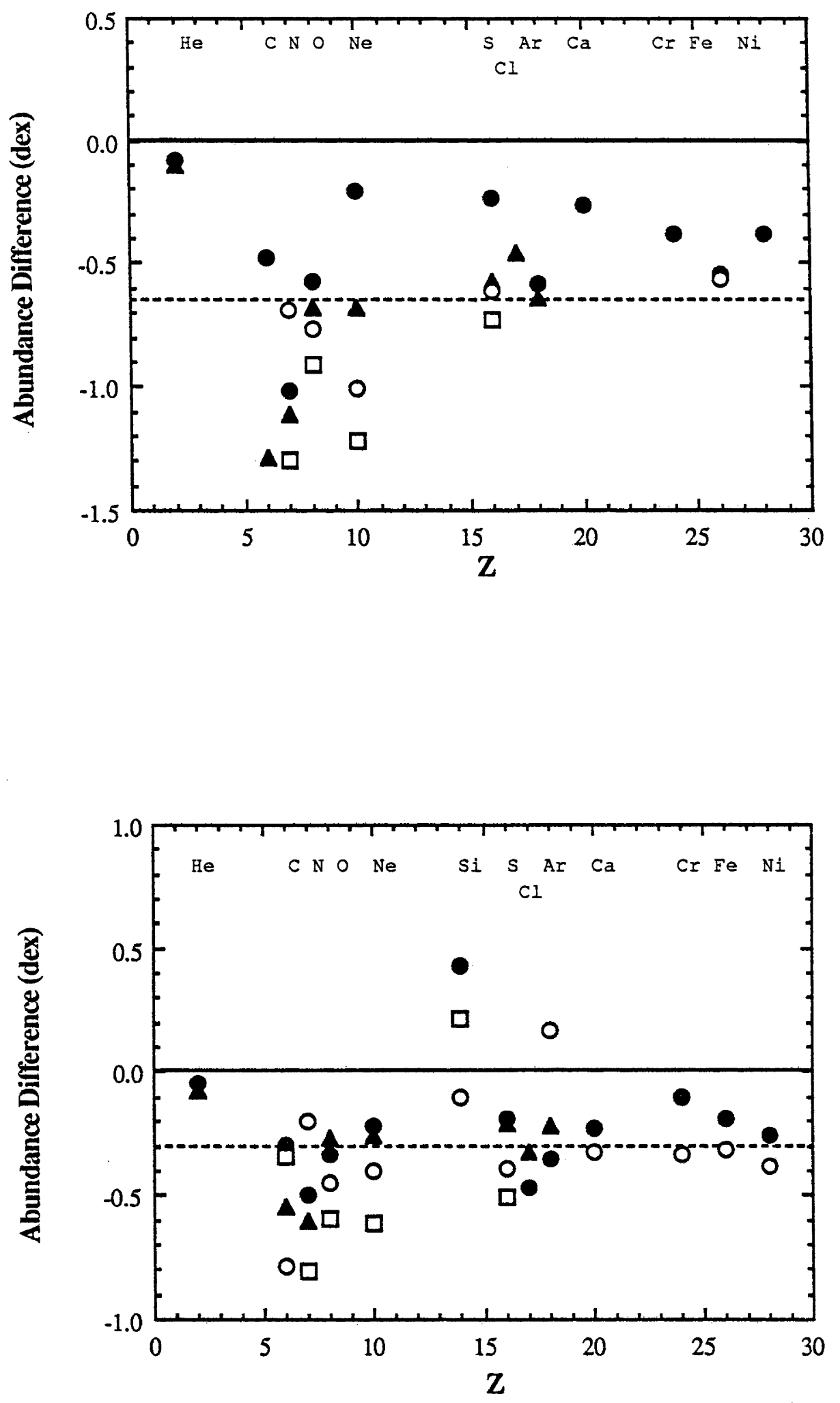


\section{SUMMARY}

In this paper we publish some of the most detailed spectra for $\mathrm{H} I I$ regions and supernova remnants in the Magellanic Clouds, to appear in print to date, plus detailed spectra of four Herbig-Haro Objects from our own Galaxy. We have made observations beyond the optical region of the spectrum, to a limit of $\sim 1.1 \mu \mathrm{m}$, thus allowing measurements of many more useful lines, in particular [SII], the most abundant ionization species of $\mathrm{S}$ in $\mathrm{H}$ II regions, and the heavy metals $\mathrm{Ca}, \mathrm{Cr}, \mathrm{Fe}$, and $\mathrm{Ni}$ in the SNRs. We have detected both resonance and forbidden lines of $\mathrm{Ca}$ in one $\mathrm{H} \mathrm{II}$ region, and in another we observe an intercombination line of $\mathrm{MgI}]$ at $4571 \AA$. We also report the first spectrum taken of a SNR in the SMC (SNR0104723) other than the better known N19. The spectrum for this object is in fact quite similar to that of $\mathrm{N} 19$, and includes a detection of the $\mathrm{Na} \mathrm{D}$ doublet. We note that the LMC nebulosity N179A is of very low excitation and suggests the possible presence of shock phenomena. We suggest that it may be that the gas is being shock excited by the wind from an embedded Wolf-Rayet star.

We performed the first consistent detailed modeling of both SNRs and H II regions in the Magellanic Clouds. This revealed that the elemental abundances in the $\mathrm{H}$ II regions are in substantial agreement with the literature, though with a slightly high value determined for $\mathrm{Ne}$ in the SMC. A high $\mathrm{Ne} / \mathrm{O}$ ratio may indicate that oxygen, together with the refractory elements, is depleted through dust formation. There is a suggestion of an enhanced spread in oxygen abundances in the LMC which may be indicating a degree of incomplete mixing in the ISM.

Detailed modeling of the SNRs resulted in abundance measures in good agreement with those from $\mathrm{H}$ II regions, from this work and the literature, thus confirming the overall accuracy of the generalized modeling code MAPPINGS for both photoionized and shock excited nebulae. For the elements heavier than Ar, a differential analysis was performed between the SNRs and the Galactic $\mathrm{H}-\mathrm{H}$ 
objects. This analysis revealed that there is no evidence for grain depletion in either Cloud SNRs or Galactic H-H objects. More importantly, it showed that there is no measurable zero-point error between the $\mathrm{Fe}$ abundances determined from supergiants and those determined from SNRs. This was supported by the results from the additional heavy metals $\mathrm{Ca}, \mathrm{Cr}$, and $\mathrm{Ni}$, and allows us for the first time to bring together the two traditionally separated abundance scales of the $\mathrm{H}$ II regions and the supergiants, into one comprehensive system.

The overall abundance pattern for the light elements is of great importance in the determination of the star formation history in the Clouds. When compared with the Sun, the Clouds show a systematic decrease in elemental abundances for decreasing atomic number. When, however, they are compared with the present local ISM, no such decrease in abundance is observed. The implication is that the star formation history of the Clouds has been little different from that of the present ISM, but significantly different from the medium out of which the Sun was formed.

Thanks to all those who have helped in making the observations, and to the Directors and staff of both Mount Stromlo and Siding Spring Observatories and the Anglo Australian Observatory, for the use of their facilities. The Australian Time Allocation Committee has been generous in their support for this project, and are gratefully acknowledged. Thanks also to Peter Wood and Bernard Pagel for their helpful comments and suggestions. Finally, one of us (S.C.R.) would like to acknowledge the financial support received from a Commonwealth Postgraduate Scholarship during the course of this work. 


\section{REFERENCES}

Allen, D. A. 1979, M.N.R.A.S., 186, 1P.

Allan, R. J., Clegg, R. E. S., Dickinson, A. S., and Flower, D. R. 1988, Ap. J., 235, 1245 .

Aller, L. H., Czyzak, S. J., Keyes, C. D., and Boeshaar, G. 1974, Proc. Nat. Acad. Sci. USA, 71, 4496.

Aller, L. H., Keyes, C. D., and Czyzak, S. J. 1979, Proc. Nat. Acad. Sci. USA, 76,1525 .

Auer, L. H., and Mihalas, D. M. 1972, Ap. J. Suppl., 24, 193.

Azzopardi, M., and Breysacher, J. 1980, Astr. Ap. Suppl., 39, 19.

Baker , J. G., and Menzel, D. H. 1938, Ap. J., 88, 52.

Benvenuti, P., Dopita, M. A., and D'Odorico, S. 1980, Ap. J., 238, 601.

Binette, L., Dopita, M. A., D'Odorico, S., and Benvenuti, P. 1982, Astr. Ap., $115,315$.

Binette, L., Dopita, M. A., and Tuohy, I. R. 1985, Ap. J., 297, 476.

Blair, W. P., Kirshner, P., and Chevalier, R. A. 1981, Ap.J., 247, 879. 1982, Ap. J., 254, 50.

Böhm, K. -H., Perry, J. F. and Schwartz, R. 1973, Ap. J., 179, 149.

Böhm, K. -H., Schwartz, R. D., and Siegmund, W. A. 1974, Ap. J., 193, 353.

Böhm, K. -H., Siegmund, W. A., and Schwartz, R. D. 1976, Ap. J., 203, 399.

Boksenberg, A. 1972, in Proceedings ESO/CERN Conference on Auxiliary Instruments or Large Optical Telescopes, ed. S. Laustsen and A. Reiz (Geneva: European Southern Observatory), p. 295.

Boksenberg, A., and Burgess, D. E. 1973, in Proceedings Symposium on Astronomical Observation with Television-Type Sensors, ed. J. W. Glaspey and G. A. H. Walker (Vancouver: University of British Columbia), p. 21.

Brocklehurst, M. 1971, M.N.R.A.S., 153, 471. 
Brugel, E. W., Böhm, K. H. and Mannery, E. 1981, Ap. J. Suppl., 47, 117.

Butler, S. E., and Raymond, J. C. 1980, Ap. J., 240, 680.

Cameron, A. G. W. 1982, in Essays in Nuclear Astrophysics, ed. C. A. Barnes, D. D. Clayton, and D. N. Schraam (Cambridge University Press), p. 23.

Choe, S.-U., Böhm, K. H., and Solf, J. 1985, Ap. J., 288, 338.

Chu. Y.-H., and Kennicutt, R. C. Jr. 1988, Astr. J., 95, 1111.

Clegg, R. E. S. 1987, M.N.R.A.S., 229, 31P.

Contini, M. 1987, Astr. Ap., 174, 5.

Cowie, L. L. 1978, Ap. J., 225, 887.

Cox, D. P., 1972, Ap. J., 178, 143.

Cox, D. P., and Raymond, J. C. 1985, Ap. J., 298, 651.

Danziger, I. J., and Leibowitz, E. M. 1985, M.N.R.A.S., 216, 365.

Davies, R. D., Elliott, K. H., and Meaburn, J. 1976, Mem. R. Astr. Soc., 81,89.

Dennefeld, M. 1986, Astr. Ap., 157, 267.

Dennefeld, M., and Kunth, D. 1981, Astr. J., 86, 989.

Dennefeld, M., and Stasinska, G. 1983, Astr. Ap., 118, 234.

Dickel, H., Aller, L. H. and Faulkner, D. J., 1964, IAU Symposium 120, The Galaxy and the Magellanic Clouds, ed. F. J. Kerr and A. W. Rodgers, (Australian Academy of Sciences), p. 294.

Dopita, M. A. 1976, Ap. J., 209, 395. . 1977, Ap. J. Suppl., 33, 437. . 1978, Ap. J. Suppl., 37, 117. . 1979, Ap. J. Suppl., 40, 455. . 1982, in Supernovae: A Survey of Current Research, ed. M. J. Rees and R. J. Stoneham (Dordrecht: Reidel), p. 483.

Dopita, M. A., Binette, L., D'Odorico, S, and Benvenuti, P. 1984, Ap. J., 276, 653.

Dopita, M. A., Binette, L., and Schwartz, R. D. 1982, Ap. J., 261, 183. 
Dopita, M. A., D'Odorico, S., and Benvenuti, P. 1980, Ap. J., 236, 628.

Dopita, M. A., and Evans, I. N. 1986, Ap. J., 307, 431.

Dopita, M. A., Mathewson, D. S. and Ford, V. L. 1977, Ap. J., 214, 179.

Dopita, M. A., and Tuohy, I. R. 1984, Ap. J., 282, 135.

Dopita, M. A., Tuohy, I. R., and Mathewson, D. S. 1981, Ap. J. (Letters), 248, L105.

Draine, B. T., and Lee, H. M. 1984, Ap. J., 285, 89.

Draine, B. T., and Salpeter, E. E. 1979, Ap. J., 231, 438.

Dufour, R. J. 1975, Ap.J., 195, 315. . 1984, in IAU Symposium 108, Structure and Evolution of the

Magellanic Clouds, ed. S. van den Bergh and K. S. de Boer (Dordrecht: Reidel), p. 353.

Dufour, R. J., and Harlow, W. V., 1977, Ap. J., 216, 706.

Dufour, R. J., and Killen, R. M. 1977, Ap. J., 211, 68.

Dufour, R. J., Schiffer, F. H., and Shields, G. A., 1984, in The Future of Ultraviolet Astronomy Based on Six Years of I.U.E. Research, N.A.S.A. Conf. Publ., p. 111.

Dufour, R. J., Shields, G. A., and Talbot, R. J. 1982, Ap. J., 252, 461.

Evans, I. N. 1986, Ap. J., 309, 544.

Evans, I. N., and Dopita, M. A. 1985, Ap. J. Suppl., 58, 125.

Garstang, R. H. 1962, M.N.R.A.S., 124, 322.

Hartigan, P. 1987, Ph.D. (Australian National University).

Hartigan, P., Raymond, J., and Hartmann, L. 1987, Ap. J., 316, 323.

Hartmann, L., and Raymond, J. C. 1984, Ap. J., 276, 560.

Henize, K. G. 1956, Ap. J. Suppl., $2,315$.

Henry, R. B. C., and Fesen, R. A. 1988, Ap. J., 329, 693.

Herbig, G. H. 1951, A.J., 113, 697.

Hummer, D. G., and Mihalas, D. M. 1970, M.N.R.A.S., 147, 339. 
Kaler, J. B. 1976, Ap. J. Suppl., 31, 517.

Lasker, B. M. 1981, Pub. A.S.P., 93, 422.

Leibowitz, E. M., and Danziger, I. J. 1983, M.N.R.A.S., 204, 273.

Long, K. S., Helfand, D. J., and Grabelsky, D. A. 1981, Ap. J., 248, 925.

Mathewson, D. S., Ford, V. L., Dopita, M. A., Tuohy, I. R., Mills, B. Y. and Turtle, I. R. 1984, Ap. J. Suppl., 55, 189.

Matteucci, F. 1986, Pub. A.S.P., 98, 173.

Mendoza, C. 1983, IAU Symposium 103: Planetary Nebulae, ed. D. R. Flower (Dordrecht: Reidel), p. 143.

Mendoza, C., and Zeippen, C. J. 1982, M.N.R.A.S., 198, 127.

Miller, J. S., 1968, Ap. J. (Letters), 154, L57.

Mohler, O. C., Pierce, A. K., McMath, R. R., and Goldberg, L. 1950, Photometric Atlas of the Near Infra-red Solar Spectrum 18465 to 125,242 , (Pontiac: Ann Arbor University of Michigan Press).

Moore, C. E. 1945, A Multiplet Table of Astrophysical Interest, Contr. Princeton Univ. Obs., No. 20 (RMT).

Oke, J. B., 1974, Ap. J. Suppl., 27, 21.

Oke, J. B., and Gunn, J. E. 1983, Ap. J., 266, 713.

Osterbrock, D. E. and Dufour, R. J. 1973, Ap. J., 185, 441.

Pagel, B. E., Edmunds, M. G., Fosbury, R. A. E., and Webster, B. L. 1978, M.N.R.A.S., 184, 569.

Peimbert, M. and Torres-Peimbert, S., 1974, Ap. J., 193, 327. 1976, Ap. J., 203, 581.

Pelat, D., Alloin, D., and Fosbury, R. A. E., 1981, M.N.R.A.S., $195,787$.

Raymond, J. C. 1979, Ap. J. Suppl., 39, 1.

Raymond, J. C., Hartigan, P., and Hartmann, L. 1988, Ap. J., 326, 323.

Reitermann, A., Stahl, O., Wolf, B., Baschek, B., and Scholz, M. 1988, talk given at the IAU General Assembly, Baltimore. 
Rosado, M. 1986, Astr. Ap., 160, 211.

Russell, S. C., and Bessell, M. S., 1989, Ap. J. Suppl. , (in press, Paper I).

Schwartz, R. D., 1975, Ap. J., 195, 631. . 1976, Pub. A.S.P, 88, 159.

Schwartz, R. D., and Dopita, M. A. 1980, Ap. J., 236, 543.

Seab , C. G., and Shull, J. M. 1983, Ap. J., 275, 652.

Searle, L. 1971, Ap. J., 168, 327.

Shaver, P. A., McGee, R. X., Newton, L. M., Danks, A. C., and Pottasch, S. R. 1983, M.N.R.A.S., 204, 53.

Shields, G. A., and Searle, L. 1978, Ap. J., 222, 821.

Shull, J. M. 1979, Ap. J., 231, 534.

Shull, P. Jr. 1983a, Ap. J., 275, 592. . 1983b, Ap. J., 275, 611.

Shull, J. M., and Draine, B. T. 1987, in Tetons Conference on Physical Processes in the Interstellar Medium, ed D. J. Hollenbach, H. A. Jr. Thronsen (Dordrecht: Reidel), p. 283.

Shull, J. M., and McKee, C. F. 1979, Ap. J., 227, 131.

Solf, J., Böhm, K. H., and Raga, A. 1986, Ap. J., 305, 795.

—. 1988, Ap. J., 334, 229.

Spite, M., Barbuy, B., and Spite, F. 1989, (preprint).

Spitzer, L., Jr., and Jenkins, E. B. 1975, Ann. Rev. Astr. Ap., 13, 133.

Stasinska, G. 1980, Astr. Ap., 84, 320. 1982, Astr. Ap. Suppl., 48, 299.

Strom, S. E., Grasdalen, G. L., and Strom, K. M. 1974, Ap. J., 191, 111.

Taylor, B. J. 1984, Ap. J. Suppl., 54, 259.

Warren, W. H. Jr., and Hesser, J. E. 1978, Ap. J. Suppl., 36, 497.

Webster, B. L., 1976, M.N.R.A.S., 174, 513.

Whitford, A. E. 1958, A.J., 63, 201. 


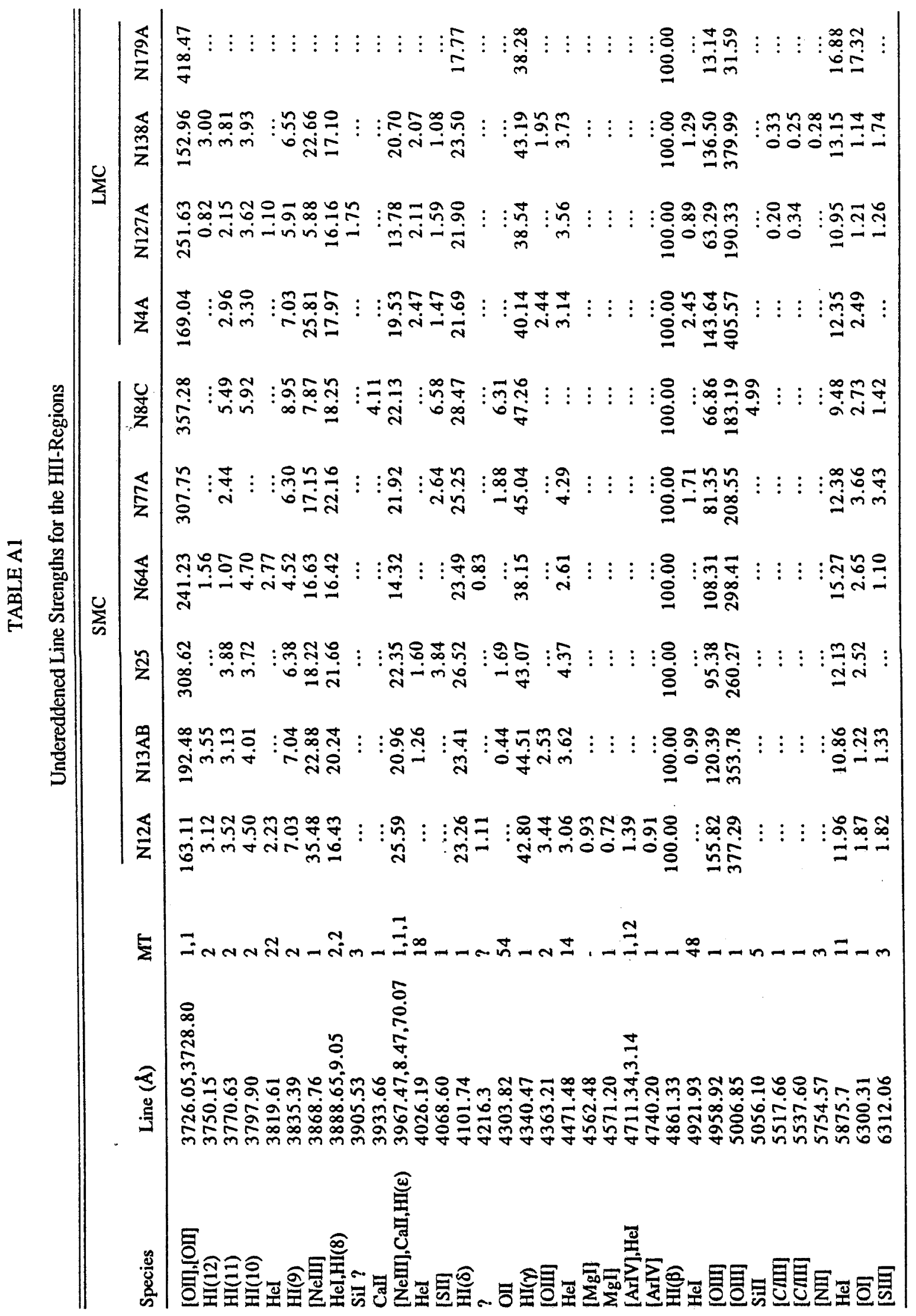




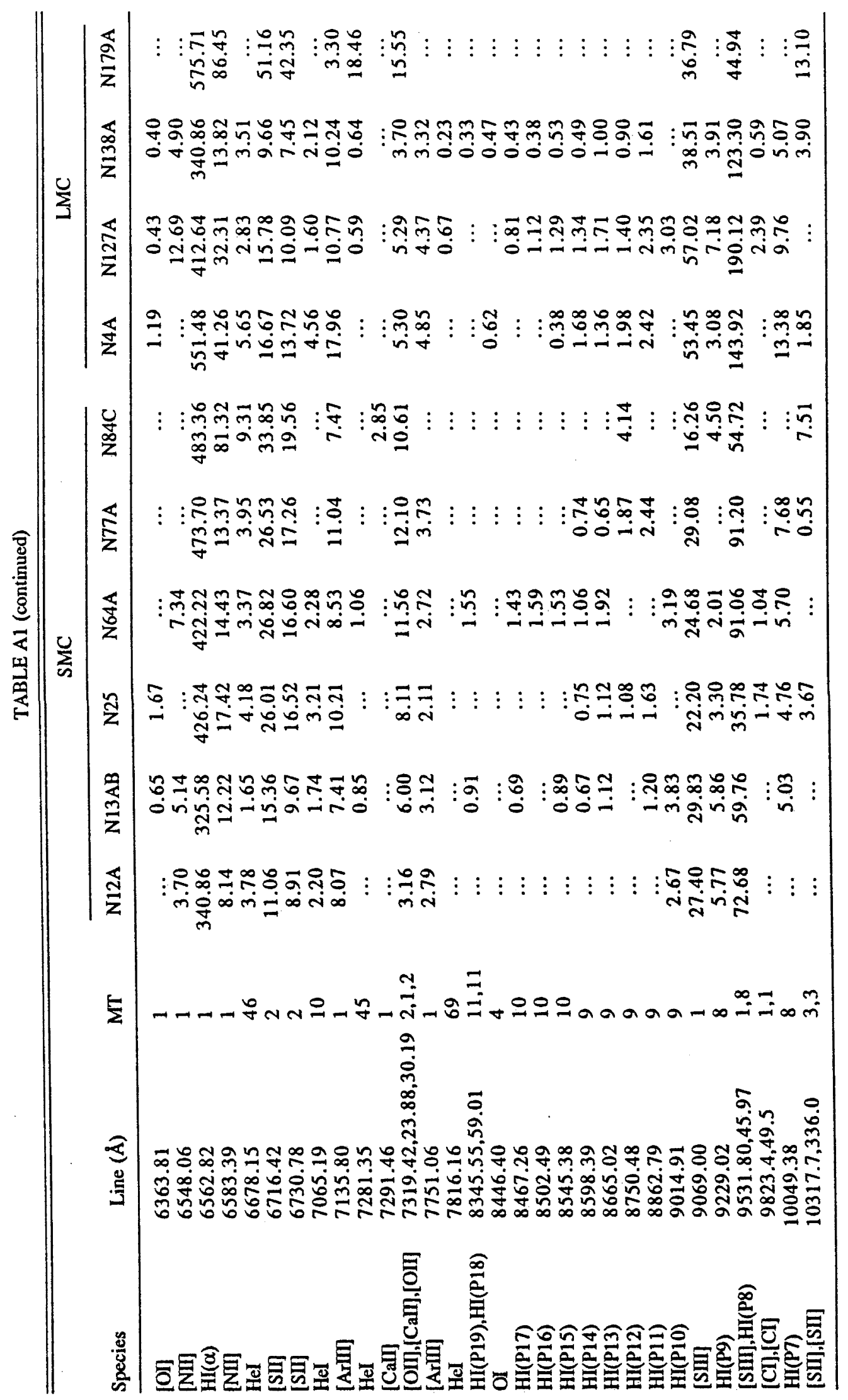




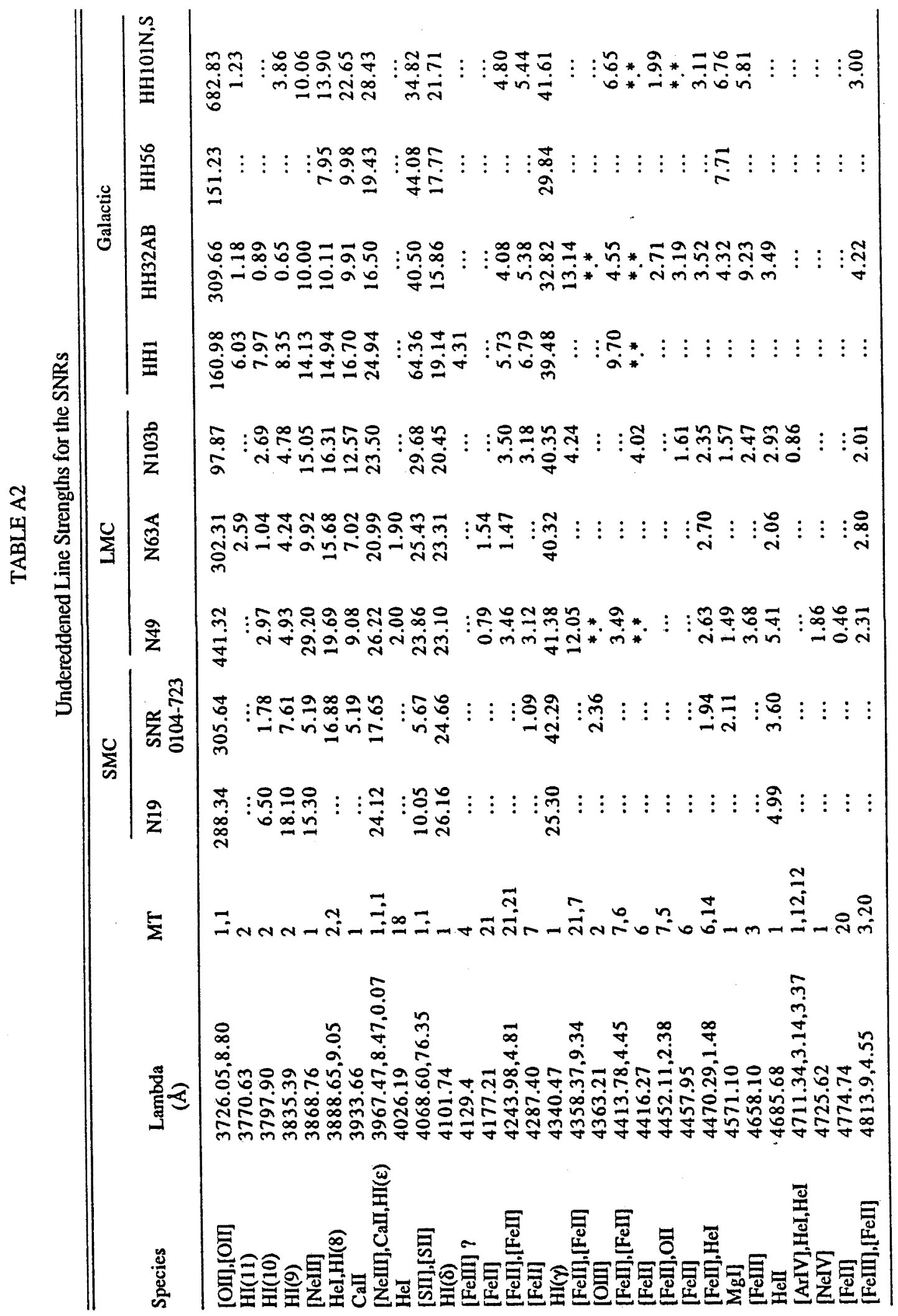




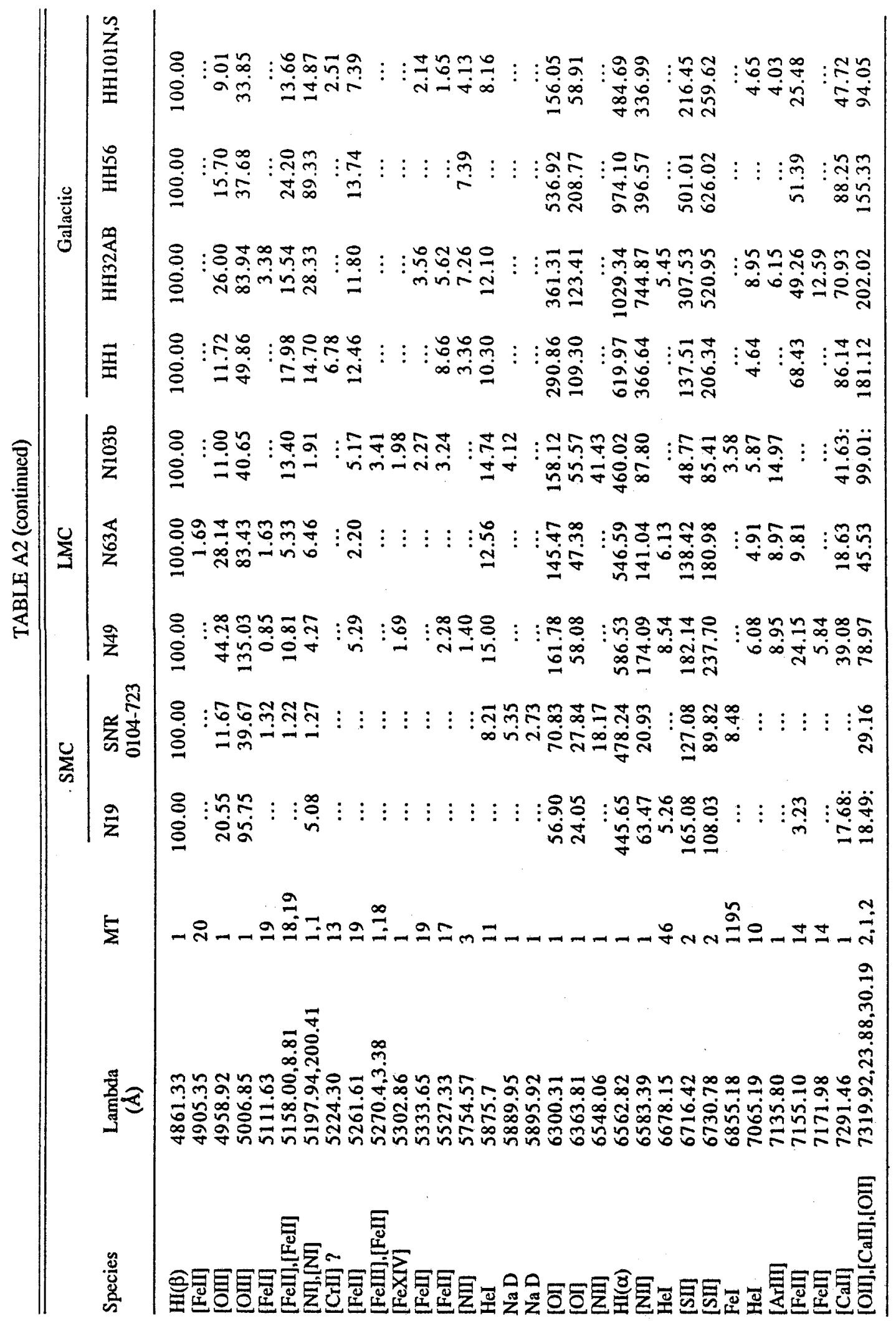




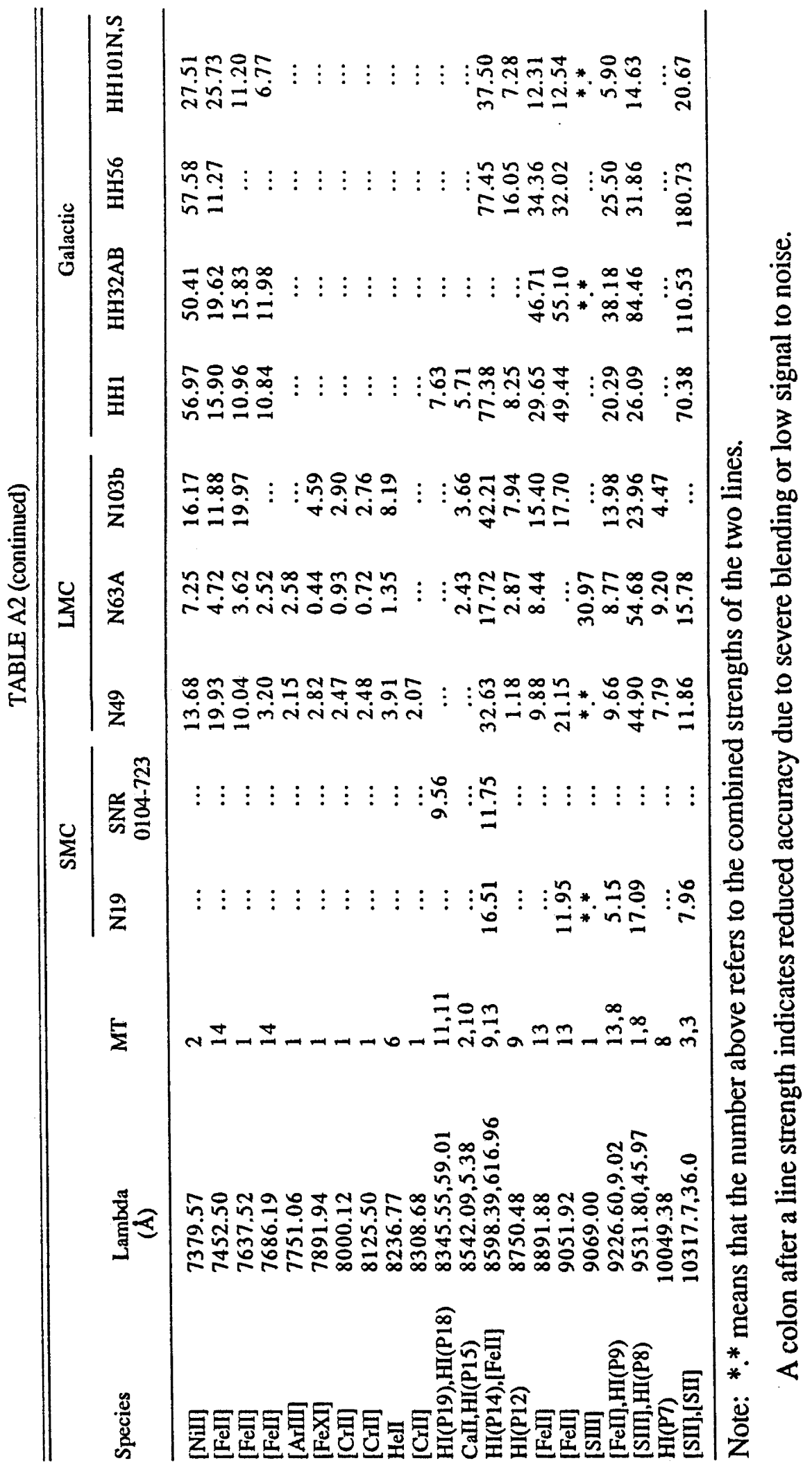




\title{
Abundances of the Heavy Elements in the Magellanic Clouds III. Interpretation of Results
}

\author{
Stephen C. Russell, and Michael A. Dopita
}

Mount Stromlo and Siding Spring Observatories, Institute of Advanced Studies, The Australian National University. 


\begin{abstract}
This paper presents chemical and structural evolutionary models for the Magellanic Clouds assuming bimodal star formation and gas infall. The models are discussed in relation to the observed chemical abundances of the Clouds and are compared with our own Galaxy. The detailed abundances derived from previous work are investigated for any obvious trends with metallicity or differences compared with the Galaxy. Considering all the data, conclusions are drawn on the possible star formation histories of the Magellanic Clouds and the implications for our own Galaxy.
\end{abstract}

The following conclusions were reached in this work. The interstellar medium (ISM) of the LMC has a mean metallicity 0.2 dex lower than the local Galactic ISM, and the metallicity of the SMC is 0.6 dex lower. However, the interstellar media of both the Magellanic Clouds and the Galaxy have significantly non-solar elemental ratios. This is most evident when considering the lightest and the heaviest of the elements. The $s$-process appears to have been as effective in the Magellanic Clouds as in the local ISM for elements as massive as $\mathrm{Zr}(\mathrm{Z}=40)$ for the SMC, and as massive as $\mathrm{Ba}(\mathrm{Z}=56)$ for the $\mathrm{LMC}$, but to have much reduced effectiveness above these atomic numbers. The r-process elements appear to be overabundant in the Clouds, relative to the Sun, with a distribution that indicates that the elements heavier than $\mathrm{Ba}$ in the $\mathrm{LMC}$, and heavier than $\mathrm{Zr}$ in the SMC, are produced only by the $r$-process.

Of the light elements, carbon appears to be of normal abundance in the Cloud stars relative to the iron abundance, which is in contradistinction to the carbon abundances in the H II regions which appear to be anomalously low. The overall distribution of the lightest elements in the Clouds shows a gradual falling off in the abundances, relative to the Sun, as the atomic number decreases. This is seen not to 
be the case when the abundances are compared with the local interstellar medium, and suggests that the present interstellar medium in the vicinity of the Sun is material that has fallen in from further out. 


\section{INTRODUCTION}

The Magellanic Clouds are in many ways easier systems to interpret than our own Galaxy. Their key advantages are that they may be viewed in their entirety with very little dust obscuration, either from the Galaxy or internally, and their distances are well established. They are also close enough for their population of gaseous emission objects (H II regions, supernova-remnants (SNRs), and planetary nebulae) to be studied in great detail, and in addition, with the present generation of sensitive detectors, the complete populations of supergiants in each Cloud are accessible for detailed analysis. Finally, they appear to be somewhat simpler systems, in that they have no well defined halo or disc, and they are relatively well mixed by their central bars (see Pagel et al. 1978) so that an abundance analysis at any one point should reflect the global abundances of the whole Cloud. Dynamically, however, it must be remembered, the Clouds are quite complex, since we observe them at a time when the SMC is being severely disrupted by the gravitational interaction with the Galaxy (see Mathewson and Ford 1984), and a "bridge" of $\mathrm{HI}$ gas is observed to connect the two Clouds.

In our two previous papers in this series, Russell and Bessell (1989 Paper I), and Russell and Dopita (1989 Paper II), we reported the results of a program of observations intended to establish the first consistent set of global abundances for both Clouds, covering the range of elements from the very lightest $(\mathrm{He}, \mathrm{C}, \mathrm{N}, \mathrm{O})$, to some of the heaviest (Nd, Sm) observable. Paper I dealt with the detailed abundance analyses of high-dispersion spectra of samples of medium to low luminosity F-supergiants in both Magellanic Clouds. Paper II described our analysis of the emission spectra of samples of bright H II regions and SNRs in both Clouds, using the general-purpose modeling code MAPPINGS (see Binette, Dopita and Tuohy 1985, and Evans and Dopita 1985). The SNRs were used to provide an overlap between the otherwise disparate sets of abundances derived from 
supergiants and $\mathrm{H} \mathrm{II}$ regions. In this way we were able to ensure that the sets of abundances we derived from all sources, for each Cloud, were consistent over the whole range of atomic mass.

In this paper we attempt to interpret the results of the previous two papers in terms of the star formation histories of the Magellanic Clouds, and their relations with each other and with the Galaxy. In § II we describe the models we use to explore the star formation histories and associated elemental enrichment in each of the Clouds. In § III we discuss the elemental abundance patterns we have found in the Clouds and compare them with the models described in $\S \mathrm{II}$, and with results from the Galaxy. All the results are brought together in §IV in an attempt to understand the star formation histories of the Clouds, and the possible implications our results might have for the history of the Galaxy. Finally, in $\S \mathrm{V}$ we summarize our findings and make suggestions for some of the work that needs to be done in this relatively new field of research.

\section{STAR FORMATION MODELS}

\section{a. The Structural Evolution}

We present here evolutionary models for the SMC and the LMC and compare the results with the model for the Galaxy derived by Dopita (1989) using the same code. Since there is no reason to suppose that the solar neighborhood is in any way peculiar in its properties, there is a reasonable hope that an evolutionary model that can account for the properties of the local environment, would be capable of accounting for the properties of external galaxies as well.

To this end, a modeling code was developed along the lines described by Matteucci and Tornambé (1985) and Matteucci and Greggio (1986). The code calculates at each time step, the total amount of infallen gas; the number of stars that 
are formed within each $0.03 M_{\odot}$ log mass bin, over a specified range of masses (the models being insensitive to the exact mass limits); the number of stars in each mass bin that die and the gas they return to the ISM, and the remnants they leave behind in the form of white dwarfs or neutron stars; and the enrichment or dilution of the ISM that occurs through the ejection of both processed and unprocessed gas from each stellar mass. Any ejected matter is assumed to be made available for further star formation during the following time step. Variation of the yield with metallicity for the massive stars is taken into account, but radial flows are not. The end points for the models are constrained principally by the ratio of the gas mass to the total mass, the age of the system, the peak in the star formation rate, and the oxygen to iron ratio.

We assume that star formation takes place in two quite separate environments. The consequence of this is a bimodal form to the initial mass function (IMF) $\psi(\mathrm{M})$ (the number of stars born per $\mathrm{kpc}^{2}$ per year per [log $\left.M / M_{\odot}\right]$ ), similar to those proposed by Güsten and Mezger (1983), Larson (1986), and Wyse and Silk (1987). The high mass star-formation applies above $\sim 1 M_{\odot}$ and determines the chemical enrichment of the ISM, while the low mass star-formation applies below $1 M_{\odot}$ and serves only to lock up gas in long lived stars. The ratio of the two rates of star formation is retained as a free parameter; however, since the evolutionary models are relatively insensitive to this parameter, we have retained the value of 4.0 derived for the Galaxy by Dopita (1989).

The logic in favour of adopting a bimodal star formation rate is derived from several physical arguments. If CO-emitting molecular clouds map out the Galactic star forming regions, then their distribution offers strong support for star formation occurring at two quite different sites. The $\mathrm{CO}$ clouds are clearly divided into two populations which reflect their kinetic temperatures. The warm core $(\sim 100 \mathrm{~K})$, giant molecular clouds trace the spiral structure of the galaxy (Güsten and Mezger 1983) 
and are associated with $\mathrm{H}$ II regions, whereas the cold core $(\sim 5-15 \mathrm{~K})$ clouds are distributed smoothly throughout the Galaxy (Scoville, Sanders and Clemens 1986; Scoville and Good 1987).

In the warm core clouds it is believed that the higher mass stars form preferentially since the critical mass for fragmentation depends strongly on the gas temperature (Larson 1985). The association of these clouds with spiral arms suggests that cloud-cloud collisions or coalescences provide a heating mechanism for the clouds, and the compression necessary to trigger massive star formation within them. Further evidence for this mode of star formation comes from starburst galaxies, where several analyses suggest that only high mass stars are being formed and that the low mass cutoff in the IMF is of order $3 M_{\odot}$ (Rieke et al. 1980, 1985; Olaffsson, Bergrall and Ekman 1984; Augarde and Lequeux 1985).

Although high mass star formation may dominate in the very earliest stages of development of a normal galaxy (as shown, for instance, in the models of Dopita $1987 b$ ), the bulk of star formation in mature galaxies is believed to take place in the cold core molecular clouds; forming stars at a steady rate determined by gravitational fragmentation, and the repressurisation of the ISM by the T-Tauri winds from low mass $\left(<1 M_{\odot}\right)$ stars as they form (Norman and Silk 1980; Franco 1983; Franco and Cox 1983). Support for this scenario comes from the scaling relationships observed for the cold core clouds, between the virial mass and the radius, $M_{\mathrm{vir}} \propto R^{2}$, and between the turbulent velocity and the radius, $\Delta v \propto R^{1 / 2}$ (Larson 1981). Chièze (1987) has shown that this is exactly what would be expected if the clouds were close to gravitational instability in a constant pressure environment.

The form of the low mass component is unimportant since it has no effect on the chemical evolution. The form adopted for the high mass component is: 


$$
\psi(M)=\mathrm{a} \cdot\left[1-\exp \left\{-\left(M-M_{l o w} / 2\right) / 2 M_{l o w}\right\}\right] \cdot M^{-p},
$$

where the lower mass cutoff $M_{\text {low }}$ is chosen to give a peak near $1 M_{\odot}$, and to truncate the IMF below $0.5 M_{\odot}$. At high masses this function assumes a simple power law, and it is the slope $p$ that is important for stellar nucleosynthesis. In any case, this parameter is all that can be measured with any degree of completeness in the Magellanic Clouds. In the solar neighborhood, the high mass slope of the IMF is fairly well determined by observation to lie in the range $1.5 \leq p \leq 2.0$ (Burki 1977; Scalo 1986; Larson 1986 and references therein), while for the Magellanic Clouds the slope is somewhat steeper (Humphreys and McElroy 1984). The derived model parameters are given in Table 1, as well as the observationally determined parameters from the literature and the model results for the Galaxy from Dopita (1989). The parameters listed in bold type represent those that we have chosen specifically to fit with our models.

The high mass SFR is determined by the momentum balance in cloud-cloud collisions (see Dopita 1987b), so that at any particular time $t$.

$$
\operatorname{SFR}(t)=b \cdot M_{\text {gas }} \cdot\left(M_{\mathrm{tot}}\right)^{1 / 2},
$$

where $M_{\text {gas }}$ is the surface mass of gas (in $M_{\odot} / \mathrm{pc}^{2}$ ), $M_{\text {tot }}$ is the total surface mass of the material that has already fallen in, and the constant $b$ is given by

$$
b=\left(\tau_{\operatorname{depl}}[1+c]\right)^{-1},
$$

where $\tau_{\mathrm{depl}}$ is the gas depletion timescale, which is defined as the instantaneous gas mass divided by the rate of high mass star formation, and $c$ is the ratio of the low mass to high mass star formation rates mentioned previously.

The total surface mass of the star forming region is assumed to be initially zero, and to have increased as a result of infalling, zero-metallicity gas according to:

$$
M_{\mathrm{tot}}=M_{\mathrm{T}}\left(1-\exp \left[-t / \tau_{\mathrm{inf}}\right]\right)
$$

where $M_{\mathrm{T}}$ is the total surface mass of the system (determined from Lequeux 1979; 1984), and $\tau_{\text {inf }}$ is the gas infall timescale. Although corrections have been made for 


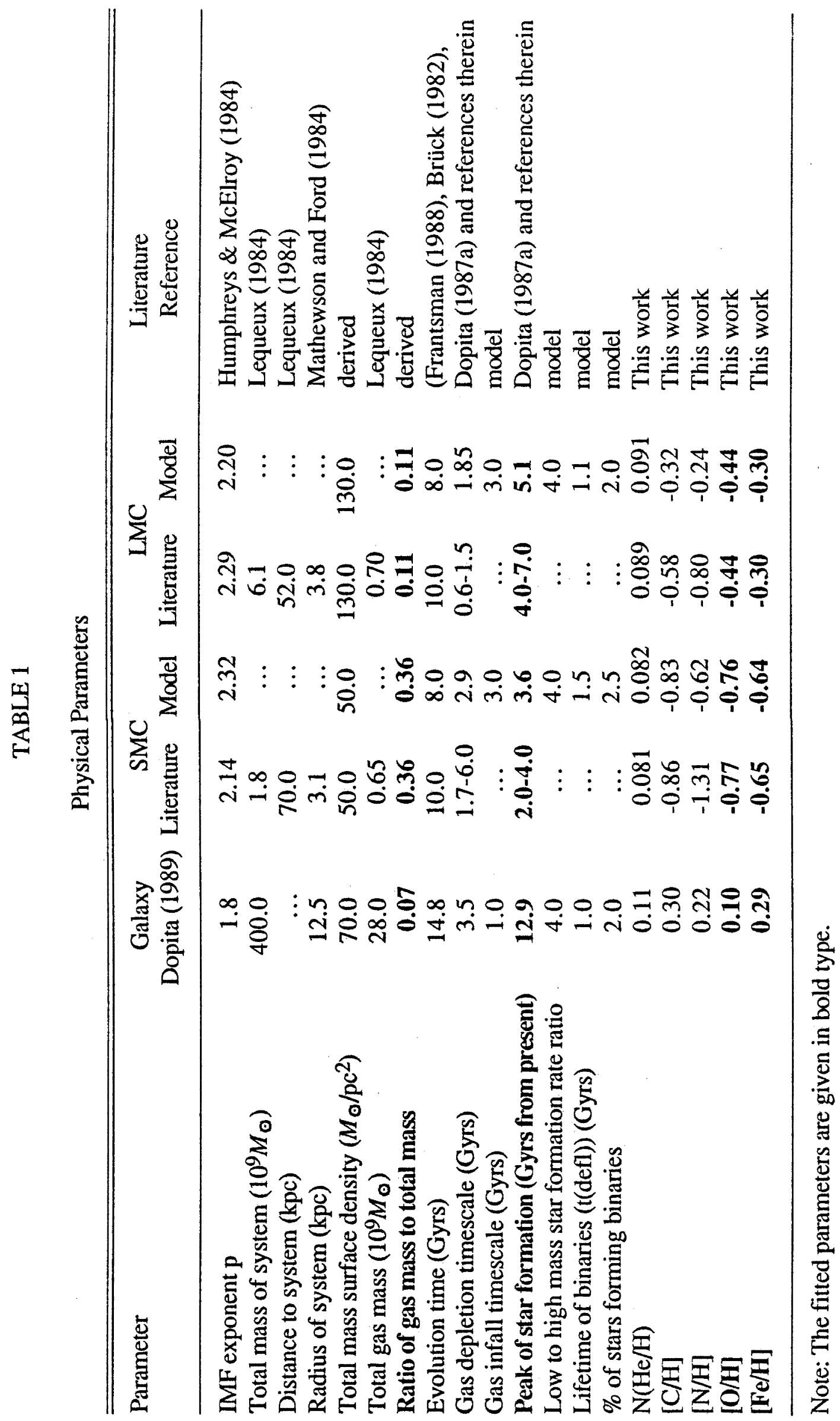


the helium content,the estimates of the gas surface densities given in Table 1 are probably lower bounds, since there is an unknown degree of saturation in the $21 \mathrm{~cm}$ line used in determining the $\mathrm{HI}$ surface density, and the contribution due to molecular hydrogen has been ignored. This last factor is probably not serious, however, since both dust and the $\mathrm{CO}$ surface density are observed to be low in the Clouds (see Israel 1984, for a full discussion).

The gas infall timescale is loosely constrained by the free-fall collapse timescale of the galaxy. This timescale, $\tau_{\mathrm{ff}}$, is given by

$$
\tau_{\mathrm{ff}}=1.65\left(R_{100} / M_{11}\right)^{1 / 2} \mathrm{Gyrs},
$$

where $R_{100}$ is the proto-galactic radius in units of $100 \mathrm{kpc}$, and $M_{11}$ is the galaxian mass in units of $10^{11} M_{\odot}$ (Dopita 1987c). For the Clouds this value is estimated to be between $\sim 2$ and 6 Gyrs. However there is the added constraint that the peak gas density occurs at the time of greatest star formation. This results from the strong observational correlation, noted by Dopita (1985), between the specific rate of star formation of massive stars, and the gas fraction, in the large sample of irregular and spiral galaxies observed by Donas and Deharveng (1984).

We avoid using the instantaneous recycling approximation since this is inaccurate for stars with lifetimes of the order of the gas depletion timescale. We therefore adopt the stellar lifetimes surgested by Iben and Renzini (1983):

$$
\begin{aligned}
\tau_{*} & =2.64 \times 10^{9} m^{-2.16} \mathrm{yr}, & & m>2.3 M_{\odot}, \\
\tau_{*} & =1.1 \times 10^{10} m^{-3.50} \mathrm{yr}, & & m<2.3 M_{\odot} .
\end{aligned}
$$

If the stellar lifetime is less than one time step $(0.15 \mathrm{Gyr})$ in computing the models, we assume that the stars die instantaneously and return the appropriate amount of heavy elements to the ISM.

The models were calculated over an assumed evolutionary time for each of the Clouds, that was initially taken to be 10 Gyrs, the age of the oldest known objects in each system (see references quoted in Table 1). However, more accurate models were obtained by assuming an age of $8 \mathrm{Gyrs}$ for the Clouds which probably better 
reflects the beginning of disk collapse in each Galaxy. Our models are therefore incapable of predicting anything older than 8 Gyrs., and on those grounds are possibly inaccurate (since, as shown in Table 1, the ages for some stars are estimated to be of the order of 10 Gyrs). Most likely, this is telling us that the star formation law for the Clouds is somewhat more complex than we have assumed, especially in the period of the slow collapse of the halo. Further modeling shows, however, that the absolute age of the system (within plausible bounds) does not seriously effect the major results derived from the models.

\section{b. The Nucleosynthesis Prescriptions}

The stars are divided up into three groups by mass, defined according to their ultimate fate as determined by current stellar evolution theory. The most massive stars $\left(M>12 M_{\odot}\right)$ die as Type II supernovae, and are the main source of heavy elements such as $\mathrm{O}, \mathrm{Ne}$, the $\alpha$-elements and the $r$-process elements (Arnett 1978, Woosley and Weaver 1982; 1986, Thielemann and Arnett 1985). The least massive stars are assumed to have an upper limit of $5 M_{\odot}$ (see for instance Tosi and Diaz 1985), which is "classically" thought to be the upper mass limit for stars incapable of igniting carbon in an electron degenerate core. These stars end their lives by ejection of their outer shells to form planetary nebulae, and leave behind a white dwarf. The nucleosynthesis prescription of Renzini and Voli (1981), with a mass loss parameter of $\eta=0.33$ and a mixing length parameter $\alpha=1.5$, has been used for this mass range. These stars contribute mainly ${ }^{4} \mathrm{He},{ }^{12} \mathrm{C},{ }^{13} \mathrm{C},{ }^{14} \mathrm{~N}$, and $s$ process elements to the enrichment of the ISM.

In the mass range from five to twelve solar masses, the fate of the stars is still controversial. Up to a mass of $\sim 8 M_{\odot}$, the stars are theoretically capable of building electron degenerate $\mathrm{C}-\mathrm{O}$ cores having masses up to the Chandrasekhar limit (see Nomoto, Thielemann, and Yokoi 1984; Thielemann, Nomoto, Yokoi 1986), in which case they would explode by detonation or carbon deflagration. Both 
scenarios would result in total disruption of the star, however the former is rendered less likely due to the large overabundance of $\mathrm{Fe}$ expected if they occur in appreciable numbers (see Audouze, Chiosi and Woosley 1986). The carbon deflagration supernovae would produce a range of possible nucleosynthetic prescriptions depending on the velocity of the deflagration wave, and would by and large mimic the effects of the deflagration of binary C-O white dwarfs (discussed further on). With the inclusion of convective overshooting in stellar model calculations, however, the mass range where single star deflagration is possible may be reduced to insignificant levels (Matteucci and Tosi 1986, Castellani et al. 1985, Renzini et al 1985), and it may be that stars of 6 to $8 M_{\odot}$ actually produce Type II supernovae as suggested by Tornambè and Matteucci (1985).

The fate of stars in the mass range 8 to $12 M_{\odot}$ has been reviewed by Nomoto 1984. They are thought to collapse due to electron captures in the core, and if the explosions resulting from this do not totally disrupt the star, the ejecta probably make no significant contribution to the enrichment of the ISM owing to the small amount of heavy element enriched matter overlying the degenerate $\mathrm{O} / \mathrm{Ne} / \mathrm{Mg}$ core.

Owing to the present uncertainties involved in modeling the whole mass range from 5 to $12 M_{\odot}$, we assume for our models the nucleosynthetic prescriptions of Renzini and Voli (1981) for $5<M / M_{\odot}<8$ whereby these stars end their lives by ejecting only their outer envelope, leaving behind a white dwarf. The contributions from the mass range $8<M / M_{\odot}<12$ are assumed to be negligible.

The mass (in solar masses) of the remnants, mrem, assumed to be left behind after the death of a single star of any mass is based on the work of Iben and Tutukov (1985) and Amett (1978):

$$
\begin{aligned}
m_{\text {rem }} & =0.35+0.22 m & & m<5 M_{\odot}, \\
& =1.42 & & 5 \mathrm{M}_{\odot}<m<12 M_{\odot}, \\
& =1.42+0.01(m-11.5) & & m>12 M_{\odot} .
\end{aligned}
$$


All stars in the mass range $3<M / M_{\odot}<12$ may, however, still end their lives as supernovae, if they form part of a binary system. The most successful models for the progenitors of Type Ia supernovae assume that they originate from the carbon deflagration of C-O white dwarfs in a binary system (Whelan and Iben 1973, Iben and Tutukov $1984 ; 1985)$. These would result in the total disruption of the star and the ejection of large amounts of Fe, and lesser, but still significant, amounts of the elements from $\mathrm{Si}$ to $\mathrm{Ca}$ (see Nomoto, Thielemann, and Yokoi 1984, case C6, and Thielemann, Nomoto, and Yokoi 1986).

Type Ib supernovae could also result from C-O white dwarfs in binary systems, in this case by an off center He-detonation (Branch and Nomoto, 1986; Iben et al. 1987; Tornambé and Matteucci 1987), although there is some suggestion that these supernovae arise from the explosion of Wolf-Rayet stars (Gaskell et al. 1986; Filippenko and Sargent 1986). This type of supernova is, however, relatively rare, forming just 10\% of all Type I supernovae (Audouze, Chiosi and Woosley 1986), and at this level of accuracy can easily be ignored.

Owing to the difficulty in modeling the exact range of semi-major axes that might occur in C-O binary pairs, and hence the delay before they would coalesce, we simplify the problem by assuming the Type I supernova precursors to be an exponentially decaying fraction $(\sim 1-4 \%)$ of all the white dwarfs which are formed. The actual percentage is influenced by the infall timescale and the deflagration timescale (characteristic of the period between the formation of the white dwarf, and its subsequent deflagration). Satisfactory models for the Galaxy were produced by Dopita (1989) supposing this fraction to be $2 \%$. Although this figure is not well constrained, it is in agreement with the expected number of Type I supernovae for the Galaxy (van den Bergh, McClure, and Evans 1987), which also results in 2\% (with an estimated error of a factor of two) of the expected number of white dwarfs (if we assume each white dwarf to be $1.4 M_{\odot}$ ). 


\section{c. The chemical evolution}

We have specifically modelled the abundance variations of $\mathrm{He}, \mathrm{C}, \mathrm{N}, \mathrm{O}$, and Fe. These elements encompass the three main contributions to nucleosynthesis of the elements: $\mathrm{C}$ and $\mathrm{N}$ are produced in the low to intermediate mass range, $\mathrm{O}$ is produced in massive stars ( $\left.\geq 12 M_{\odot}\right)$, and $\mathrm{Fe}$ is produced mainly from Type Ia supernovae, but with a significant contribution $(\sim 1 / 3)$ from Type II supernovae. Included in the $\mathrm{Fe}$-peak elements would be $\mathrm{Cr}$ and $\mathrm{Ni}$ (and to some extent $\mathrm{Zn}$ ), whereas the $\alpha$-elements, $\mathrm{Ne}, \mathrm{Mg}, \mathrm{S}, \mathrm{Si}, \mathrm{Ca}$ and $\mathrm{Ti}$ follow the evolution of $\mathrm{O}$.

The low-abundance asymptotic value for the $[\mathrm{O} / \mathrm{Fe}]$ ratio (where we use the usual notation $\left.[M / X]=\log _{10} N(M / X)_{\text {object }}-\log _{10} N(M / X)_{\odot}\right)$ puts strong constraints on the production of iron in normal Type II supernovae (since the lower mass precursors of Type Ia supernovae have not had a chance to evolve). As discussed by Dopita (1989), the explosion of SN 1987A allows us to estimate the value of this ratio. He found that this supernova produced a ratio of $[\mathrm{O} / \mathrm{Fe}]=$ $0.65 \pm 0.25$, which is very similar to the value observed in extreme Population II halo stars in the Galaxy; $[\mathrm{O} / \mathrm{Fe}]=0.5 \pm 0.2$ (Sneden, 1985; Wheeler, Sneden, and Truran 1989). This suggests that such Type II supernovae may have dominated the nucleosynthesis during the collapse phase of our Galaxy.

The evolution of $[\mathrm{O} / \mathrm{Fe}]$ with metallicity is driven by the slope of the IMF and by the relevant timescales; the timescale for infall of halo gas, and the deflagration timescale. The deflagration timescale ( $\left.\tau_{\text {defl }}\right)$ depends critically (to the fourth power) on the semi-major axis of the precursor binary system (Iben and Tutukov 1984; Matteucci and Greggio 1986). Models of the Galaxy by Dopita (1989) indicate that the observed abundance ratios of the $\alpha$-elements relative to $\mathrm{Fe}$ as a function of [Fe/H] (see Fig. 1), are best fitted assuming a deflagration timescale of the order of 1.0 $\pm 0.5 \mathrm{Gyr}$. It may be seen from Figure 1 that the consequence of assuming a deflagration timescale which is too long is that the delay before Fe enrichment from Type I supernovae starts to take effect is overestimated and the slope of the increase 
Figure 1 An illustration, from Dopita (1989), of the fits of several models with different deflagration timescales $t$ (defl), to the collected Galactic data on the [ $\alpha$ element/Fe] ratio plotted against $[\mathrm{Fe} / \mathrm{H}]$ (from Wheeler, Sneden, and Truran). 


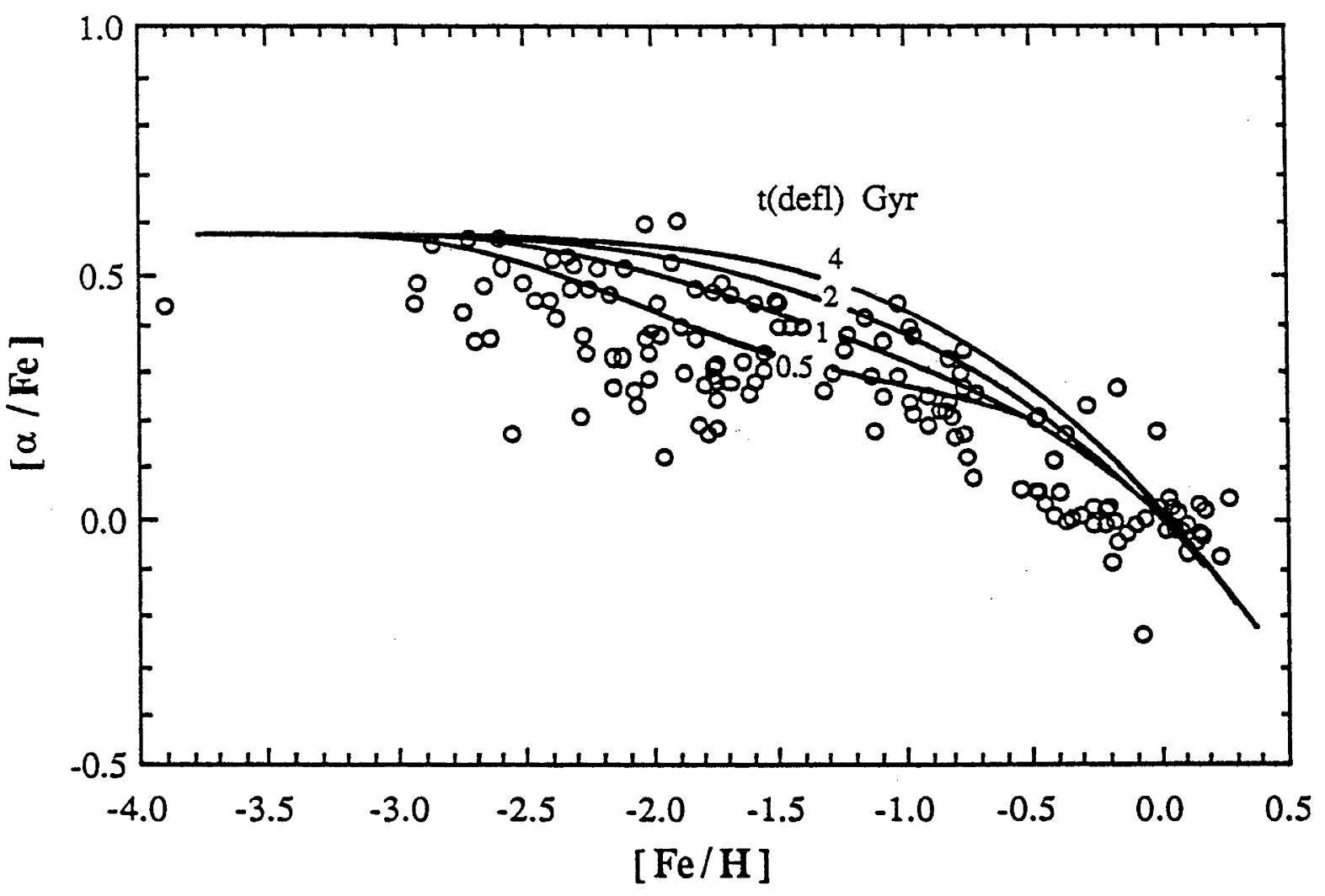


in the $[\alpha / \mathrm{Fe}]$ ratio becomes too steep. At times long compared to $\tau_{\mathrm{defl}}$, however, the $[\alpha / \mathrm{Fe}]$ ratio asymptotes to a common slope for all values of the timescale.

\title{
III. THE RELATIVE ABUNDANCE DISTRIBUTION OF THE ELEMENTS
}

\author{
a. Carbon, nitrogen, and oxygen
}

In Figures $2 a$ to $2 c$ we plot against $[\mathrm{Fe} / \mathrm{H}]$ the model results for the $[\mathrm{C} / \mathrm{Fe}]$, $[\mathrm{N} / \mathrm{Fe}]$, and $[\mathrm{O} / \mathrm{Fe}]$ ratios respectively. We have included in these diagrams data derived from papers I and II (relative to the local Galactic ISM), and several sources from the literature. The filled circles represent data from the SMC, and the open circles are from the LMC. Due to the incomplete coverage of the full range of elements observable in any one object, different objects are represented in each figure. It may be seen from these graphs that, at the present levels of accuracy, we may consider the objects in both of the Clouds to form a continuous abundance series without introducing much additional error.

In Figure $2 a$ we have plotted the $[\mathrm{C} / \mathrm{Fe}]$ ratios for the $\mathrm{F}$-supergiants we studied in paper I, the F-supergiants of Spite, Barbuy, and Spite (1989), and the Bstars (A3 and A12) from Reiterman et al. (1988). For these diagrams, and those that follow, we have included an error bar of \pm 0.2 dex to give an indication of the scale of the most probable experimental error.

The models are in excellent agreement with the data to well within the experimental errors, although the only SNR that is included in this figure (N49 as marked), appears to be somewhat overdeficient in $\mathrm{C}$ (relative to $\mathrm{Fe}$ ). This may well be due to survival of graphite grains in the recombination region of the SNR. Further information on the $\mathrm{C}$ abundances may be gained from Figure $3 a$, which shows the model results for $[\mathrm{C} / \mathrm{O}]$ versus $[\mathrm{O} / \mathrm{H}]$, and all the available observational 
Figure 2 A plot of the (a) $[\mathrm{C} / \mathrm{Fe}]$, (b) $[\mathrm{N} / \mathrm{Fe}]$, and (c) $[\mathrm{O} / \mathrm{Fe}]$ ratios against $[\mathrm{Fe} / \mathrm{H}]$ for the Galactic model (dashed line), and the Magellanic Cloud models (solid lines as marked). The data derived from papers I and II are plotted here as open circles for the LMC, and closed circles for the SMC. An error bar of \pm 0.2 dex is included to remind the reader of the scale, and the typical sort of error to expect in the data. 

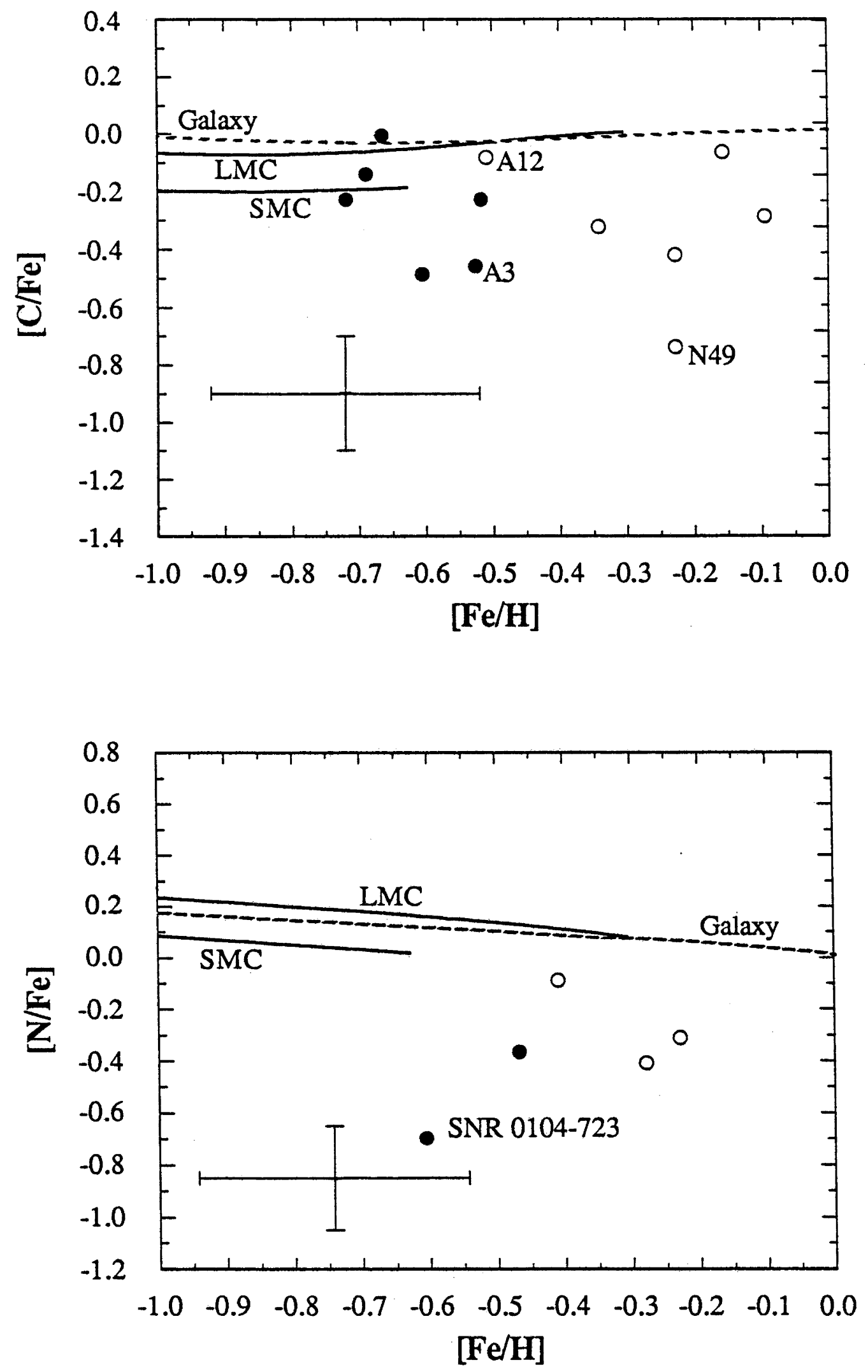


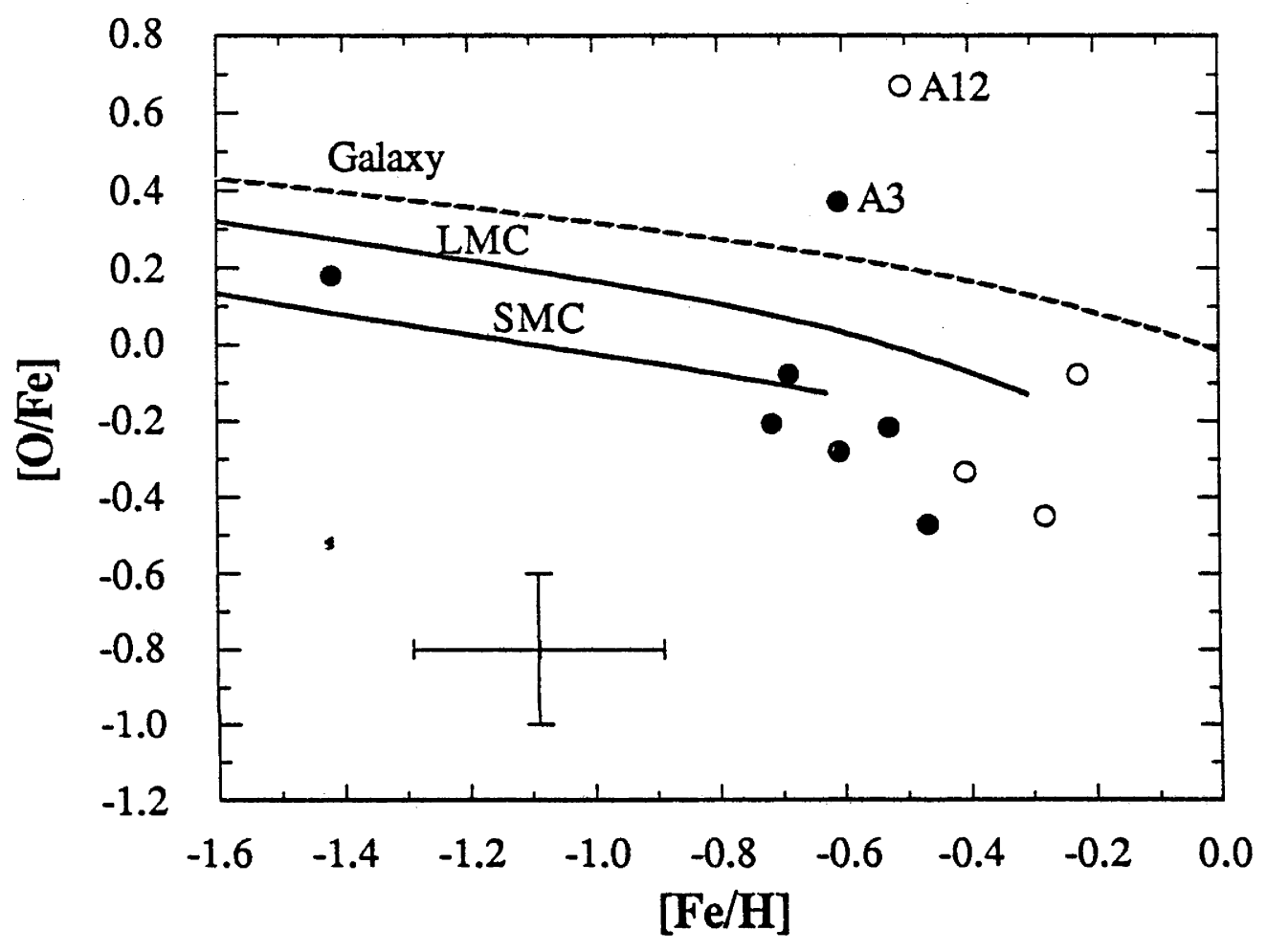


Figure 3 A plot of the (a) $[\mathrm{C} / \mathrm{O}]$, and (b) $[\mathrm{N} / \mathrm{O}]$ ratios against $[\mathrm{O} / \mathrm{H}]$ for the Galactic model (dashed line), and the Magellanic Cloud models (solid lines as marked). The data derived from papers I and II are plotted here as open circles for the LMC, and closed circles for the SMC. An error bar of $\pm 0.2 \mathrm{dex}$ is included to remind the reader of the scale, and the typical sort of error to expect in the data. 

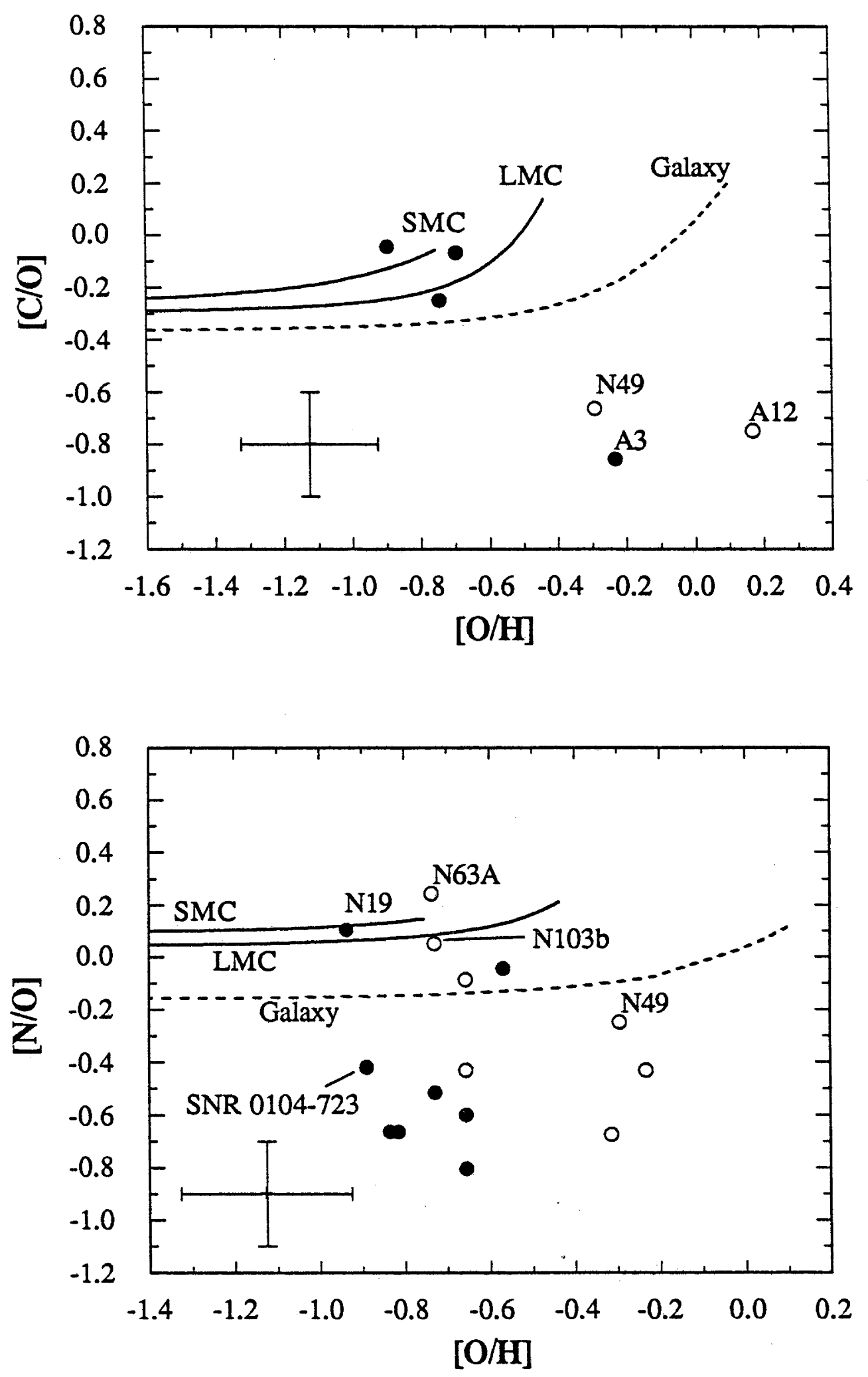
data for these quantities in the Magellanic Clouds. Again we see that our models provide an excellent fit to these data. The supernova remnant $\mathrm{N} 49$ is again observed to be overdeficient in $\mathrm{C}$ (relative to $\mathrm{O}$ ), which suggests that it is indeed the carbon that is low, rather than both $\mathrm{Fe}$ and $\mathrm{O}$ that are high. Conversely, the two B-stars studied by Reiterman et al. (1988) are observed to have a normal C abundance relative to $\mathrm{Fe}$ in Figure $2 a$, but a large underabundance of $\mathrm{C}$ relative to $\mathrm{O}$ in Figure 3a. This clearly indicates that it is the $\mathrm{O}$ abundance in these stars which is anomalously high. It will be interesting to see if further analysis of these stars, and others like them, substantiate the high $\mathrm{O}$ abundances.

In conclusion, the excellent fit of our models to the $\mathrm{C}$ abundances (not specifically fitted in our derivation of the models) of the F-supergiants, expresses support for our models, but more importantly, we believe that we have at last answered the question of why the $C$ abundances in the Magellanic Clouds are apparently so very low (from the analysis of UV spectra of $\mathrm{H} I I$ regions by Dufour, Shields, and Talbot 1982, and Dufour, Schiffer, and Shields 1984). The answer seems to be that the $\mathrm{C}$ abundances are entirely normal in the Magellanic Clouds, and it is only the $\mathrm{H} \mathrm{II}$ regions that are reflecting severe under-abundances of $\mathrm{C}$. These under-abundances then, are intrinsic to the $\mathrm{H} \mathrm{II}$ regions, and an explanation for this must be sought in terms of such processes as graphite grain formation, with particle sizes perhaps somewhat larger than is common in our own Galaxy in order to simultaneously account for the weakness of the $2200 \AA$ absorption feature observed in the ISM of the Magellanic Clouds (see Koorneef and Code 1981, Bromage and Nandy 1983, Fitzpatrick and Savage 1984, Nandy, Morgan, and Houziaux 1984).

In Figure $2 b$ we plot $[\mathrm{N} / \mathrm{Fe}]$ versus the $[\mathrm{Fe} / \mathrm{H}]$ ratio for our models, and the data derived solely from the SNRs we studied in paper II. Considering the severe underabundance of $\mathrm{N}$ found in most $\mathrm{H}$ II regions, our models fit all the SNR data, except SNR 0104-723, surprisingly well. The scatter increases somewhat when we turn to Figure $3 b$, where we plot the $[\mathrm{N} / \mathrm{O}]$ ratio versus $[\mathrm{O} / \mathrm{H}]$ for both the SNRs (as 
marked) and the $\mathrm{H}$ II regions from paper II. This is reminiscent of the scatter found in [N/O] for dwarf irregular galaxies (Dufour 1986, Matteucci and Tosi 1985), Blue Compact Galaxies (see Pagel and Edmunds 1981, and references therein), and Galactic H II regions (Pagel 1985). However, it is still evident from Figure $3 b$ that the SNRs are systematically more enhanced in $\mathrm{N}$ than are the $\mathrm{H}$ II regions. This may well be a modeling problem, but if so, the cause remains obscure. More interestingly, we may be seeing a genuine difference between the $\mathrm{N}$ abundances in these types of object. Either some process is depleting the $\mathrm{N}$ abundances observed in $\mathrm{H}$ II regions, or the SNR abundances are enhanced. Evidence for $\mathrm{N}$ enhancements in the atmospheres of massive stars is widespread (Luck and Lambert 1981), and if ejected as a stellar wind, this material could pollute the surrounding ISM. Evidence that this may indeed happen comes from observations of nitrogen rich knots in several SNRs, most notably Puppis A, and the observation of highly nitrogen enriched ejecta around SN 1987A (Kirshner 1988, Fransson et al. 1989).

Many suggestions have been put forward, in recent years, to explain the observed spread in [N/O]. Matteucci and Chiosi (1983), for instance suggested that the spread in $\mathrm{N}$ and $\mathrm{O}$ abundances with the fractional gas mass, $M_{\mathrm{gas}} / M_{\text {tot }}$ might be due to variations, from galaxy to galaxy, in:

i) the IMF and/or the chemical yields,

ii) the infall rate/SFR ratio,

iii) the rate of mass outflow/SFR ratio.

While Matteucci and Tosi (1985) suggest that the spread may be explained by a variation in the galactic wind/SFR ratio from galaxy to galaxy.

Similarly, many authors (for example Alloin et al. 1979, Pagel and Edmunds 1981, Serrano and Peimbert 1983) have tried to explain the spread in the [N/O] ratio in terms of differing contributions of primary and secondary nitrogen. Matteucci and Tosi (1985) suggest that it is due to variations in the ambient metallicity affecting the limiting mass, $\mathrm{M}_{\mathrm{up}}$, required for accumulating degenerate carbon cores and thereby 
affecting the mass range of stars capable of producing primary nitrogen. In addition Tosi (1988) showed that $\mathrm{N}$ is strongly reduced in abundance when the effects of overshooting are included due to the reduction in the stellar envelopes as the core size is increased, and to the decrease in the mass range of stars experiencing envelope burning (see Greggio and Tosi 1986). However, other uncertainties, like the rate of the ${ }^{12} \mathrm{C}(\alpha, \gamma){ }^{16} \mathrm{O}$ reaction (we have assumed the standard rate throughout), could also have a significant influence on how the $\mathrm{N}$ abundance might vary from one environment to another (see Matteucci 1986 for a discussion of the consequences of adopting the higher rate put forward by Kettner et al. 1982).

In conclusion, the production mechanisms for nitrogen are still highly controversial, and until they are solved satisfactorily, nitrogen will remain a poor diagnostic for determining the star formation histories of galaxies.

In Figure $2 c$ we show our model results for the [O/Fe] ratio plotted against $[\mathrm{Fe} / \mathrm{H}]$, superimposed on the observational data available to us. These data are derived from all of the SNRs we studied in paper II, the three F-supergiants and one red supergiant studied by Spite, Spite, and François (1989) and Spite et al. (1986) respectively, and the two B-stars studied by Reitermann et al. (1988). Again the Bstars are overabundant in oxygen, but our models fit all the other data remarkably well, hence adding support to the validity of the models (the models having been forced to fit the $\mathrm{H} \mathrm{II}$ region $\mathrm{O}$ abundances, which could not be displayed in this figure).

\section{b. The $\alpha$-elements}

The $\alpha$-elements are defined as those which have been built by $\alpha$-particle addition to seed nuclei, and therefore have an even number of protons and neutrons in the nuclei of their major isotope. Most workers agree that the elements $\mathrm{O}, \mathrm{Mg}, \mathrm{Si}$, $\mathrm{S}$, and $\mathrm{Ca}$ belong to this group, but Ti has sometimes been excluded. Certainly a part of the Ti abundance is contributed through the $\alpha$-process, whilst the rest 
probably results from the equilibrium burning to the iron-peak elements. However the $[\mathrm{Ti} / \mathrm{Fe}]$ ratio appears to act like an $\alpha$-element in its variation with $\mathrm{Fe}$ abundance (see Magain 1989), so the contribution from the equilibrium process must be quite small. On this basis, $\mathrm{Ti}$ is included here as a member.

Since the $\alpha$-elements are produced by Type II supernovae of massive stars, whilst the bulk of $\mathrm{Fe}$ is derived from deflagration of $\mathrm{C}-\mathrm{O}$ white dwarfs on a much longer timescale, the ratio of [ $\alpha$-element/Fe] puts interesting constraints on the past history of low mass to high mass star formation.

In Figure 4 we plot the combined contributions of the three well observed $\alpha$ elements in our data according to the formula:

\section{$1 / 3([\mathrm{Mg} / \mathrm{Fe}]+[\mathrm{Ca} / \mathrm{Fe}]+[\mathrm{Ti} / \mathrm{Fe}])$}

where at least two elements have been used in determining the average, and when one is missing, the mean of the average differences between that element and the other two is maintained in the substitute. In addition, we have plotted our model results for the $[\mathrm{O} / \mathrm{Fe}]$ ratio, which, as discussed previously, follows the trends of the other $\alpha$-elements closely. We see that the models fit the data within the experimental errors, although there may be an indication that they underestimate the $\alpha$-abundances by $0.1-0.2$ dex. Since the models have been fitted to the $O$ abundances, this would indicate that the other $\alpha$-elements are overabundant relative to $\mathrm{O}$ in the Clouds, which is perhaps the result of the different stellar mass ranges responsible for the bulk of each individual element. Indeed, variations of this order between different $\alpha$-elements is a feature of the Galactic abundances in this metallicity range (see Andersen et al. 1988).

The most obvious feature apparent in both Figures $2 c$ and 4 is the systematically lower [alpha/Fe] ratio observed in the Clouds relative to the Galaxy. As discussed in §II, the evolution of this ratio with metallicity is driven by the slope of the IMF, the infall timescale and the deflagration timescale. As may be seen from Table 1, our results indicate that it is not the latter timescale that could be responsible 
Figure 4 A plot of the [alpha-elements $/ \mathrm{Fe}]$ ratio against $[\mathrm{Fe} / \mathrm{H}]$ for the Galactic model (dashed line), and the Magellanic Cloud models (solid lines as marked). The data derived from papers I and II are plotted here as open circles for the LMC, and closed circles for the SMC. The model $\alpha$-elements are represented by oxygen, whilst the observational data is derived from the sum of several $\alpha$ elements (see text). An error bar of \pm 0.2 dex is included to remind the reader of the scale, and the typical sort of error to expect in the data. 


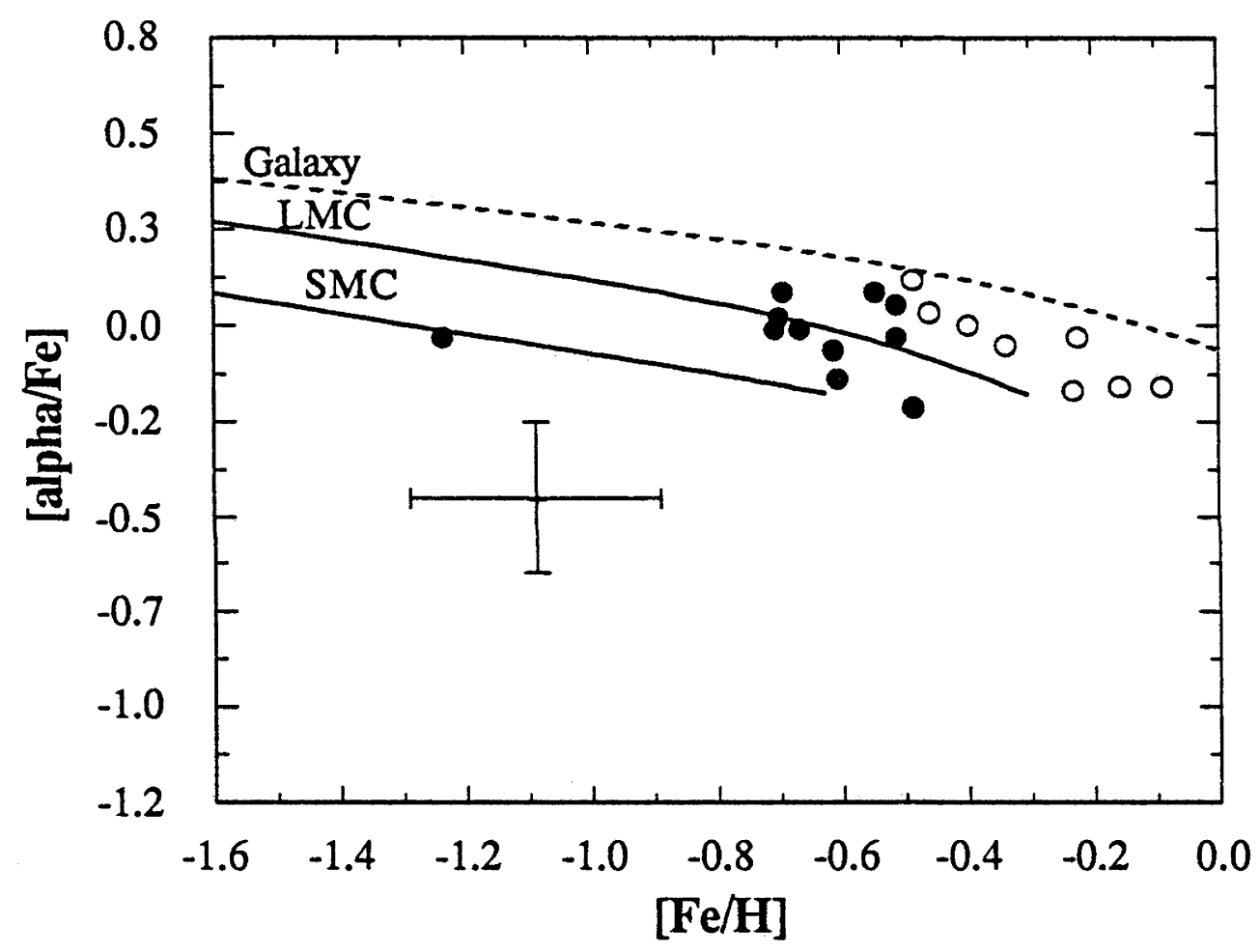


for the lower ratio in the Clouds, since it is longer in the Clouds than in the Galaxy, which would give a lower $\mathrm{Fe}$ abundance and thus a higher [alpha/Fe] ratio. The infall timescale for each of the Clouds is also substantially longer than for the Galaxy, again having a result in the opposite sense to the one observed. It is therefore the steeper slope of the IMF that is the critical factor in reducing the [alpha/Fe] ratio, through the relative decrease in the numbers of massive stars.

\section{c. Neon and Argon}

The two remaining even elements in the mass range encompassed by the $\alpha$ elements, $\mathrm{Ne}$ and $\mathrm{Ar}$, are also produced in massive stars. Neon is produced mainly during hydrostatic C-burning (Audouze, Chiosi, and Woosley 1986), and is therefore sensitive to the accepted ${ }^{12} \mathrm{C}(\alpha, \gamma)^{16} \mathrm{O}$ reaction rate, whilst the bulk of $\mathrm{Ar}$ is produced during explosive oxygen and silicon burning, and is thus sensitive to the slope of the IMF. Nevertheless, both of these elements are expected to scale with the $\alpha$-elements, and this is born out in case of the the Magellanic Clouds as shown in Figures $5 a$ and $5 b$.

Our data show no sign of variation in $[\mathrm{Ne} / \mathrm{O}]$ or $[\mathrm{Ar} / \mathrm{O}]$ ratios with decreasing $[\mathrm{O} / \mathrm{H}]$, although the scatter in both is quite large $( \pm 0.30$ and \pm 0.34 dex respectively). Both $\mathrm{Ne}$ and $\mathrm{Ar}$ appear to be somewhat overabundant relative to $\mathrm{O}$ ( 0.36 and 0.09 dex respectively), however this result may not be significant. In the case of Ar, the overabundance results from the SNR data alone, and is likely to be due to modeling errors, since only minor ionization species were observed. A possible solution suggested in paper II, was to attempt observations of the prominent ArII] line at $6.985 \mu \mathrm{m}$ in order to gain information on one of the more abundant ionization species. Similarly, the Ne abundance in both $\mathrm{H}$ II regions and SNRs depends on observation of only one ionization species, [NeIII]. It was suggested in paper II that observations of the UV lines of [NeIV] and [NeV] would be a valuable check on the abundances derived from [NeIII] alone. 
Figure 5 A plot of (a) the $[\mathrm{Ne} / \mathrm{O}]$ ratio, and (b) the $[\mathrm{Ar} / \mathrm{O}]$ ratio, versus $[\mathrm{O} / \mathrm{H}]$ for the LMC (open circles), and the SMC (closed circles). As for the the previous figures, a 0.2 dex error bar has been included as an indication of the errors and the scale. 

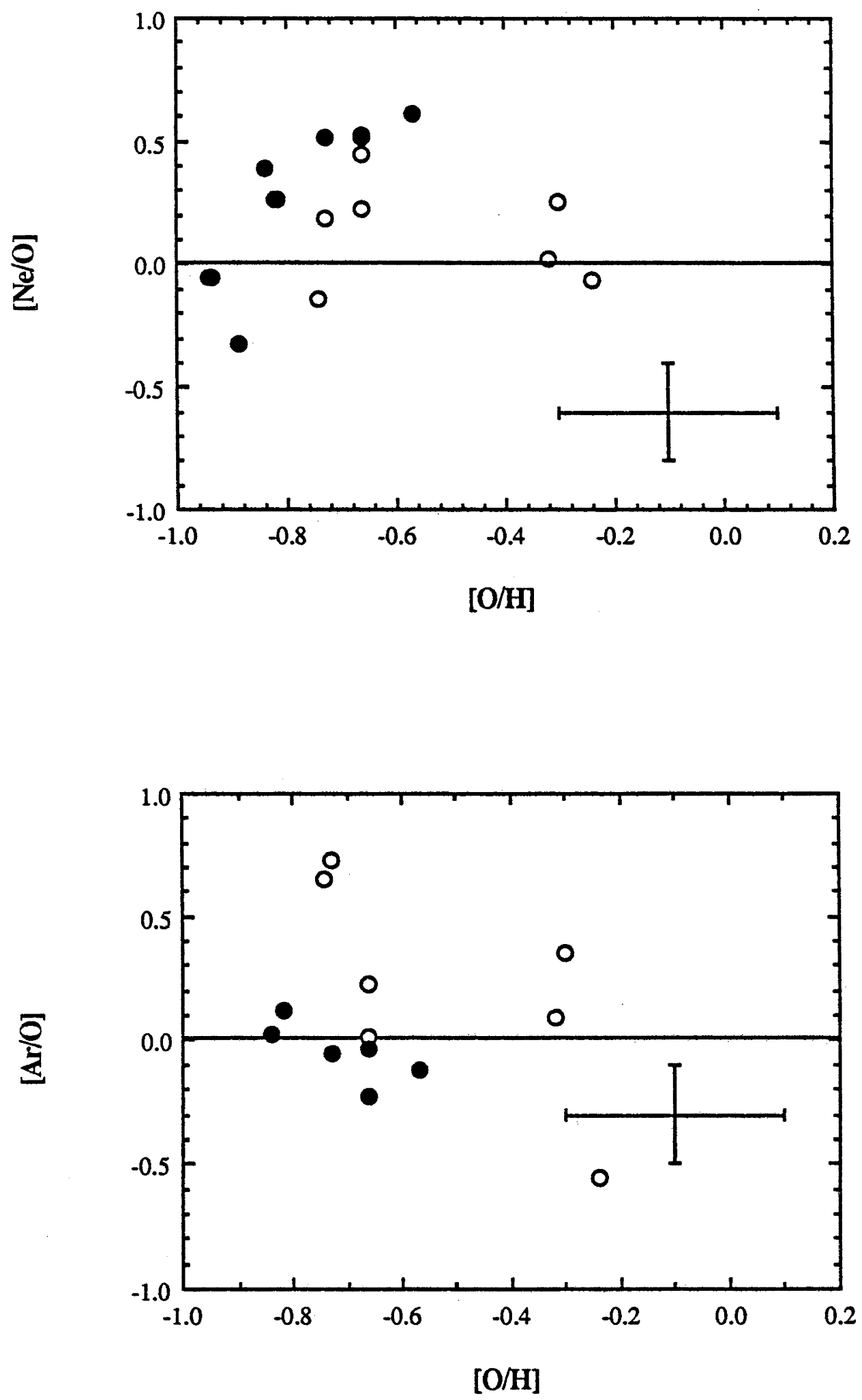


\section{d. The odd elements}

We include in this section the odd elements $\mathrm{Na}$ and $\mathrm{Al}$. These lie below those of the Fe-peak group in atomic mass, and we have little information on their abundances at present. For $\mathrm{Na}$, the combined analyses of our own work (paper I), Spite et al. (1986), and Spite, Spite, and François (1989) give us information on a total of six stars, which is only sufficient to show that their abundances relative to Fe are no different to that of the solar vicinity, within the experimental errors. For Al the situation is worse since there is data for only one star (from Spite, Spite, and François 1989).

The odd elements of the Fe-peak comprise Sc, $\mathrm{V}, \mathrm{Mn}, \mathrm{Co}$, and $\mathrm{Cu}$, and they owe their existence to neutron irradiation of seed nuclei during the major stellar burning phases. The abundances are therefore highly dependent on the neutron flux and the interior temperatures of stars, as well as the abundances of the seed nuclei. As such, these elements have the potential for acting as sensitive probes of the temperatures of stellar interiors. Unfortunately, the fact that all these elements have unpaired protons means that they all experience hyperfine splitting ( $h f s$ ) of their energy levels due to the presence of a nuclear magnetic moment. Little effort has been put into correcting for the $h f s$ in the past (except for the case of $\mathrm{Mn}$ which has been studied carefully by Beynon 1978a,b, and Gratton 1989) so it is still uncertain how the abundances vary in our own Galaxy. Instead of using precise oscillator strengths (often not available for the transition of interest anyway) most workers have relied on empirical corrections to the turbulent velocity $\xi_{\text {Turb }}$ in deriving the abundances. This is clearly unsatisfactory, as shown by the large scatter in abundances derived for stars from different sources (Lambert 1989, and Wheeler, Sneden, and Truran 1989). In Paper I, we also used an empirical technique to estimate the extra $\xi_{\text {Turb }}$ required to correct for the $h f s$, based on some careful work by Gratton (1982) and the Liege solar atlas. However, since we measured our 
abundances differentially with respect to Canopus, any errors in the hfs correction should cancel out.

In Figures $6 a$ to $6 c$ we plot [Sc/Ti], [V/Cr], and [Mn/Fe] respectively, against the $\mathrm{Fe}$ abundance $[\mathrm{Fe} / \mathrm{H}]$. That is, we plot the ratios of the odd-elements, with their nearest neighboring even-elements, to see if there is any sign of an odd-even effect. We see immediately that there is no obvious effect for Sc or $\mathrm{Mn}$, while $\mathrm{V}$ is apparently overabundant.

In the field stars of the Galaxy, Sc and V are believed to be invariant relative to Fe with decreasing metallicity (Wheeler, Sneden, and Truran 1989), however the lack of accurate $h f s$ corrections weakens these conclusions. The globular clusters,

on the other hand, display a systematic overabundance of $\sim 0.25 \mathrm{dex}$ in [V/Fe] (Wheeler, Sneden, and Truran 1989 and references therein). This may be an indication that the process acting to enhance the $\mathrm{V}$ abundance in the Galactic globular clusters is also of importance in the Magellanic Clouds.

The $\mathrm{Mn}$ abundances in the Galaxy shows some sign of falling for $[\mathrm{Fe} / \mathrm{H}]<\sim-$ 0.4. Considering the scatter in our abundances, however, we cannot rule out a similar drop in the Mn abundances for the Magellanic Clouds.

We may conclude that our observations for all three elements are in substantial agreement with the Galactic results, except that $V$ maybe somewhat overproduced relative to the field stars.

\section{e. The even Fe-peak elements}

The only two elements that we consider in this category, besides Fe itself, are $\mathrm{Cr}$ and $\mathrm{Ni}$. The relative abundances of these elements are plotted against $[\mathrm{Fe} / \mathrm{H}]$ in Figures $7 a$ and $7 b$, where it is evident that both elements deviate little from proportional production with iron. Most of the evidence in the literature agrees that this is also the case for the Galaxy, and since these elements, together with Fe, are formed in the equilibrium process during supernova explosions of both Type I and 
Figure 6 The odd-even closest neighbours (a) [Sc/Ti], (b) [V/Cr], and (c) $[\mathrm{Mn} / \mathrm{Fe}]$, plotted against $[\mathrm{Fe} / \mathrm{H}]$, for the LMC (open circles), and the SMC (closed circles). As for the the previous figures, a 0.2 dex error bar has been included as an indication of the errors and the scale. 

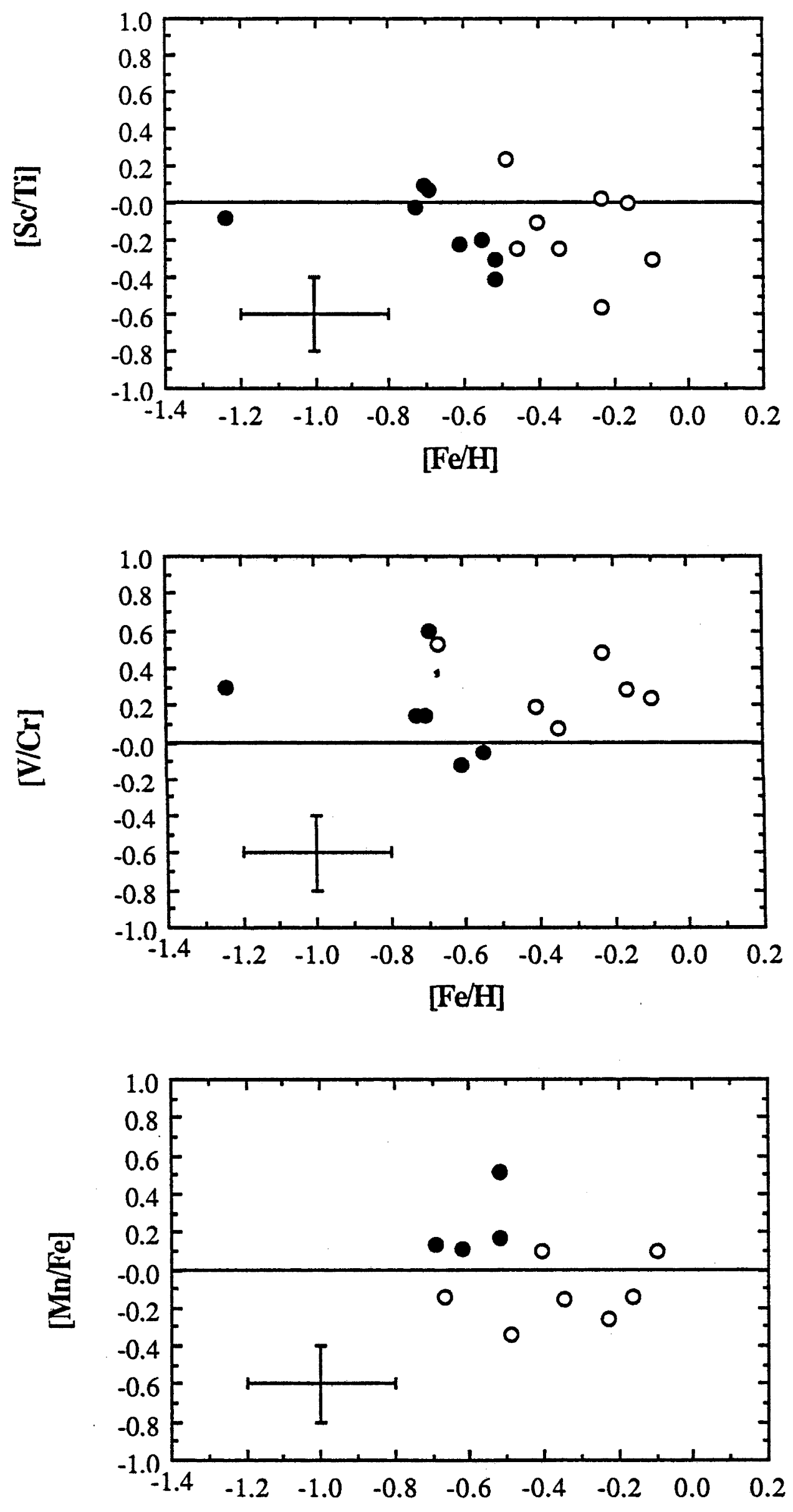

[Fe/H] 
Figure 7 The even iron-peak element ratios (a) $[\mathrm{Cr} / \mathrm{Fe}]$, and (b) $[\mathrm{Ni} / \mathrm{Fe}]$, plotted against $[\mathrm{Fe} / \mathrm{H}]$. The LMC (open circles), and the SMC (closed circles) are plotted, and, as for the the previous figures, a 0.2 dex error bar has been included as an indication of the errors and the scale. 

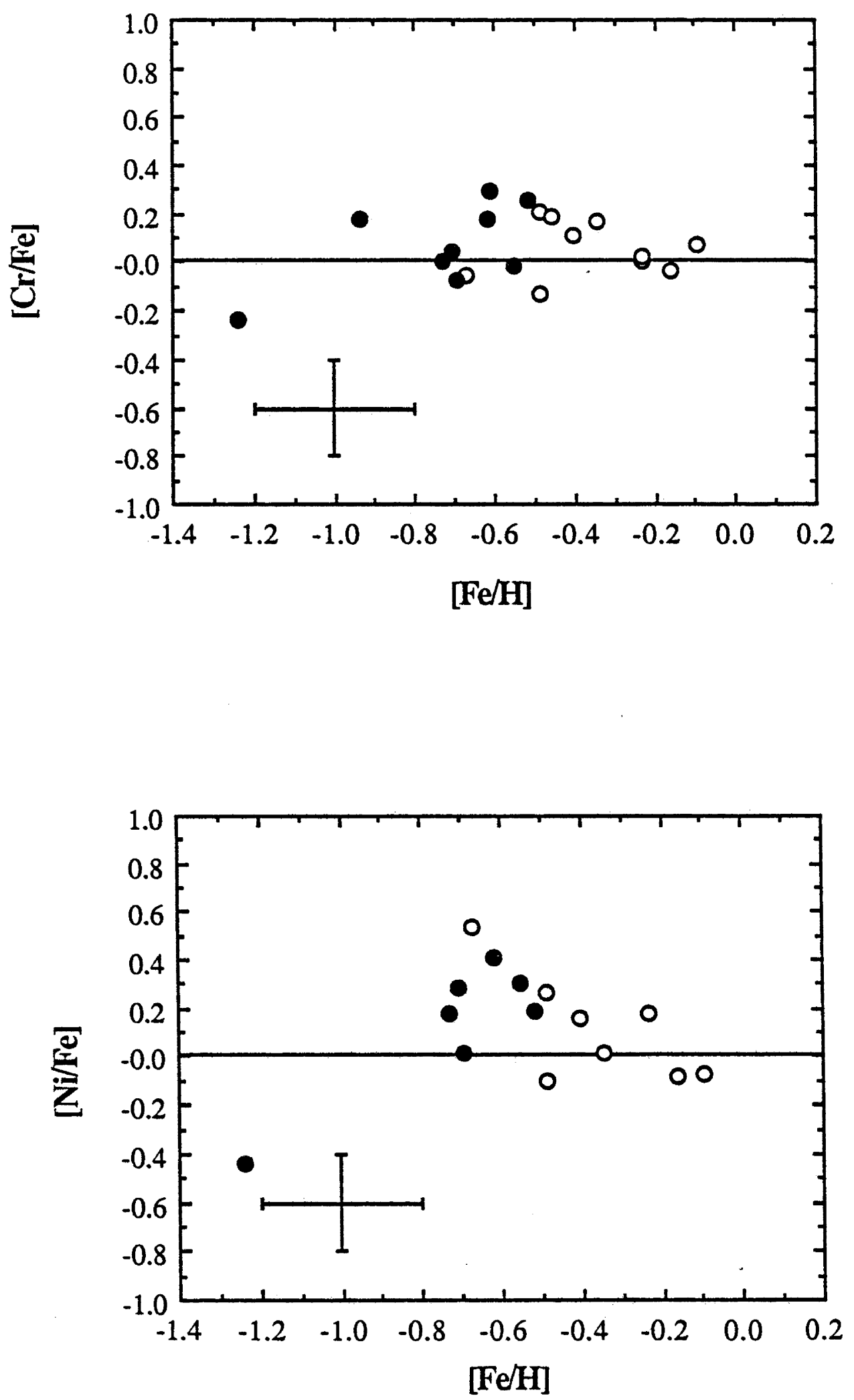
Type II, it would indeed be surprising if their abundances did not correlate as well as they do.

\section{$f$. The neutron-capture elements}

Beyond the Fe-peak elements (except for $\mathrm{Zn}$ ), the only process capable of producing elements, is that of neutron-capture (ignoring several processes of trivial efficiency required to explain the abundances of certain rare isotopes). Traditionally the neutron-capture reactions have been split into the slow $s$-process and the rapid $r$ process. The $s$-process is assumed to happen during quiescent burning phases in the lifetimes of intermediate mass stars, and hence are efficient at producing the elements that lie along the, so called, zone of $\beta$-stability. The $r$-process, however, is assumed to be effective only during times of intense neutron flux, as during a supernova explosion. Under such conditions, the neutron captures are more rapid than the $\beta$-decay times, thus producing elements that lie on the neutron rich side of the valley of $\beta$-stability (Burbidge, Burbidge, Fowler, and Hoyle 1957). Since the $\beta$-decay times are highly variable, so to are the fluxes required to overcome them, hence more gentle sites for the production of $r$-process elements are conceivable, than those of supernova explosions. This all adds to the complexity of interpreting the causes of any particular abundance pattern. In 1981, Truran proposed that, for the extremely metal weak halo stars $([\mathrm{Fe} / \mathrm{H}]<-2.0)$, only the $r$-process reactions have contributed to the abundances of the neutron-capture elements. This has received some support in recent years (Gilroy et al. 1988; Wheeler, Sneden, and Truran 1989), through comparing the observed abundances patterns for the neutroncapture elements with the theoretical abundance patterns produced solely by the $r$ - or $s$-processes. 
In the light of these recent papers, we have plotted in Figure 8 the abundance patterns for the observable elements heavier than $Z=37$, normalized at $\mathrm{Nd}$ (as is the fashion, although it is not self-evidently the best), for both Canopus (as a representative of the local disc population) and the average of the halo star abundances used by Gilroy et al. (1988) and Wheeler, Sneden, and Truran (1989). Superimposed on these we have added the $r$-and $s$-process contributions to the solar abundances derived by Cameron (1982a). This type of diagram, however, is not very clear, since it is difficult to ascertain the critical elements that are fitted better than an average disc star by one process rather than another.

In an attempt to clarify the situation, we have plotted the data from the Galactic halo and the Magellanic Clouds in Figures $9 a$ to $9 c$ so that the abundances are normalized to those of the Sun. Then, any differences from this abundance pattern become obvious by their deviations from the horizontal line. In all three figures we have normalized all the data at $Y(Z=39)$ since this seems to be in relatively constant proportion to $\mathrm{Fe}$, especially for the Magellanic Clouds (see paper I). In Figure $9 a$ we have superimposed over these data the solar system contributions by the $r$ - and $s$-processes normalized so that their sum is equal to zero (in the $\log$ ) at $\mathrm{Y}$ (Cameron $1982 a$ ). We have also computed a model whereby the full $r$-process contribution to the solar system has been added to half of the $s$ process contribution, and the result has been normalized to zero at $Y$. None of these curves fit either the Galactic halo or the Magellanic Clouds. From this we may conclude that the neutron processing history of the Clouds and the Galactic halo have been markedly different to that of the Sun. Even a fifty percent increase in the contribution by the $r$-process relative to the $s$-process is insufficient to account for the observations. However it is also apparent that any combination of $s-$ and $r$ processes that include at least half the solar $s$-process contribution would fit the Magellanic Cloud data for the light neutron-capture elements $(\mathrm{Sr}, \mathrm{Y}, \mathrm{Zr})$, whereas the Galactic halo has an entirely different distribution of these elements. 
Figure 8 A plot of the neutron-capture elements from $\operatorname{Sr}(\mathrm{Z}=38)$ to $\mathrm{Dy}(\mathrm{Z}=66)$ for the Galactic halo (closed circles) and Canopus (open circles), against the atomic number $Z$. The data and the contributions to the solar system abundances from the s-process (solid line), and the r-process (dashed line), are all normalised at Nd. 


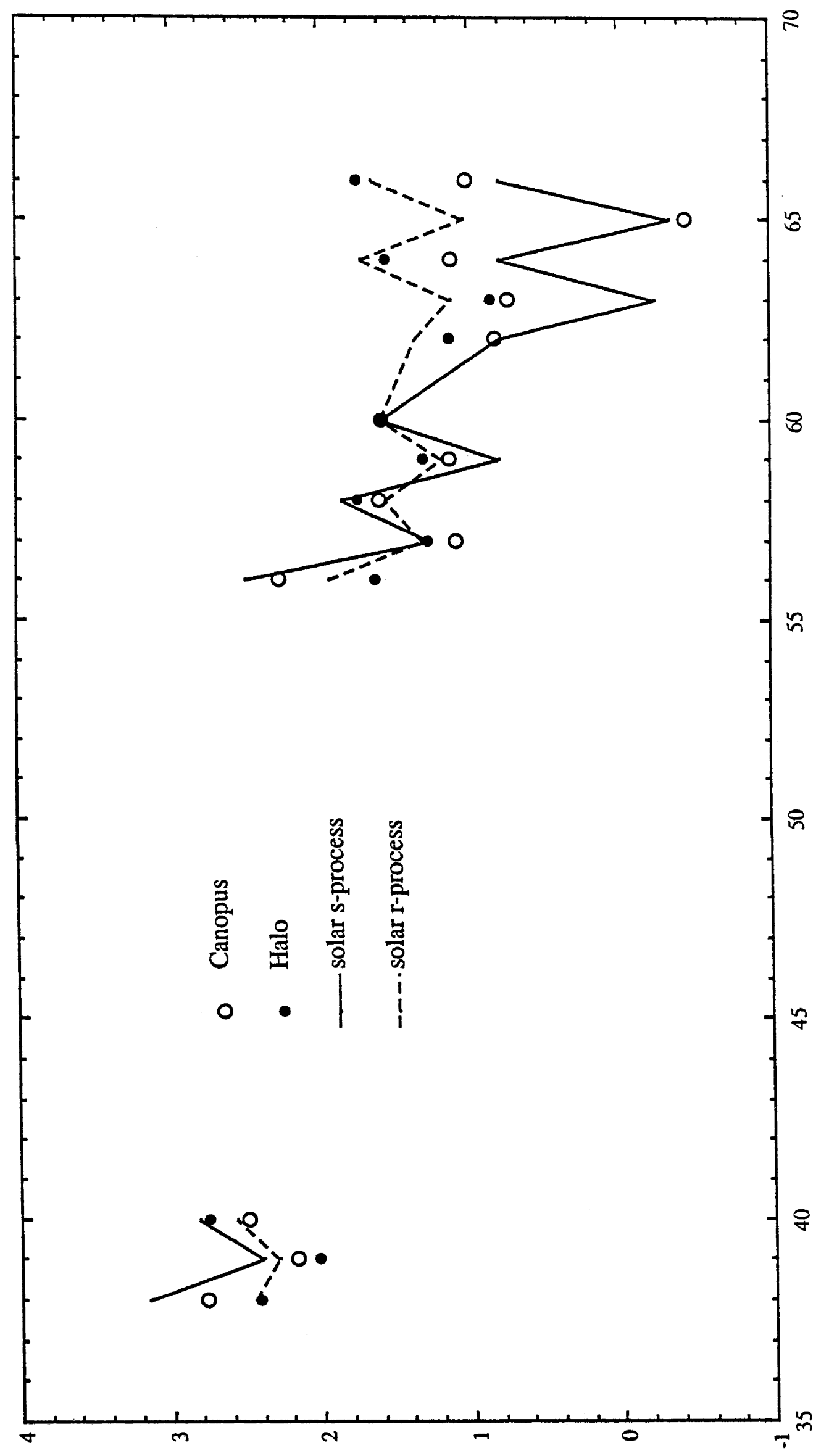

3 ㅇำ 
Figure 9a The data $[\mathrm{M} / \mathrm{H}]$ from the Galactic halo (closed circles), the SMC (open triangles), and the LMC (open circles), are normalised at $\mathrm{Y}$, and plotted against $Z$. The solid and the long dashed lines represent the contributions from the sprocess, and the r-process respectively, to the solar abundances, normalised so that their sum is equal to zero. The short dashed line represents the sum of the $r-$ process and half the s-process contributions to the solar abundances, normalised at $Y$. The error bars for the LMC, due only to the observed scatter in the abundances (see paper I), are included in this diagram for illustrative purposes.

Figure $9 \mathrm{~b}$ As for the previous figure, the data $[\mathrm{M} / \mathrm{H}]$ from the Galactic halo (closed circles), the SMC (open triangles), and the LMC (open circles), are normalised at $\mathrm{Y}$, and plotted against $\mathrm{Z}$. The solid line is the solar s-process contribution, the long dashes are due to an s-process model with an exponential neutron exposure (see the text), whilst the short dashes are from a single exposure s-process model.

Figure 9c As for the previous figure, the data $[\mathrm{M} / \mathrm{H}]$ from the Galactic halo (closed circles), the SMC (open triangles), and the LMC (open circles), are normalised at $Y$, and plotted against $Z$. The solid line is the solar r-process contribution, the long dashes are due to the r-process model of Wheeler, Sneden and Truran (1989), whilst the short dashes are due to the r-process model of Gilroy et al. (1988). See Figure 9a for illustrative error bars. 


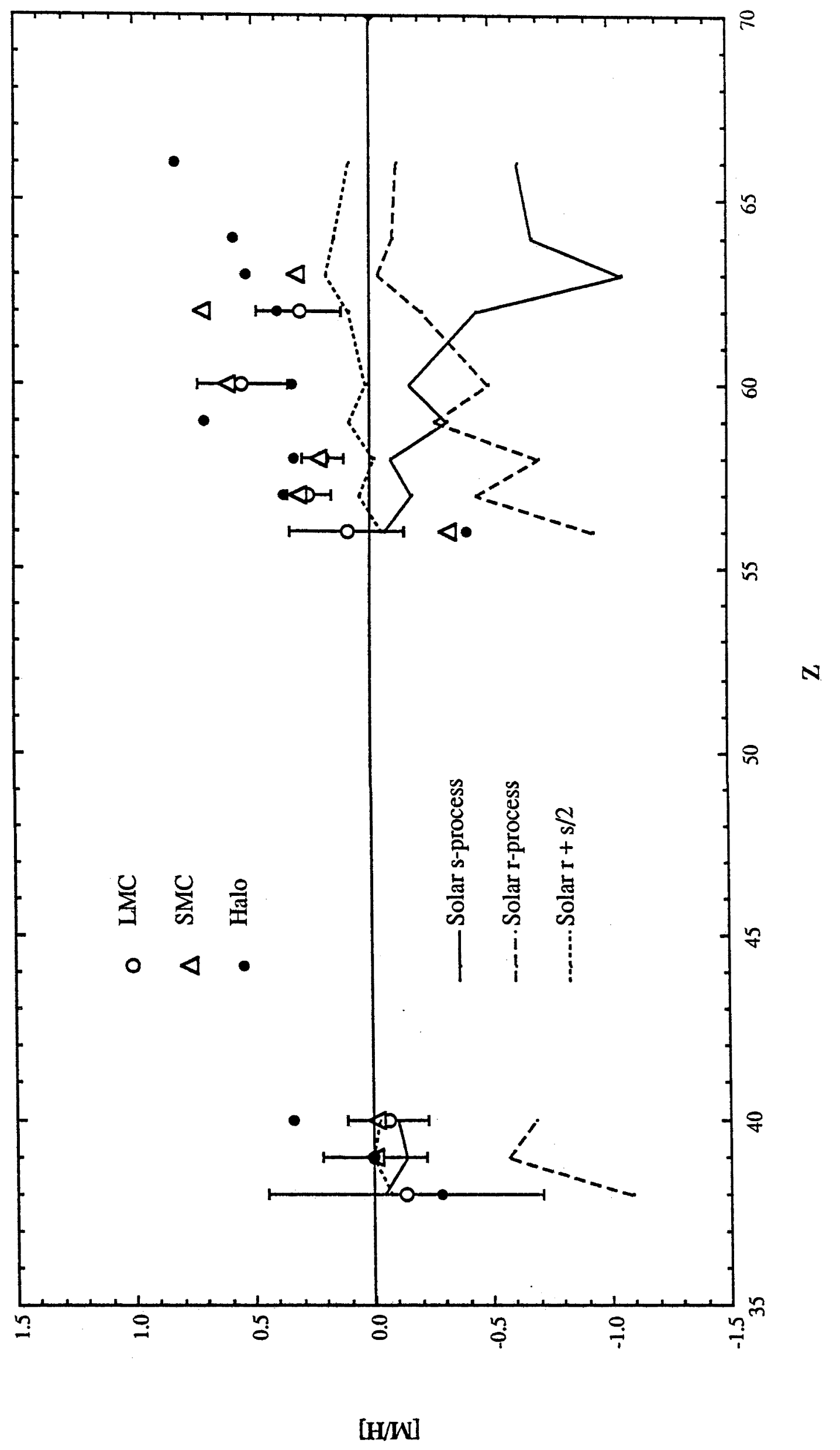




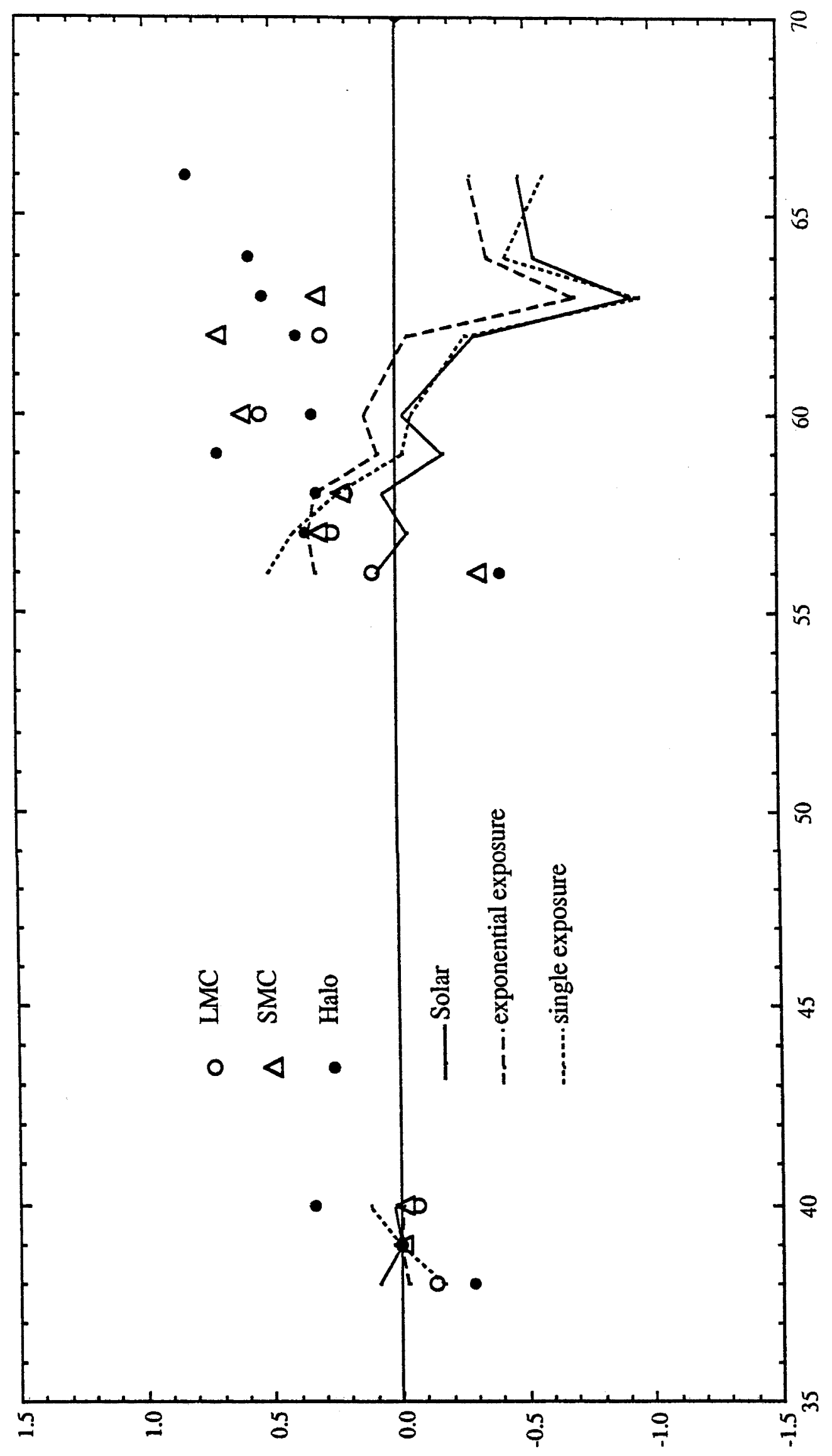

[H/W] 


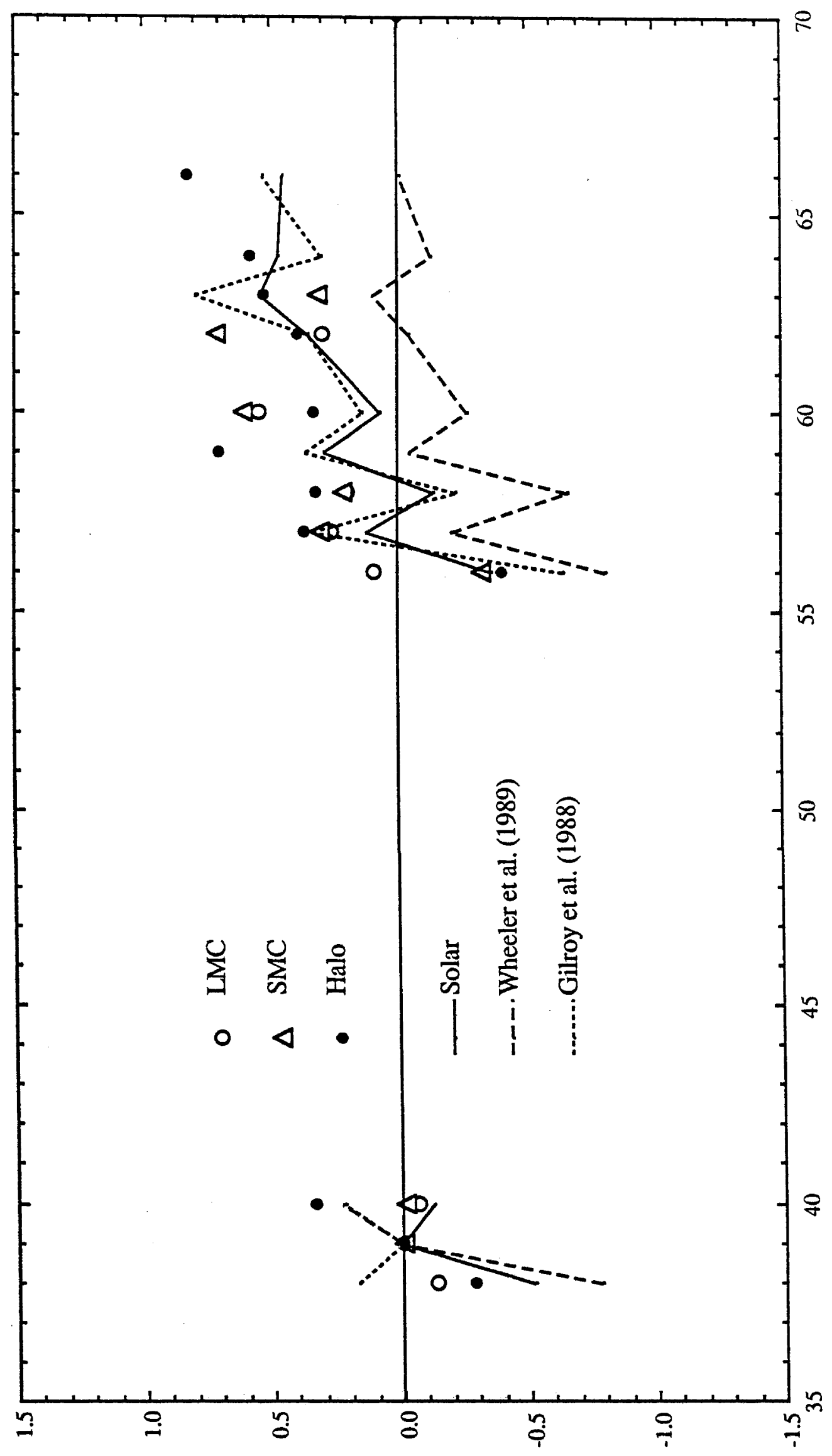

[H/W] 
In Figure $9 b$ we superimpose over the data the Solar $s$-process contributions from Cameron (1982a) normalized at Y, as well as two theoretical models of $s$ processing. One model is the single neutron exposure model of Malaney (1987a) for a flux of $1.0 \mathrm{mb}^{-1}$ and a neutron density of $10^{8} \mathrm{~cm}^{-3}$, as used by both Gilroy et al. (1988) and Wheeler, Sneden, and Truran (1989). A more correct model would be one having an exponential distribution of neutron exposures $\tau$, of the form $\exp (-$ $\tau / \tau_{0}$ ), where the constant $\tau_{0}$ is referred to as the mean neutron exposure, since this was shown by Seeger, Fowler, and Clayton (1965) to be capable of reproducing the observed solar system $s$-process abundances. An $s$-process model of this sort is, therefore, included in Figure $9 b$, having $\tau_{0}=0.5 \mathrm{mb}^{-1}$ and a neutron density of $10^{8}$ $\mathrm{cm}^{-3}$ (Malaney 1987b). The diagram shows that no significant error is introduced by assuming just a single neutron exposure, and more importantly, it confirms that no purely $s$-process model is capable of reproducing the abserved abundance distribution for $Z>55$ in the Clouds or the Galactic halo. The pure $s$-process is, however, capable of reproducing abundance distribution of the light neutron-capture elements in the Magellanic Clouds.

In Figure $9 c$ we superimpose on the data the Solar $r$-process contributions from Cameron (1982a) normalized at $\mathrm{Y}$, and two $r$-process models derived from Gilroy et al. (1988) and Wheeler, Sneden, and Truran (1989) respectively. We see from this that the Solar $r$-process contributions and the model due to Gilroy et al. (1988) both fit the observed abundance distribution beyond $Z=55$, for both the Galactic halo and the Magellanic Clouds, at least qualitatively. Indeed, there is little obvious difference between the distributions of the Clouds and the halo in this range of elements, except perhaps, a high $\mathrm{Ba}$ abundance in the LMC which suggests a contribution from $s$-processing for this element. For the light neutron-capture elements, however, none of the models is capable of reproducing any of the results, although the large difference in predicted $\mathrm{Sr}$ abundances between the models of 
Wheeler, Sneden, and Truran (1989) and Gilroy et al. (1988) suggest that this is simply a modeling problem.

We conclude that the $s$-process has been ineffective in producing elements as heavy, or heavier, than $\operatorname{Sr}(Z=38)$ in the Galactic Halo, $\mathrm{Ba}(\mathrm{Z}=56)$ in the SMC, and $\mathrm{La}(\mathrm{Z}=57)$ in the $\mathrm{LMC}$.

Why this should be the case is clearly a matter for speculation at the present time. However, it may be that, for some reason, the $s$-process neutron flux within the Cloud stars was of insufficient intensity, or duration, to be able to form the heavier elements. This might occur if, for instance, the number of thermal pulses experienced by stars climbing the asymptotic giant branch (AGB) is reduced in the Clouds relative to the Galactic disk (which in turn, may result from the lower dust content of the Clouds). If this were the case, then we would expect to find that the $[\mathrm{Ba} / \mathrm{Y}]$ ratio falls more steeply with decreasing $\mathrm{Ba}$ abundance in the Clouds than in the Galaxy. The reason for this is that $\mathrm{Ba}$ requires a higher neutron irradiation than $\mathrm{Y}$ for its formation. The results for the Clouds are plotted in Figure 10 together with a least-squares fit and the line that best fits the Galactic data (from Spite and Spite 1978). There is some evidence from this that the slope for the Clouds is indeed steeper, thus supporting our conjecture.

In Figures $11 a$ and $11 b$, we plot against $[\mathrm{Fe} / \mathrm{H}]$, the average light neutroncapture, and heavy-neutron capture abundances respectively. The light neutroncapture elements are defined here to be half the sum of the $\mathrm{Y}$ and $\mathrm{Zr}$ abundances relative to Fe for those stars where both were measured. Whilst the heavy-neutron capture abundances $\left[\mathrm{M}_{\mathrm{hn}} / \mathrm{Fe}\right]$, are defined here to be:

$$
[\mathrm{M} n / \mathrm{Fe}]=1 / 4([\mathrm{La} / \mathrm{Fe}]+[\mathrm{Ce} / \mathrm{Fe}]+[\mathrm{Nd} / \mathrm{Fe}]+[\mathrm{Sm} / \mathrm{Fe}])
$$

where at least two elements were measured from any star, and those that were otherwise missing were replaced with values maintaining the average abundance difference observed between that element and $\mathrm{Ce}$. The light neutron-capture elements show no obvious deviation from strict proportionality with $[\mathrm{Fe} / \mathrm{H}]$. This 
Figure 10 The $[\mathrm{Ba} / \mathrm{Y}]$ ratio plotted against $[\mathrm{Ba} / \mathrm{Fe}]$ for the $\mathrm{LMC}$ (open circles), and the SMC (closed circles). The dashed line is the result for the Galaxy derived by Spite and Spite (1978). A 0.2 dex error bar has been included as an indication of the errors and the scale. 


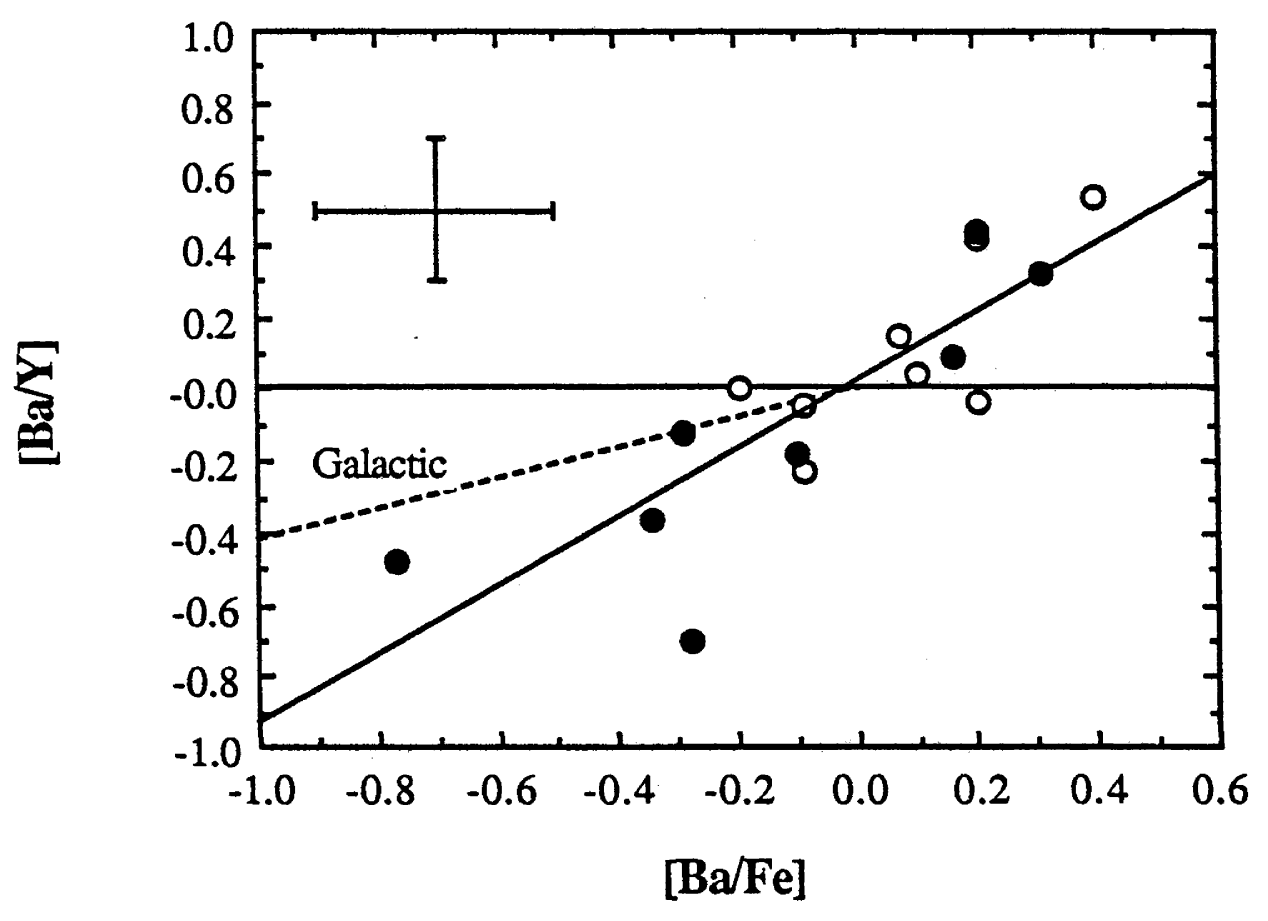


Figure 11 A plot of (a) the [light neutron-capture elements/Fe] ratio, and (b) the [heavy neutron-capture elements/Fe] ratio, versus $[\mathrm{Fe} / \mathrm{H}]$ for the LMC (closed circles), and the SMC (open circles). A 0.2 dex error bar has been included as an indication of the errors and the scale. 

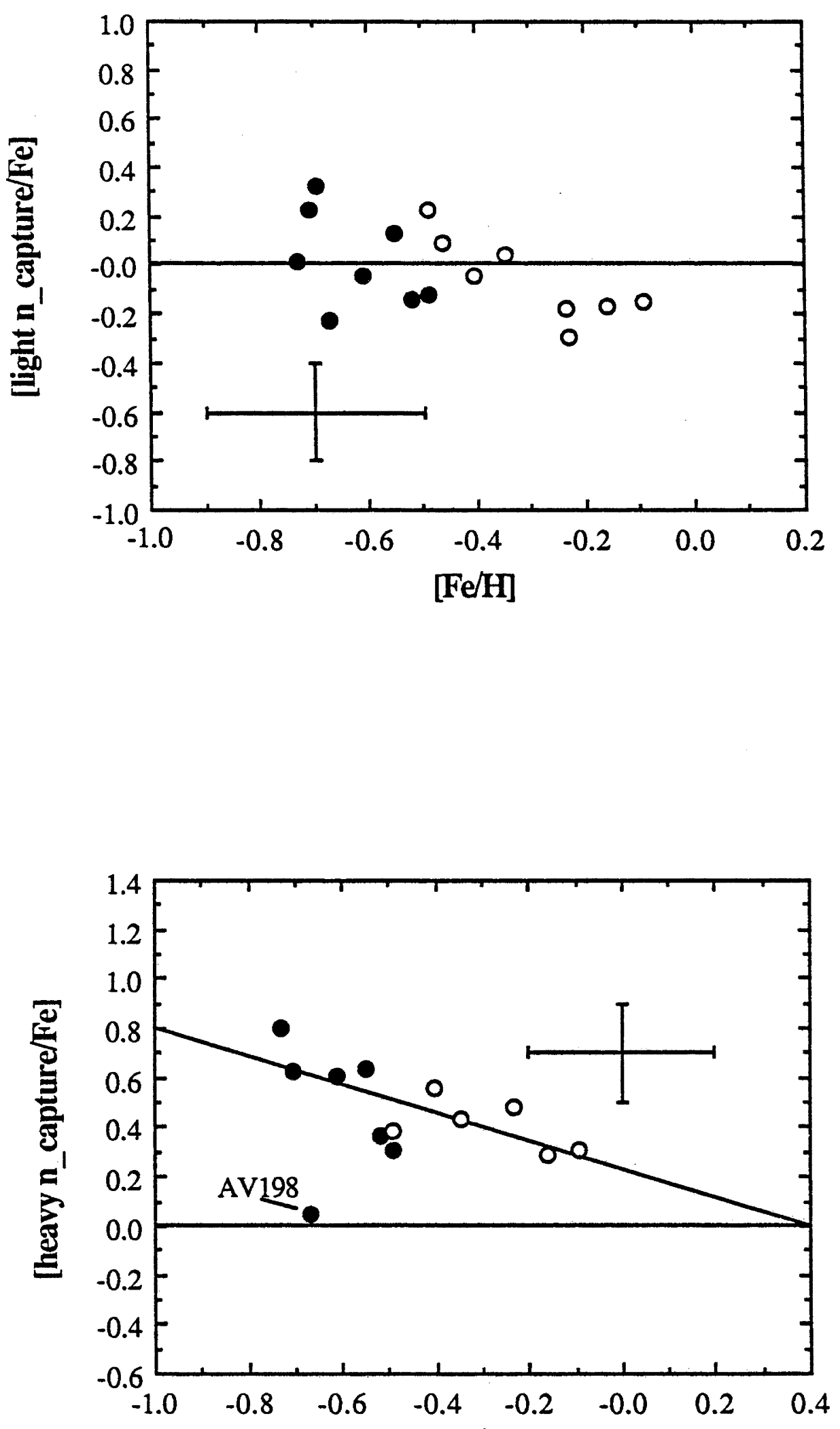

$[\mathrm{Fe} / \mathrm{H}]$ 
supports our conclusions above, that these elements show no differences from the abundances relative to $\mathrm{Fe}$ found in our Galaxy. For the heavy neutron-capture elements, however, except for the apparently discrepant point from AV 198 (see paper I), the points define a remarkably tight relationship of decreasing abundance with increasing $[\mathrm{Fe} / \mathrm{H}]$. This is in contrast to the strict proportionality with $\mathrm{Fe}$ found in the Galaxy at these metallicities (Luck and Bond 1985; Magain 1989), and suggests that the rate of production of these elements at present is less than half the iron production rate.

It is generally believed that high mass stars are the major contributors to the $r$ process elements, however, the high mass slope of the IMF in both Clouds is steeper than in the Galaxy, which indicates that there has been proportionally fewer high mass stars formed in the Clouds than in the Galaxy. It must be, therefore, that the stars themselves are producing the $r$-process elements more efficiently than in the Galaxy. Conversely, the lack of any sign of $s$-processing beyond $Z=56$, suggests that the intermediate stellar masses responsible for their production have been less efficient in the Clouds than in the Galaxy. The high barium abundance in the LMC suggests that the efficiency of the s-processing of the heavy elements may be progressively decreasing as the mass of the galaxy decreases. Several physical factors correlate with the mass of a galaxy, however, so the one that is ultimately responsible for the different production efficiencies of the heavy elements between galaxies, is open to conjecture.

\section{g. The overall abundance pattern}

In Table 2 we give the overall abundances which we have chosen as representative of the Magellanic Clouds, when due consideration has been given to all sources of data, from the literature as well as from our own work (papers I and II). Column (3) gives the abundances by number for the sun from Cameron (1982b) (except for the Ti abundance which comes from Blackwell, Shallis, and 
TABLE 2

Adopted Abundances

\begin{tabular}{|c|c|c|c|c|c|c|c|c|c|}
\hline $\begin{array}{l}\text { Elem. } \\
\text { (1) }\end{array}$ & $\begin{array}{c}Z \\
(2)\end{array}$ & $\begin{array}{c}\text { Sun } \\
(3)\end{array}$ & $\begin{array}{l}\text { Can/ } \\
\text { Sol.vic. } \\
\text { (4) }\end{array}$ & $\begin{array}{c}\text { LMC } \\
(5)\end{array}$ & $\begin{array}{l}\text { igma } \\
\text { (6) }\end{array}$ & $\begin{array}{r}\text { SMC } \\
(7)\end{array}$ & $\begin{array}{c}\text { Sigma } \\
(8)\end{array}$ & $\begin{array}{l}\text { LMC - } \\
\text { Sol.vic. } \\
\text { (9) }\end{array}$ & $\begin{array}{l}\text { MC - } \\
\text { Sol.vic. } \\
(10)\end{array}$ \\
\hline 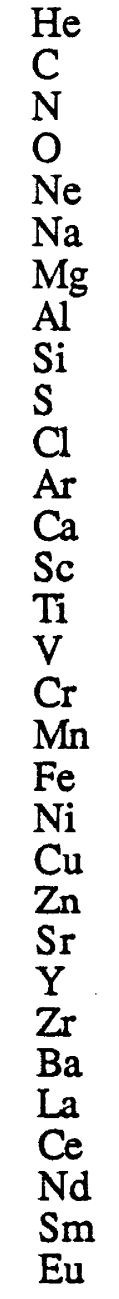 & $\begin{array}{r}2 \\
6 \\
7 \\
8 \\
10 \\
11 \\
12 \\
13 \\
14 \\
16 \\
17 \\
18 \\
20 \\
21 \\
22 \\
23 \\
24 \\
25 \\
26 \\
28 \\
29 \\
30 \\
38 \\
39 \\
40 \\
56 \\
57 \\
58 \\
60 \\
62 \\
63\end{array}$ & $\begin{array}{l}10.83 \\
8.62 \\
7.94 \\
8.84 \\
7.99 \\
6.35 \\
7.60 \\
6.50 \\
7.58 \\
7.27 \\
5.25 \\
6.60 \\
6.37 \\
3.07 \\
5.08 \\
3.98 \\
5.68 \\
5.54 \\
7.53 \\
6.25 \\
4.31 \\
4.68 \\
2.93 \\
2.26 \\
2.65 \\
2.26 \\
1.14 \\
1.65 \\
1.47 \\
0.96 \\
0.55\end{array}$ & $\begin{array}{r}-0.29 \\
-0.37 \\
-0.14 \\
-0.09 \\
0.23 \\
-0.03 \\
-0.57 \\
0.05 \\
-0.21 \\
-0.09 \\
-0.18 \\
-0.25 \\
-0.22 \\
-0.10 \\
-0.10 \\
-0.11 \\
-0.14 \\
-0.11 \\
0.00 \\
-0.18 \\
-0.01 \\
-0.15 \\
-0.08 \\
-0.15 \\
0.00 \\
-0.05 \\
-0.06 \\
0.11 \\
-0.14 \\
0.18\end{array}$ & $\begin{array}{l}7.72: \\
6.81 \\
4.77 \\
6.29 \\
5.97 \\
2.64 \\
4.85 \\
4.05 \\
5.41 \\
5.19 \\
7.23 \\
5.96\end{array}$ & $\begin{array}{c}0.13 \\
\ldots \\
\ldots \\
0.09 \\
0.08 \\
0.25 \\
0.16 \\
0.21 \\
0.09 \\
0.22 \\
0.14 \\
0.32 \\
0.18 \\
0.09\end{array}$ & $\begin{array}{l}10.91 \\
7.76 \\
6.63 \\
8.03 \\
7.27 \\
6.08 \\
6.99 \\
6.40: \\
7.31 \\
6.59 \\
4.7 \\
5.81 \\
5.84 \\
2.38 \\
4.49 \\
3.78 \\
5.13 \\
5.03 \\
6.89 \\
5.86 \\
3.73:\end{array}$ & $\begin{array}{c}0.18 \\
0.15 \\
0.20 \\
0.08 \\
0.20 \\
0.15 \\
0.15 \\
0.27 \\
0.20 \\
0.31 \\
0.13 \\
0.20 \\
\ldots \\
\ldots \\
\ldots . . \\
0.23 \\
0.22 \\
0.50 \\
0.30 \\
0.27 \\
0.12 \\
0.39 \\
0.08\end{array}$ & $\begin{array}{r}0.19 \\
-0.36 \\
-0.40 \\
-0.13 \\
-0.15 \\
-0.21 \\
-0.24 \\
0.17 \\
-0.06 \\
-0.21 \\
-0.19 \\
-0.30 \\
.0 .39 \\
-0.39 \\
-0.31 \\
-0.24 \\
-0.23 \\
-0.02 \\
-0.07 \\
0.10 \\
0.11 \\
. . .\end{array}$ & $\begin{array}{l}-0.09 \\
-0.60 \\
-0.94 \\
-0.67 \\
-0.63 \\
-0.62 \\
-0.59 \\
-0.30: \\
-0.60 \\
-0.47 \\
-0.46 \\
-0.61 \\
-0.43 \\
-0.52 \\
-0.49 \\
-0.34 \\
-0.47 \\
-0.37 \\
-0.58 \\
-0.40 \\
-0.40:\end{array}$ \\
\hline
\end{tabular}


Simmons 1982), on a scale of $12+\log N(M / H)$. In column (4) we give the abundances of the local interstellar medium (ISM) relative to the Sun (in the sense ISM-Sun), where the elements heavier than $\mathrm{Z}=18$, and the lighter elements $\mathrm{C}, \mathrm{Na}$, $\mathrm{Mg}, \mathrm{Al}$, and Si are all derived from Canopus (Lyubimkov and Boyarchuk (1982) if the element was observed, otherwise from Desikachary and Hearnshaw (1982) or the more recent paper by Reynolds, Hearnshaw, and Cottrell 1988), while the remaining elements come from the work on Galactic H II regions by Shaver et al. (1983). Canopus was chosen to represent the stellar population of the present day ISM since it is a young, well studied supergiant, of the same type as the Magellanic Cloud stars studied in paper I (see paper I for a more detailed discussion). Columns (5) and (7) give the absolute abundances of the LMC and the SMC respectively, on the same scale as for the Sun, while the errors deduced from the scatter in the abundances are included in the adjacent columns for each Cloud. Finally, the last two columns give the abundances of the LMC and SMC respectively, relative to the local ISM as given in column (4) (in the sense Cloud-ISM). These latter abundances should be less affected by systematic errors in the analyses than the absolute abundances given in columns (5) and (7), since we compare like objects. The heavy element abundances in columns (5), (7), (9) and (10), for $Z>18$, and the elements $\mathrm{C}, \mathrm{Na}, \mathrm{Mg}, \mathrm{Al}$ and $\mathrm{Si}$, are derived from the F-supergiants studied in paper I, supplemented by the abundances of the three SMC supergiants analyzed by Spite, Spite, and François (1989), and Spite, Barbuy, and Spite (1989). The other elements are derived from our work on H II-regions and supernova remnants (paper II), with due consideration given to the abundances derived in the review by Dufour (1984) from all previous abundance estimates given in the literature.

In Figures $12 a$ and $12 b$, we plot against atomic number the LMC abundances relative to the Sun and to the local Galactic ISM respectively (as given in Table 2). 
Figure 12 The adopted distribution of abundances in the LMC (a) relative to the Sun, and (b) relative to the local ISM (we define $[M / H]^{*}=N(M / H)_{\text {object - }}$ $\left.\mathrm{N}(\mathrm{M} / \mathrm{H})_{\mathrm{ISM}}\right)$. The dashed line represents the approximate metallicity of the Cloud. The filled triangle is the $\mathrm{C}$ abundance derived from Dufour, Shields, and Talbot (1982), and Dufour, Schiffer, and Shields (1984), and the open circles are elements with insufficient data for an error bar to be estimated. 


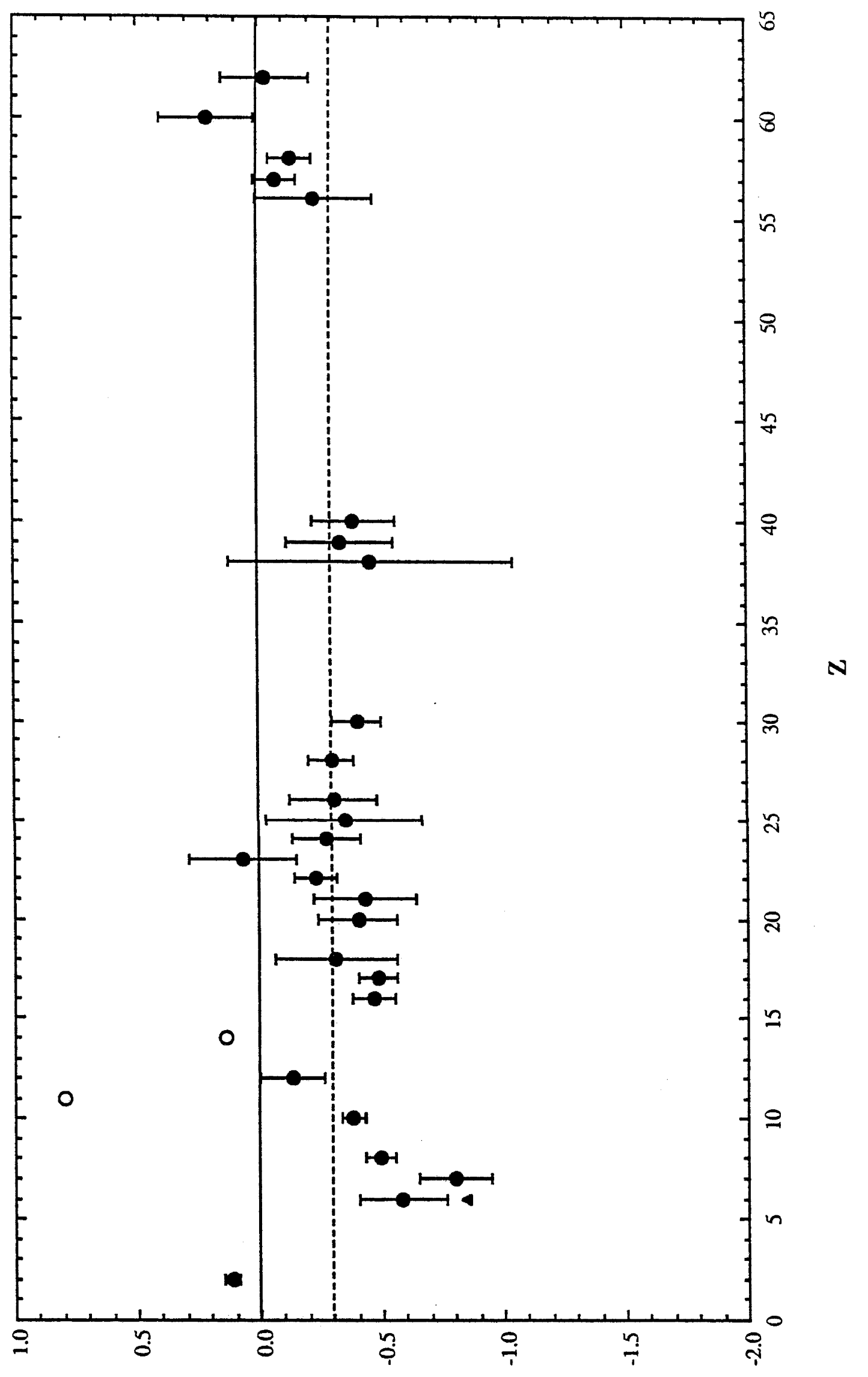

[H/W] 


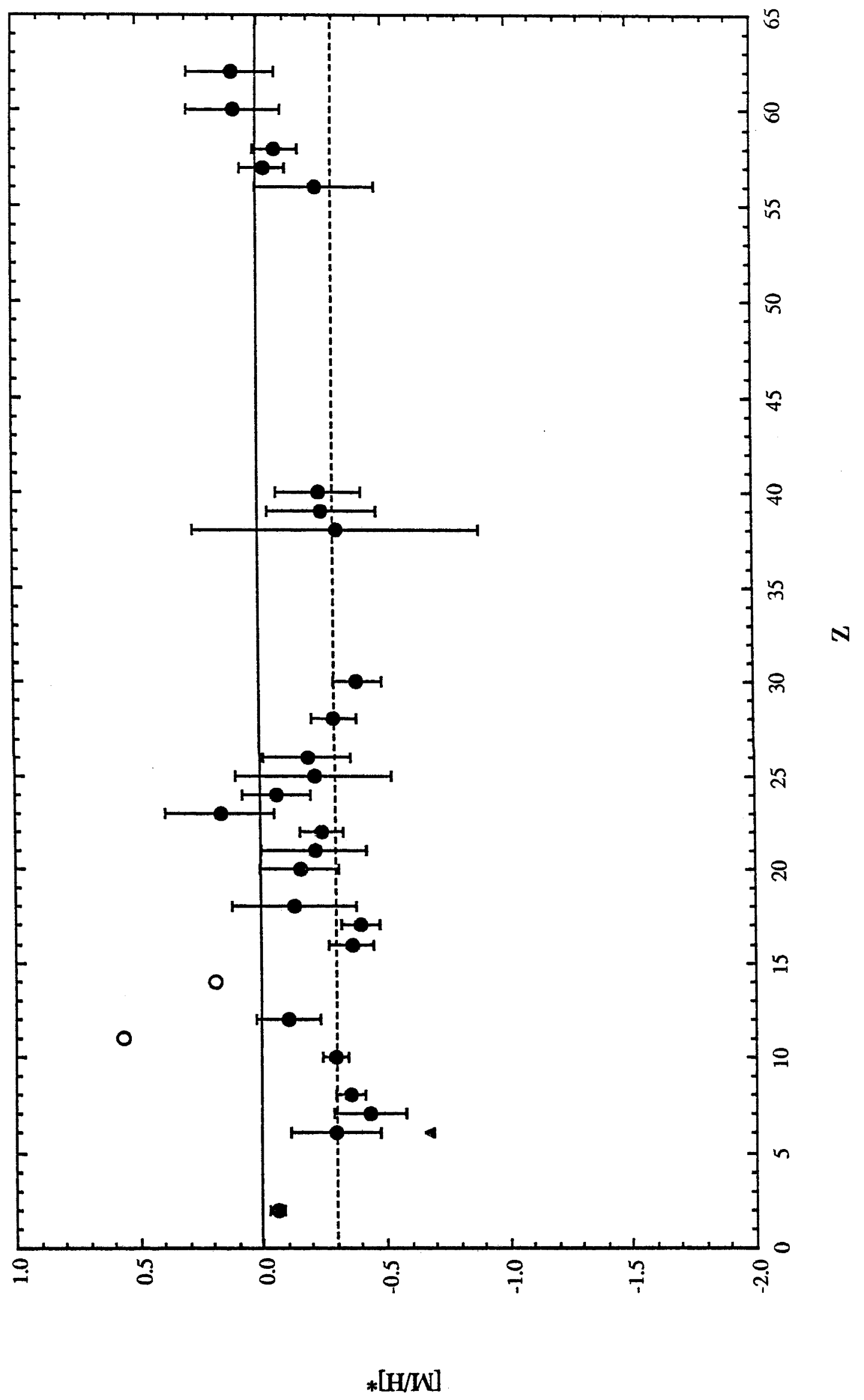


Similarly in Figures $13 a$ and $13 b$ we plot the results for the SMC. In each case, the filled triangle represents the value for carbon derived from the UV observations of $\mathrm{H}$ II regions by Dufour, Shields, and Talbot (1982), and Dufour, Schiffer, and Shields (1984), the open circles represent elements with insufficient information for us to assign an error bar, and the dashed line represents the approximate $\mathrm{Fe}$ abundance.

As noted in paper I, and shown even more clearly here, there appears to be a pronounced falling off in abundances relative to the Sun for the very lightest elements (Figures $12 a$ and 13a). Disregarding the $\mathrm{N}$ abundance which, considering the uncertainties in our present understanding of its formation, is possibly misleadingly low, we are left with a definite dropping off in relative abundances of three well observed elements; $\mathrm{C}, \mathrm{O}$, and $\mathrm{Ne}$. It must be remembered that $\mathrm{He}$ is not included in this trend due to its largely cosmic origin. This trend, however, disappears when the abundances are plotted relative to the local ISM (Figures $12 b$ and $13 b$ ). All three of the critical elements in both Clouds show an entirely normal relative abundance. This represents significant evidence that the star formation histories of the Magellanic Clouds, are more similar to the star formation history of the present day ISM in the vicinity of the Sun, than to that of the Sun itself. 
Figure 13 The adopted distribution of abundances in the SMC (a) relative to the Sun, and (b) relative to the local ISM. As for Figure 12, the dashed line represents the approximate metallicity of the Cloud; the filled triangle is the C abundance derived from Dufour, Shields, and Talbot (1982), and Dufour, Schiffer, and Shields (1984); and the open circles are elements with insufficient data for an error bar to be estimated. 


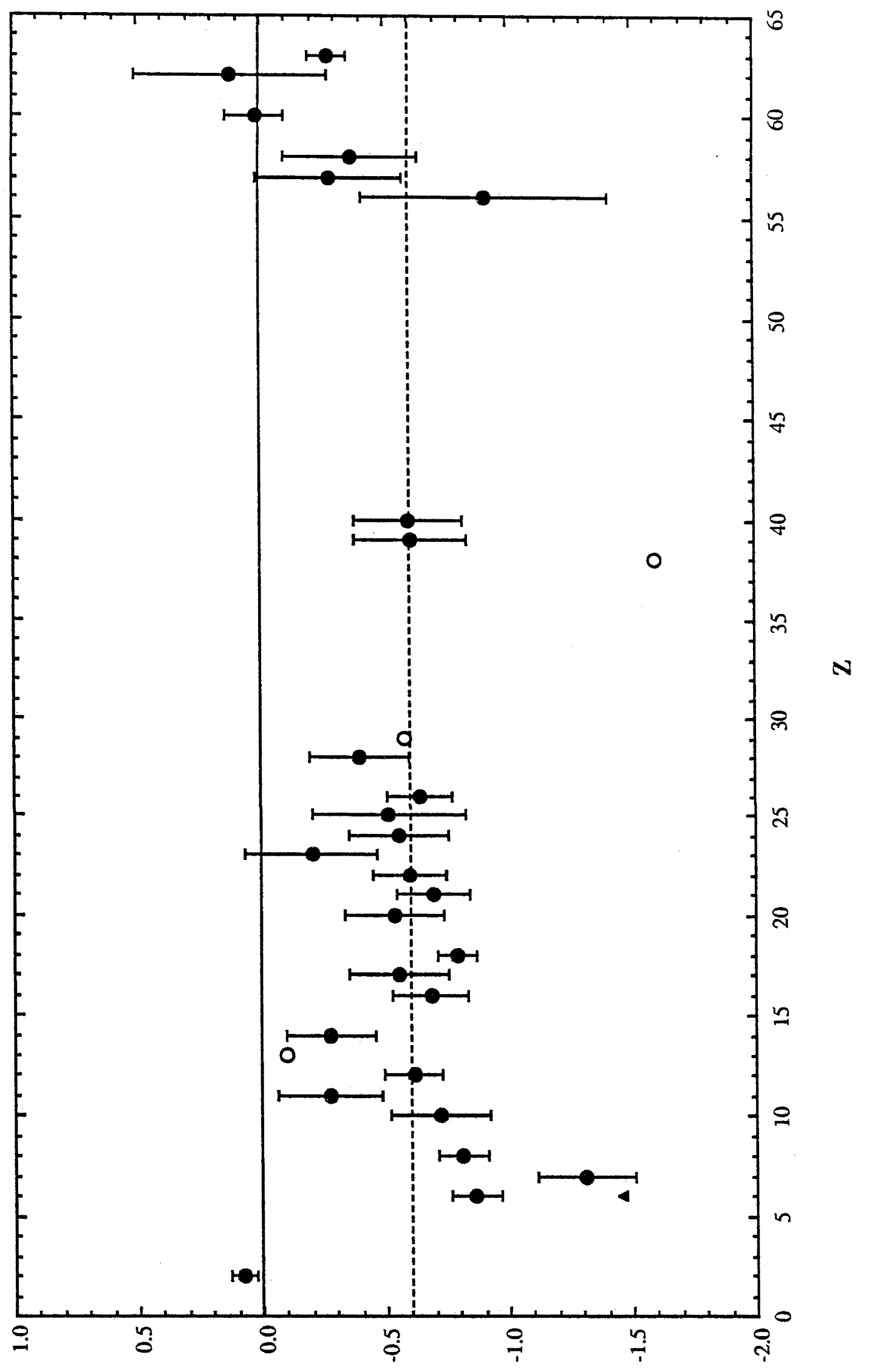

[H/W] 


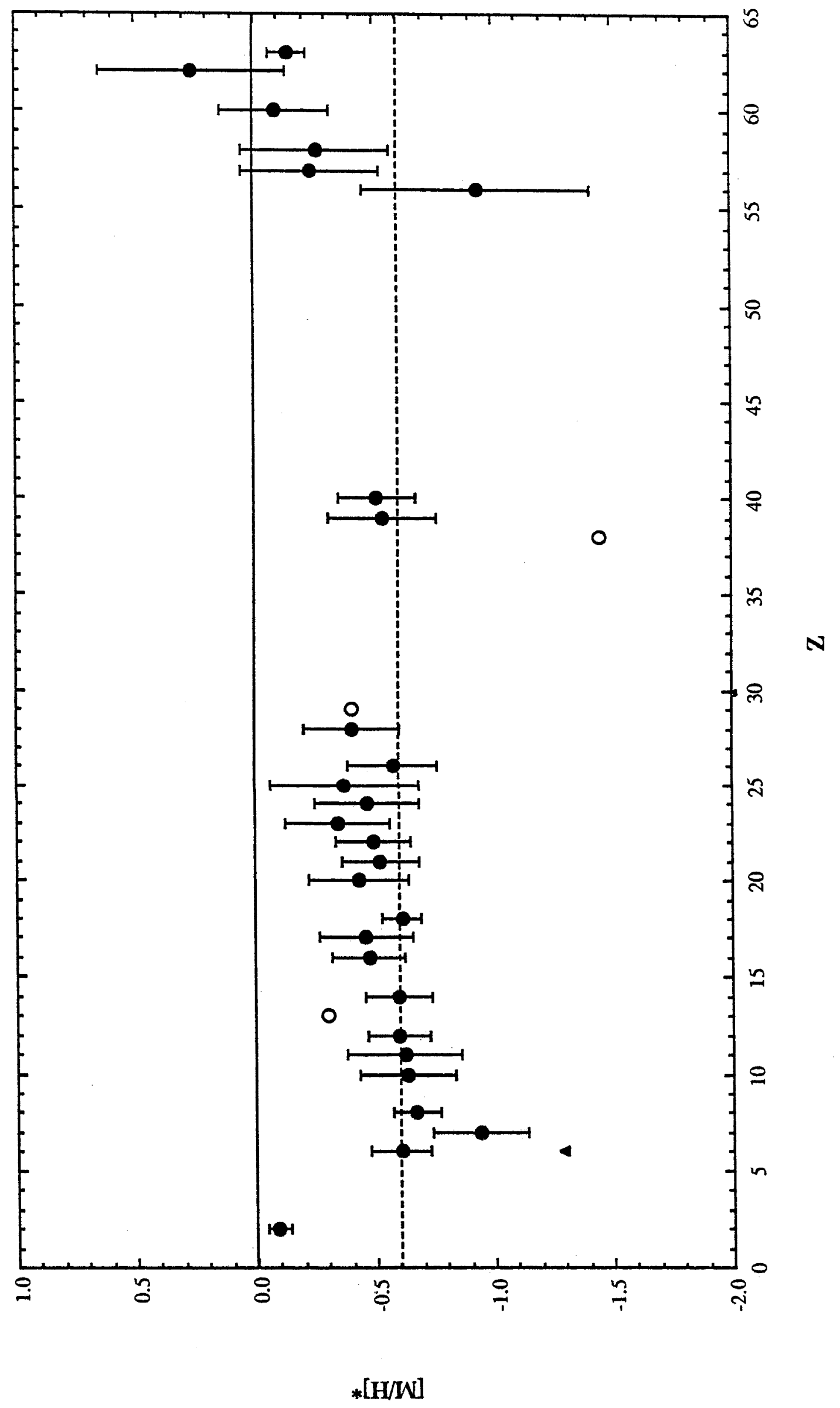




\section{DISCUSSION}

\section{a. Star formation}

In Figure $14 a$ we show the gas surface mass and the star surface mass, relative to the total systemic surface mass, in our models for both the SMC and the LMC as a function of time. This may be compared to the Galactic model derived by Dopita (1989), which is given here in Figure $14 b$. We can immediately see that the peak in gas mass, and hence the star formation rate, monotonically broadens as the mass of the galaxy decreases, as well as being progressively further delayed in time. This fits in well with the observations for all three galaxies. Observations from our own Galaxy can best be modelled assuming prompt initial enrichment (see for instance Pagel 1988). This theory requires a narrow intense peak in star formation very early in the history of the Galaxy and is well modelled by the gas mass shown in Figure $14 b$. The LMC is observed to have a broader star formation peak at a look-back-time of around 5 Gyrs (see Butcher 1977, Stryker 1981, Stryker and Butcher 1981, Frogel and Blanco 1983), and for the SMC there is some question whether any peak can be observed at all (see Bica, Dottori and Pastoriza 1986, and Frantsman 1988), but if there is one it is probably at a look-back-time of around 3 Gyrs (see Hawkins and Brück 1982, 1984). The peaks in the star formation rate were specifically fitted in our models, but they successfully predict the increasing definition of the peak with increasing galactic mass.

The specific star forming efficiencies, defined here as being the mass of stars formed at a given time divided by the total systemic mass, do not show a monotonic trend. The LMC and the Galaxy appear to have very similar specific star forming efficiencies at 8 Gyrs. The SMC on the other hand is significantly less efficient at producing stars, and a greater specific surface density of gas is allowed to build up in this galaxy than in the more massive LMC. This suggests the possible scenario whereby any galaxy is equally efficient at forming stars, as long as it is above some 
Figure 14 The gas and star surface mass densities relative to the total systemic mass for (a) the SMC and the LMC (as marked), and (b) the Galaxy. 

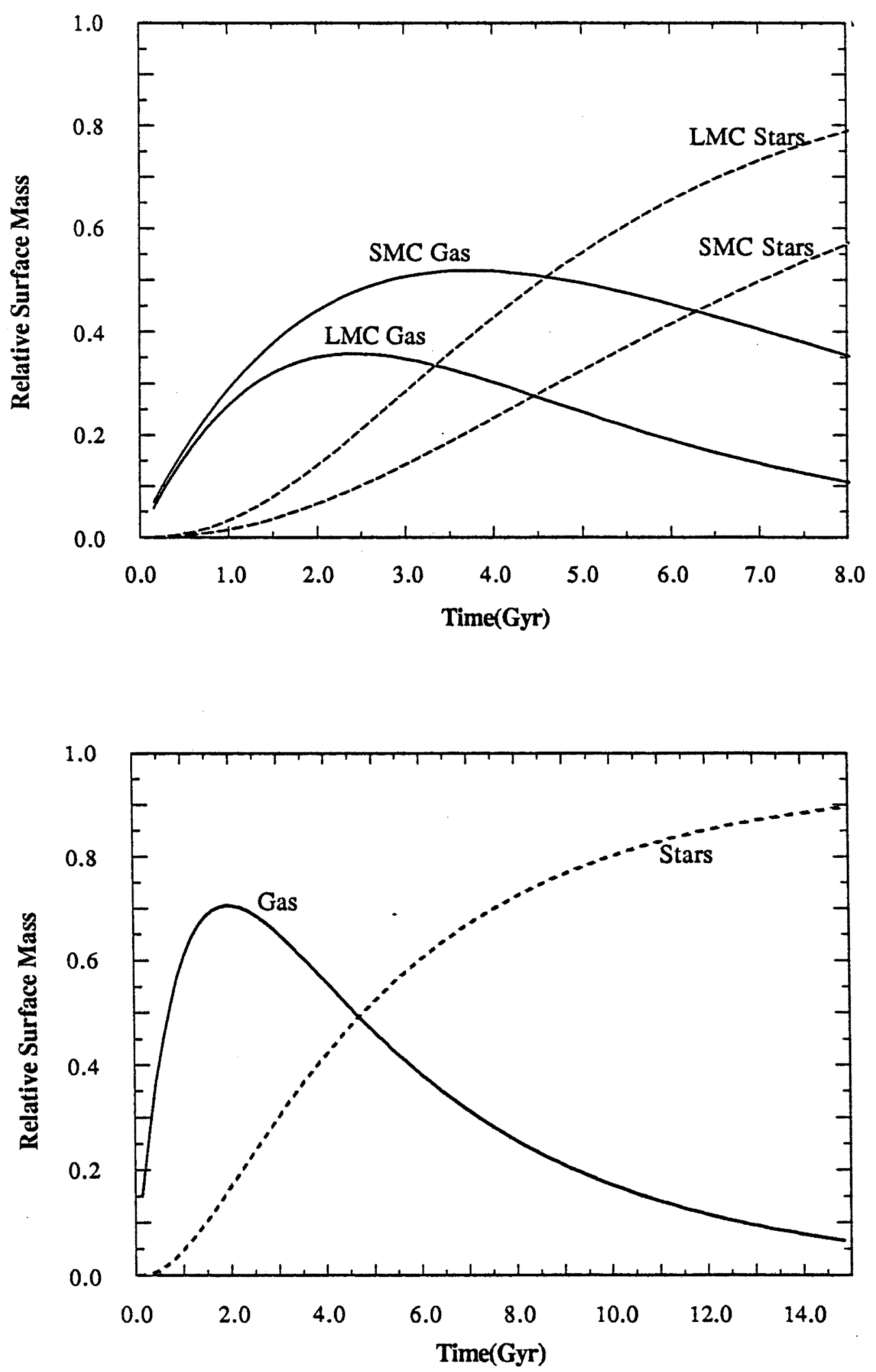
critical total mass. For less massive galaxies the star formation efficiency progressively decreases, while the gas content increases. This conjecture was tested by plotting in Figure 15, the ratio of the star mass to the total mass versus the total mass for the sample of irregular galaxies studied by Hunter, Gallagher, and Rautenkranz (1982), as well as for the Galaxy and the Magellanic Clouds. A least squares second order fit has been drawn through the data, and although the scatter is substantial (due, at least in part, to the large observational error inherent in the estimation of mass), the data is not inconsistent with our conjecture.

\section{b. The IMF}

We have shown that a steep IMF is necessary in the Magellanic Clouds to account for the observed relationship between $[\alpha$-element $/ \mathrm{Fe}]$ and $[\mathrm{Fe} / \mathrm{H}]$. This agrees with the observational results on the slope of the upper IMF in the Clouds by Humphreys and McElroy (1984). One possible explanation for such a steep IMF compared with that of the Galaxy, is to appeal to the differences in the global structures of the Clouds and the Galaxy. Shearing motions in the disks of both the LMC and the SMC must be low since their rotation velocities are low and they both have large areas of solid-body rotation. It follows that their molecular clouds will experience fewer high velocity collisions and will be more prone to low velocity coalescence. Under such conditions, we speculate that fragmentation will take place under cooler conditions than in the more violent conditions experienced in the Galaxy and other spiral galaxies, resulting in fewer high mass stars and, therefore, a steeper IMF on average.

\section{c. Radial Inflow in the Galaxy}

In $\S$ III we presented evidence that the star formation histories of the Magellanic Clouds are different to that of the Sun, purely on the grounds of the drop off in the abundances of the light elements with decreasing atomic number. 
Figure 15 The stellar mass fraction of the total systemic mass plotted against the total systemic mass, for the Galaxy and the Magellanic Clouds (open circles as marked), and the sample of irregular galaxies from Hunter et al. (1982, filled circles). The line represents a least squares second order fit to the data. 


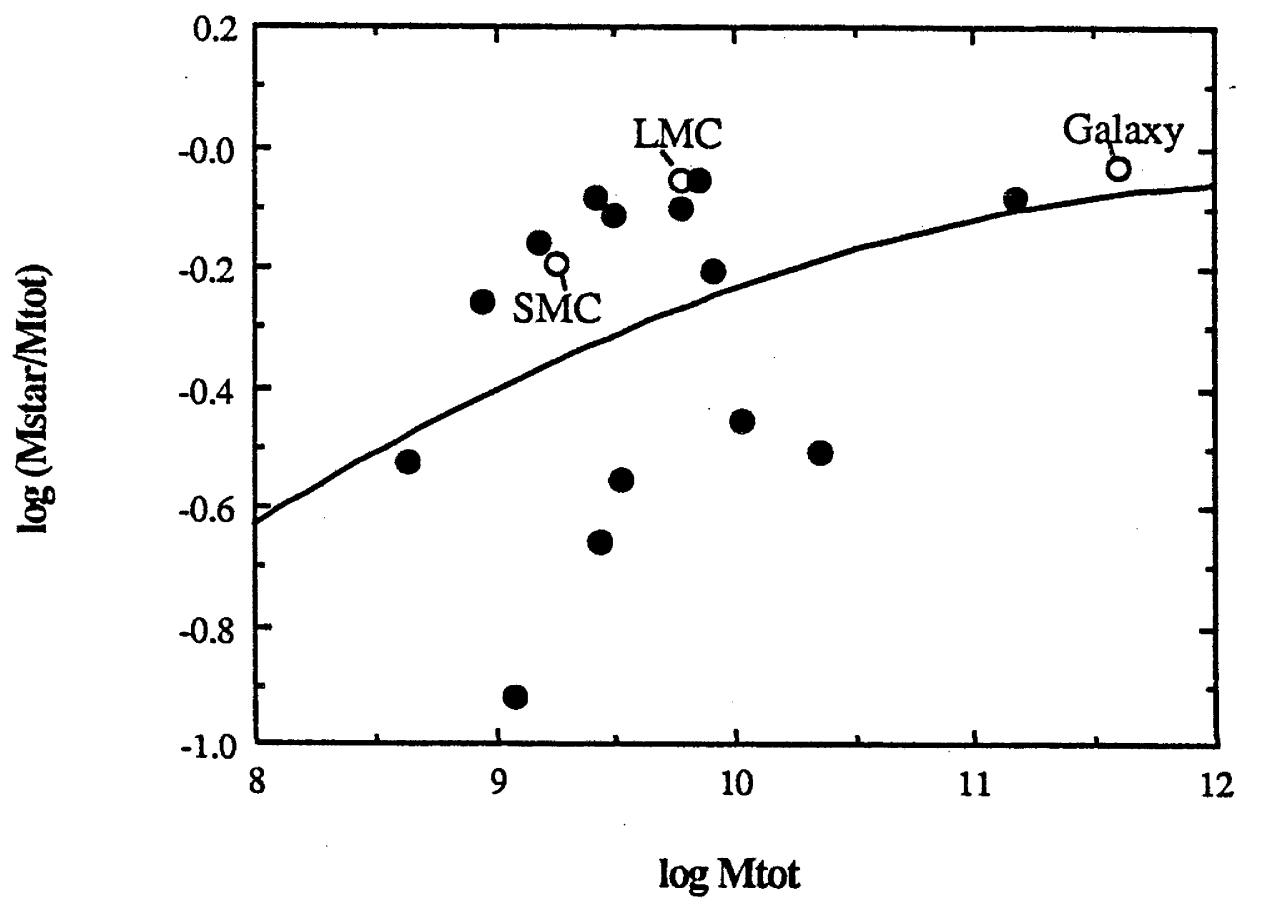


However, when comparing our results with the local Galactic ISM, rather than with the Sun, we found the disparity in the light elemental abundances disappeared. The implication is, that the Sun was born in a cloud in which more light elements were produced than is the case for the present day local ISM. Presumably, the cloud that bore the Sun has fallen more towards the center of the Galaxy, while clouds from further out, where the star formation has not been so intense, have fallen in to the present vicinity of the Sun. This represents new evidence for the reality of radial inflow.

The mechanisms that could be responsible for driving the radial inflow of gas in our Galaxy has been discussed in some detail by Dopita (1989). He comes to the conclusion that stellar orbital diffusion, through gravitational scattering by giant molecular clouds, is the most likely driving force. He shows that the stellar disk is being heated at a constant rate, and since the cloud-star interaction is energy conserving, the gaseous component must be losing energy. Since the Galaxy has a flat rotation curve, the inflow rate is proportional to the relative surface densities of the stars and the gas. This process is essentially one of dynamical friction, whereby asymmetric drift of the old stars puts a torque on the gas layers which causes them to shed orbital energy and spiral towards the center.

The other major feature of the plots of the overall abundances, is the pronounced increase in the very heavy metals $(Z>56)$ with increasing atomic number. This is, however, just a restatement of the distribution found for these elements in Figure 9 and discussed in the last section. 


\section{SUMMARY AND FUTURE WORK}

In this work we have established five major results:

1. The interstellar medium of the LMC has a mean metallicity 0.2 dex lower than the local ISM, and the metallicity of the SMC is 0.6 dex lower. However, the interstellar media of both the Magellanic Clouds and the Galaxy have significantly non-solar elemental ratios.

2. The stellar carbon abundances in the Clouds appear to be entirely normal, it is therefore the $\mathrm{H} \mathrm{II}$ regions that must be overdeficient in this element.

3. The $s$-process appears to have been as effective in the Magellanic Clouds as for the local ISM for elements as massive as $\mathrm{Zr}(Z=40)$ for the SMC, and as massive as $\mathrm{Ba}(\mathrm{Z}=56)$ for the $\mathrm{LMC}$, but to have much reduced effectiveness above these elements.

4. The $r$-process alone is successful in predicting qualitatively the increase in heavy neutron-capture elements relative to the Sun for the elements heavier than $\mathrm{Ba}$ in the LMC, and heavier than $\mathrm{Zr}$ in the SMC. The fit of the $r$-process models is as good as for the Galactic Halo.

5. The abundances of the local Galactic ISM are systematically less than those of the Sun, despite the fact that the Sun is already $\sim 4$ Gyr old. The most likely explanation for this is that there has been a radial inflow of the ISM with respect to the Sun through stellar orbital diffusion.

There is, of course, much room for improvement in this first analysis of the detailed global abundances in the Magellanic Clouds. Many critical elements need to be studied more carefully ( $\mathrm{Na}, \mathrm{Si}, \mathrm{S}, \mathrm{Sr}, \mathrm{Ba}$ and $\mathrm{Eu}$ ), while others have not been observed at all ( $\mathrm{Al}, \mathrm{Gd}$ and $\mathrm{Dy})$. The sample of objects is sometimes embarrassingly small (for example, only two SNRs were observed in the SMC). The possibility of observing some of the lighter elements by studying near main sequence B-stars, 
opens up a whole new field of study. This work is all within the reach of presentday ground-based observations, however, with the launch of the Hubble Space Telescope, new possibilities will present themselves. We would be capable of taking spectra of fainter, and therefore, older stars in the Magellanic Clouds, thus providing a longer baseline for investigating the star formation history of the Clouds.

This study has opened up a range of questions that can only be answered properly through more detailed theoretical modeling in conjunction with further observations. What causes the variations in production efficiencies of the $s$-process and $r$-process elements? Why is nitrogen anomalously underabundant in the $\mathrm{H} \mathrm{II}$ regions, and yet almost of normal abundance relative to iron in the SNRs? Why are the carbon abundances in the $\mathrm{H}$ II regions overdeficient? Why is vanadium apparently overproduced in the Clouds?

Finally, it should be noted here that the nuclear enrichment model described in this paper requires a thorough investigation of its parameter space to define more exactly the constraints it can place on the star formation history of the Clouds. This, and many of the questions described above, will be the subject of future papers.

Thanks to all those who have helped in making the observations, and to the Directors and staff of both Mount Stromlo and Siding Spring Observatories and the Anglo Australian Observatory, for the use of their facilities. The Australian Time Allocation Committee has been generous in their support for this project, and are gratefully acknowledged. Thanks also to Mike Bessell, Graeme Smith and Peter Wood for their helpful comments and suggestions. Finally, one of us (S.C.R.) would like to acknowledge the financial support received from a Commonwealth Postgraduate Scholarship during the course of this work. 


\section{REFERENCES}

Alloin, D., Collin-Souffrin, S., Joly, M., and Vigroux, L. 1979, Astr. Ap., 78, 200.

Andersen, J., Edvardsson, B., Gustafsson, B., and Nissen, P. E. 1988, in IAU Symp. No. 132 "The Impact of Very High S/N Spectroscopy on Stellar Physics", ed G. Cayrel de Strobel, M. Spite (Dordrecht: Kluwer), p. 441. Audouze, J., Chiosi, C., and Woosley, S. E. 1986, "Nucleosynthesis and Chemical Evolution" (Sauverny-Versoix: Geneva Observatory).

Augarde, R., and Lequeux, J. 1985, Astr. Ap., 147, 273.

Arnett, D. W., 1978, Ap. J., 219, 1008.

Beynon, T. G. R. 1978a, Astr. Ap., 64, 145. . 1978b, Astr. Ap., 64, 299.

Bica, E., Dottori, H., and Pastoriza, M. 1986, Astr. Ap., 156, 261.

Binette, L., Dopita, M. A., and Tuohy, I. R. 1985, Ap. J., 297, 476.

Blackwell, D. E., Shallis, M. J., and Simmons, G. J., 1982, M.N.R.A.S., 199, 37.

Branch, D., and Nomoto, K. 1986, Astr. Ap., 164, L13.

Bromage, G. E., and Nandy, K. 1983, M.N.R.A.S., 204, 29 P.

Burbidge, E. M., Burbidge, G. R., Fowler, G. R., and Hoyle, F. 1957, Rev. Mod. Phys., 29, 547.

Burki, G. 1977, Astr. Ap., 57, 135.

Butcher, H. R. 1977, Ap. J., 216, 373.

Cameron, A. G. W. 1982a, Ap. Space Sci., 82, 123.

_ 1982b, in "Essays in Nuclear Astrophysics", ed. C. A. Barnes, D. D. Clayton, and D. N. Schraam (Cambridge: Cambridge University Press), p. 23. 
Castellani, V., Chieffi, A., Pulone, L., and Tornambè, A. 1985, Ap. J. (Letters), 294, L31.

Chièze, J. -P. 1987, Astr. Ap., 171, 225.

Desikachary, K. and Hearnshaw, J. B. 1982, M.N.R.A.S., 201, 707.

Donas, J. and Deharveng, J. M. 1984, Astr. Ap., 140, 325.

Dopita, M. A. 1985, Ap. J. (Letters), 295, L5.

Dopita, M. A. 1987a, in IAU Symposium 115, Star Forming Regions, ed M.Peimbert and J. Jugaku (Dordrecht: Reidel), p. 501. 1987b, Ap. J., (submitted). . 1987c, Ap. J., (submitted). . 1989, (preprint).

Dufour, R. D. 1984, in IAU Symposium 108, Structure and Evolution of the Magellanic Clouds, ed. S. van den Bergh and K. S. de Boer (Dordrecht: Reidel), p. 353. . 1986, Publ. Astr. Soc. Pac., 98, 1025.

Dufour, R. J., Schiffer, F. H., And Shields, G. A. 1984, in "The Future of Ultraviolet Astronomy Based on Six Years of IUE Research", (NASA Conf. Publ.), p. 111.

Dufour, R. J., Shields, G. A., and Talbot, R. J. Jr. 1982, Ap. J., 252, 461.

Evans, I. N., and Dopita, M. A. 1985, Ap. J. Suppl., 58, 125.

Filippenko, A. V., and Sargent, W. L. W. 1986, Astr. J., 91, 691.

Fitzpatrick, E. L., and Savage, B. D. 1984, Ap. J., 279, 578.

Franco, J. 1983, Ap. J., 264, 508.

Franco, J., and Cox, D. P. 1983, Ap. J., 273, 243.

Fransson, C., Cassatella, A., Gilmozzi, R., Kirshner, R. P., Panagia, N., Sonneborn, G., and Wamsteker, W. 1989, Ap. J., 336, 429.

Frantsman, J. L. 1988, Ap. Spac. Sci., 145, 287.

Frogel, J. A., and Blanco, V. M. 1983, Ap. J. (Letters), 274, L57. 
Gaskell, C. M., Cappellaro, E., Dinerstein, H. L., Garnett, D. R., Harkness, R. P., and Wheeler, J. C. 1986, Ap. J. (Letters), 306, L77.

Gilroy, K. K., Sneden, C., Pilachowski, C. A., and Cowan, J. J. 1988, Ap. J., 327, 298.

Gratton, R. G. 1982, Ap. J., 257, 640. 1989, Astr. Ap., 208, 171.

Greggio, L., and Tosi, M. 1986, Astr. Ap., 156, L1.

Güsten, R., and Mezger, P. G. 1983, Vistas in Astr., 26, 159.

Hawkins, M. R. S. and Brück, M. T. 1982, M.N.R.A.S., 198, 935. .1984, in IAU Symposium 108, Structure and Evolution of the Magellanic Clouds, ed. S. van den Bergh and K. S. de Boer (Dordrecht: Reidel), p. 353.

Humphreys, R. M., and McElroy, D. B. 1984, Ap. J., 284, 565.

Hunter, D. A., Gallagher, J. S., and Rautenkranz, D. 1982, Ap. J. Suppl., 49, 53.

Iben, I. Jr., Nomoto, K., Tornambè, A. Tutukov, A. 1987, Ap. J., 317, 717.

Iben, I. Jr., and Renzini, A. 1983, Ann. Rev. Astr. Ap., 21, 271.

Iben, I. Jr., and Tutukov, A. V. 1984, Ap. J. Suppl., 54, 335. . 1985, Ap. J. Suppl., 58, 661.

Israel, F. P. 1984, in IAU Symposium 108, Structure and Evolution of the Magellanic Clouds, ed. S. van den Bergh and K. S. de Boer (Dordrecht: Reidel), p. 319.

Kettner, K. U., Becker, H. W., Buchmann, L., Gorres, J., Krawinkel, H., Rolfs, C., Schmalbrock, P., Trautvetter, H. P., and Vlieks, A. 1982, Z. Phys., A308, 73.

Kirshner, R. P., 1988, in IAU Colloquium 108, Atmospheric Diagnostics of Stellar Evolution: Chemical Peculiarity, Mass Loss, and Explosion, ed. K. Nomoto (Berlin: Springer), p. 252.

Koorneef, J., and Code, A. D. 1981, Ap. J., 247, 860. 
Lambert, D. L. 1989, preprint.

Larson, R. B. 1981, M.N.R.A.S., 194, 809. . 1985, M.N.R.A.S., 214, 379. . 1986, M.N.R.A.S., 218, 409.

Lequeux, J. 1979, Astr. Ap., 71, 1. 1984, in IAU Symposium 108, Structure and Evolution of the Magellanic

Clouds, ed. S. van den Bergh and K. S. de Boer (Dordrecht: Reidel), p. 67.

Luck, R. E., Bond, H. E. 1985, Ap. J., 292, 559.

Luck, R. E., and Lambert, D. L. 1981, Ap. J., 245, 1018.

Lyubimkov, L. S., and Boyarchuk, A. A. 1982, Astrofizika, 18, 596.

Magain, P. 1989, Astr. Ap., 209, 211.

Malaney, R. A. 1987a, Ap.J., 321, 832. 1987b, Ap. Space Sci., 137, 251.

Matteucci, F. 1986, Ap. J. (Letters L, 305, L81.

Matteucci, F., and Chiosi, C. 1983, Astr. Ap., 123, 121.

Matteucci, F., and Greggio, L. 1986, Astr. Ap., 154, 279.

Matteucci, F., and Tornambé, A. 1985, Astr. Ap., 142, 13.

Matteucci, F., and Tosi, M. 1985, M.N.R.A.S., 217, 391.

Mathewson, D. S. and Ford, V. L. 1984, in IAU Symposium 108, Structure and Evolution of the Magellanic Clouds, ed. S. van den Bergh and K. S. de Boer (Dordrecht: Reidel), p. 125.

Nandy, K., Morgan, D. H., and Houziaux, L. 1984, M.N.R.A.S., 211, 895.

Nomoto, K. 1984, in Stellar Nucleosynthesis, ed. C. Chiosi, and A. Renzini, (Dordrecht: Reidel), p. 239.

Nomoto, K., Thielemann, F. K., Yokoi, Y. 1984, Ap. J., 286, 644.

Norman, C., and Silk, J. 1980, Ap. J., 238, 158.

Olaffsson, K., Bergrall, N., and Ekman, A. 1984, Astr. Ap., 137, 327. 
Pagel, B. E. J., 1988, in Evolutionary Phenomena in Galaxies, ed. J. Beckman, and B. E. J. Pagel (Cambridge: Cambridge University Press), (in press).

Pagel, B. E. J. 1985, in ESO Workshop No. 21 on Production and Distribution of $C, N, O$ Elements, ed. I. J. Danziger, F. Matteucci, and Kjär, K. (ESO, Garching, 1985), p. 155.

Pagel, B. E. J., Edmunds, M. G. 1981, Ann. Rev. Astr. Ap., 19, 77.

Pagel, B. E. J., Edmunds, M. G., Fosbury, R. A. E., and Webster, B. L. 1978, M.N.R.A.S., 184, 569.

Reitermann, A., Stahl, O., Wolf, B., Baschek, B., and Scholz, M. 1988, talk given at the IAU General Assembly, Baltimore.

Reike, G. H., Catri, R. M., Black, J. H., Kailey, W. F., Mc Alary, C. W., Lebofsky, M. J., and Elston, R. 1980, Ap. J., 290, 116.

Reike, G. H., Lebofsky, M. J., Thomson, R. I., Low, F. J., and Tokunaga, A. T. 1985, Ap. J., 238, 24.

Renzini, A., Bernazzani, M., Buonanno, R., Corsi, C. E. 1985, Ap. J. (Letters), 294, L7.

Renzini, A., and Voli, M. 1981, Astr. Ap., 94, 175.

Reynolds, S. E., Hearnshaw, J. B., and Cottrell, P. L. 1988, M.N.R.A.S., 235, 1423.

Russell,.S. C., and Bessell, M. S. 1989, Ap. J. Suppl., (in press).

Russell, S. C., and Dopita, M. A. 1989, Ap. J., (submitted).

Scalo, J. M. 1986, Fund. Cosmic Phys., 11, 1.

Scoville, N. Z., and Good, J. C. 1987, in Star Formation in Galaxies, ed. C. J. Lonsdale Persson (Pasadena: NASA Conference Publishers), p. 3.

Scoville, N. Z., Sanders, D. B. and Clemens, D. P. 1986, Ap. J. (Letters), 310, L77.

Seeger, P. A., Fowler, W. A., and Clayton, D. D., 1965, Ap. J. Suppl., 97, 121.

Serrano, A., Peimbert, M. 1983, Rev. Mex. Astr. Astrofis., 8, 117. 
Shaver, P. A., McGee, R. X., Newton, L. M., Danks, A. C., and Pottasch, S. R. 1983, M.N.R.A.S., 204, 53.

Sneden, C. 1985, in ESO Workshop No. 21 on Production and Distribution of $C, N, O$ Elements, ed. I. J. Danziger, F. Matteucci, and K. Kjär (ESO, Garching, 1985), p. 1.

Spite, M., Barbuy, B., Spite, F. 1989, preprint.

Spite, M., Cayrel, R., Françcois, P., Richtler, T., and Spite, F. 1986, Astr. Ap., $168,197$.

Spite, M., and Spite, F. 1978, Astr. Ap., 67, 23.

Spite, F., Spite, M., and François, P. 1989, Astr. Ap., 210, 25.

Stryker, L. L. 1981, PhD. Thesis, Yale University.

Stryker, L. L., and Butcher, H. R. 1981, in Astrophysical Parameters for Globular Clusters, IAU Colloq. No. 68, ed. A.G.D. Philip, and D.S. Hayes (Schenectady: L.Davis Press), p. 255.

Thielemann, F. K., Nomoto, K., and Yokoi, K. 1986, Astr. Ap., 158, 17.

Thielemann, F. K., Arnett, D. W. 1985, Ap. J., 295, 604.

Tornambè, A., and Matteucci, F. 1987, Ap. J. (LettersL, 318, L25.

Tosi, M. 1988, Astr. Ap., 197, 33.

Tosi, M., and Diaz, A. 1985, M.N.R.A.S., 217, 571.

Truran, J. W. 1981, Astr. Ap., 97, 391.

van den Bergh, S., McClure, R. D., and Evans, R. 1987, Ap. J., 323, 44.

Wheeler, J. C., Sneden, C., and Truran, J. W. Jr. 1989, Ann. Rev. Astr. Ap., 27, (in press).

Whelan, J. C., and Iben, I. Jr. 1973, Ap. J., 186, 1007.

Woosley, S. E., and Weaver, T. A. 1982, in Essays on Nuclear Astrophysics, ed. C. A. Barnes, D. D. Clayton, and Schramm, (Cambridge University Press), p. 377. 1986, Ann. Rev. Astr. Ap., 24, 205. 
Wyse, R. F. G., and Silk, J. 1989, Ap. J., 339, 700. 